Australia 1988 $2500-10 \mathrm{NOV}$ 


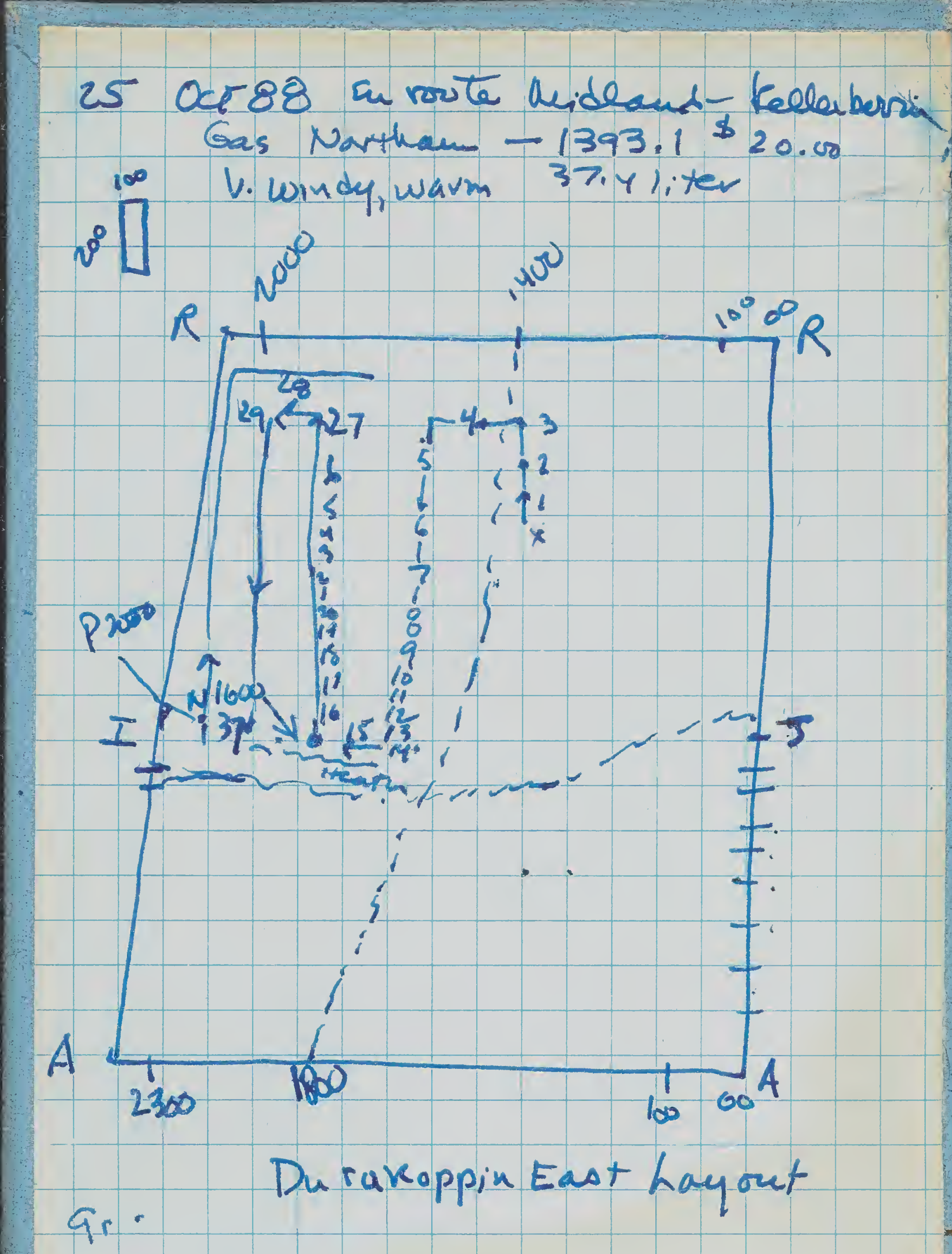

qr.

$$
\begin{aligned}
& 26 \text { oct Keelenkerrin area } \\
& \text { Durakmpin Eost - kqin cemes } \\
& \text { at osius ceen, cool, calm. }
\end{aligned}
$$

\begin{tabular}{|c|c|c|c|c|c|}
\hline 3 & & & & \multicolumn{2}{|c|}{$\therefore$ Celoge of arode. -0} \\
\hline & 2 & & & 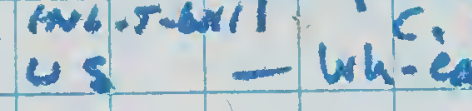 & riHE-ysina \\
\hline & $n$ & " & 1 & - 0 & Coonwh \\
\hline 6 & " & i & . & -0 & \\
\hline
\end{tabular}

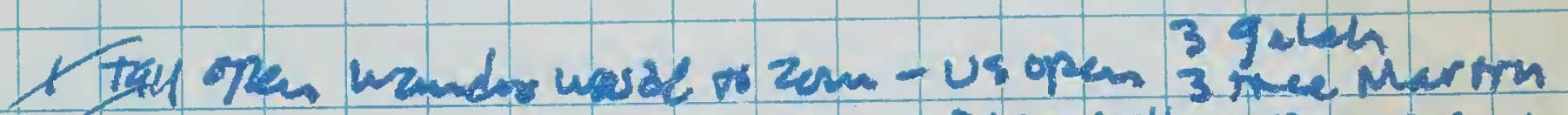

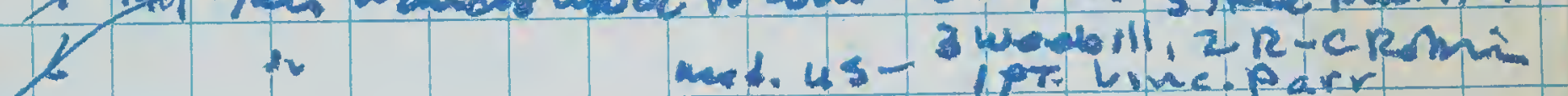

7 farly rollwandoo (cur -12-18m)-mod dence us $\sim 3$ chr

8 opar York quen/dam wod CHT $\sim 2 \mathrm{~mm}$, mad as - 0

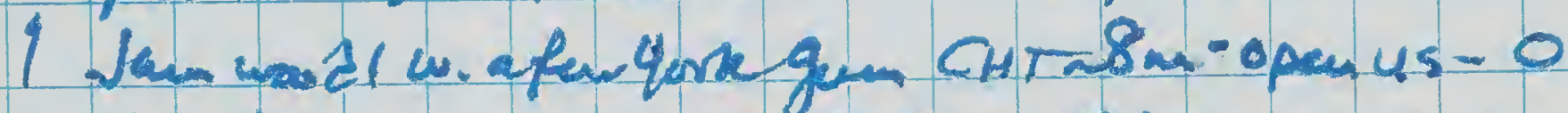

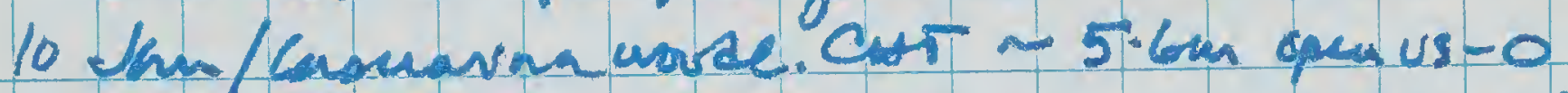

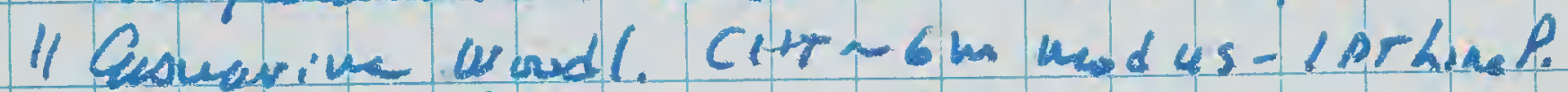

12 Cammona/then uasdl - 5-7an - 0

13 Gsuarma wasde.- " " mo

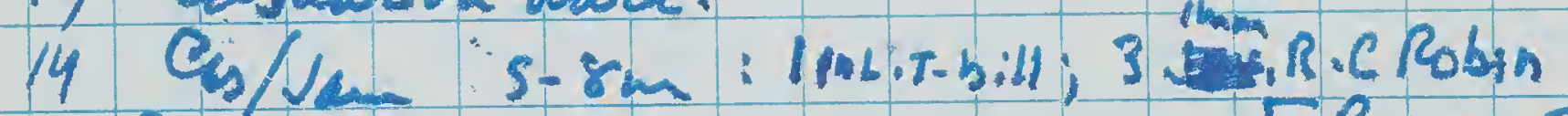

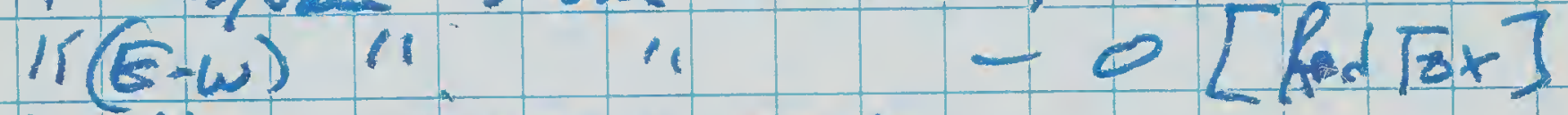
16 N-160u-stawt nark any'ros line 2 Cn/Vam unde. S-6m-modus - 0

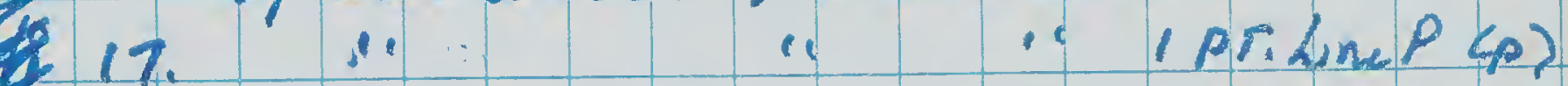
18 open Cas/Jam uonds 5.6 an - 0

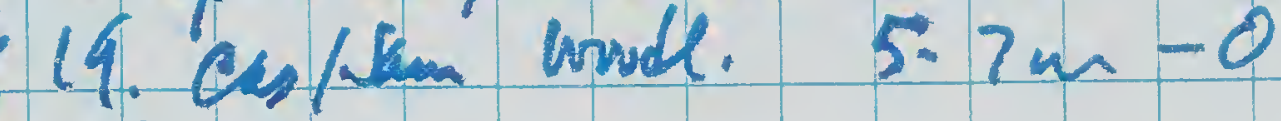

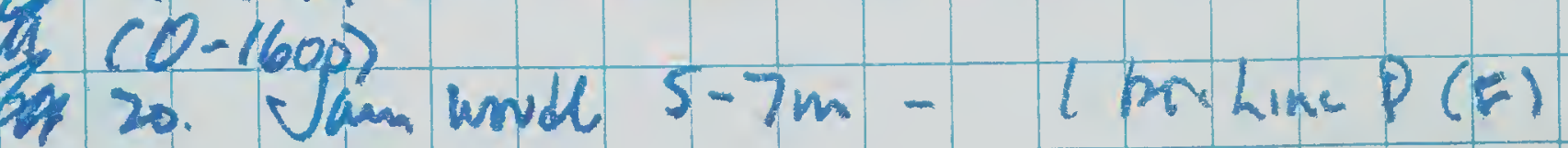
21 1. u u 20 22 Yurk (Wando Wooll. ISina imod US - O 
Uot-cont.d

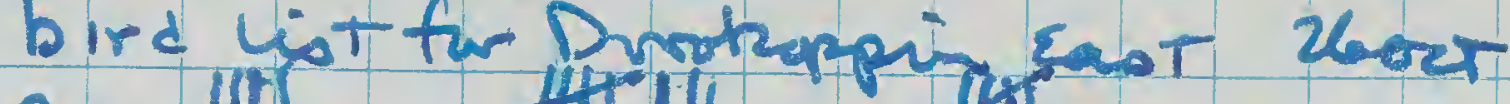

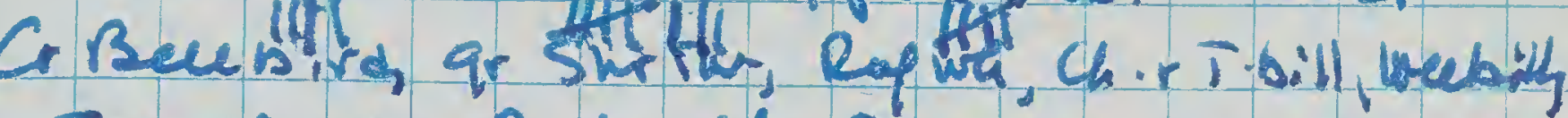
Tree Martin Pr.hicolin Pingrede, galeh, Sor Pandaloce, Wh-eurt HE, In) T-bin, RCRobin, Ausp. Ruven, sh BrCuckes Y-T Miner, Y-KT.bill ;C.Brongain, Brite, Austrillape 5. Sermb-Robm, Crented Pigeon, sp.CAHE, Sony HE, Wh-br Babbler, MulqaParrot

cusas 5 cotion 38

S. Surab Robin

Peeep - Chr-Chr-Chr-chr rapid chattar

\section{mosty sandy of litter} on groner tevee

23 apen banders avo dl - 15 m-Fily lune us - 0 $(p-1600)$

24. " 4. Jimfar 4s-mod shrabs-0

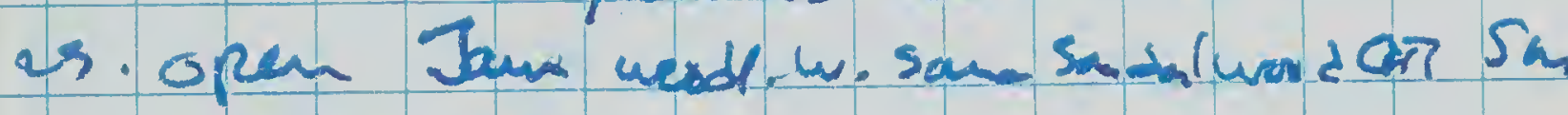
tpan us

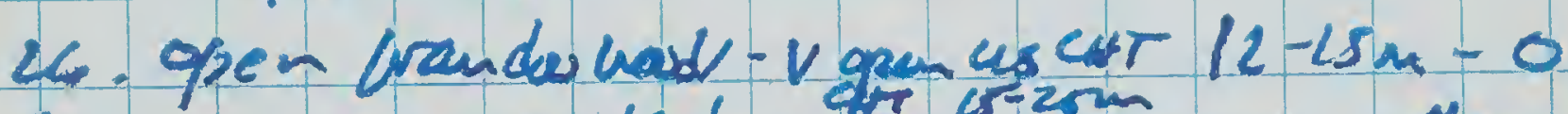

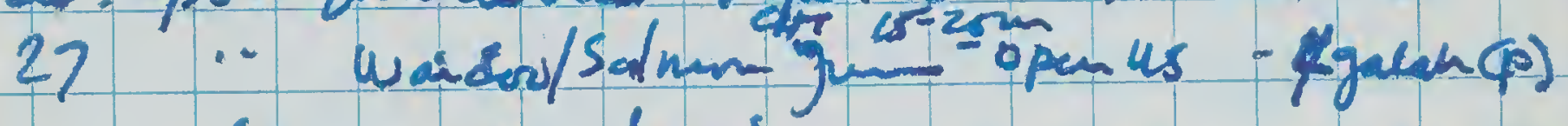
(turn wat here)

C.Browzeng $\sim 5$ Y-RT-

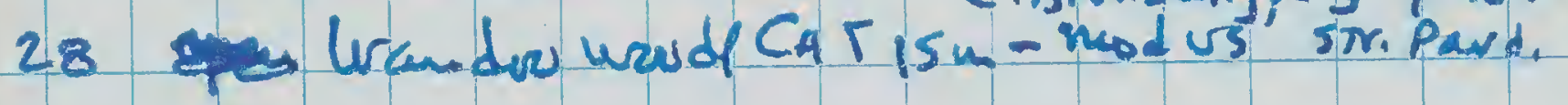

$$
\text { Q }-1700 \text { - turns. }
$$

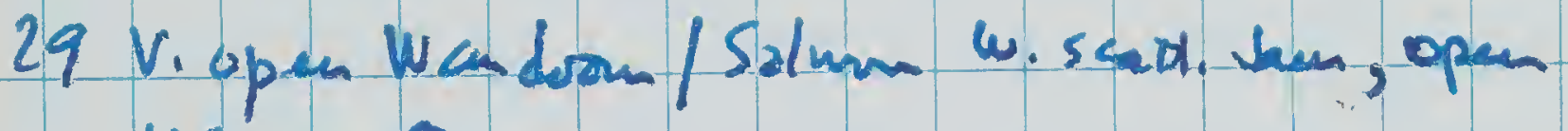
us. - 0

$30 \ldots$

$$
\text { - } 0
$$

31. Mod. wan do woodl - 1072m - 1/2 apm,

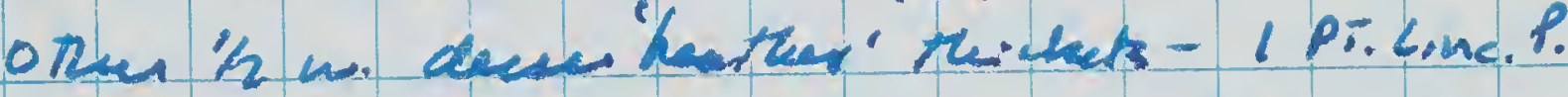

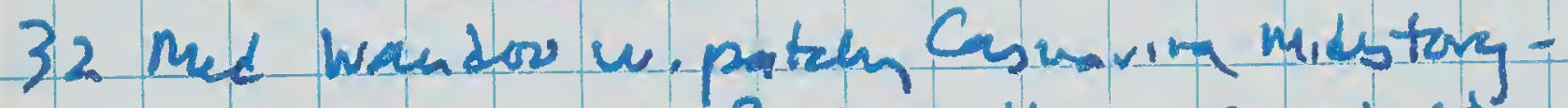
opan u.s- 3 Weahills, galah $(p)$

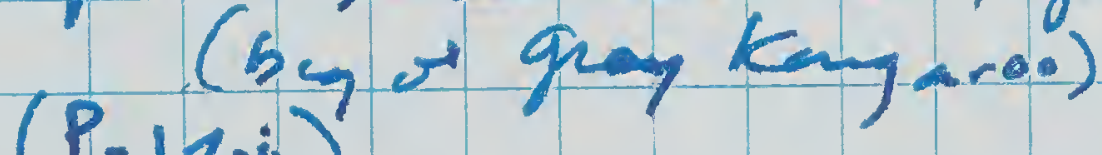
$\left(P-110^{\circ}\right)$

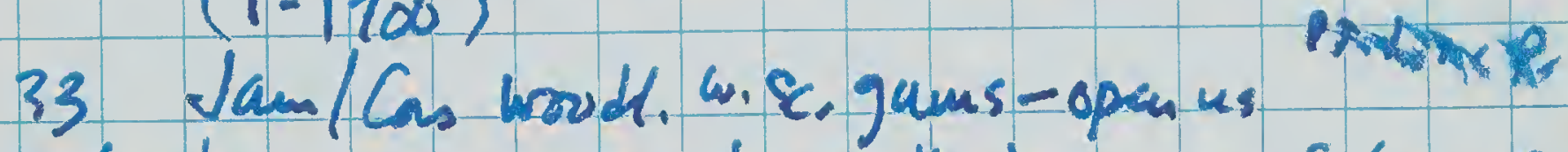
34. Jan / Cas W. Save heath thichats cut S $6 m-0$ 35. open York Cati (on) n. dune meluearen, oTher shrobs - 2 Brite,

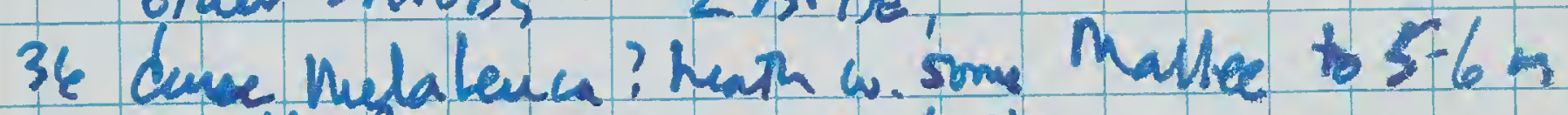
4 wh-ened HE, 2SP-Ch HE

Muple Claves is blom is Mulabeurear pentragona 
37. dewe thel (?) hath 4. Scutud kalke clumpotisn 2 wa - and $4 \varepsilon$

(moss tie rean to ke in inm. plunge)

(cutN-west here) M.cardiophylk-whele Melaleuca pentagona-punple

38. V-deure thel un seatud

wacles, Acuers to $4 \mathrm{~m}$

15. SanbRobir, BrHE

Cal: high pikhed "eqt-a-beet!"

wo a lud, hareh scortiy Ch.r.r.r!

eceet-chrrr - ch-ch-ch-ch-ch-ch highthin kawh viapidchofer

(unde mune gord reancip)

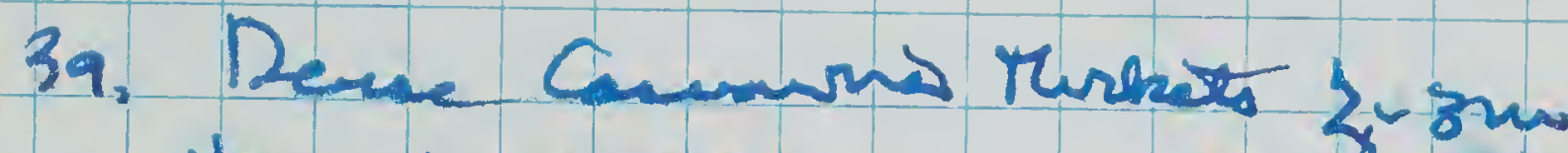
tas. impentrate gr shrthe

Tho at Dimis Kangaros liñe

4 Cut Nort heve

Co Dewe Cas. Thichots (sma terweij) 2-3x

4\%. operyork gum h. dene os of knta

ces, Acáind et. CitT $216 \mathrm{~m}$ -

(P.2000 hure)

42 Vam evarde CNAD $(3-5 a)$ firlyopan us

43 " $"$ "

(Grshrtan and Rey wh $100 \mathrm{~m}$ E)

44 . $"$ a. Mad us. 2poting

is. Jam/las unde 3-5m tirly open us -o La tarn E. here at Q-2000 (kear nend)

46. open Jam unsd. Zuod. us ChT 3.4 m - 0

$47 . " \quad " \quad \varphi 1900$

48 " " "

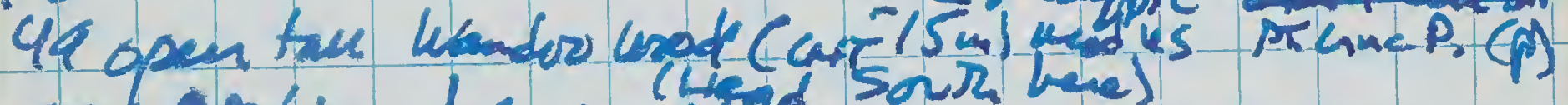

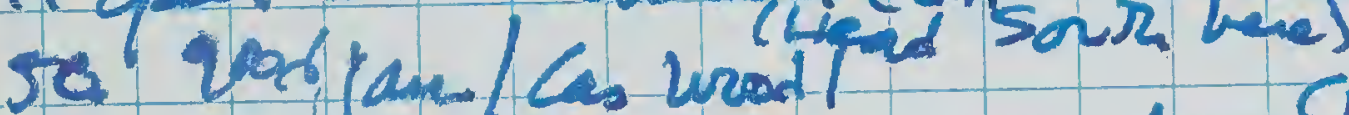

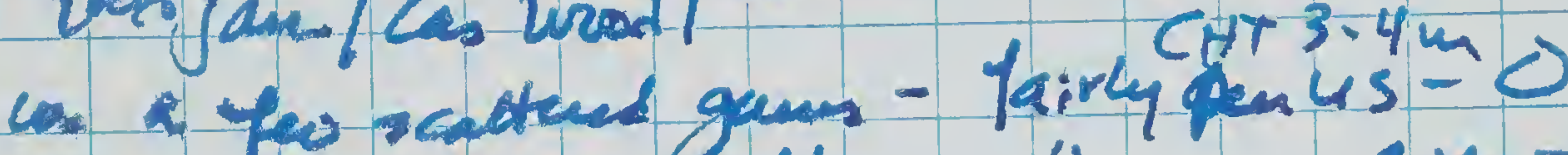

s) Ian 16. worde $\$ 4 m-"$ ". 2YkTbill

52 N $\cdots \cdots \cdots-0$

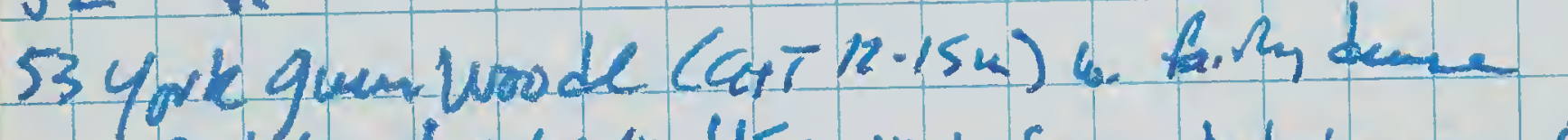

potidy shirobs in 45. iny, Sem helabura - 0

sy Mallee (CHr8) 11 - Rufwirith

Sigi (Some Terv, as on 2200688) BrHE Sinqite (at East here)

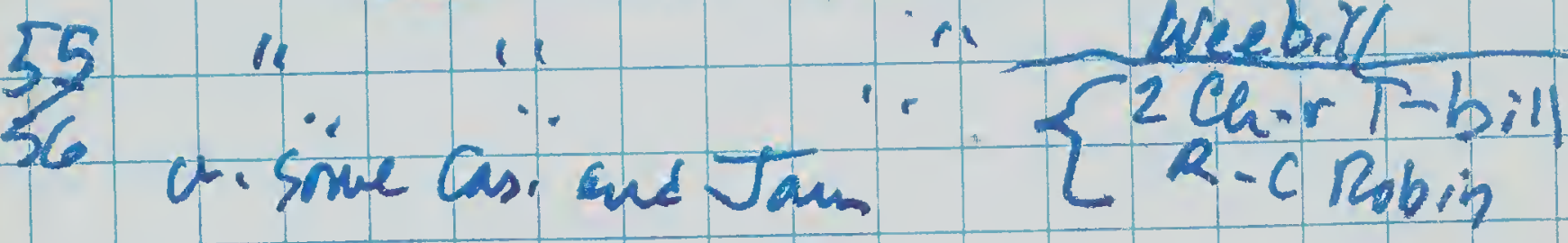

57 open Yorkqun und ( Cart 6-8 m) W.

Lau wid-stary hod sunb den is $>0$ 
NS Kaysaroo livelune (1600?) beat $N$.

58. open weredor ande. CAT $\sim 12-15 \mathrm{im} w$.

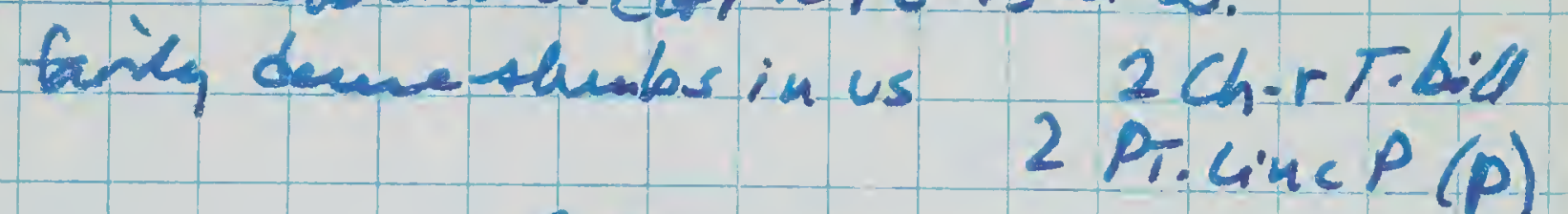

"

Sq (P1600 kacfuren Thing this seg.)

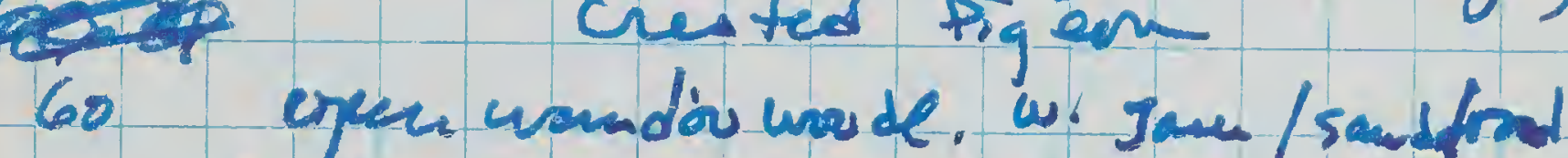

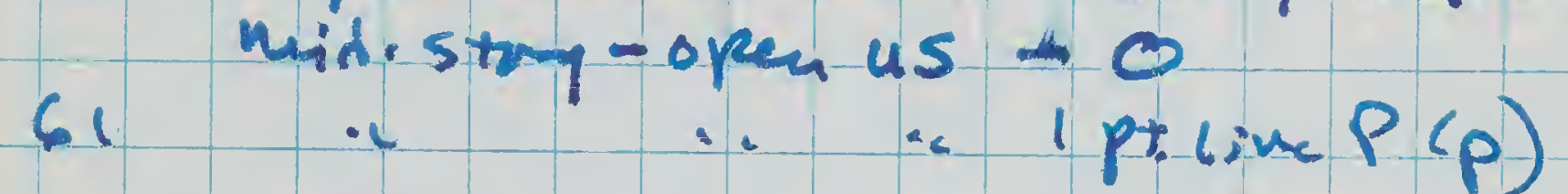
(Skip nest bit and po X. suto bent, Then hand tast I

62 doner dvere kurigan - mel. qraitles setqu, Geummx, Acariz, ete Outra $2 m$ 63 2 Brite

$64 \quad 11 \quad * \quad * 2$ bublew $65 \%$ r -0 Pr+te (access us nack hay way taryz

$6 \varepsilon$.

$67 . \quad \cdots \quad \cdots \quad-0$ 68 " $\cdots \cdots \cdots+\infty \quad \cdots$ 49 $\cdots \cdots \cdots \cdots$ 70. $\cdots \ldots \ldots$ SINE HE (yeccor qrew:lea in bom -abo (q. pale orange one)

76. le il i is 0 72 [Scastuad jaw to $4 \mathrm{~m}$ ] 73. (u. Suttend Jan) $\cdots-0$

74

75.

76 .

$$
\begin{aligned}
& \cdots \quad \cdots+\infty-\infty \\
& \begin{array}{ccccc}
-\cdots & - & \cdots & -0 \\
\hline & - & \cdots
\end{array}
\end{aligned}
$$

3 qren komgares - one u. red tay on collar, which bad or maskes onit, / think

77. (st ky - detto; zurhy deune caswan Twickets Brits

78 dever las. Thichets z-3u high - O lead S. have stutat $0-1000$

79. Geuse thichets of lor 2 spp of shmbs CN $1.5-2.5 \mathrm{~m}$ - 
8/. dense shues Thichate 1.5-2.5 k high w.w.dely seatend Acacia to $4 \mathrm{~m}$ I BrHE

R Jau 'arbour' CHT 3-5m saoterad clumps of shrobs in us- nussthy vpenus 83 Jan (arkh' $4-6 \mathrm{~m}$ CHT. ORen

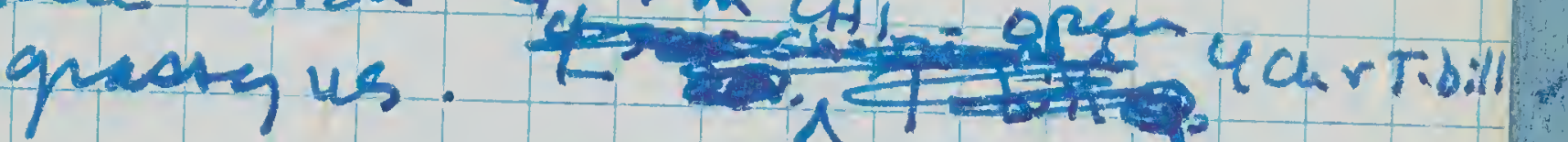

$84 . \quad \cdots \quad \cdots \quad$ (Y PT.Lirc.P. (P)

ares whisw tanged $\left\{2\right.$ Mulga $P_{0}(p)-P_{q}$

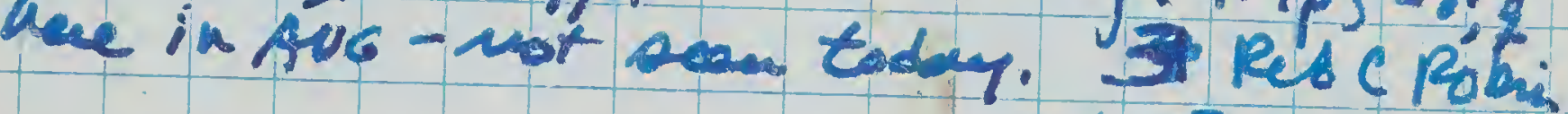

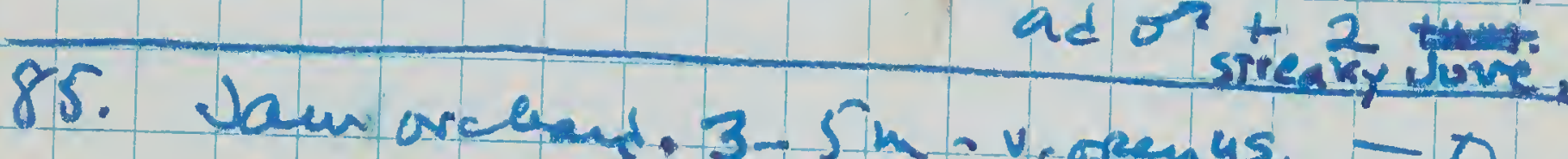

8

- cewscutial windar feedic o Elogant panst-coutlip

m-1000 hue

8 ? Waubso Woodland w. And katumbs ince feoven Astrolem fsecread in us

CRul whittar $12-15 \mathrm{~m}$. Sim. Rigeon 1001 in SE; aurther to ku. Hors/red's Broug - Cuckoosyy

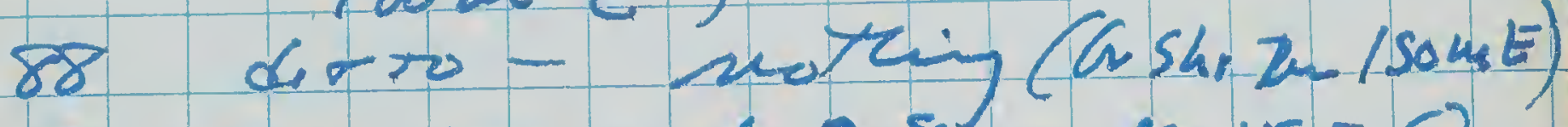
Fi ppen tam arch 3-sin - open us - 0 90. open Wandeow (CHT/2-15us $w$

unidaty of sam - fareqopen us - 0

91. « $2-1000 \quad-0$

q2. ox " " Mrddmesus - 0

Thi is ur. aub of ussoll at edge. of dense scunt - tame. hare

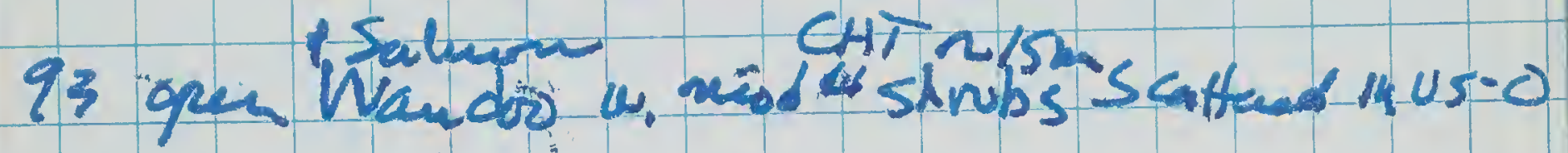

head N. $100 \mathrm{~m}$ E. of last line

94.

2- 3 uncels:ll

95.

Jalah $(p)$

96...$\quad \cdots \quad \cdots \quad$ (quan us) ad R.C Robin

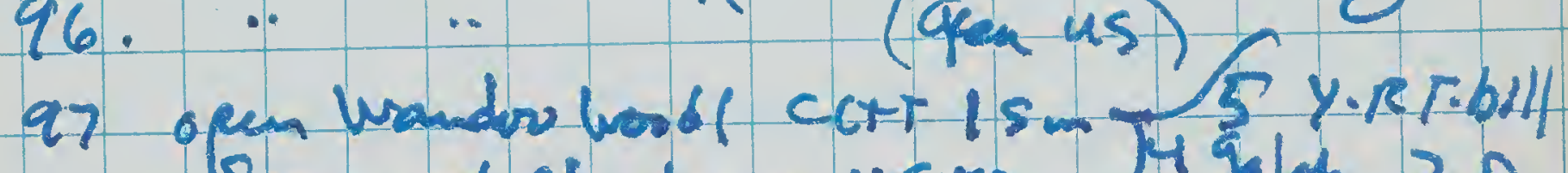

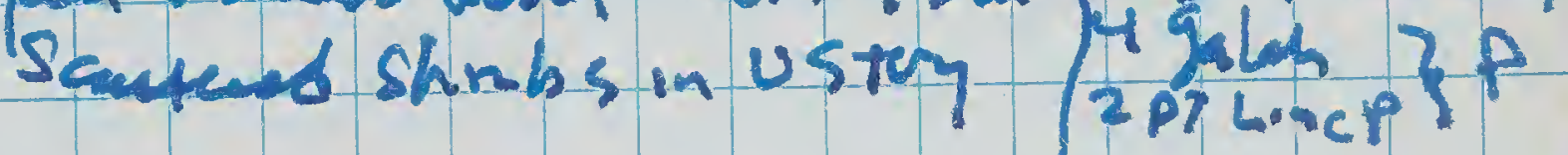

28 " "Jura. RC Robin qr shr turush

29 i. 


\section{Lent}

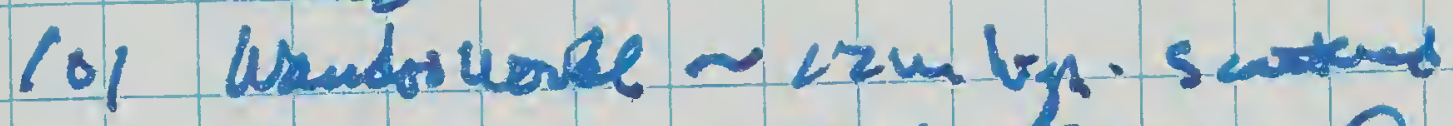
shmbomles - lotutsoplimp 0 CRee, whrten sing $100 \mathrm{~m}$ NW)

Mrent of waskenal - unt east 100 in to nest Roo line

loz a E.w. . . - 0 103 - an lor cire-ased 5. 18 Wands/Salum geum wa satat sumbs in V. opea Ustery

Clobut Prits sigin w $100-200 \mathrm{~m}$ aurey in malle. hearts

104.

los.

(M"sos hen

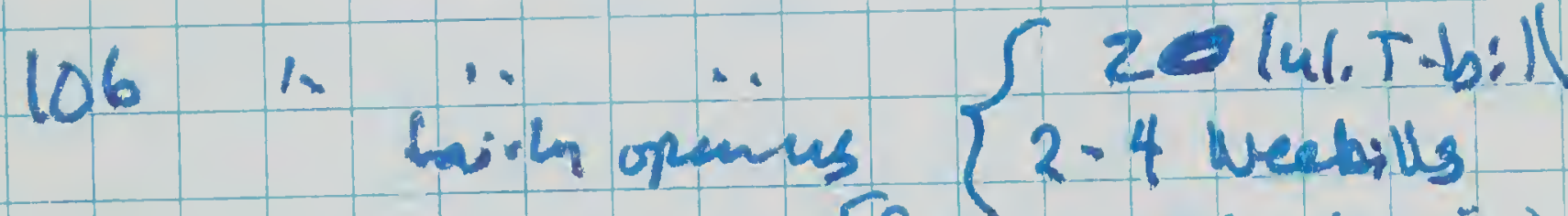

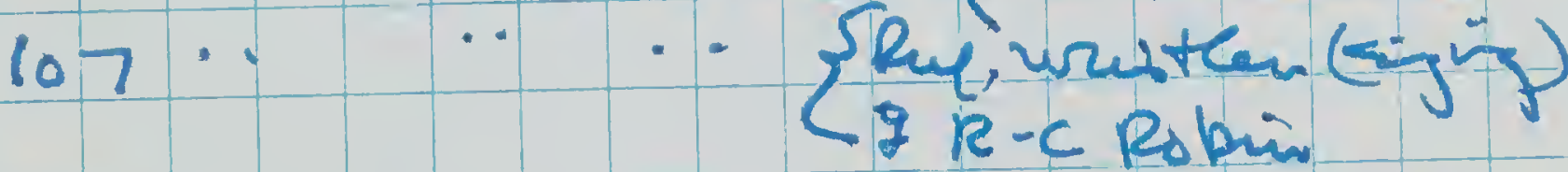
$\log \cdots \ldots-2$ galah $(p)$ loq " $" \because-0$ (L800 kee)

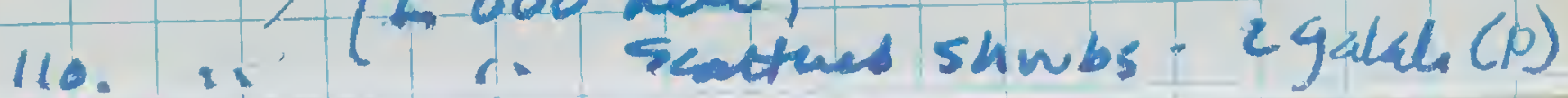

411. openterents wasd. Wo mid-sm 1 tom mi a scat. shinhs - 4 ch-ret.bill, sr. Aurd.

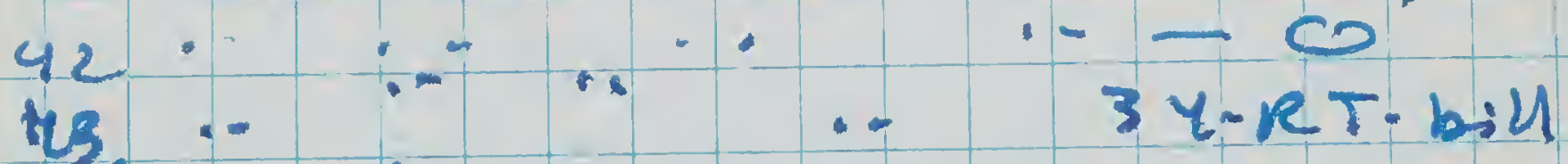
tr. - K 800 here-ns. edqur waste, sotumurest towand kest' Roo line.

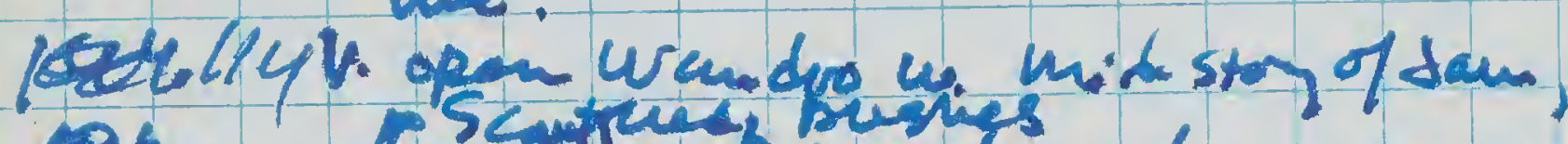

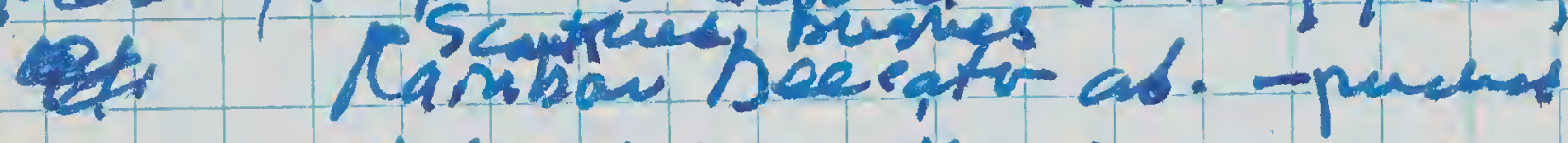
at ip 1 dead Voen / open area

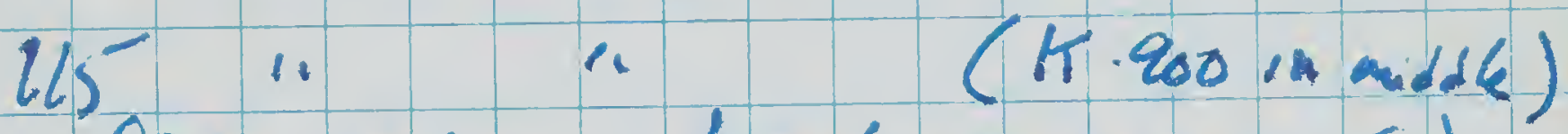
(acan ebye of by hark aven to 5.$)$

116 a " .. duve ReRgb:

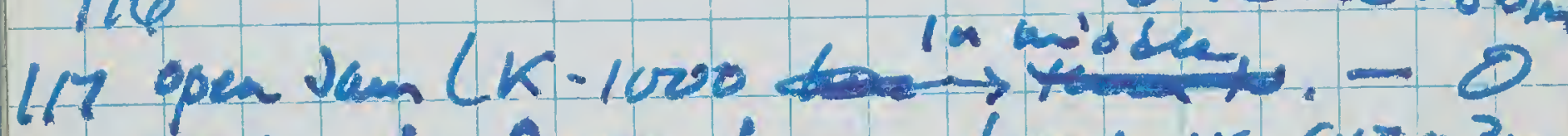

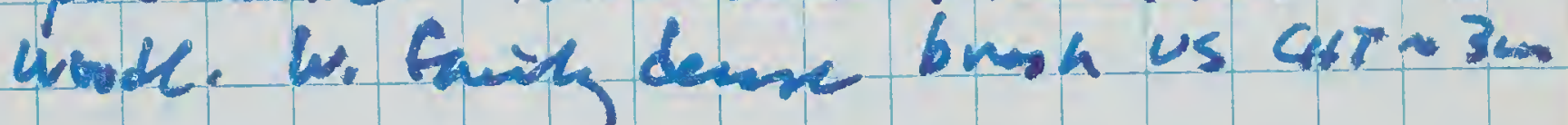

l18 open hath atr 1.5 in scatral Sandelumed.

c19." "l 3ch.reT.bill (K1100 midway hue)

$12 \ldots \ldots \ldots$

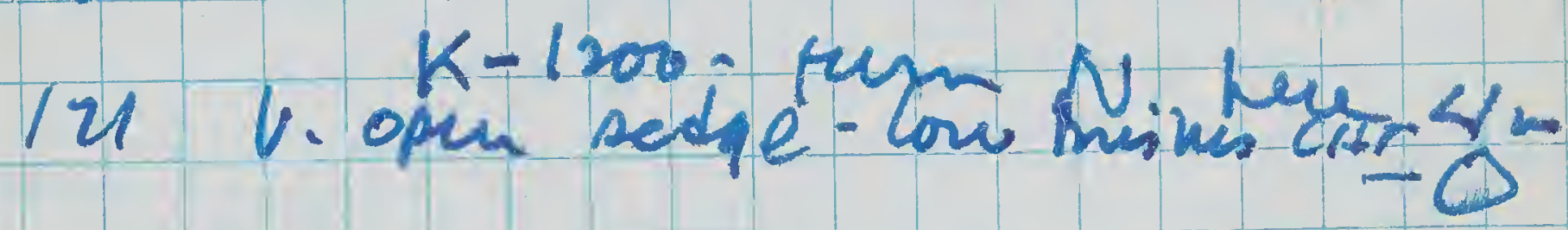
122. heteror, Kurorm cot 2-2,5m -0 
123. Mallee/ buth CNT $35.5 \mathrm{~m}$ Bitte (24. vipren Uzudoo u bory dune

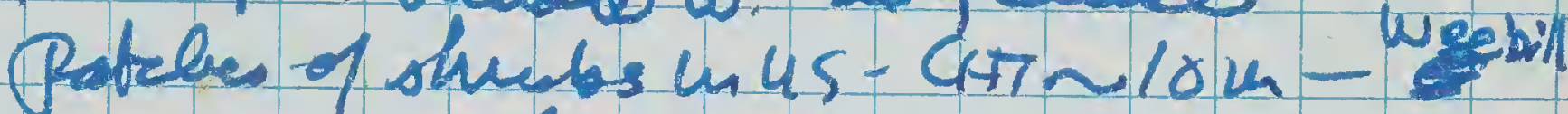
L-1200 hue

125. Open Jain/gum orch, w. taile

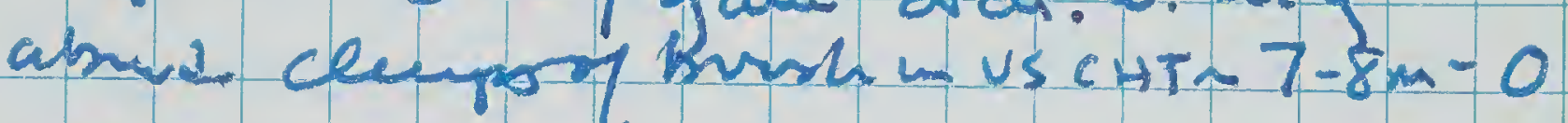
126 Nallee/Reath $C_{H}$ T $3-4 \mathrm{~m}$ ishribs fain doure - 0

127 heterg. kavongu-fairy dure arr 2-25n

128 oreu wrindor word. (Cur /2-15u)

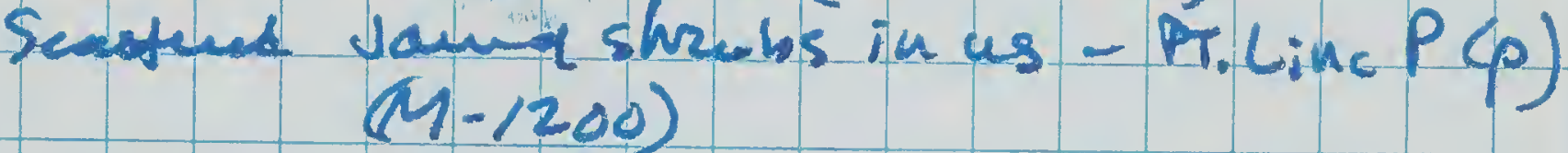

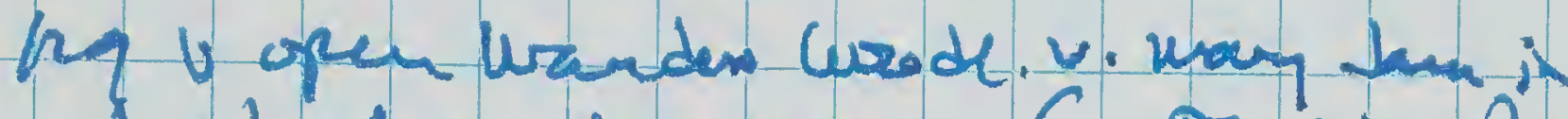

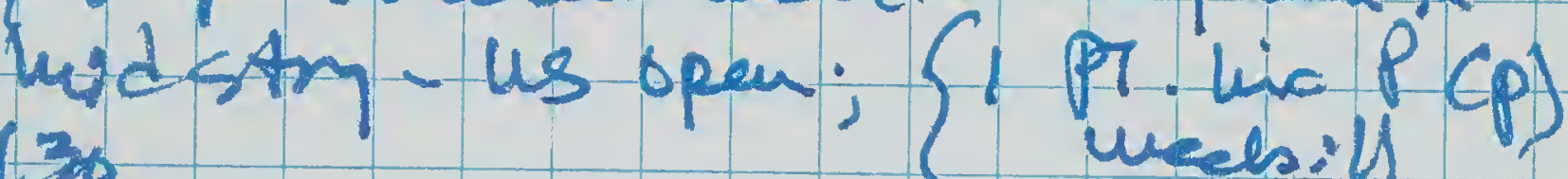
130

1.1. $-\infty$

131 bon arfard' Cat $4-6 \mathrm{~m}$ openus Y PT. Lin P. CG-tel

132 den haudor worde. W. Jan i Sandgluras Mid $\Rightarrow$ - Md shiwhs in us - 0 (N-1200kenes

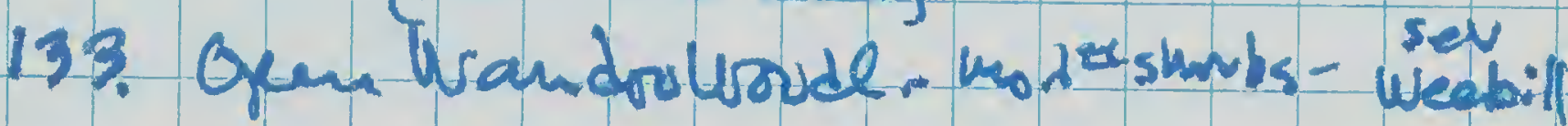

$134 \%-C$

135 open handwa. Giry dene shrib us - 0

$136 \ldots \ldots \ldots$ turn w. baels to Car $(37 \ldots-0$ head NW olny trail

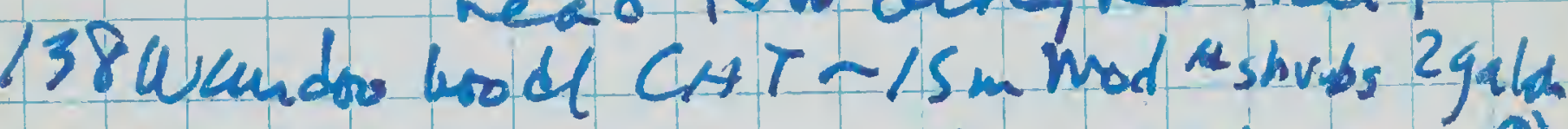
139 i. 14 weebill 9) 140 i $\quad \cdots-0$ $141 \ldots \ldots$. -6 142 " " " 2 YRT-bill

end at $16: 45$ back a car 
13islirs - Edge of pune undi s. Arm canss trach av E. S.Le

143. opene dam / Cas. 4. deme ceungs of 3 .3m brosh and frows-w. Wrytail, 3BF wasdsu. lel Jam orch - sestend beatur in us CNT 3.5m-0 145. 1 . $\quad \cdots \quad$ - 0 (Ay whitar syjzllogm W.) $\leftarrow$ ) sum

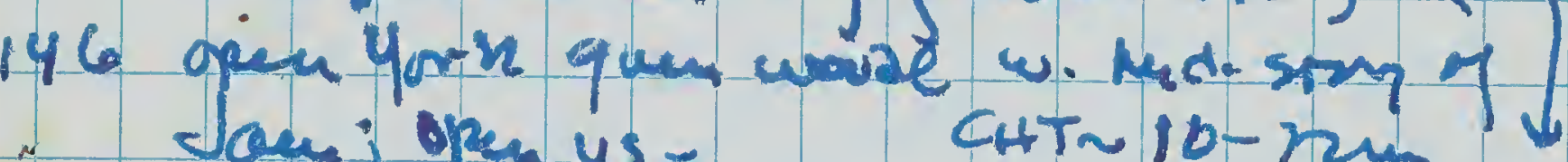

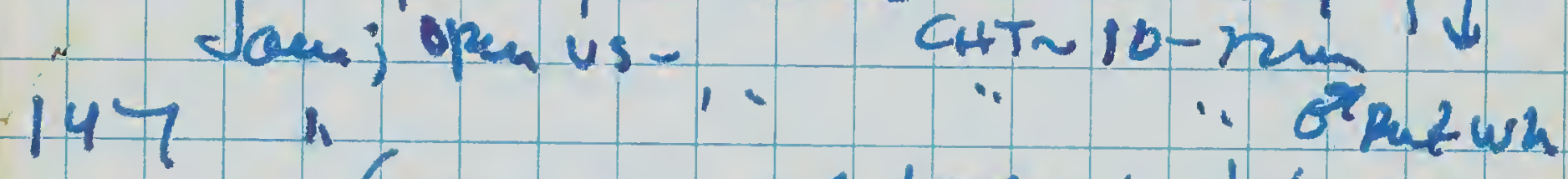
(tem 7 wh/Bleve in dily, twis)

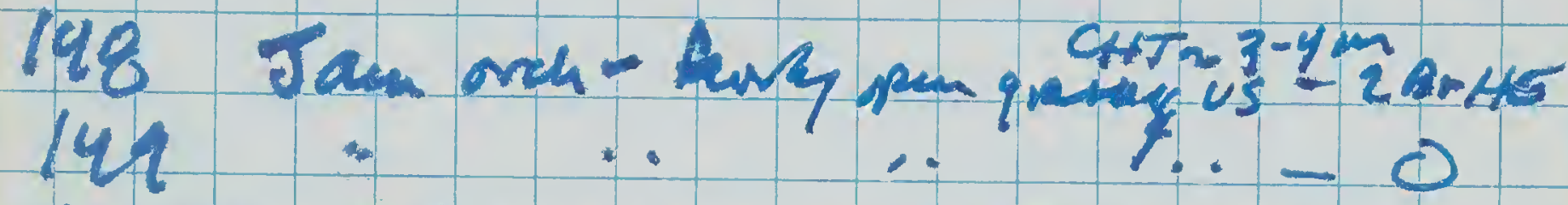
bo open saluom quen an. mod busthes

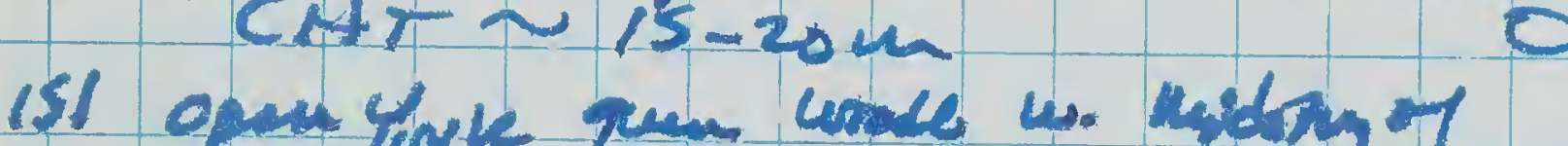

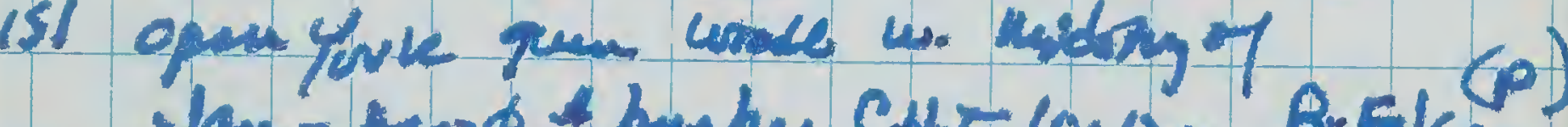

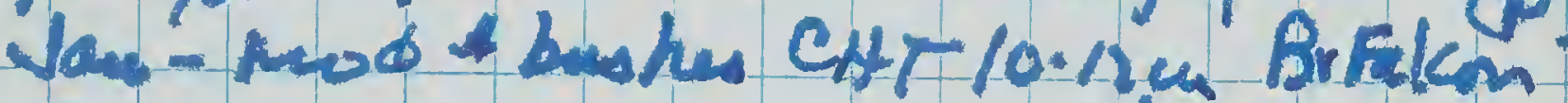
$4=21$. Weabrl $152 \cdots \quad \cdots \quad-0$

153 apen York wodd. men men Jach in midston CHT $25 \mathrm{~m}$ BLF wadsw. (Rol. Whither + Bellbord srowi $100 \mathrm{~m} W$ )

\section{4}

iss qrod York hood, wi Jain 2 Yke T-bill mundstion- $\cos 216 \mathrm{~m}$ Brom He

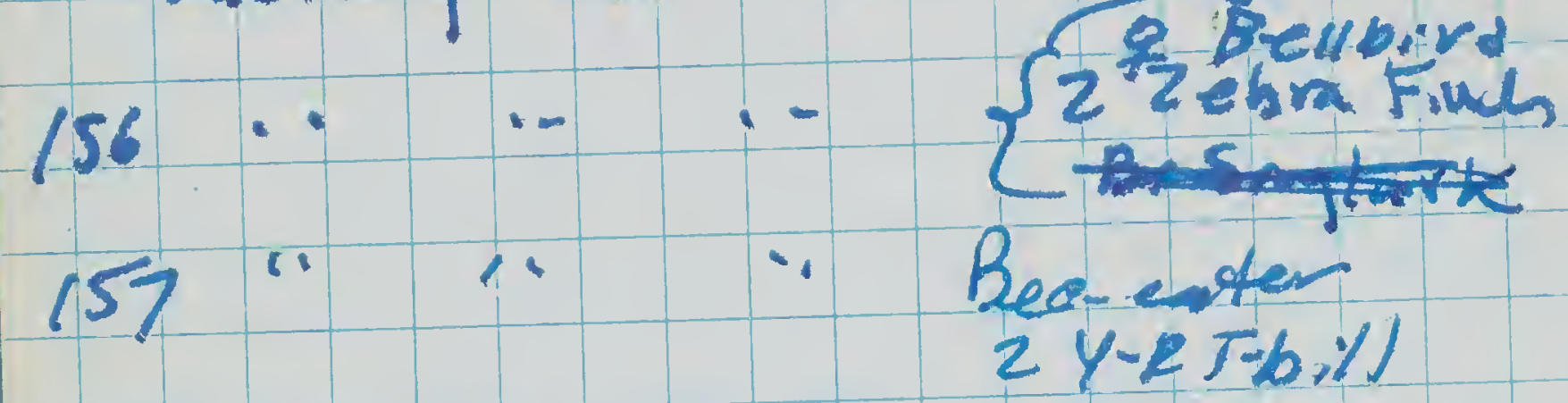

158 Yowk/Wando ond w. Los 7 tum (a us - xoyfaed shmbs 2 alah ( $p$ ) IsI .. .. .. meemarti

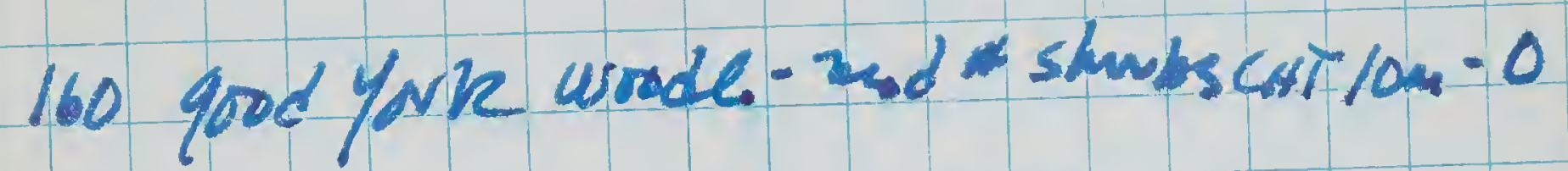
Ulal" "lotsy wilbats in us - 0

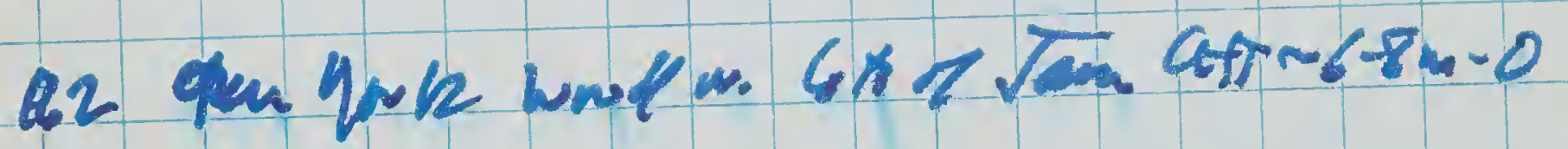
(6) 3 Jan/haken orh-opmus cot $\sim 3 \mathrm{~m}$

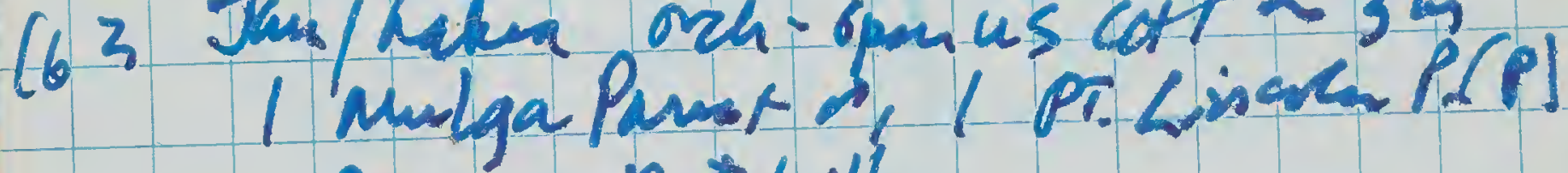
sw Y.R T-bill -0 
168. Jaufltakea / orkes parny as - 0 109 V. peen Tork/usurdoo w, mech Lamin midoty mod 4 shribs CHT $08 \mathrm{~m}$ - 0 110 bum/Ascha/ Sanda woodoch. opurgasan us CHT $\sim 3-5$ h -0

171

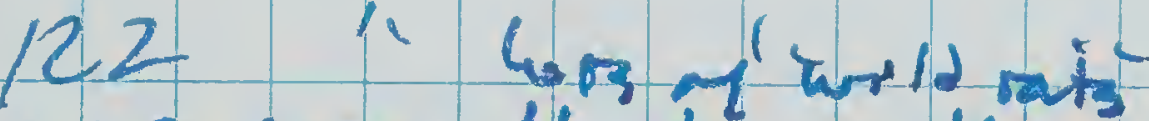

$173 \mathrm{rgpen}$ Yrik wrode w. Jam war-sty.

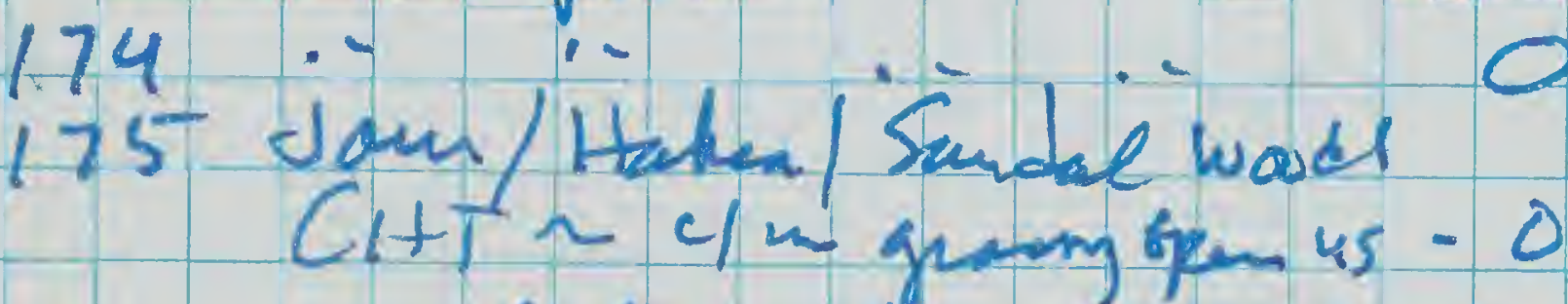
WTERI DR SZS

176. Yovr Woode, w. Jam midst. oporids CAT $\sim 12 \mathrm{2m}$ 3 yF Miver

177 claw orch gram us atia 35 in (a fru scottred Yark qum) - 0

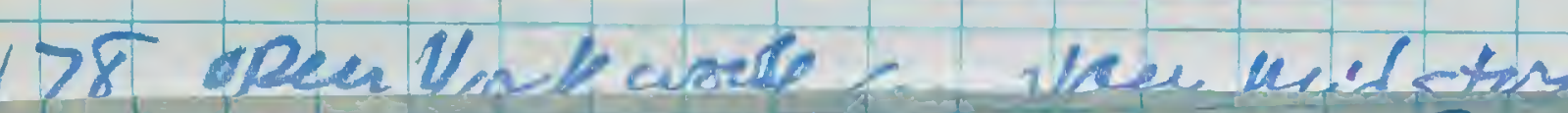

179 … $\quad$ Y Y Fnliner 180

I81 opulowkwoodl w vam/Ataken/medr bodft beeshes bis us

182 . $\quad \ldots \quad \ldots \quad 0$

183 ic isoúns 0

184 " 1 opeinis 0

18S..... open us 0

186 o " " " a 2 balaticp)

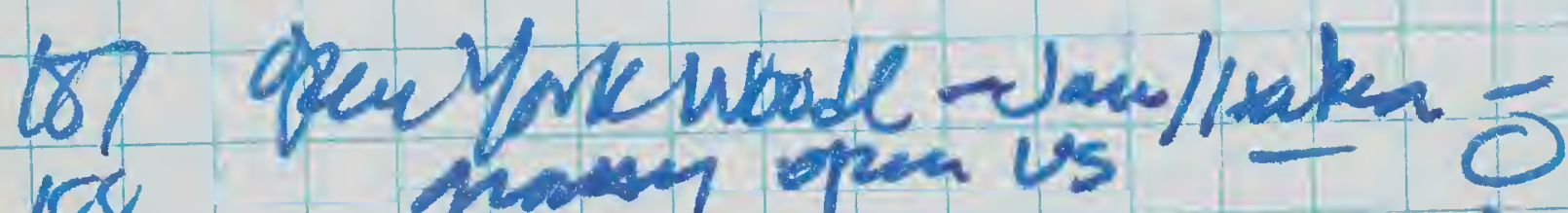
188 nasy ". 2Pr.hacP.

$\begin{array}{llll}189 & \ldots & \cdots & 0 \\ 190 \ldots, \ldots, & \ldots\end{array}$

lar $\cdots \cdots \cdots$

$192 \cdots \quad \cdots \quad \cdots$ RTiLind $(p)$

las wandooliond. Modet shnigrs 12-1ga lay .i ... .. . IPiLine (a) 11 " "2yTmin

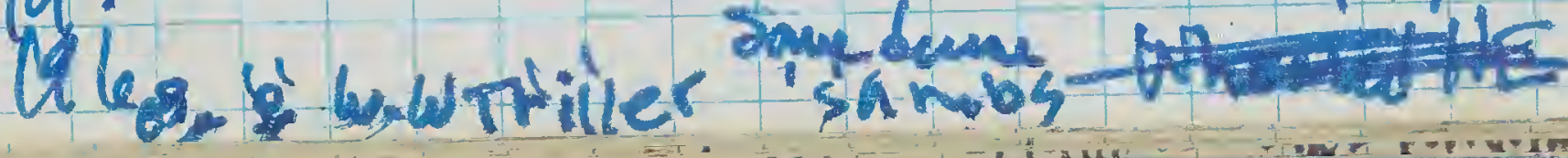


197 opeuqork wrode w Jam) Hatrea kud s. nod kusans un us. Pallid Cockero (cheses hy trillers), BKf Woudsurallow

148 dove R-c Bobin

$$
199 \cdot
$$

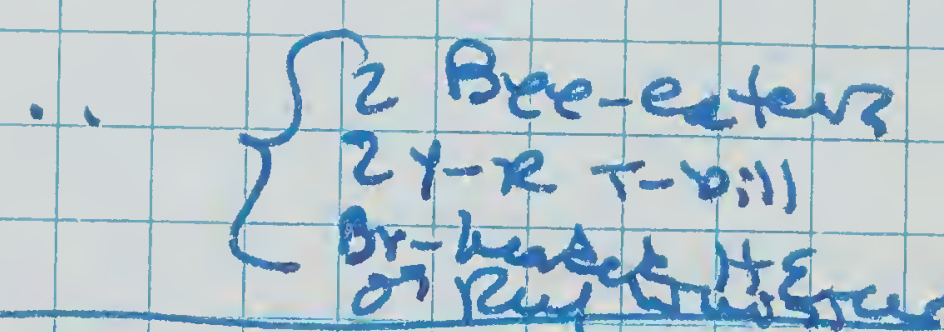

BR Ru/LR Wh/OR-RT

snatch up $\sim 0.3 \mathrm{~m}$ fo deag $1 / 2$ " $p$ to us uof lom opin $12 \mathrm{~m}$ York Gum

200 tamnch vs. In 2 York guen CHT \$ - 5 m-undushinbs - 0

$201 \quad 1$.

.. watriler

202

203 v. upen York zoode, w Soun mad-sT. mod \$stribs gr shrtosuse

204 .

2as

d)

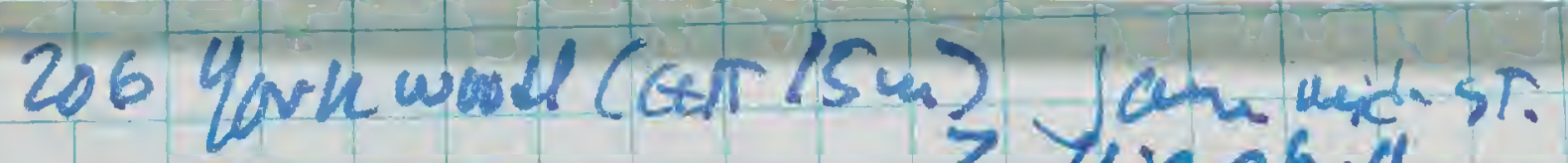
open us zweebil

27 ..

208

" or pulwh

2 Bee-enter

41 Quelwhith forlan tha feraging

o) Surtan leaves ding Lom $0.5 \mathrm{~m}$ or

c) "diag 12 mop in is u qork

(1) Suater muak kei Bin fro

2" dizy. puch lom up as $15 \mathrm{~m}$ gum

(3) Hf deq up fordyiz" to us

leves 1 Jam $21 / 2$ m upis $3 m$

209 open York hoo de w. 6B H Jan 11 midsty - Modtf shrobsin us zuieabill

210 Sam/hatere orch CHT $-3-4 \mathrm{~m} \quad 0$ open us

211

$\because$ mod Shrubs

isits

ix $u \varepsilon-0$ 
Nested a Ruf year

(1) camen of weatien at hat caver of Weats, whue 1

toeen him severs tomesin toly and Aonust. Perry has Let up a live of mist vets and played tape tar 2 minutes te caun ritgleat o in wlo wak prenimoly. sound, wrules once $N$ Mica, qot Couqht. Banded Blue/Marve, Wh/mutu Ried Butcherbivd Nest in grmect $10 \mathrm{~m}$ up in $15 \mathrm{~m}$ thes. Bornas havgi around

Surver alons 5. Margit of curd moni E. 7 trom SW conrer EDGE min E.

य2 2 pied Butchubira (vesting), galahs

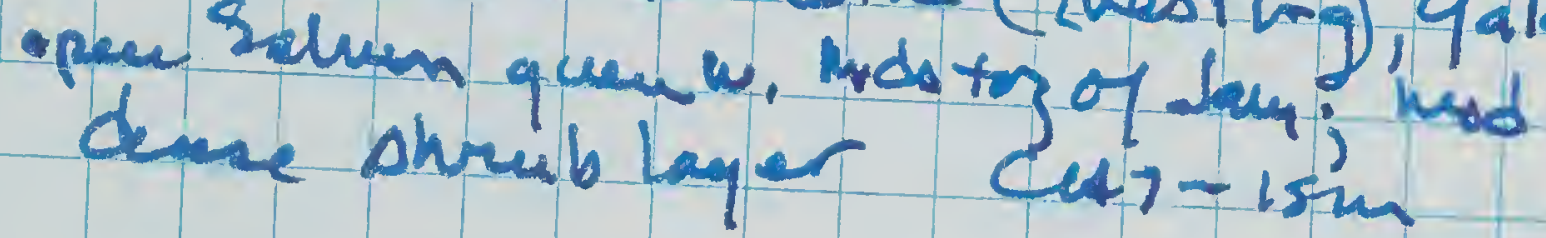

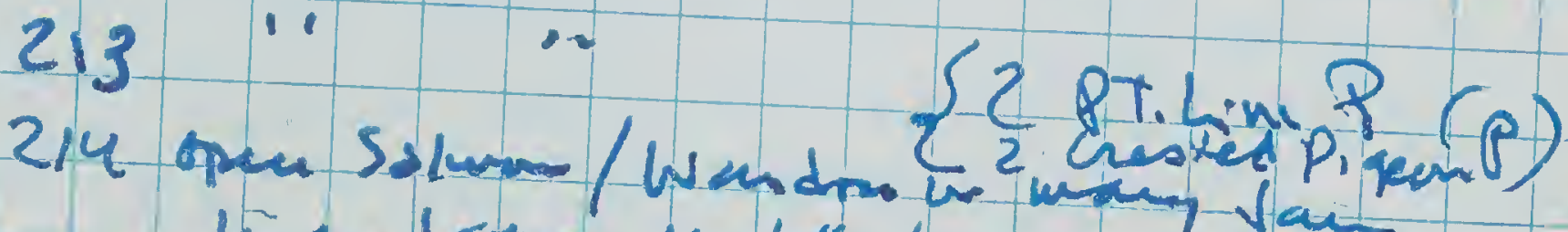

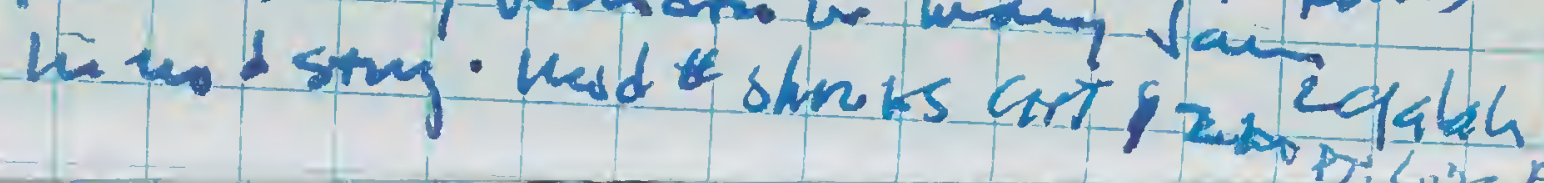

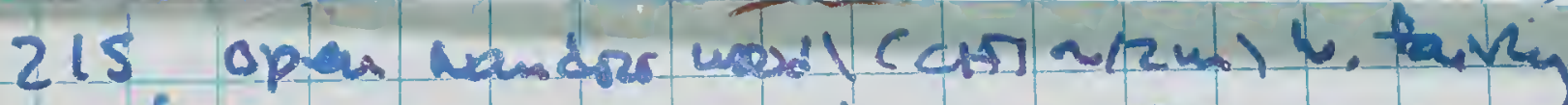
dere stands of saplipe, dam, casumina, ex. in as

$216 \cdot-$

c

217 Jam/sapliz ord w. W th shrubs in us YTMimar, Pr.hincP(p)

218

- - - 0

219 V. upen Waudor wosde. W. Faury dene Mdaton 7 dan 1 glun sapling; node * shrubs CHT $12 \mathrm{~m} 3$ Y TTMier

220 Jam / sapriz wordl. CHT $16-8 \mathrm{~m}$ andushrubs 3 Phline $P(p)$

\begin{tabular}{|c|c|c|c|c|c|c|}
\hline 221 & i & - & 从 & $\mathrm{CH}+\mathrm{T}$ & $25 \mathrm{~m}$ & 0 \\
\hline 222 & - - & $1-$ & $1-$ & . & $=$ & () \\
\hline 223 & i & ' & s & & $\because$ & 0 \\
\hline 224 & , & ? & ir & & $k$ & 1 prLn \\
\hline 223 & $\omega$ & $\therefore$ & $\therefore$ & - & & 0 \\
\hline
\end{tabular}

Thes bins we ahwost to SE Cona heare do alng w bonde

226111 ile Eysminer $227 "$ ". " L Lehoxe 228 open Unudbo uvoll (Cotr $8 \mathrm{~m}$ )w, bots of Jam a moddussity of shubs 2 lusure waq tai 2 Red wattlebir PT.Lin P. (P) 2 Y-RT Thil

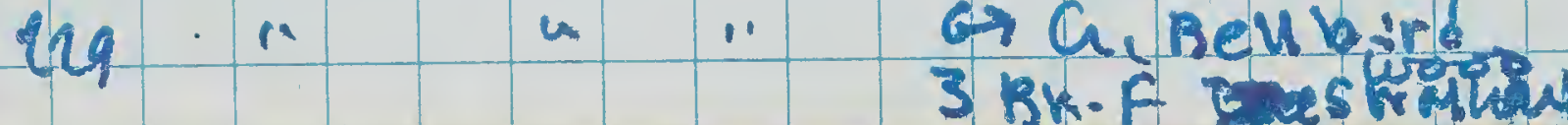


250 r.opan wardro as mod. * seattend. smubs- cur $10 \mathrm{~m}$

Richounds Pipis (aredge 7 proddock)

INTERRIOR WeTh HEAD INT GIUTERIOR

231 miller strob farey ceme $\mathrm{CH} 2.5 \mathrm{~m}$ por Ref whisteers, 9r. Sursize Turnoh

UB of Ray whister-Sinter diag op $0.5 \mathrm{~m}$ f. haviz. 11. $p$ to us branch 1.5 un up in $2.5 \mathrm{~m}$ hater bus

232 $\because \quad \because \quad$ BrHE, Sing HE

$233 \ldots$... Br-hesded HC

234) $\because \cdots \cdots 26 k f$ wordas $(p)$

235 dase Casuarin Thichats

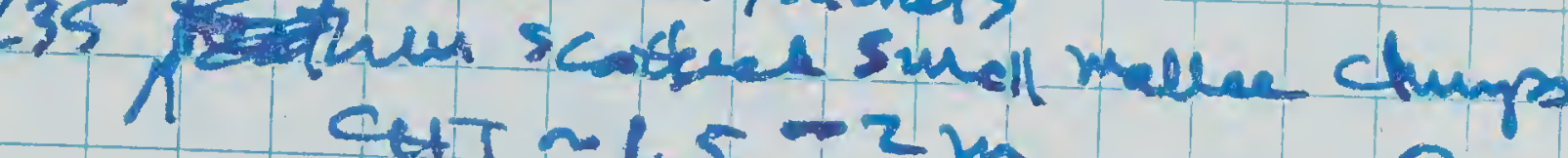
Cut $\sim 1.5-2 \mathrm{~m} \quad 0$

$236 \quad \cdots \quad \cdots$

$237 \cdots$

-

238 hatunog. heath (Grou., Cas., the kea, et.)

sone mallec 1 bead lorae gums 2 gaich Sinc HE EN Cormin Bronze

239. Guu saphi Thichats and lact

colt $5 \mathrm{~m}$ surubs dene 2 Weabills PT Line P ( $p$ )

$240 \quad \cdots \quad \because(C H T-6-7 \mathrm{~m})$ I SeNaHE

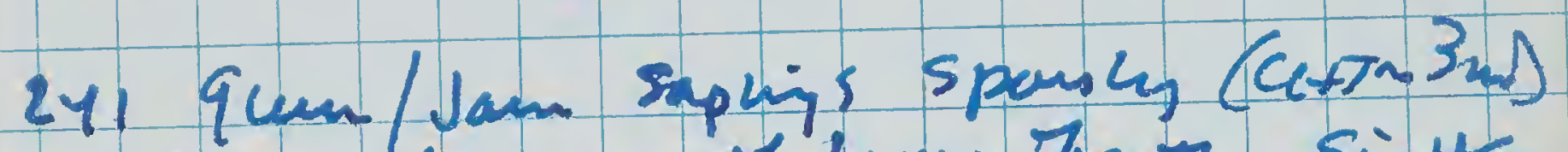
sentuld in avea of divar thath sighte

242

$-0$

243 qum wrodl Coỹ su ar dense.

heath us

o

244

Smate

2us Eue wosot (Sulums) Cost 15-18n

$u$ uthry 1 bur, guensopups

nod. douse slenebs C. Bronzewing

\section{END AT $1730-$}

Spp lere at 5.end 1 weras

Pied Butchenbird, Rul. Whithe, Y-F Miner,

BuHE Sirg HE, Red Wattlebird, Richoud's Pipir, Bk-f Wordswallow, PT.Linc.P., galah, C.Bronzeing. Willie Wagtail, Beubide, Grshr The, Br-head.HE Wecbill, Yeu-r T.bill 
27 od 88 KoDj Kojjin Regenn Gils clear, cork, alm

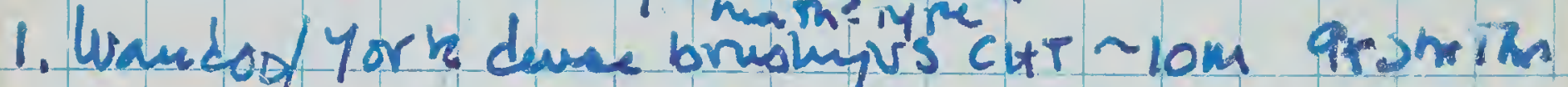
or Ruy. Wreitter. Ely. Parrst, PT. Limaile P.

2. .. 3 weestll

3. ‥ "CHT $n R-15 m$ US mod dume

2 ch-RT-bill, $\sigma$ ra R.C Rubl, galals

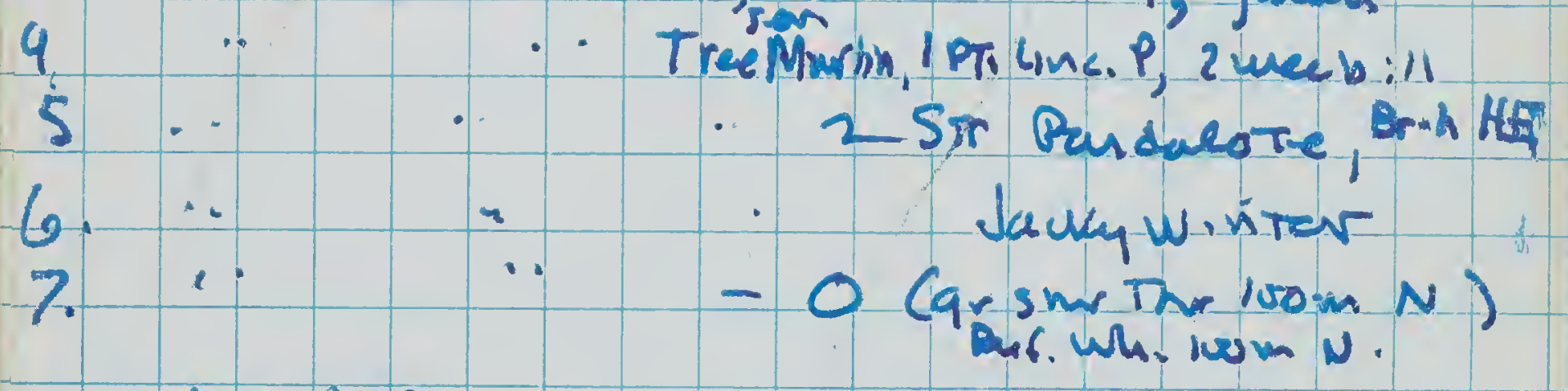

8. gam saphings $8 \mathrm{~m}$ high - nod * shrubs-sav. Weetsills

9 ." ". fairly deuxe Cas.us - 0

10 .".$\quad$ " mod shmubs - 0

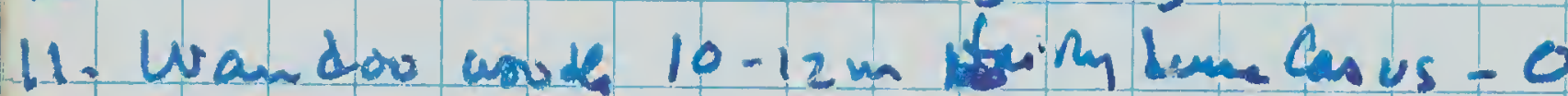

(arsur Tun $100 \mathrm{~m} s$ )

12. ". " " openbuchus 0

13 13. . Prywh. looms

19." " " " 2 weelaill an (smpatches of las.) Ry whloos $N$ ix

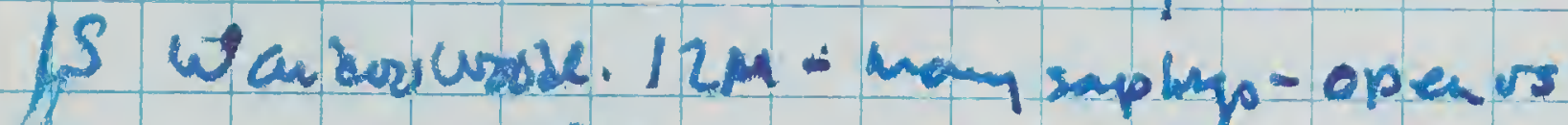
P. Limcoparrot Weebill 16. ". . " ar Shr Thr

17

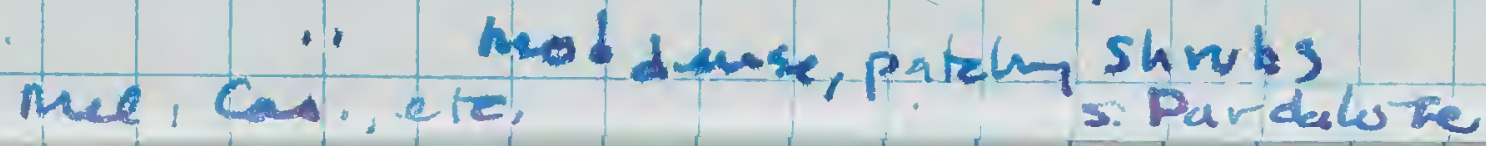

IE Counts Rur. Wh IIII G Gishrthr HHI II (6) (2)

18 open Wanderowrode, - md ishrubs

O

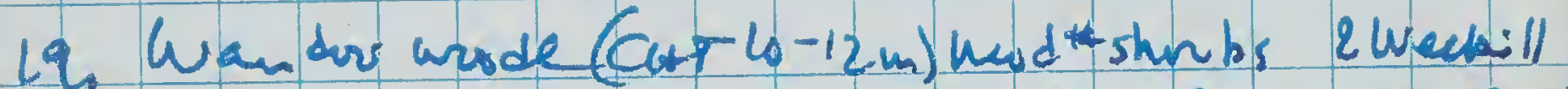
20. - " $"$ R R.Wh. 2 smipard.

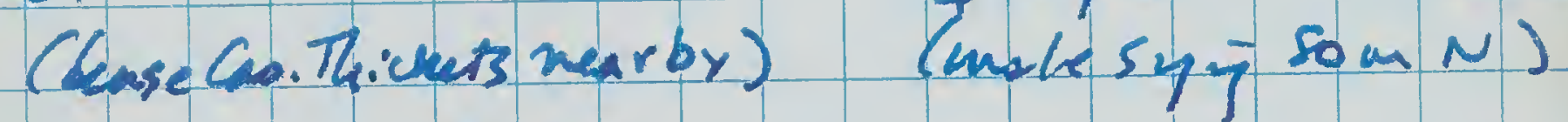

26. Salum /atik gan arodl C CH 15-18 m

open us u. lour suatend sarrbos - O (2nllee in tenem- ced bessmin)

22.

Cr. Currawona

23 Mave vordh att $26 \mathrm{~m}$ V. opan 45 - 0

en Wandre wrod w. mukemids -open us - Wrels:ll

25. dinse vallee w. Seartend Strum gun Diff

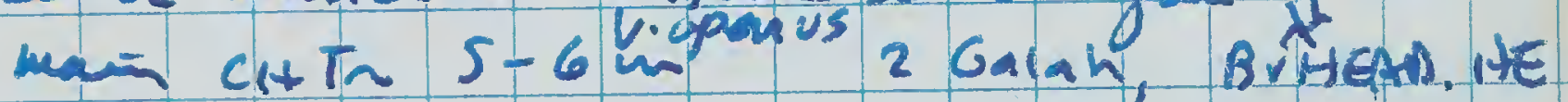

26 Sdlum gam uord d (CHT 18 w) w. Lun Make midstom-vopan us $2 y-R T-b i l l$, Aredills 27) Sdlu/4ak (to / $5 \mathrm{~m}$ ) W. fuiry dum

make MS and Mot shmbs Ia 4S - 0 (Ry whster $100 \mathrm{~m}$ NW)

$29 \cdots \operatorname{cit} \sim 6 \mathrm{x}$ unebill

$2 \because "$ atrolom 30. Dease Mel. - Grex-hanth 2 high

31 Mduse" "hath 2-2.5m 


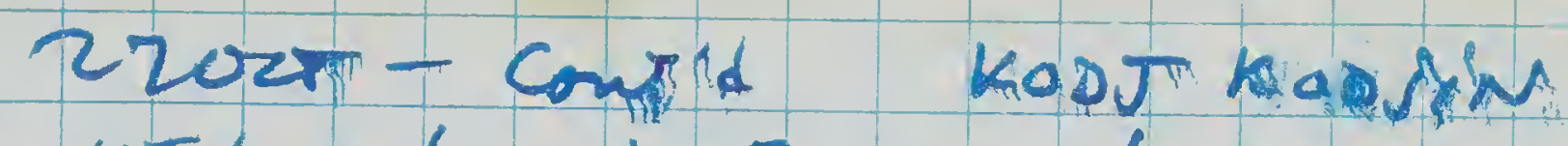

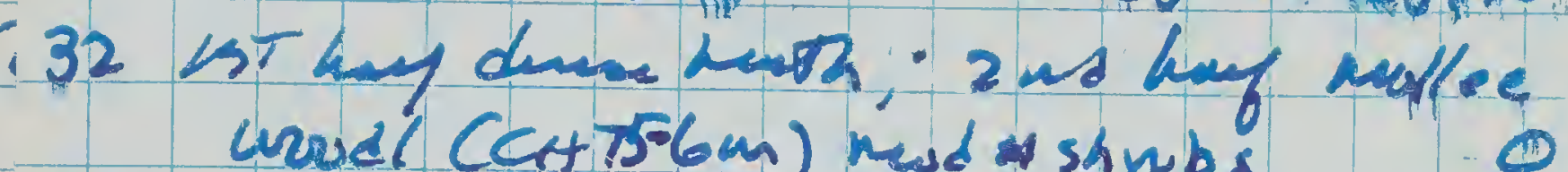
133 like cat $12+32$ o 34

$: 35$ us tairly open, soudy 0 36 - mad shubs - 0 37 danse Mel Weath $C_{4}$ r $1.5-2 m-0$ 438 dewse low hath ur. Dry undra, ete $\mathrm{L} / 2 \mathrm{~m} 6 \operatorname{sit}-0$

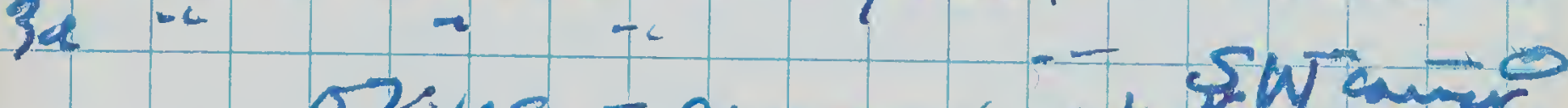
ovics - euryed at Showinger EDGE of kerij kogin-hend E. 40. hefeng kesargun, nosily $4 / m$ kigh but 4 . clamps joreviller, as, ete. to 2.5m 5 uhror Babbla, ste

$142 \quad \cdots \quad$. 43 "ctr $1.5-2 \mathrm{~m} \quad 2$ wh-eard $/ \mathrm{E}$

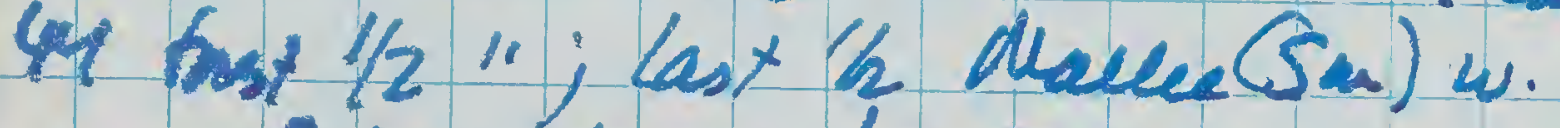
Gavily dewe keata us - 0

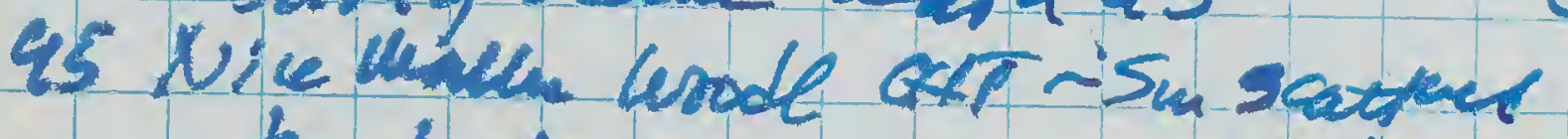

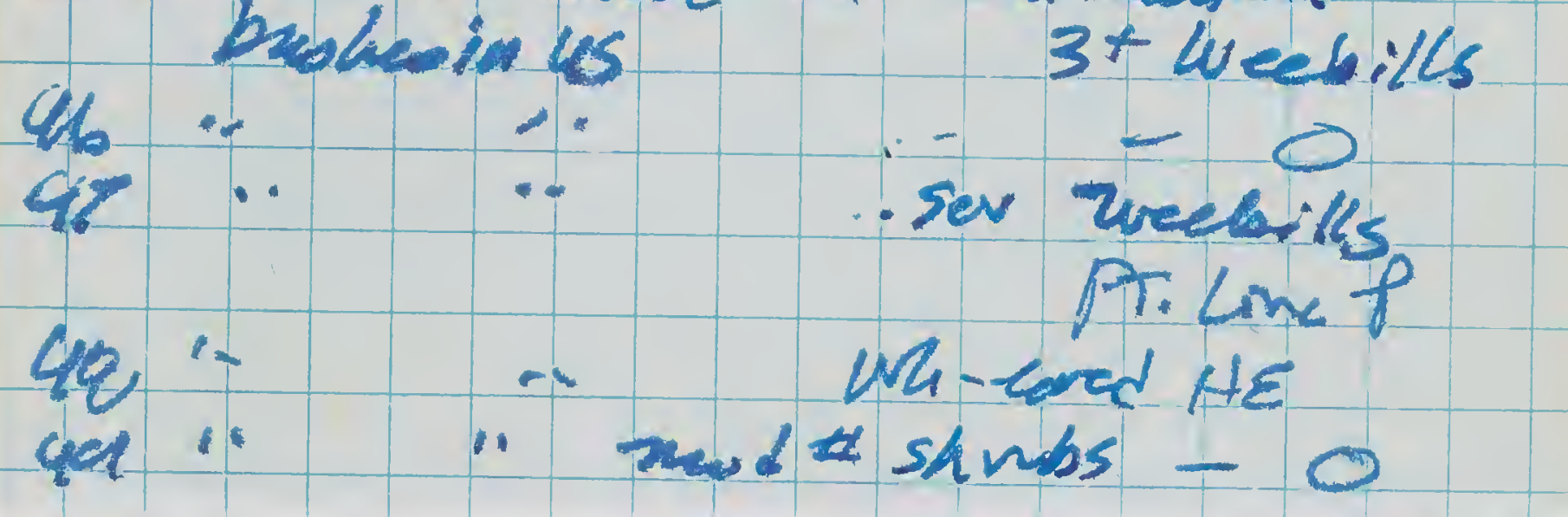

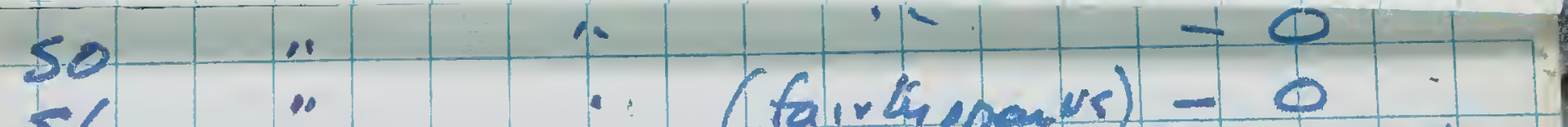
$5 \%$ ssow wacebll 52 i $\cdots \quad$ " 530 " 4. sestar lark quen $\sim 12$ m-scatad hubes - 0 S4.. .. . . . 0 55 .. Gaing deme hatk us-0 56. dease, divene hungun - qrevilla, Hakea, cumanis. Kedpes, Acus, ete. Cot $-3 m$

57 drmat a. sur malle to Ya BrHE Wh-everte

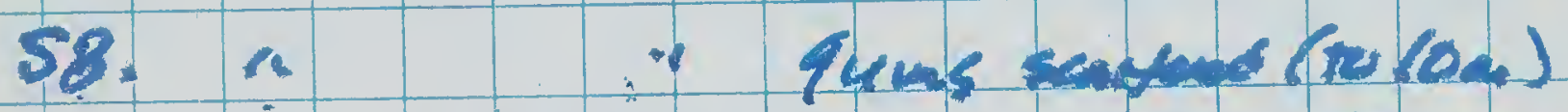
even $(p)$

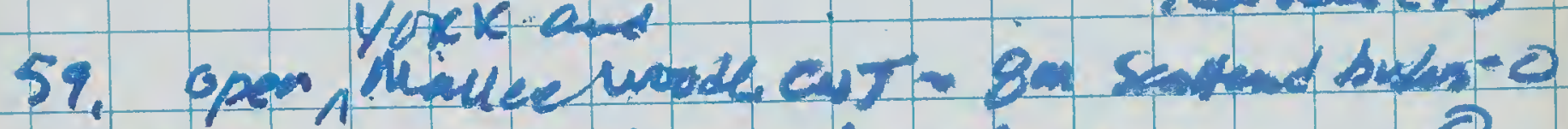

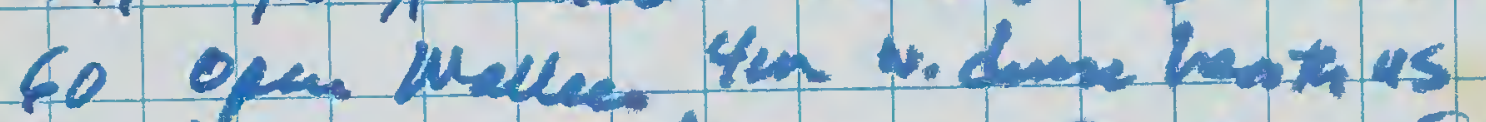
62 dene divene Lamyan 0320 S Brys

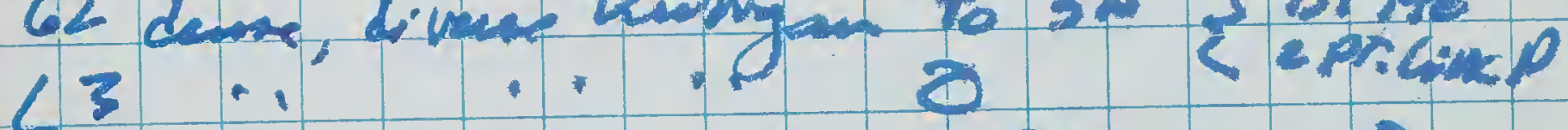

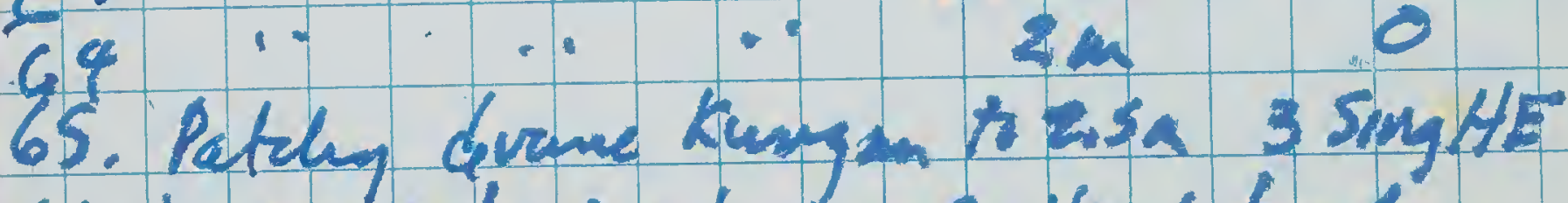

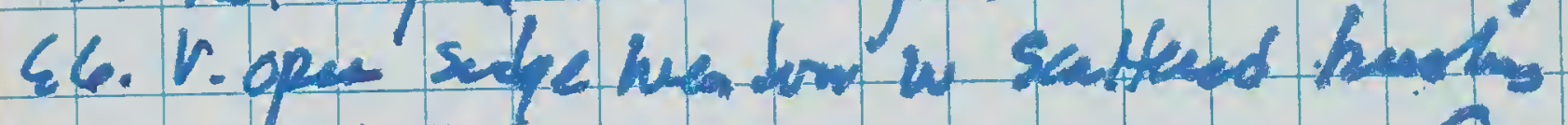
to $\sim 1.5 \mathrm{~m}$

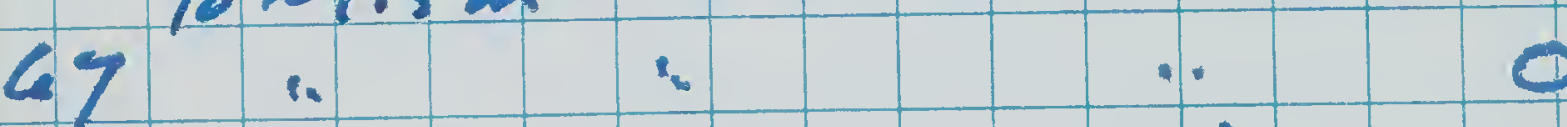

68 i $\quad \cdots \quad 1^{\prime} \quad 0$

62 - Eac. and Get $4 \mathrm{sm}$ m

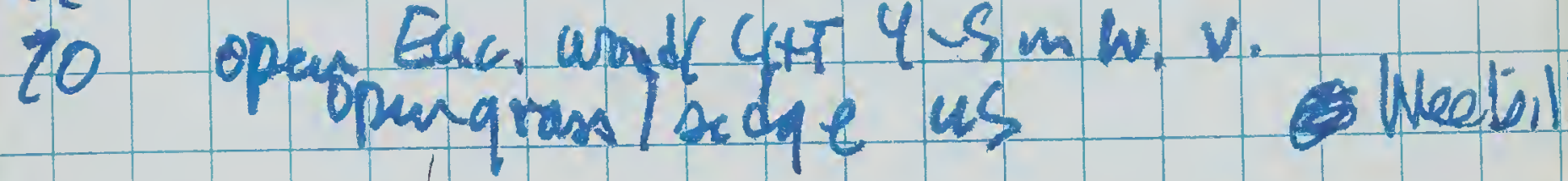
21 il 140 
72. epen forke uarde Cur r $10 \mathrm{~m}$ mad is Jaen orum mums, Ma smubs US - 0

08:30 iSE conven of Kody kdin EDGE TemuN. a lory boundry RA. $73 \mathrm{~m}$ llee woode $5.6 \mathrm{~m}$ mod $t \sin x-0$

\begin{tabular}{|c|c|c|c|c|c|c|}
\hline 74 & "ఉ & $\because$ & & . & & prilineplp \\
\hline & $\because$ & . & & $\therefore$ & Vte & sp. $\mathrm{s}$ m \\
\hline & [* & 4 & denes & & nobs & 0 \\
\hline
\end{tabular}
INTERIDR: This binjp ine to accens The ware $/$ porned

77 .. ․ .

Speedes sean ar heans, but not

appeny on ceuses: C. Bronzewing. Maypie

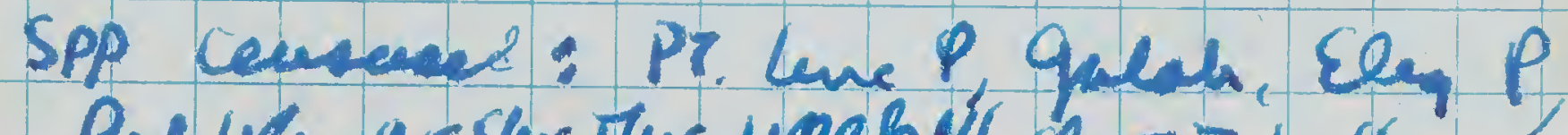
Ry wh, qrshr The weebily cher T-bill,

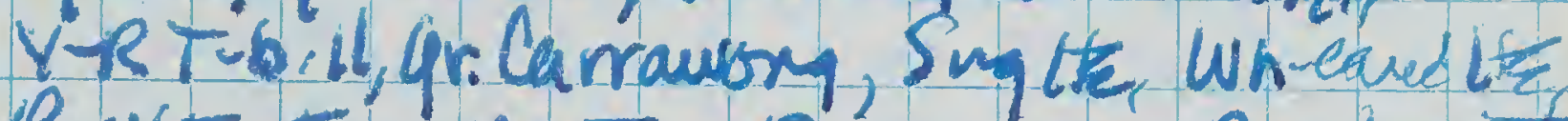
PrHE Tre mantan, Raven sN. Parduete RC Robin, J.Wonter Br.huads HE 1850

0930 wEZDS - w. Side wene water bipe ceits in prom INTERIOR

78. opeu Sal wor (Yock wrode. 612 - is M CHT) 4. Mob I Jam in MS and busken in US

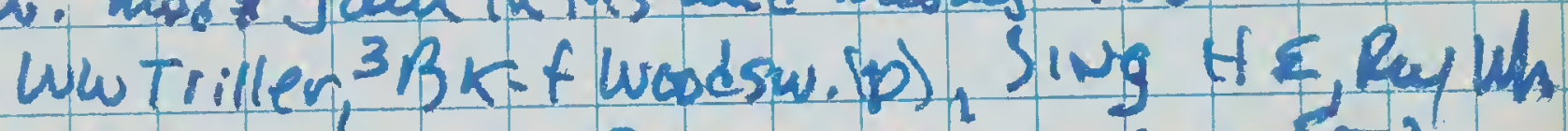

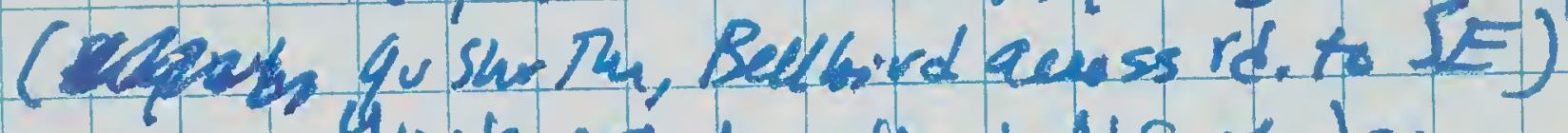

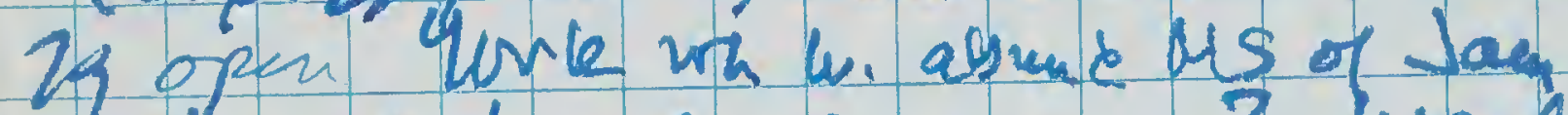
hod buabs in us 2 Wrebiel 80 tam orchaid (ceft $4 / m$ ) unstushings - 0

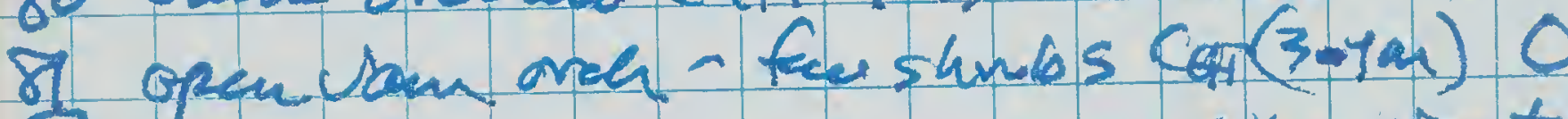
$82 \%$. W. Willic waytal 83 open Vam / Hakea oree ropales atr $5 \mathrm{~m} 0$ 84 Jam ach - quens co 3-4m - 0

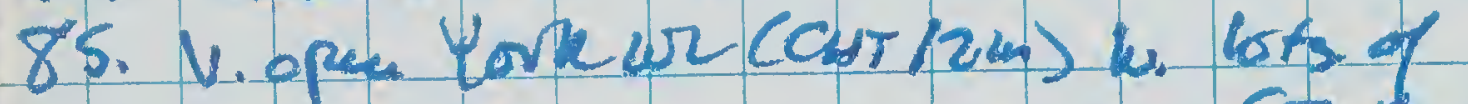

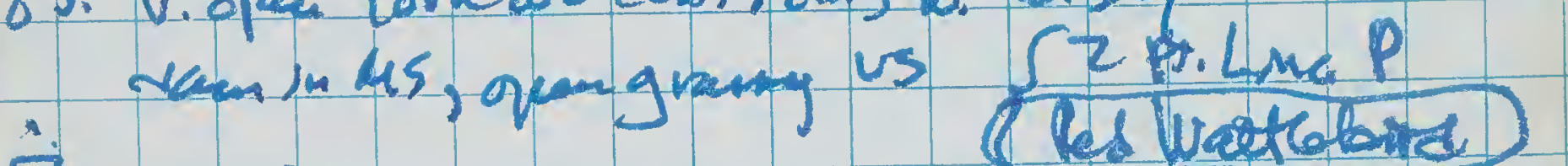
86

$87 \cdots \quad \cdots \quad$ \%

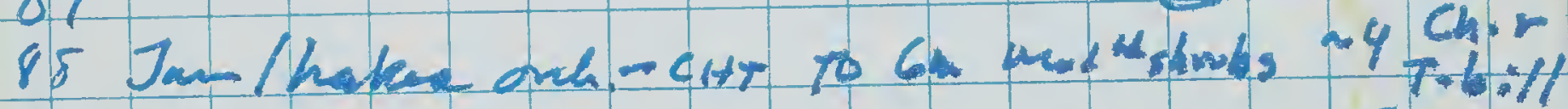
89 ,

(qushr $\operatorname{man} 75 m 5)$

qo... … Breadies HE, Wabill $q 1 \cdots \quad \because \quad-\left(C_{14}+3 \mu m\right) 0$

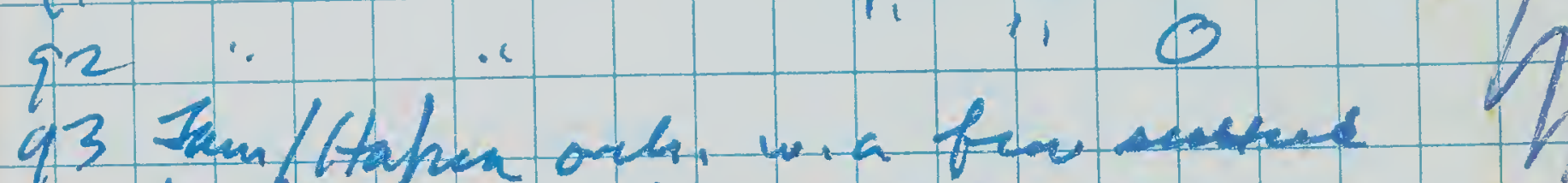
bu You han CHT $6.8 \mathrm{~m}$ open gma ves 94 opu 4ark wade. w. Jamil Cas Ms ope us Sev, Wechil (Now at edpe) 


\section{EDLE}

13 Niedqe of weeds

is. open york urode w. MS of vam /Cas, upa

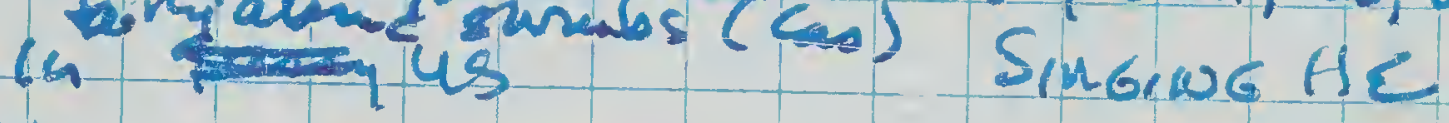
96 (Grsw- Ru $100 \mathrm{~m}$ Sig Rul wh tod)

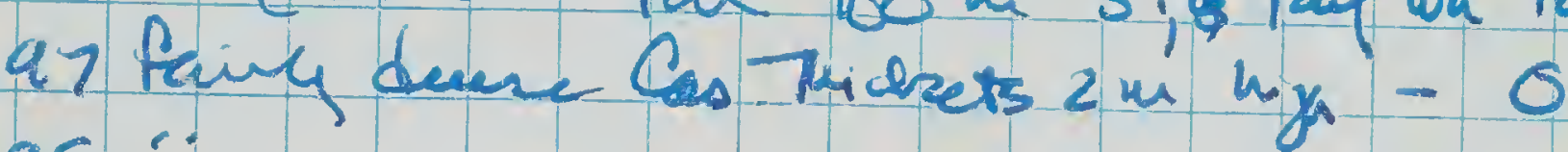
28 '

91 dam worde w. mad glumbs ins Corlm - O

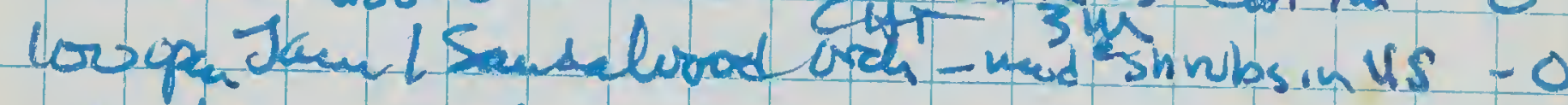

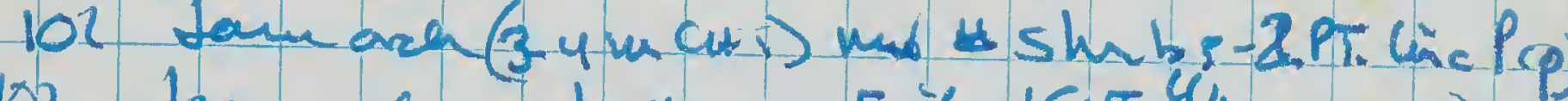

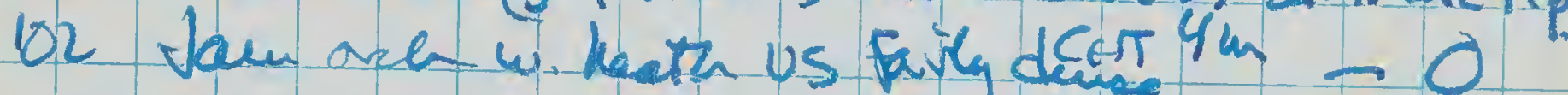
$1034 \mathrm{ar} 2 \mathrm{wos} d \mathrm{l}$ ( $8 \mathrm{~m}$ ) deme Ms of $7 \mathrm{am}$, orm 134

105 shmbs -

106

'. $\checkmark$ 10.15 (upper Nu carma - tarn E cery edge)

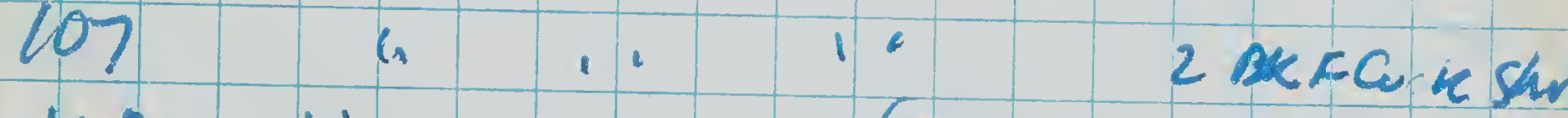
$108 \quad \cdots \quad r(8-10 m) \quad 1 . \quad 0$ loa quadyourcude. - modushrubsinus 2 Mogple herk 110 a ". " orperwhita, Sev. Y-RT-bills, qf triller ZBk-f woodsw. III $1 . \ldots 2$ z $\quad \ldots$ line P

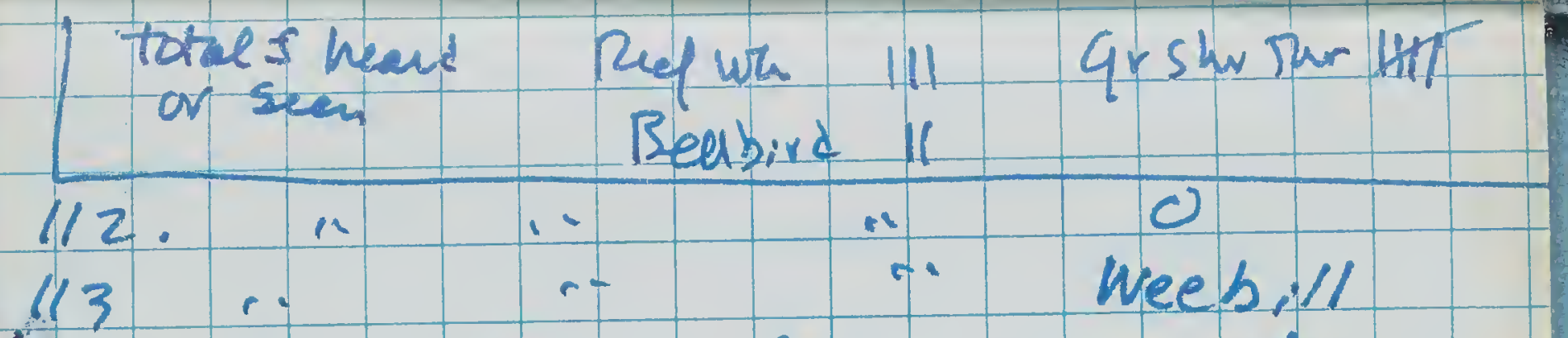

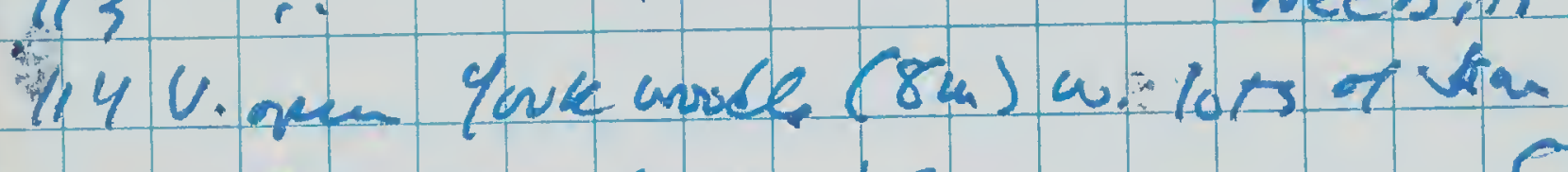
in Ms open quang us.

lis - - - - - a Triller

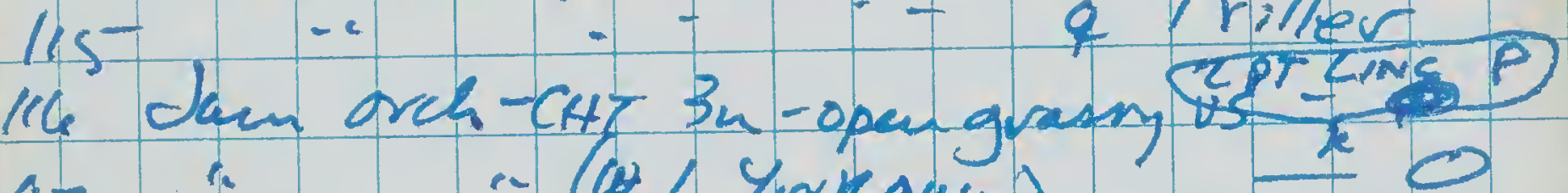

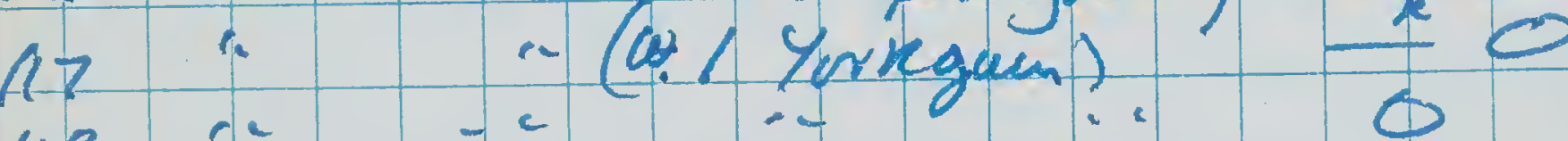

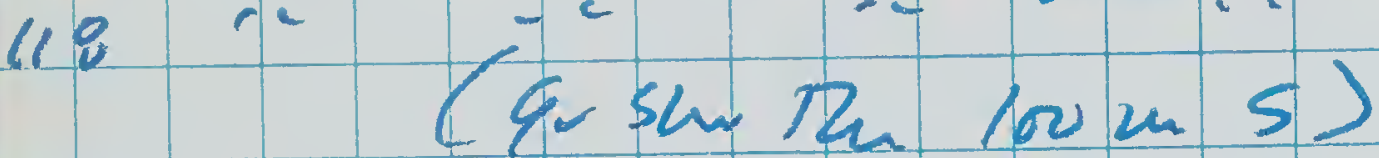

119 ic -c in $-i \quad 0$ 120 " " d IMERIOR SEGIES

121 YouK WOOd. LO-12 an CAT - Jam, bahen, saphí MS-mod shubs in Us weebill 122 open Jam wande CHT $3.4 \mathrm{~m}^{45} \mathrm{~s} 0$

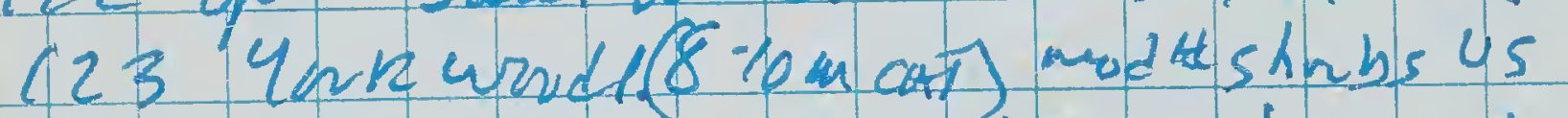
(2y upen York word 4, wh rt tam in MS aod shw bs an 45

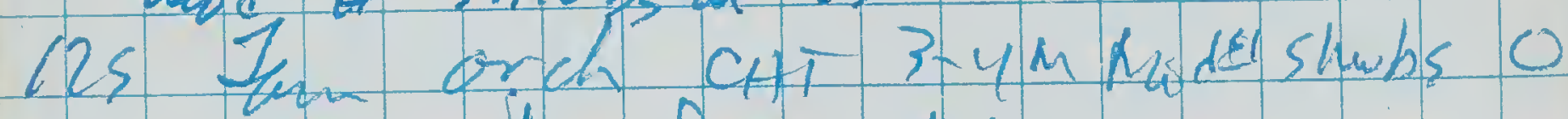
bachat rd-

9 Parallel to it SSW-stoy in m trin 


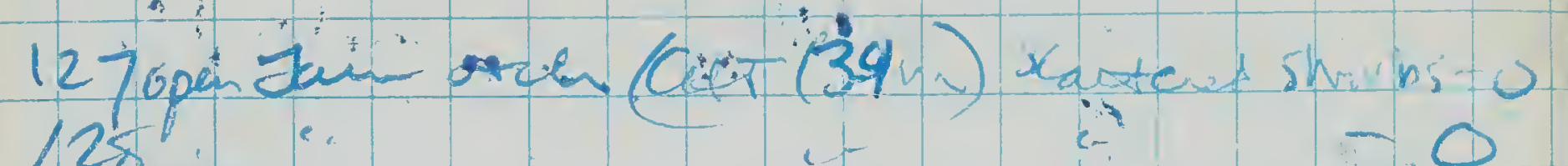
$12 \%$

(3) 1 (: 1 i $\quad 100$

132 V. upen dar aren 3.th opaus-o 133 open 4 ark wowbe 10-18 a Siatied $\sqrt{34}$

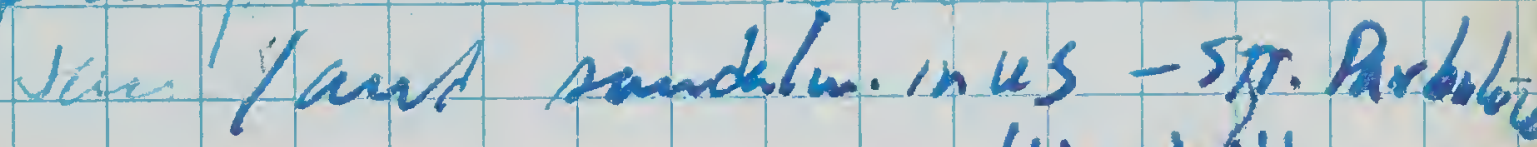
135 open Jan arch-openus - Weebll (36 York Goode. Tad sumbs mis

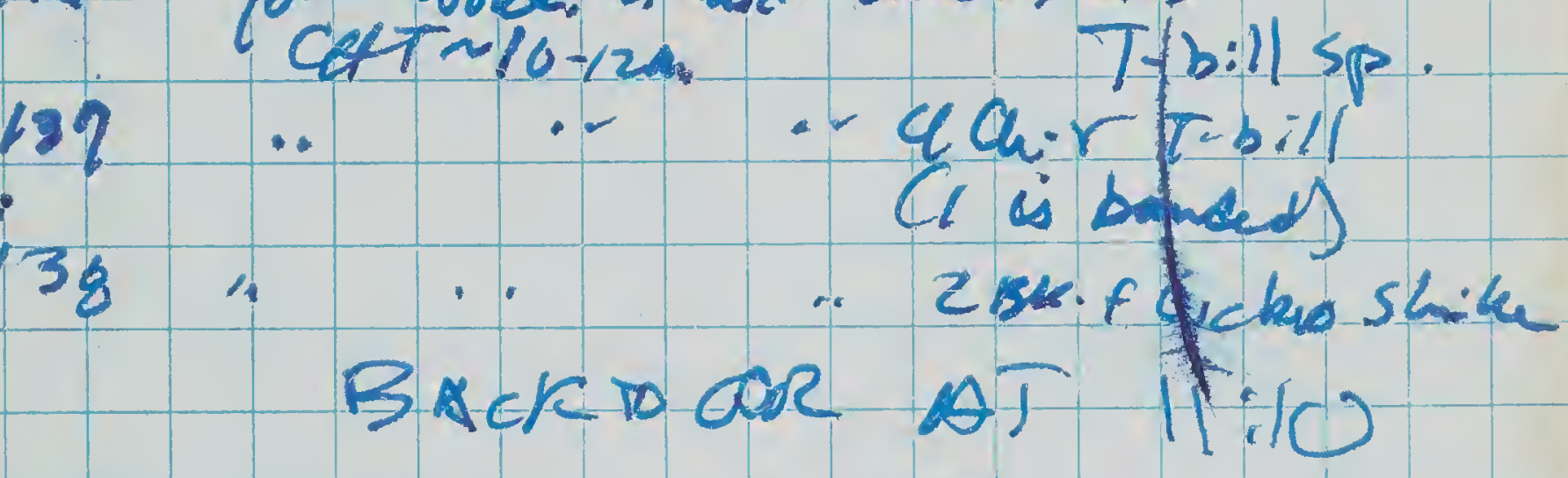

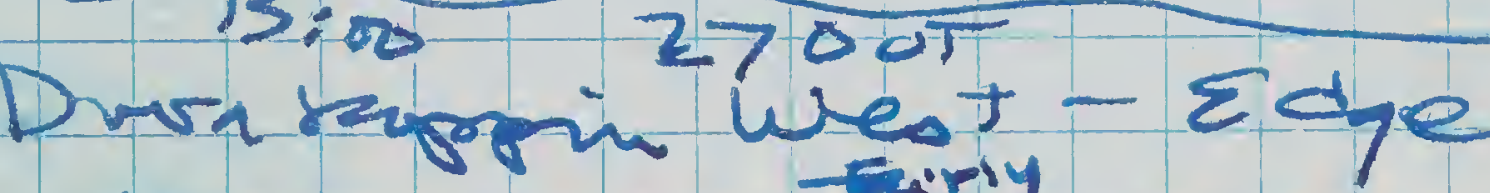
vam orch $\left(4-5 n_{0}\right)$ Eiriy dus shors

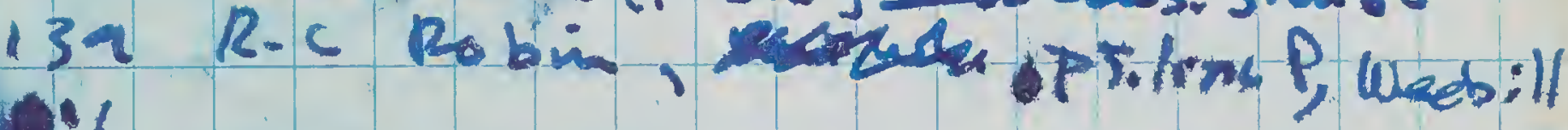

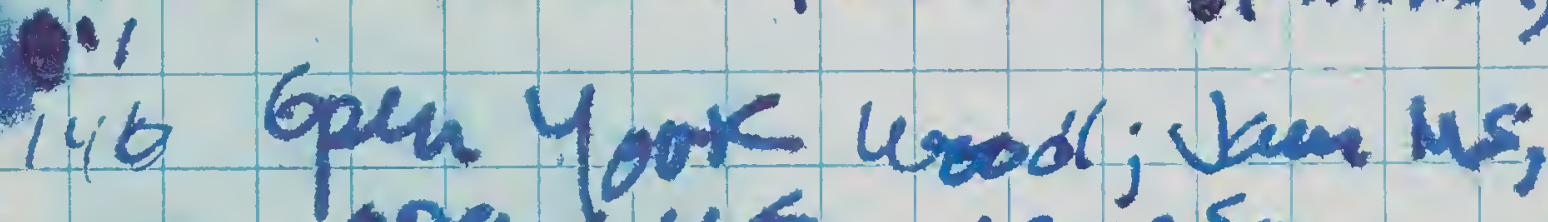
Toparlus $12-15$ an 0 $14:$

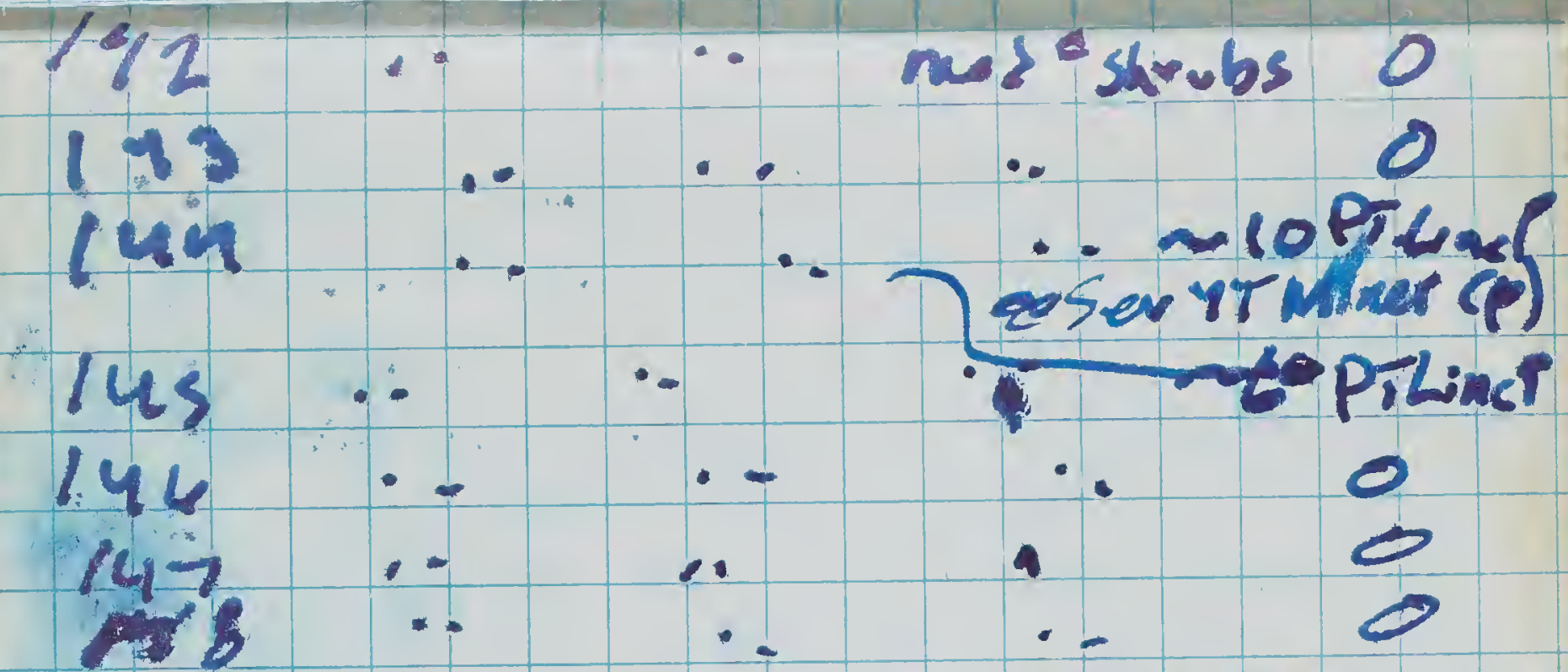

$14 q$ york worbe- $10-15 \mathrm{~m}$ - mod sthess - 0 150

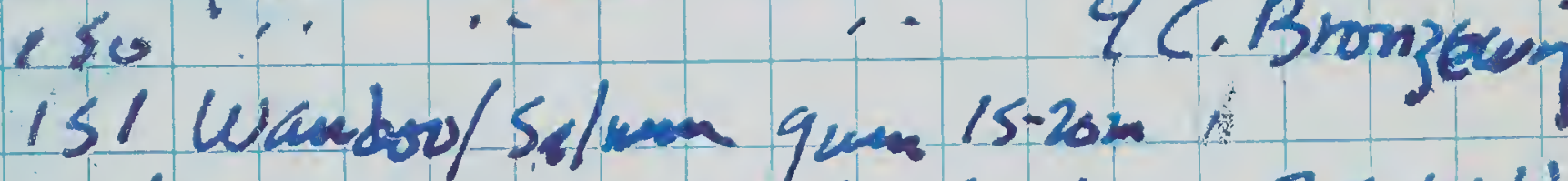

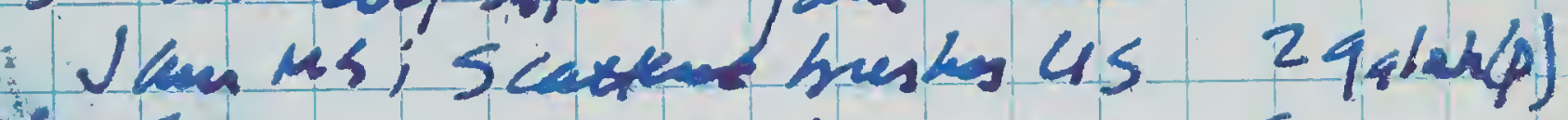
26152 - $G$ $153 \cdots \quad \cdots \quad$ inedersmbs 0

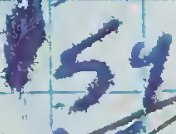
- 0 tetish : . . -

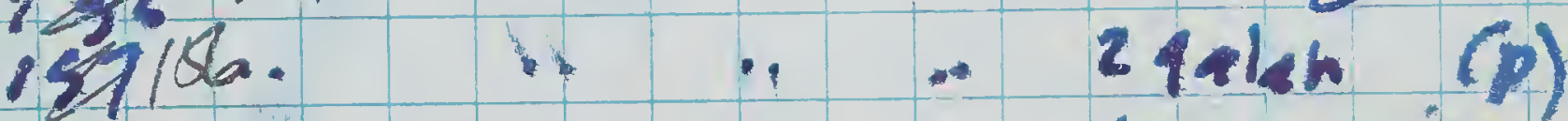
Firn $|x|$. ad be (Pr LancP $(p)$ towawd rd. hewe

INTERIOR

\begin{tabular}{|c|c|c|c|c|c|c|c|}
\hline & - & . & & & & & C $Q R=C$ Robin \\
\hline 158 & 1 & & 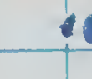 & & 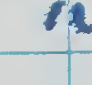 & & - 4 46 ye bil \\
\hline & 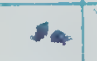 & $A$ & & & 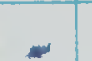 & $\because$ & c) \\
\hline 160 & 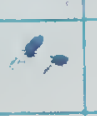 & & $r$ & & ? & - & $\rightarrow$ Wees.ll \\
\hline
\end{tabular}


162 Wandoo wood. CA4T 12-15m)

w MS of Malke: mod shrubs 2 gales

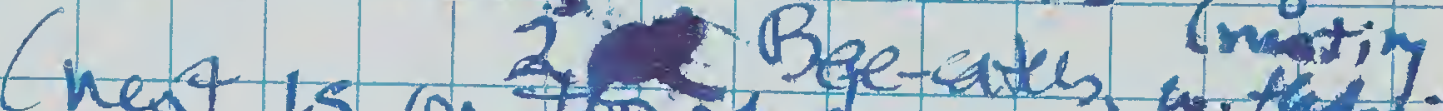
is on or ar. If limb of Salum gum $8 \mathrm{~m}$ cbovequid on 12-c4m thes

163 Wandos/4ork wri ils dami medet suribs US; $2+$ Sittella, 2 Gular (p)

164 opan Grudos/york worde CaT/2-135. atew smis shotad us 0

$165 \cdots \cdots 0$

ILle Wandos/Sahum Lorde 15-18 m

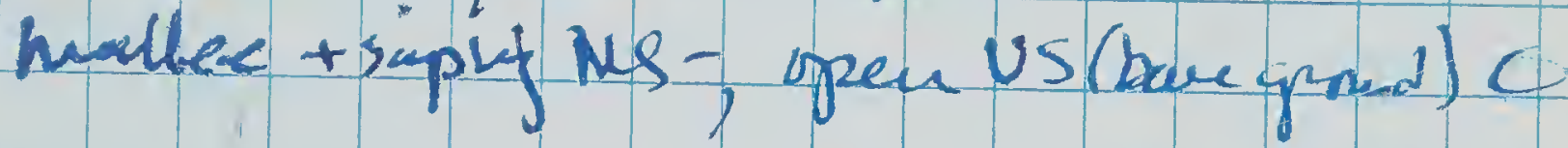

167

168 open York wad $8.10 \mathrm{~m}$

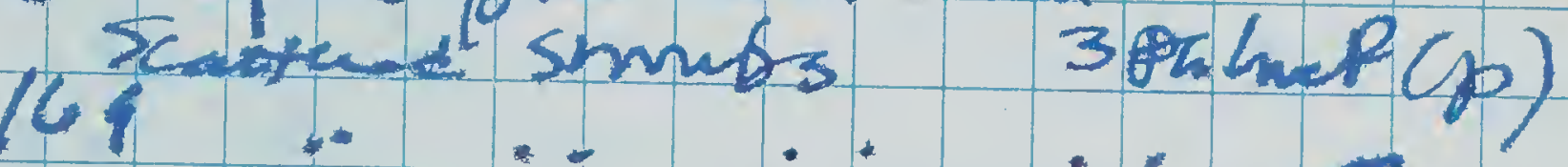

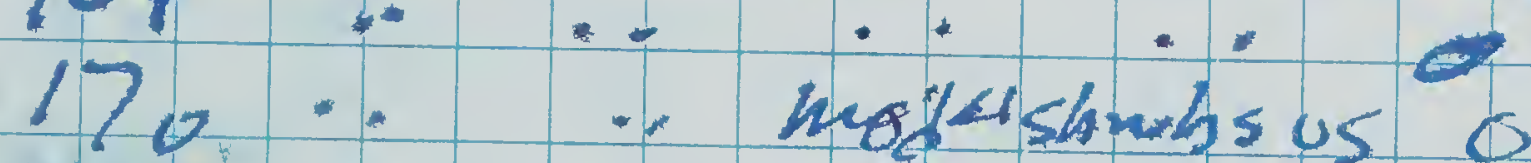

$171 *$ * * $*$

172 deme Cesuening Túclets $2-2.5 m$

173 Jam thind! 3-4n w deuse us of Coscreviona 4 PT.hine?

$175 \ldots \ldots$

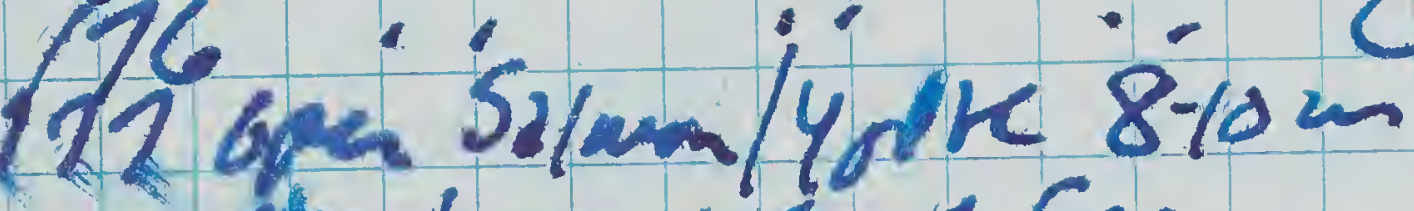
id Malke wovt 5 - Tin

w. dise us of melalenca

179 ... 2 Br $F$ 180 15T hal"; znd hall dene threhers of Cas. eto. w. ocr. enengert mallee IBrte, 4Ch-RT-bill Fuint at 1520 Pog. Writcur

Durakeppin Cidot-k. Side avess

trom Piptanver's nowne

SFout S. fiem lvanliar I100

IVTERIOR

181 openhando Lndi $(12-15$ s $)$ dene shribsin $4 \leq$

Q3 Vouto/sehwa G-ienne

E? lik

Buetatin

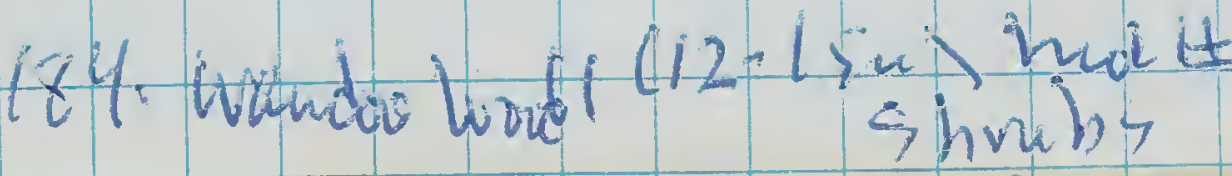




\section{I-1,002 hine)}

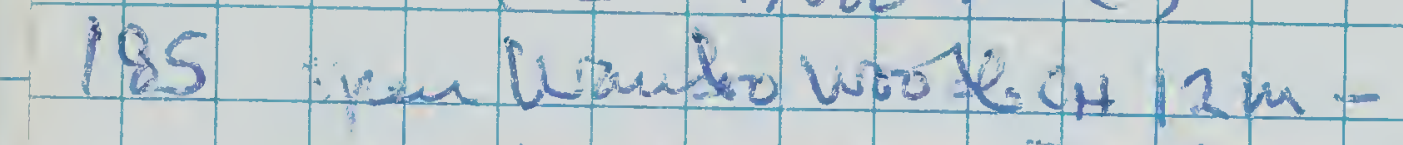

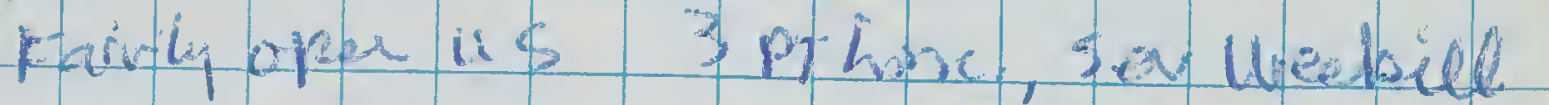

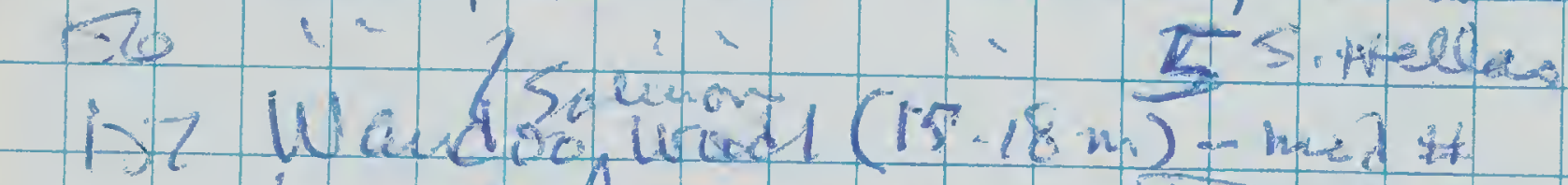

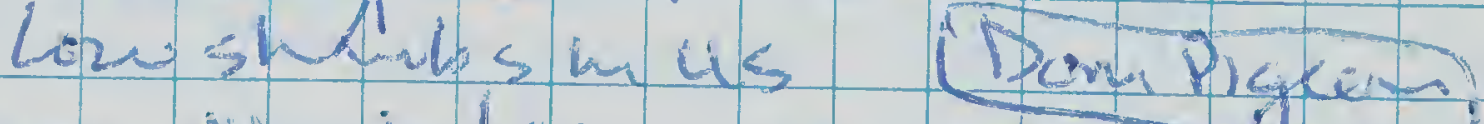

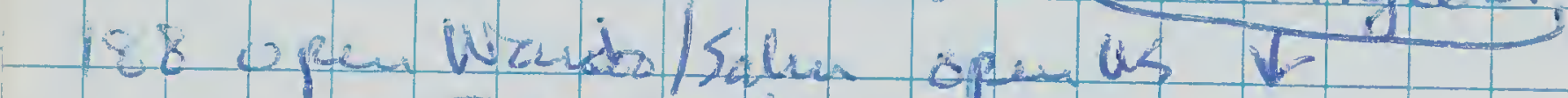
(1-1-1000 heie)

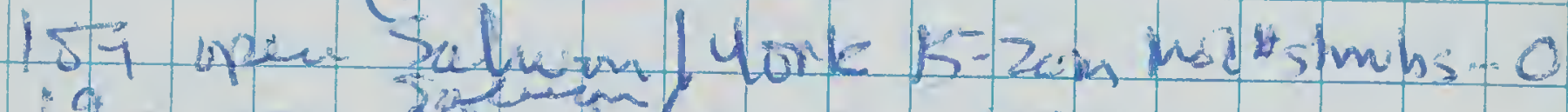

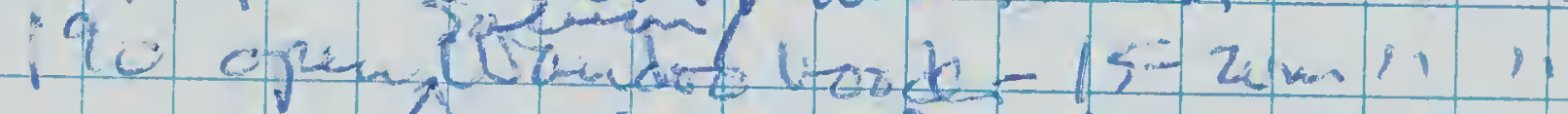

1 i) Waderuendi lisu

Scatard labshoru us - 0

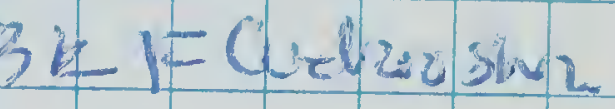

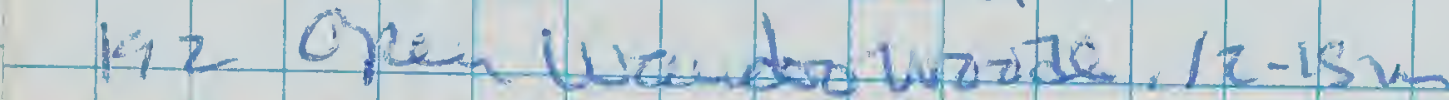

una shrubs us

(G 1000 her)

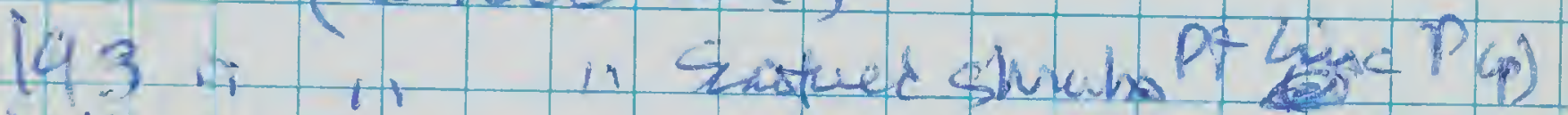
194 is in. A 4 P

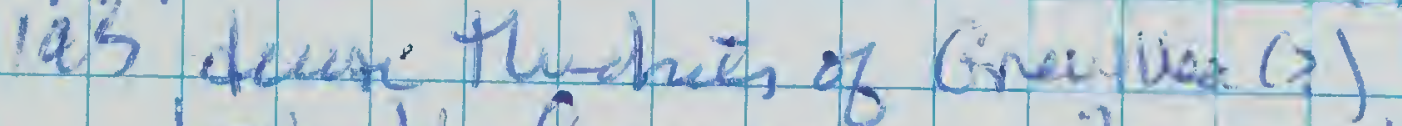

couks like Caucumana Zima

146

ipit i

197

$1952, \quad 7<m$

Ma Malle S-7wn dene shuh us

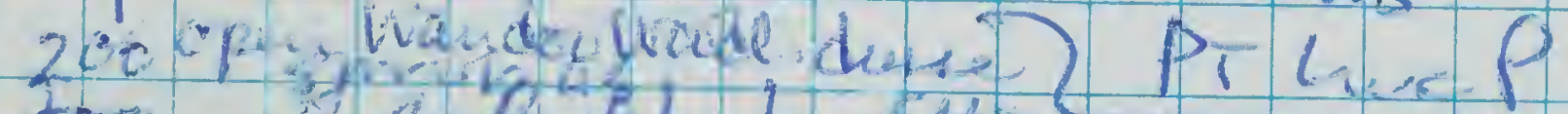

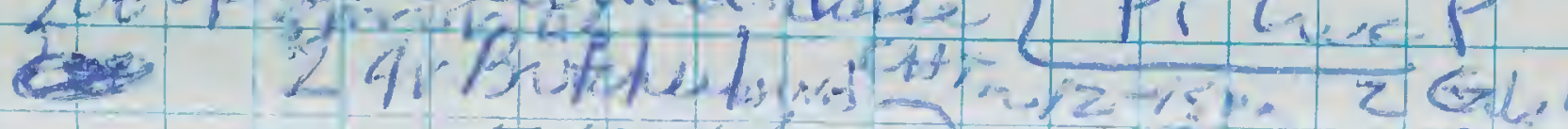

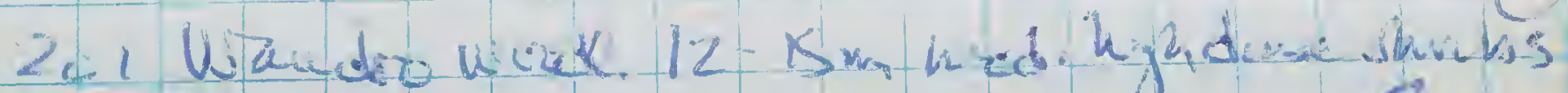
$202, \cdots+1, C$

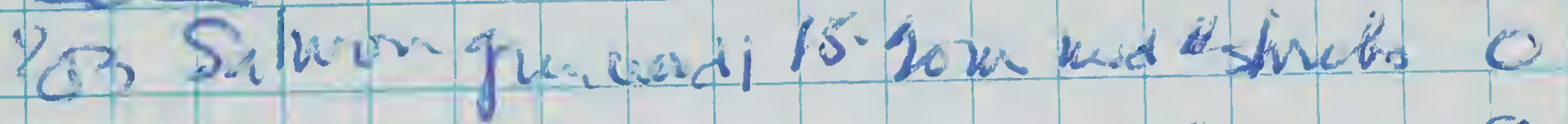
$2041, \quad D-1020 k$ hew

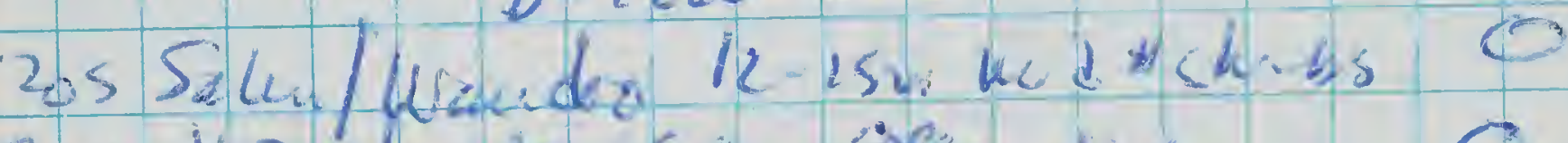

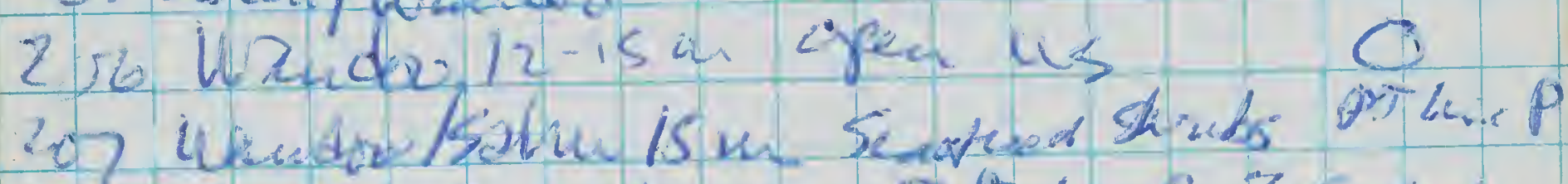

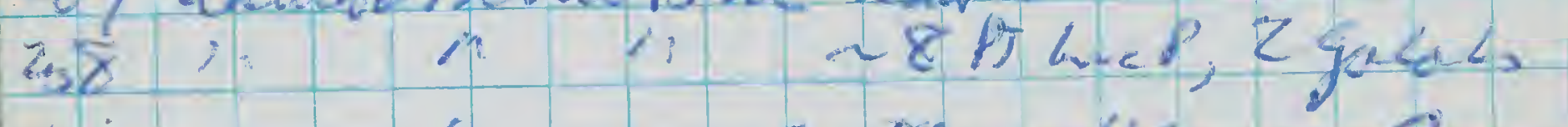
407 - opear lis o EDEE (cht over tord have)

20 Vapen Wando worll $12 \mathrm{~m}$ nora a deras stimebs 3 prling

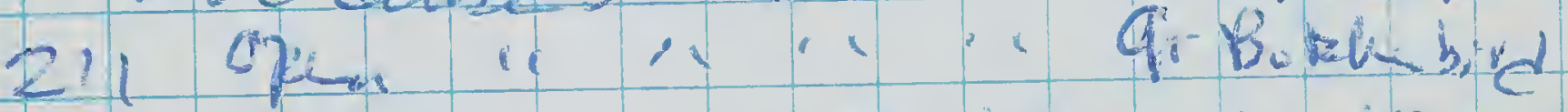

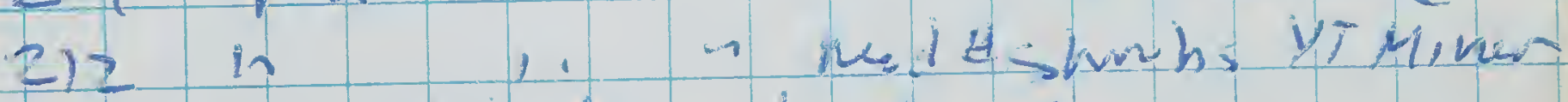
213 oper Salu/hiando iz-ism

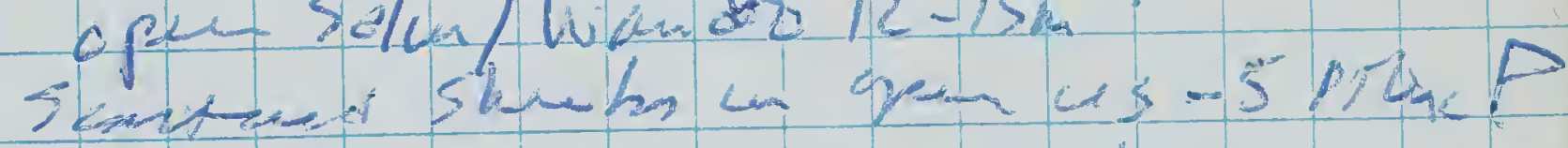

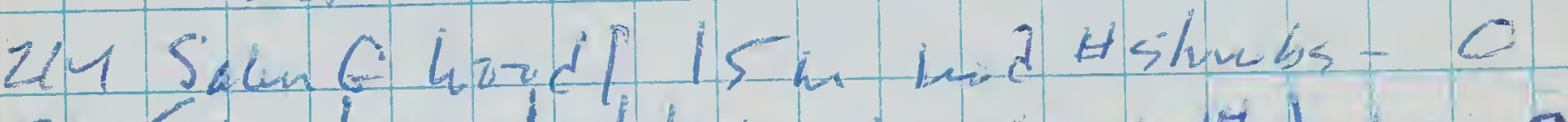

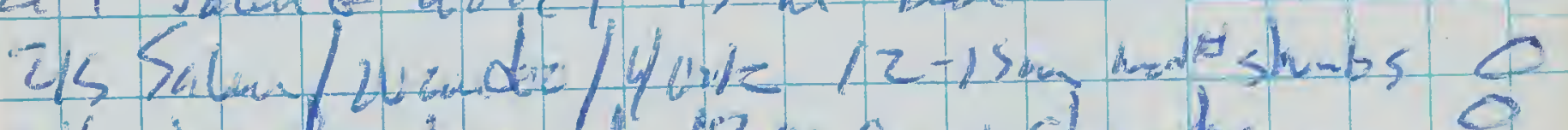

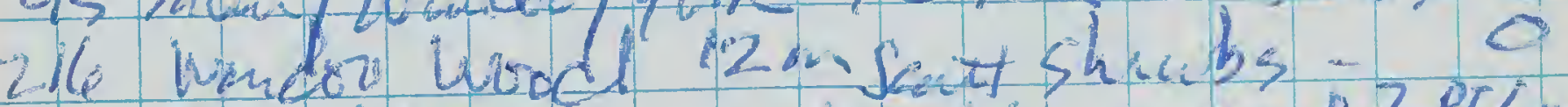

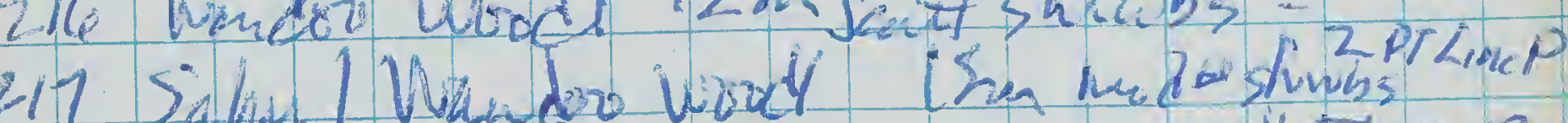

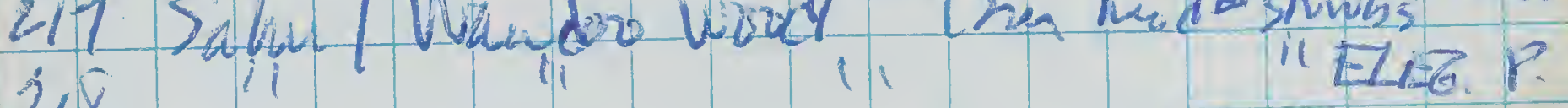




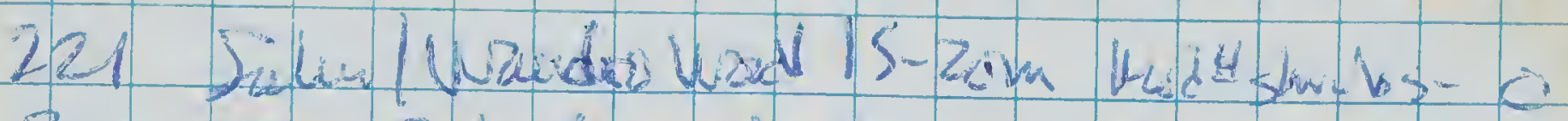

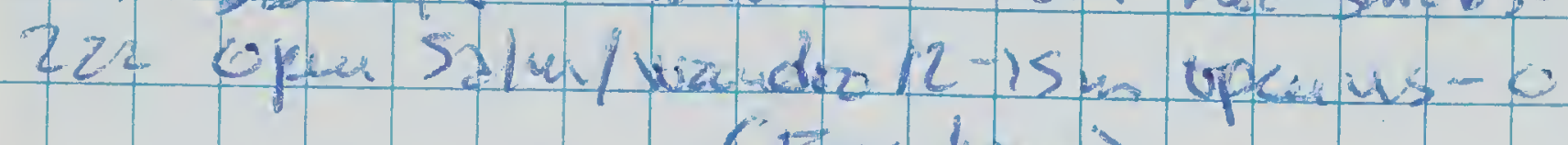

223 i) (Fex here)

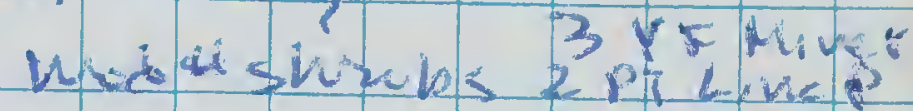

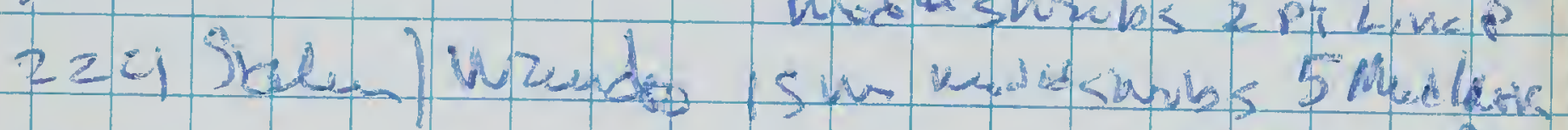

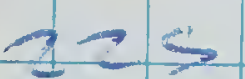

226 reptline? 1 or bure

227 Ijaloh

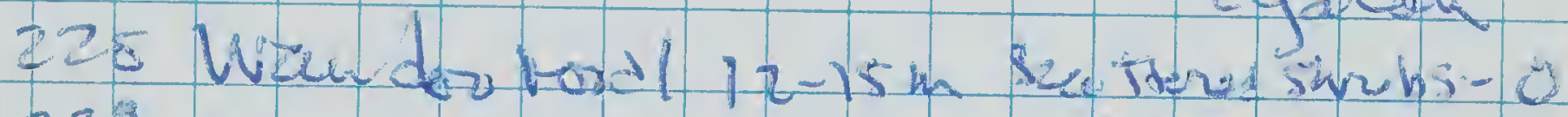

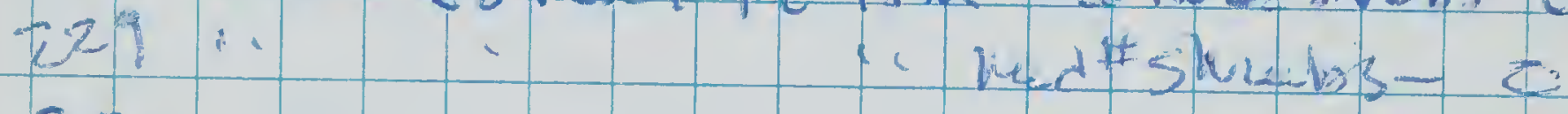
23

$2 \rightarrow 5$ peiand 15-zom in a-c

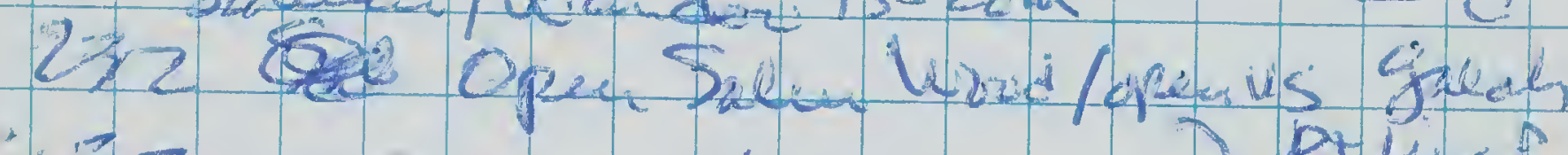

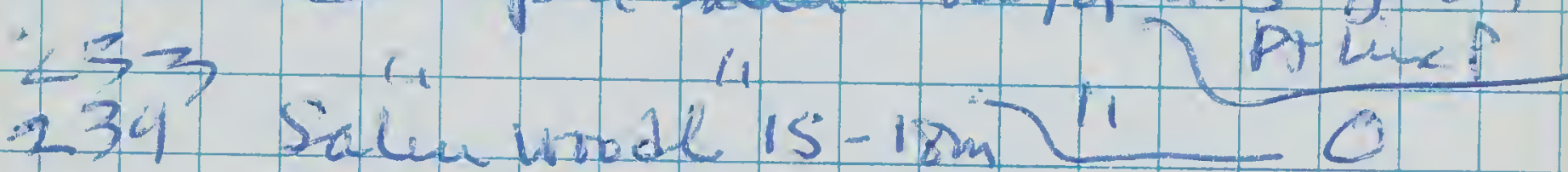
onwa sumbs wo us- o

235

L'igalal

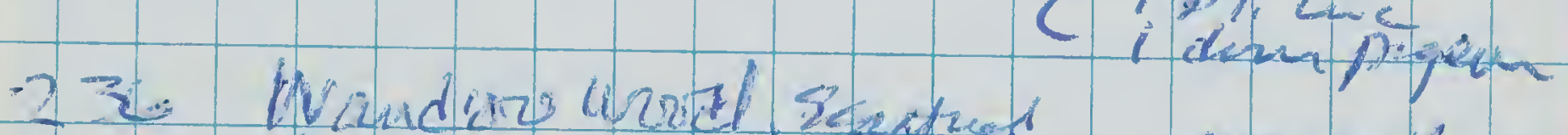

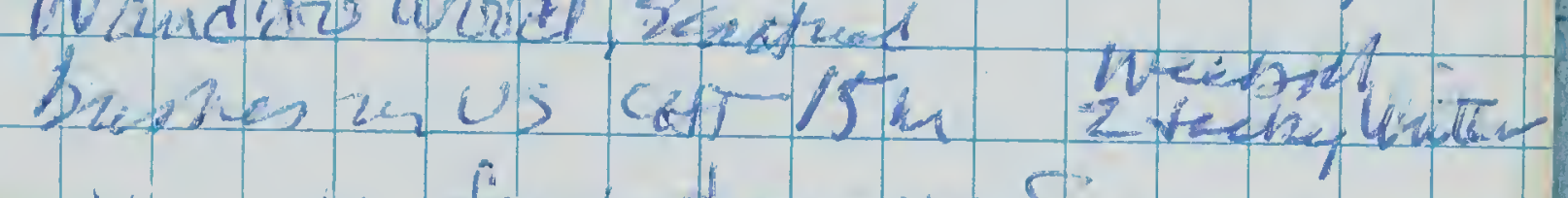

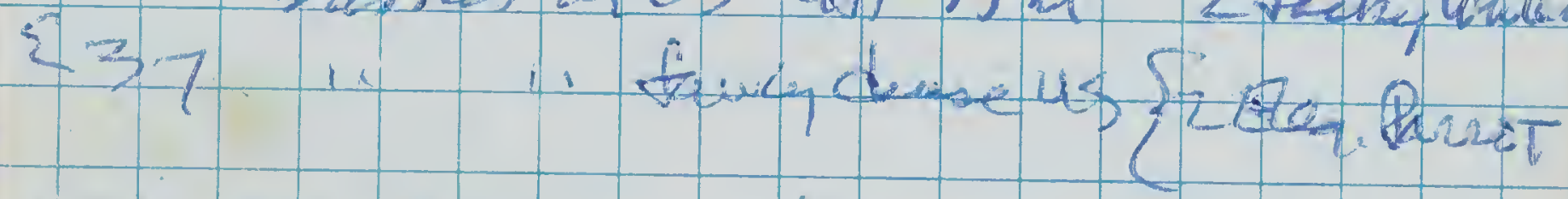

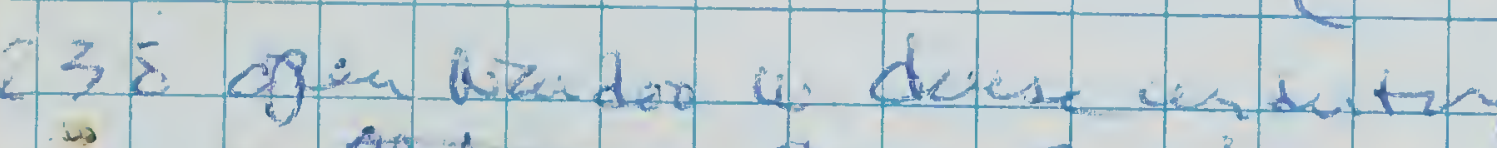

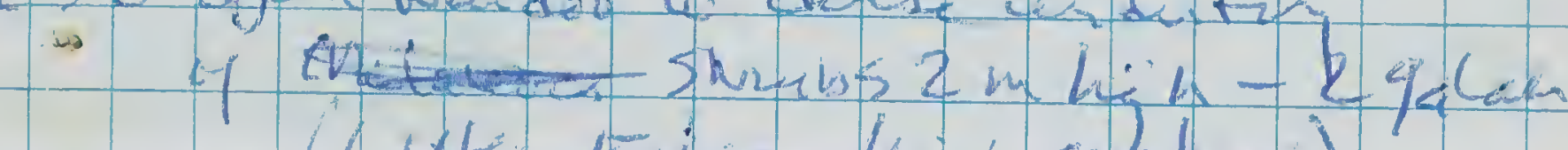
$2 \frac{1}{3} 4 \quad$ is

210 vagen Wraber 12-15n luzh disita olich lis

$$
\text { (Cypseran losm } N)
$$

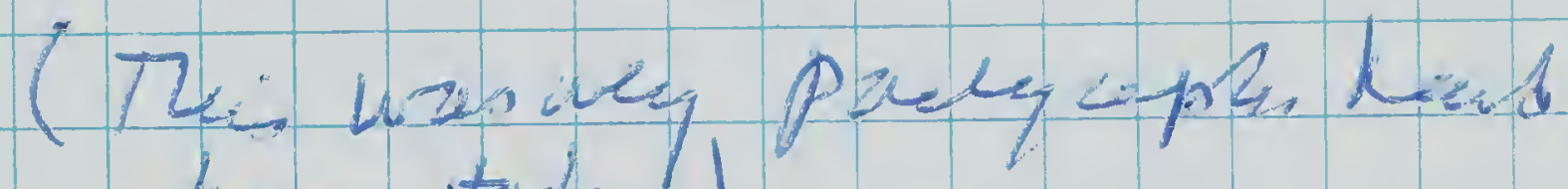
bere testy

busk ot lan IX:10

Red thwend Malke-E. erythrovema White tiagrant qrawlen- 9. ptenosperma

G. Dritzelli-qrean bow hairs 
Rulato clan, are, sibreja

- 06:15 resabe 2 5mis. Duvidopa

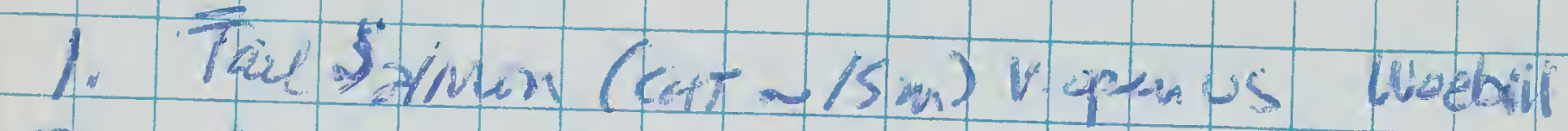

2. " " $" 10$ Jacklw.

3. Wardaric-imopenus Wethill

4. In " neditshups zime.p

a (roflit loums)

5. i1 ' trivindase us of vared skun sp. - zan tak 0

6.1. .. madt sunhs livetsill

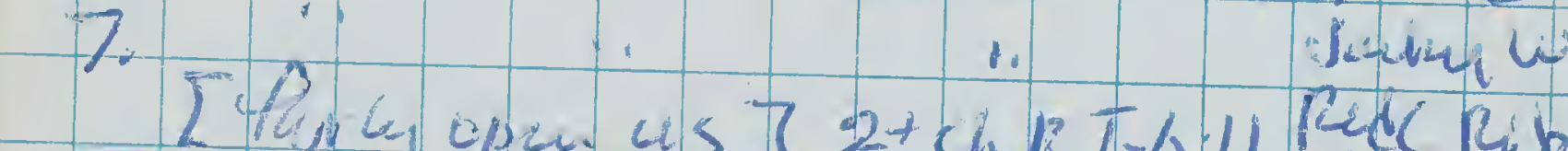
Bat $2+c h k T-11$ Med Rikn

EDCE

8. Wimder $612 \mathrm{mV}$ perins

tein

4 h

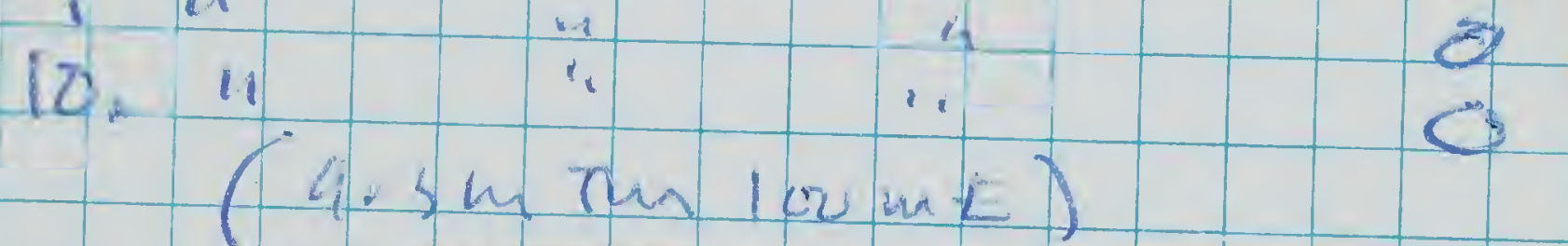

NAT

NT,

Q

Rylar IHt Grshritu llil

uren spp. Paudalite wiredudte

13.1. 'fanjoure us Wreb:lls 14 Is open viñdoem wadt simbs E 17 Waudo-V linte us 8-12 a o

Qiq $\cdots$ i

EDGE

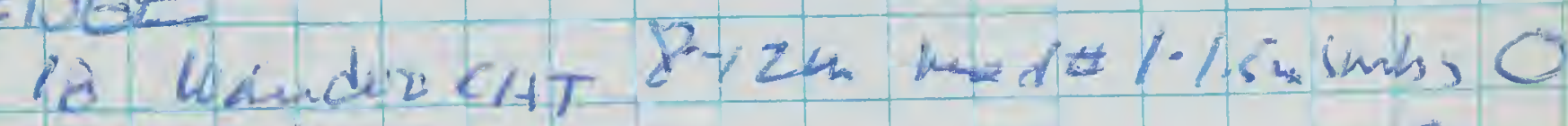
19 in a. $\quad$ aramy un

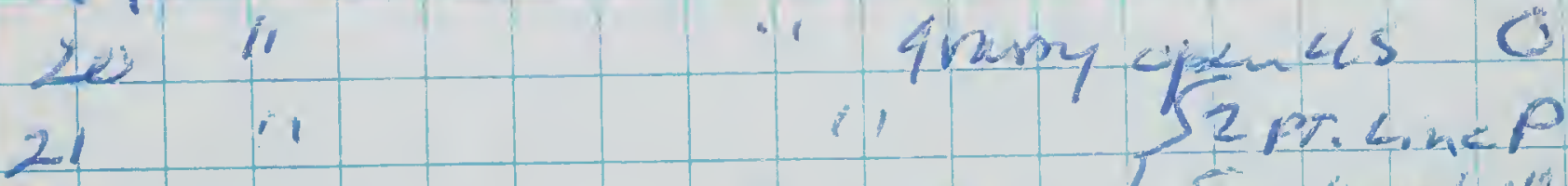

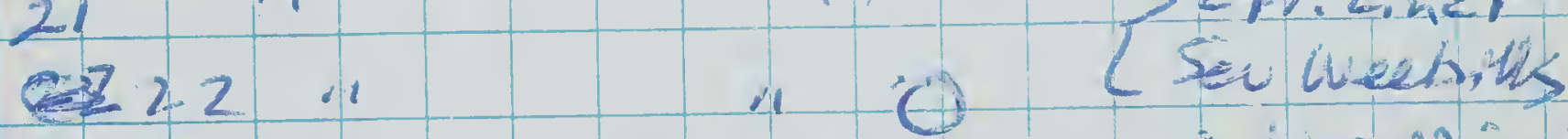
23 i $\quad$ ing rop stytminer

LNT

24 Wha dei/miele CAT 8-12 Sartand sintos

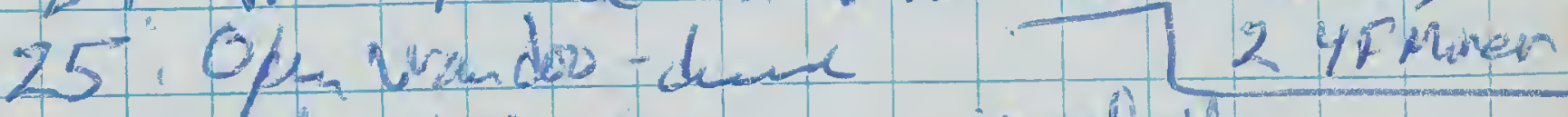
patbeysiumba - weebila

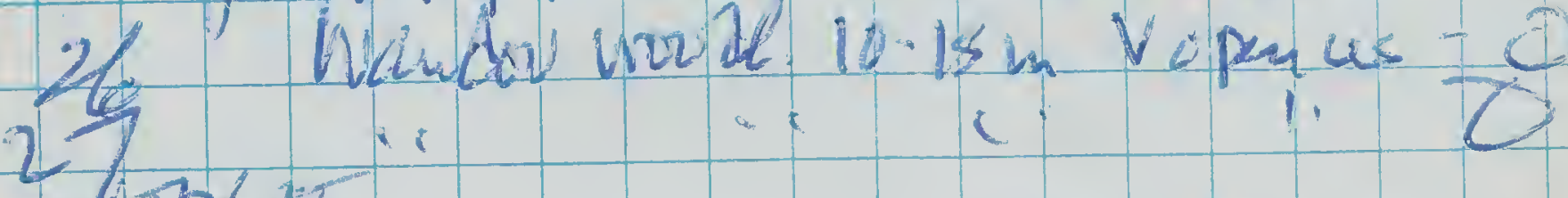




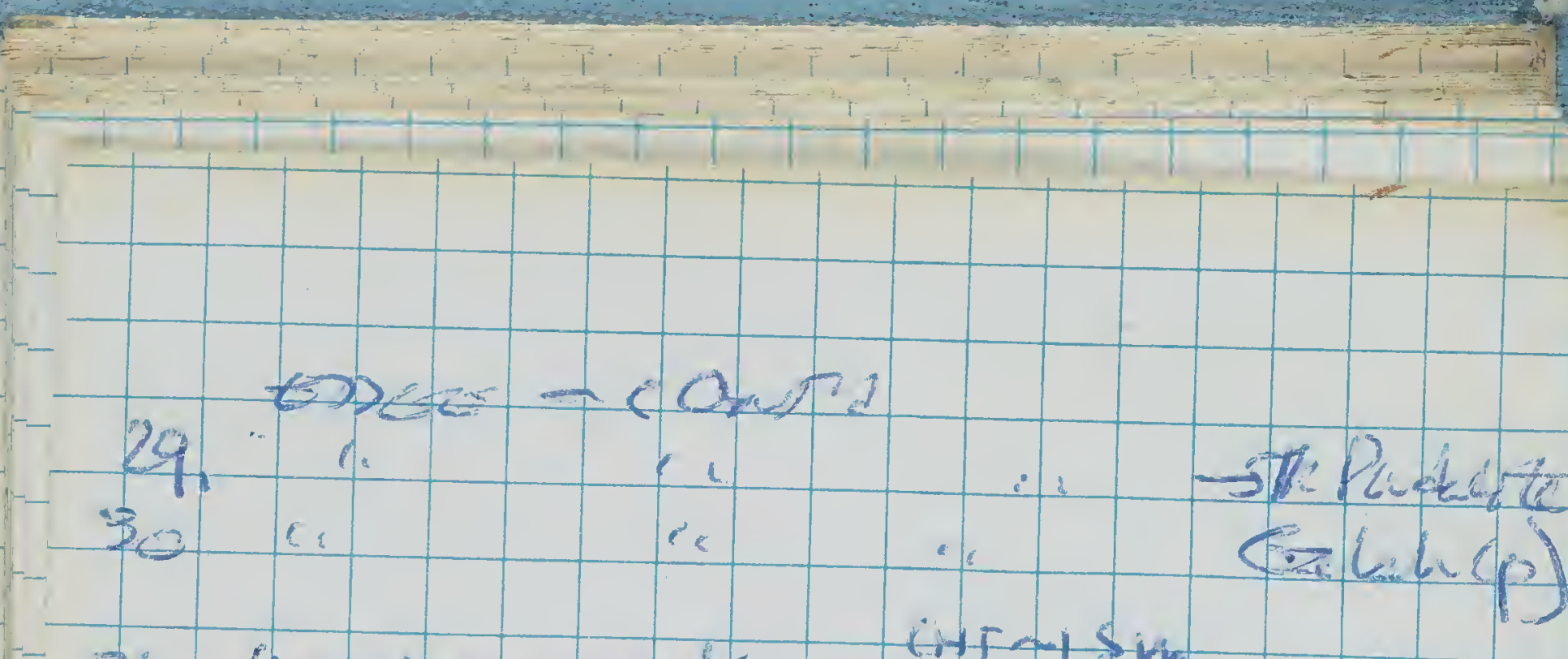

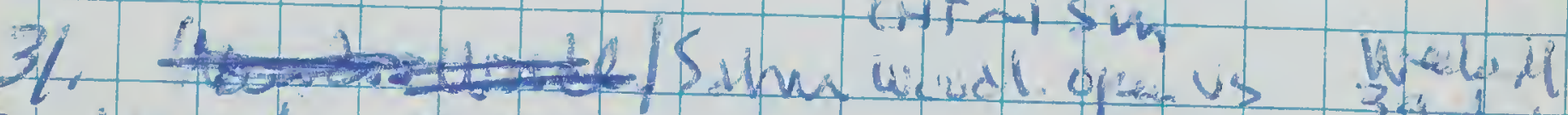

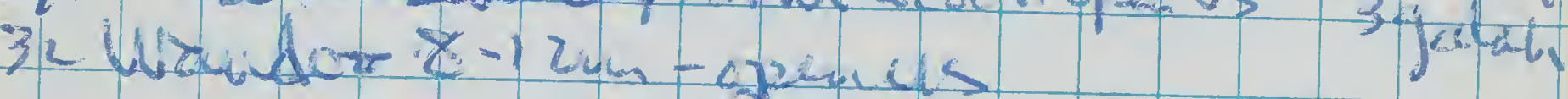
Sir Puntite, Weibut

3.3

34.

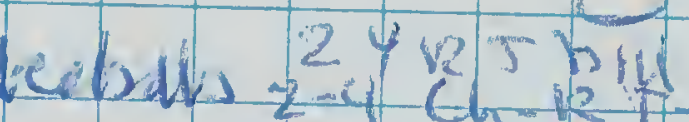
2 рт. Luve P

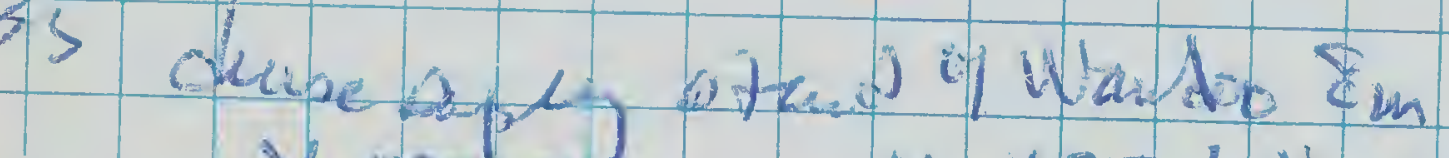

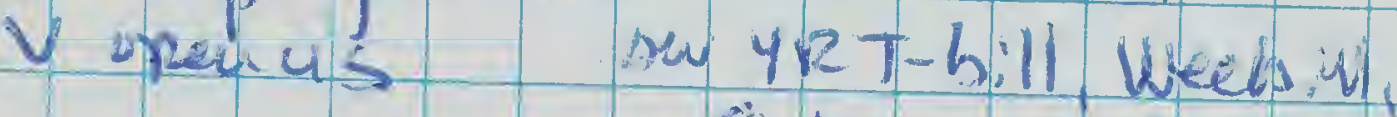
36 Wimdar in in shbraze cucter teriig

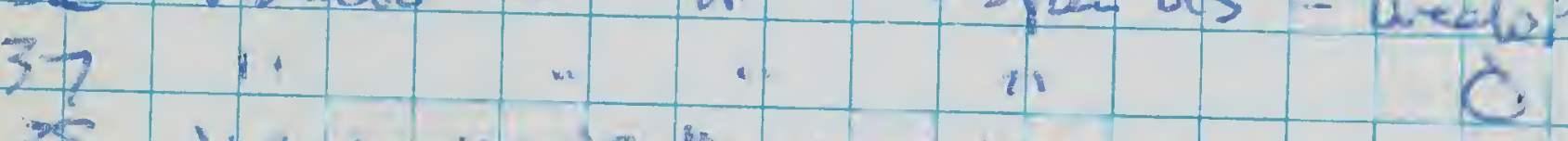

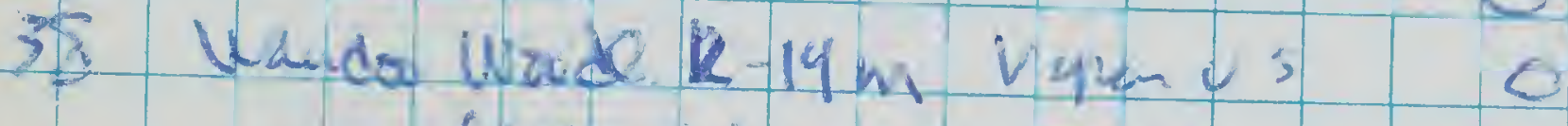
in lut lointo pasa Winere

If Wuader. lom V. urenus 0

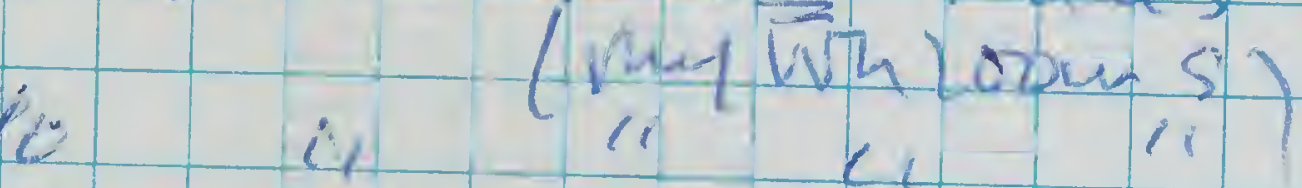

4

42

1.

43

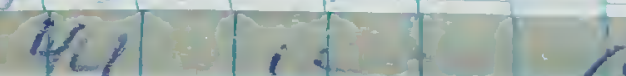

45

4. 1

$47 \div \quad \ldots \quad+1 \quad 0$

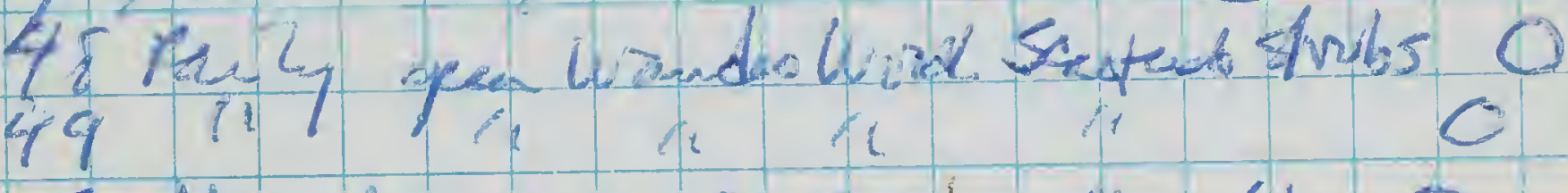

50 Wando $6 x+8-12 m i$ " " 0

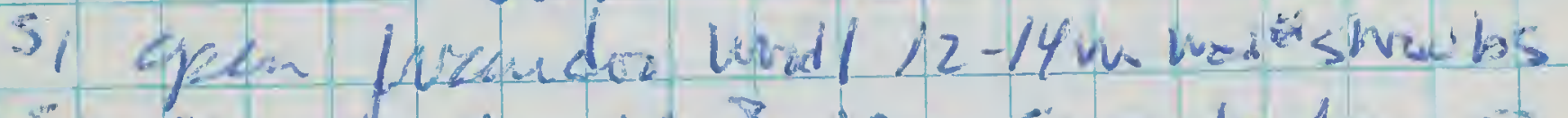

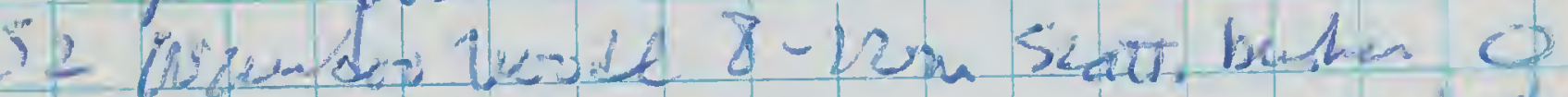

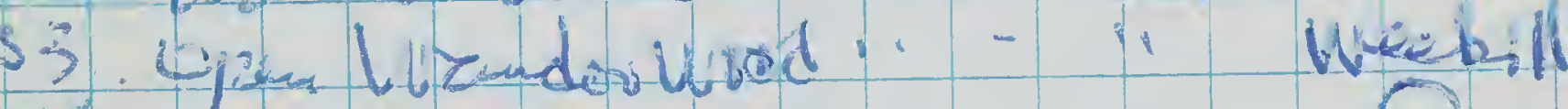
54 a .0

$55 \quad \therefore \quad$ sawhebills

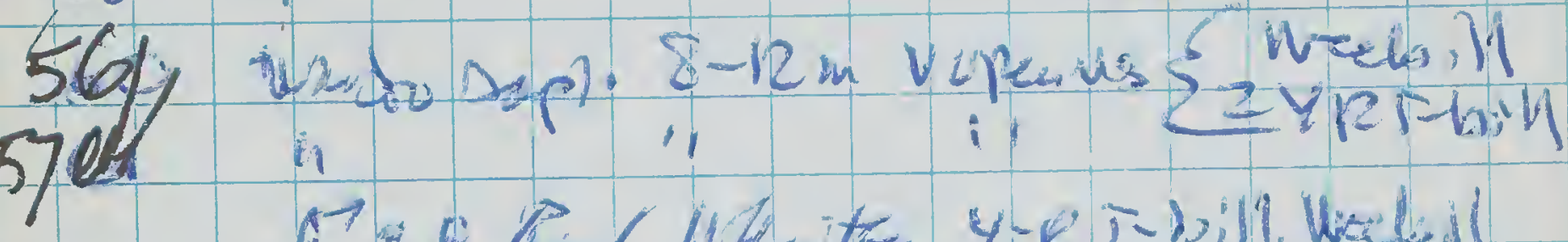

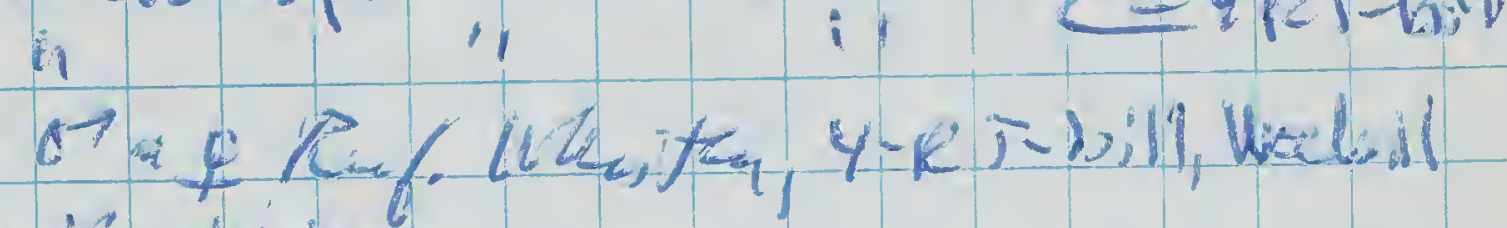

$38 \mathrm{~N}$ i $25: 1+1 A E$

59 cht

wh Wencill 0

$2 D A E$

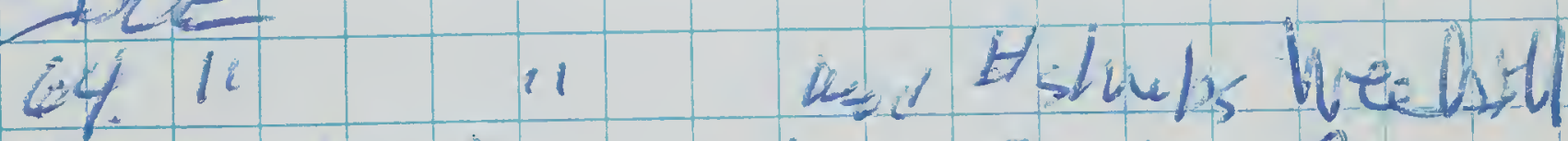

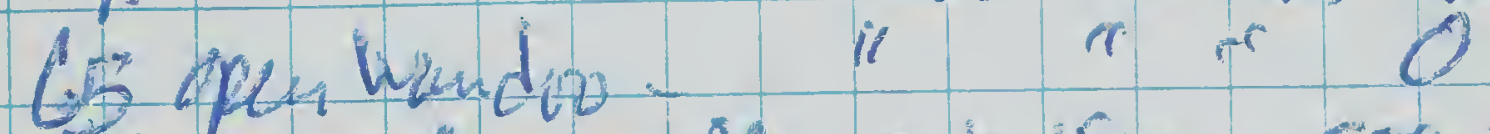

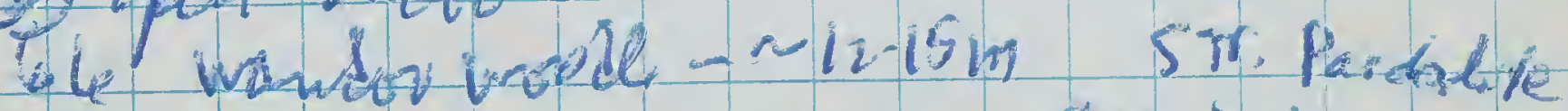

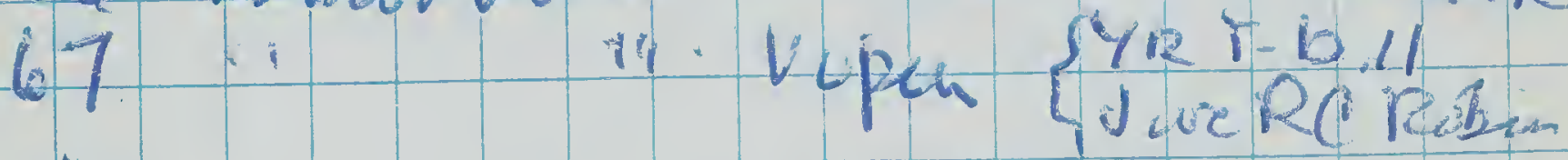
68 i 1 " Dev,YRTbil

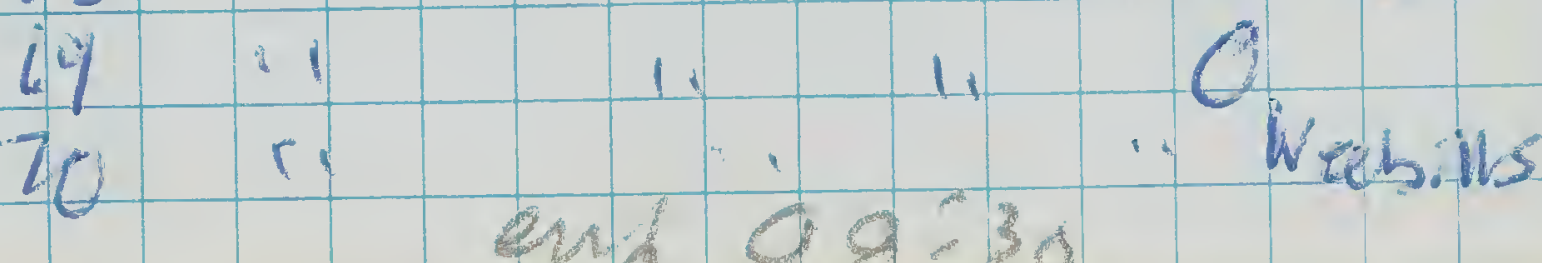




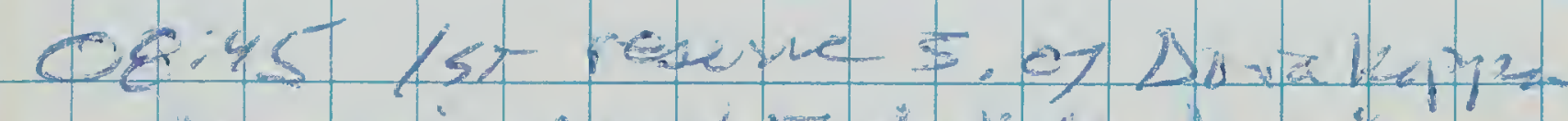

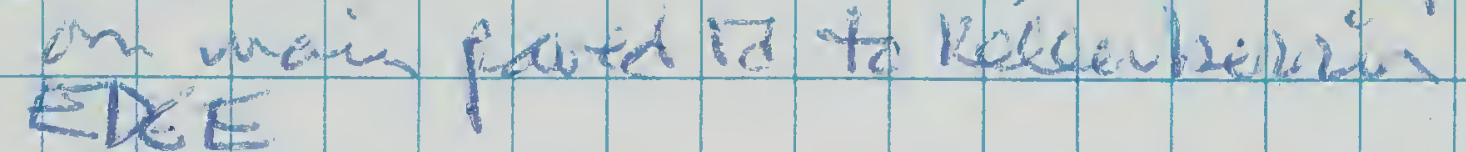

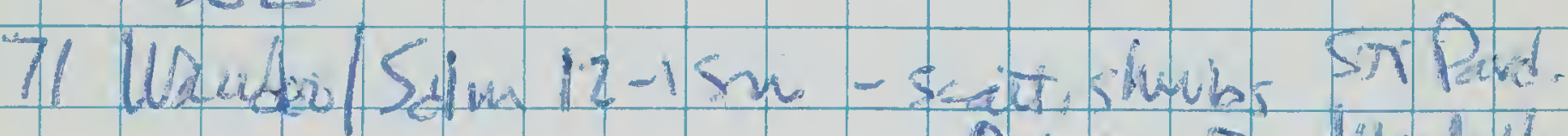
Dring P. Weetsh

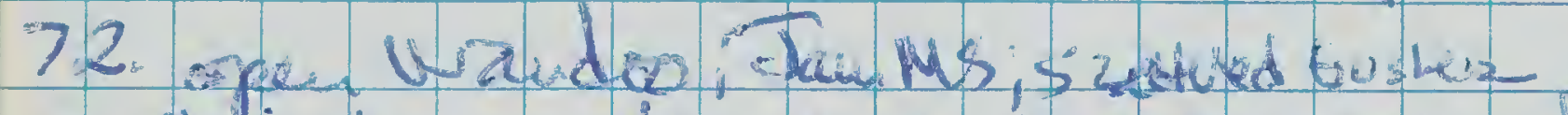

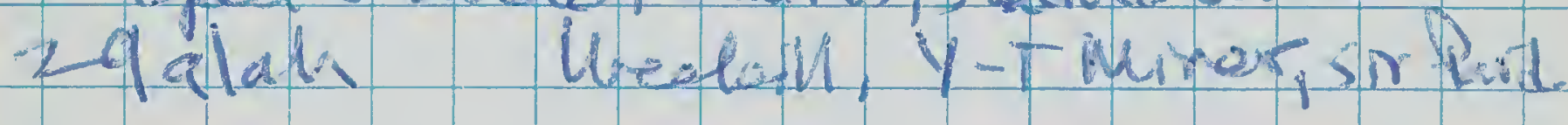

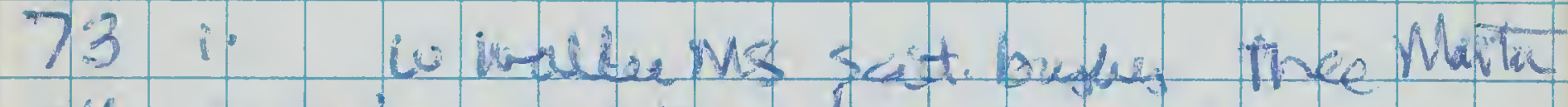

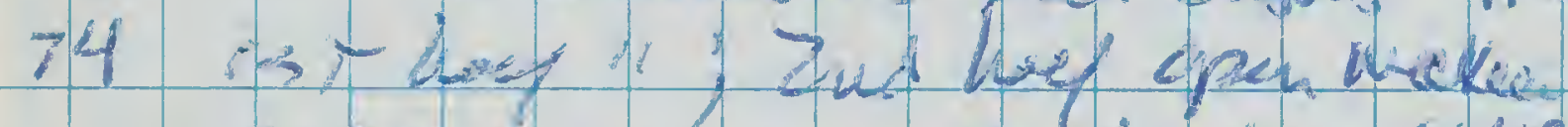

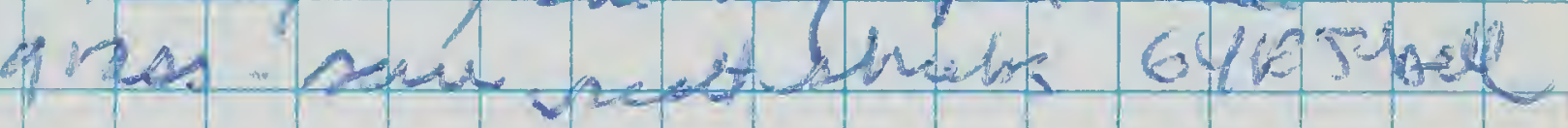

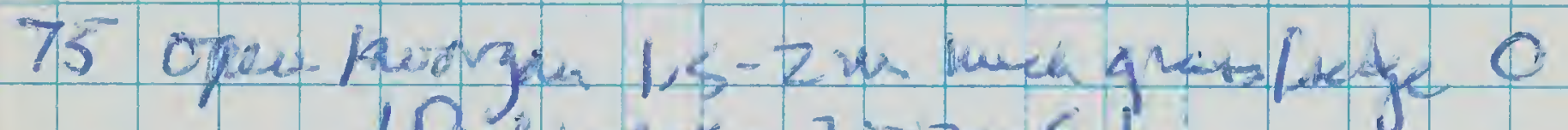
(Dif werker $702 \mathrm{~ms}$ )

- L Tak dims kermain-Cas te $4 \mathrm{~m}$ P. Live P.

$77:$ SINUE

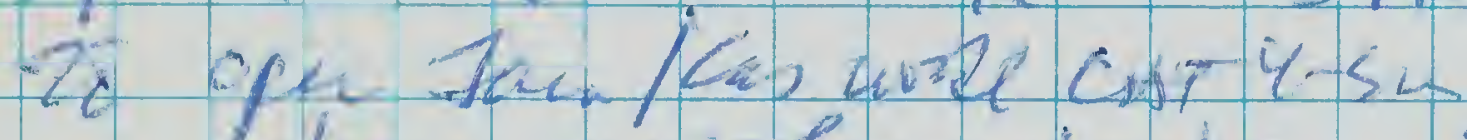

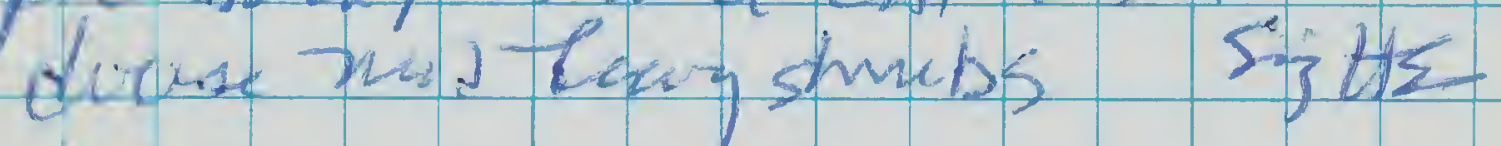

NTEvis on

79 Jom/chat arzh. 4-5m

stext. Jim s in 25

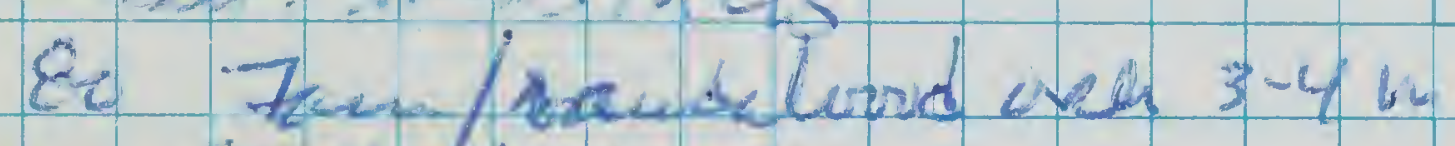

sied lawhos 0

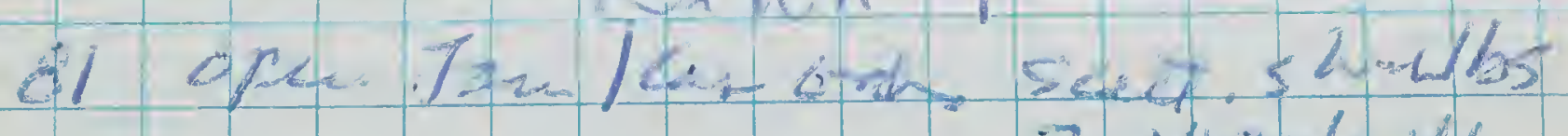
2 edestan

82 open Jaw/band arch ked

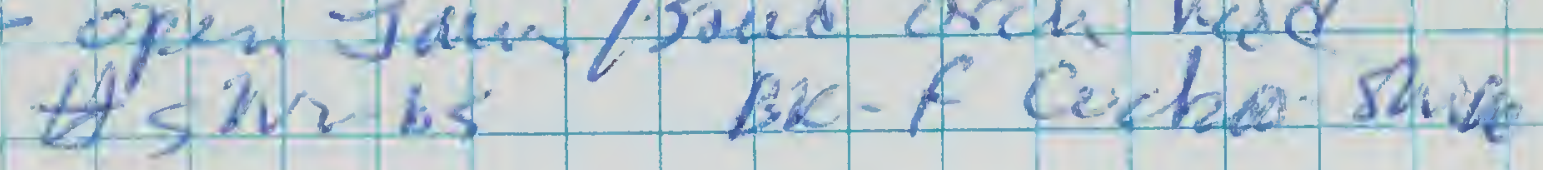

T3 oremlyok wL; Jaums wad 4 huobat - woebils, 2 nugpe

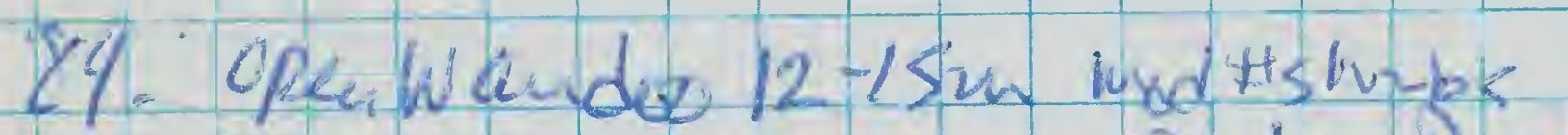
qusinin 2 galah PT. Jax P. vechill

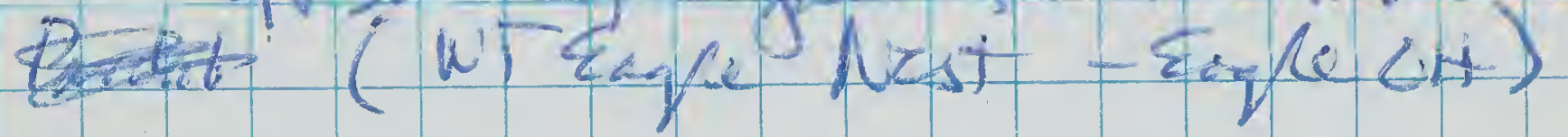

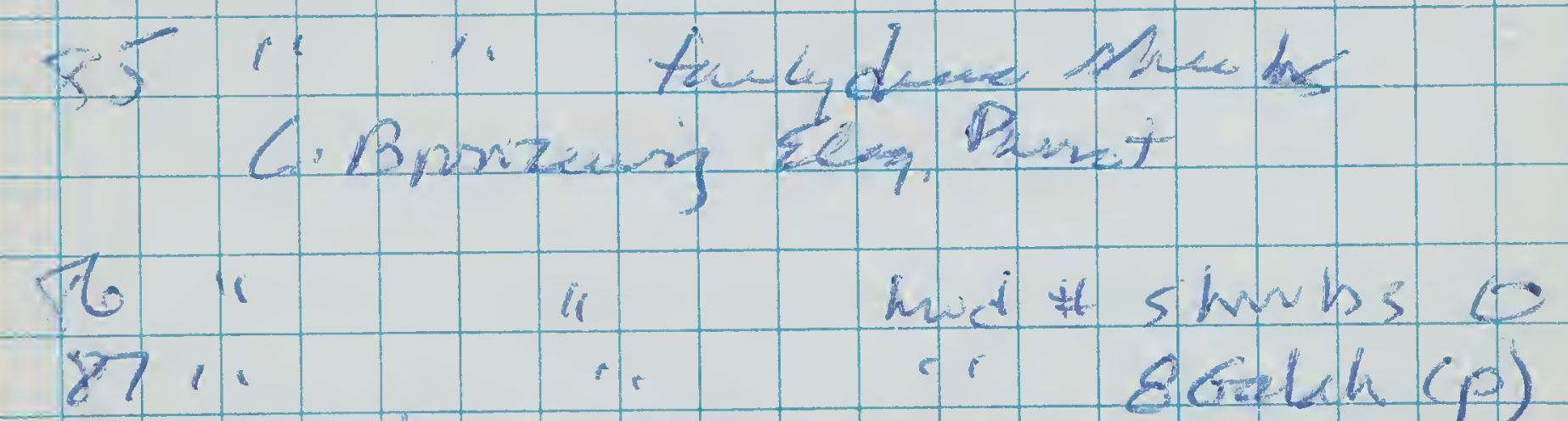

88 Wander Wandl Low nad shous - 0

89 ". "Prlinep

$901 . \quad \cdots \quad$ Sev 4 FMMner

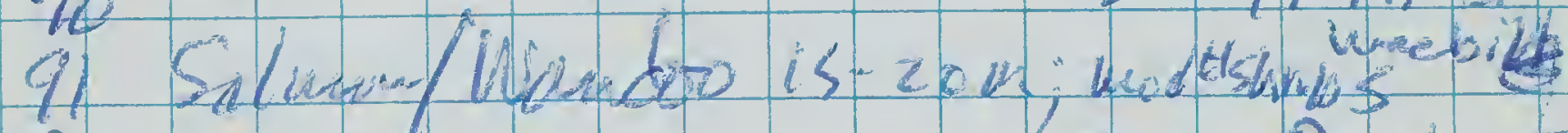

in i. \& pandecte

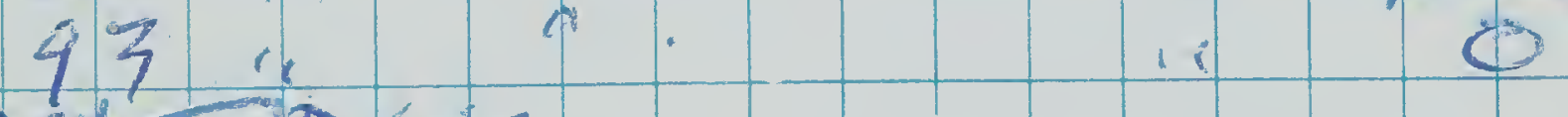

$\rightarrow-4$

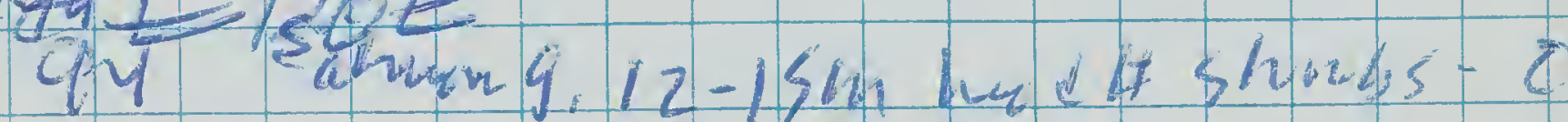

$99411 \quad$ in a prater 
$-206-4 n+d$

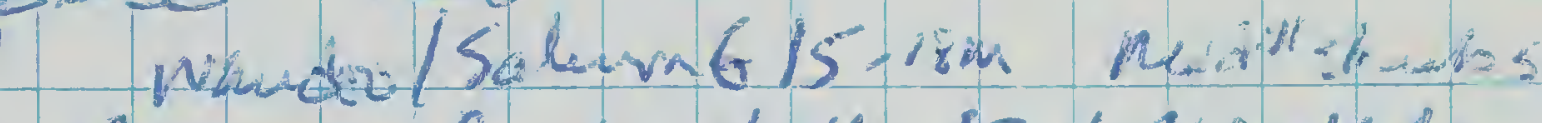

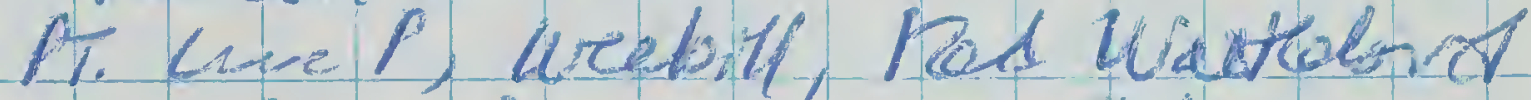

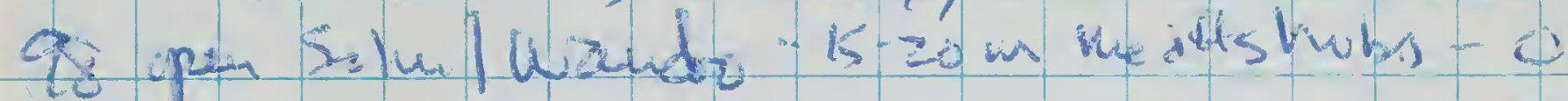
a'a

lose

2PT L, nc P

Finisn /Croatan

12. Csin contage cit 10:40 $19,636-2$

Gas Mrthme $810=177699$

E: Yorkratine

hr. G S00-Re, whistler vest

su. Patzh 1 Casuasiisa. ( $-3 \mathrm{~m}$ tace) un area 1 Vunda - nes on Wi side

\section{Rundwr Red/Mctal left}

Work Plan for Kellerbersin 1-3 Nov

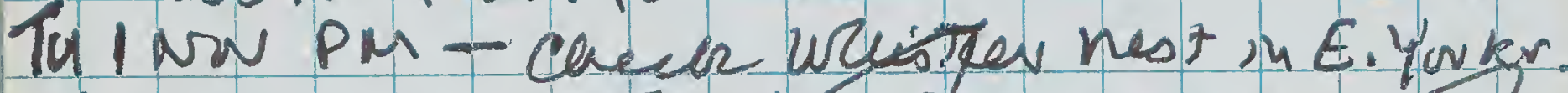
WNON.AM - Si ekge DurokoppuE covridor fr. Dyt.E to Kodjkodjug, No. end kodj Kadjiro

- PM - st edge of Weeds, surall private -patar at SE Corver veeds

Th 3 Nov/AM - Corfidor/aien mai /rd. fr. Dur. E. throngh Pumplous to No-Nave and across to Mission Rd. (pm duive to S. Corss)

F.4 Nov - Yeleowdin area

5 NN (ANA)

com-dorre to Reederberin 
Tu - Net at E. Yorkrakive Band RuFwe.

wed - Kodj Kodjin corvidor Am N. Kodi codin

S. edqe E. Durakoppin

PM SE edqe weeds (drive to S. Cross)

Thurs Yeecoudene

Fri (ton) "

\section{Sat (AM)}

(PM - drive to Keelerbenion)

1 NN88

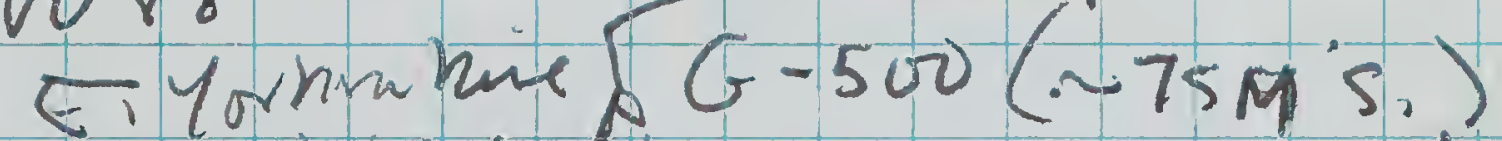

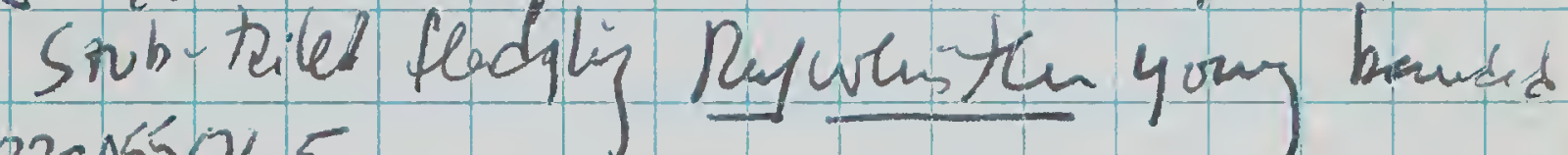
$032-055065$

Yeinow somedrunabore eyes

gape irow throut

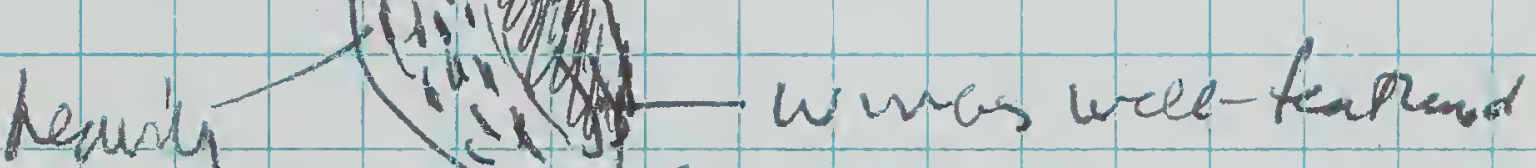

streated

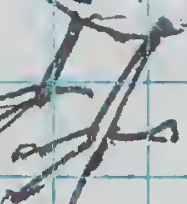

Lessentially bi hil

qives oceasional short "pip'"caes

on quin "Whit!" and

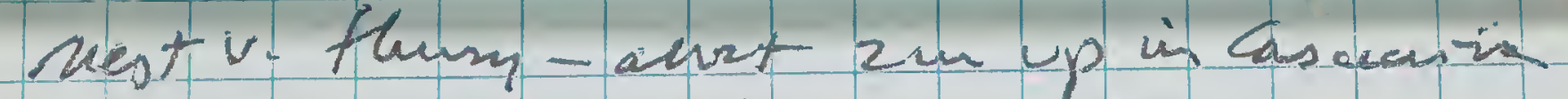

at wisite of a $30 \mathrm{~m} \times 3 \mathrm{~cm}$ ypen

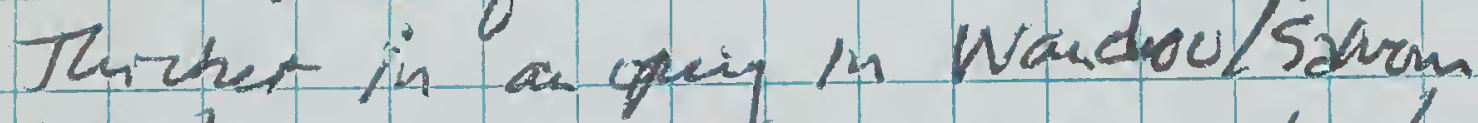

unds- no orku Casvana meky lat

clumps of cteen sheros y mallee nrby.

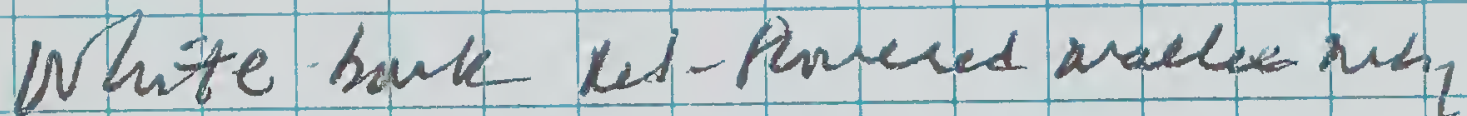

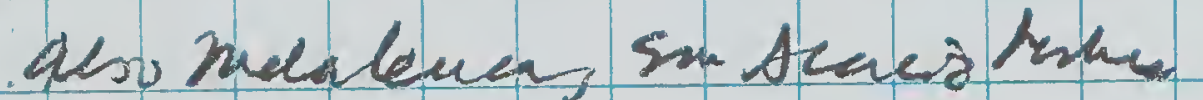

2 NoV INTERIOR Aurnopin Lost 5. edge ur. SE SW comu-N. Nay kagaw incuser lin

Obios clam callu, wild katered 3Pr.Luc

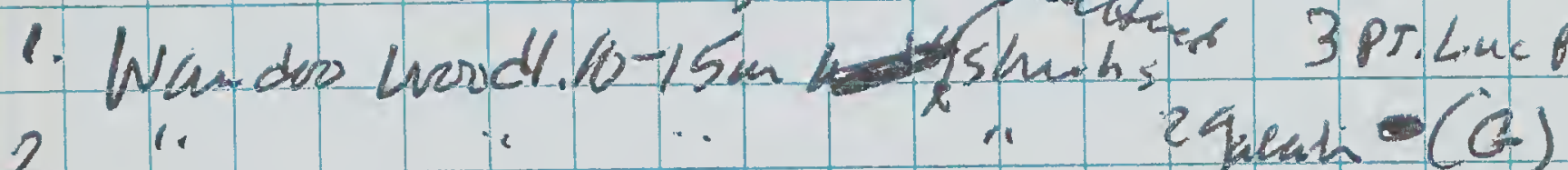
2 (B-2000 hare)

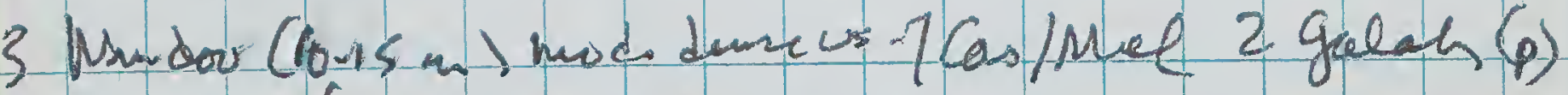
(aut west here)

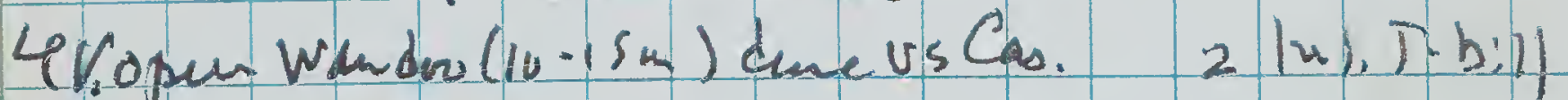
5 open wandos (10-15a) ; sant. sumbs or o 6 ". 11 "ar hother. 7 wandou wod $(12-18 \mathrm{~m})$ scattshr-o PT: Lime P, Gulah

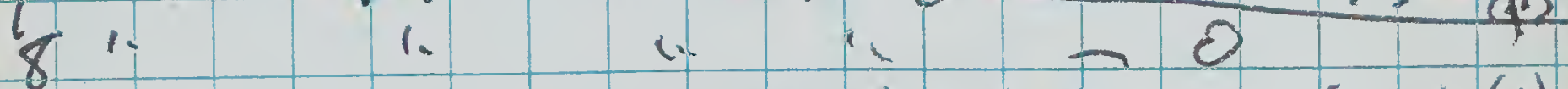
q. $\quad$ " imod stoubs 2 Gowh (p) q (Rulwintler o? $150 \mathrm{~m}$ N.)

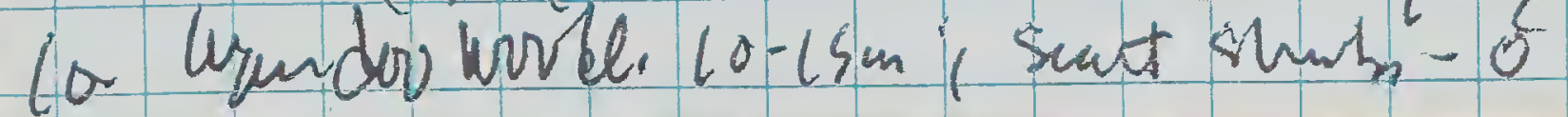




\section{2 ner cut d Dustroppon Eust}

Gorsth 18t III Rym 111

C. Bueubird I

Tum N. los m shm of road

11. Goundor hood. 10-15m;seat, sumbs-

Ref. Whit ten (save as hoo ), 2 Weebill

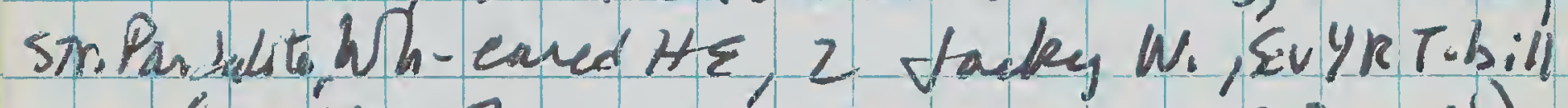
Consh. Thu $\sim 150 \mathrm{~m} N$, Beelbias $200 \mathrm{~m} N)$ (Aumeast)

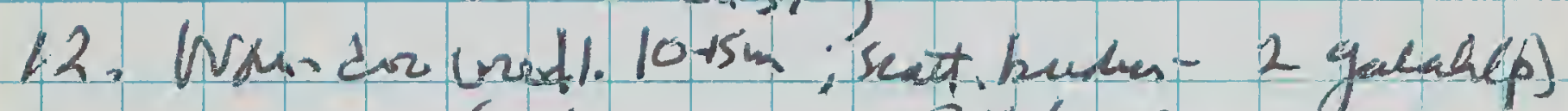
$13=-4$ insdeburas $=0$

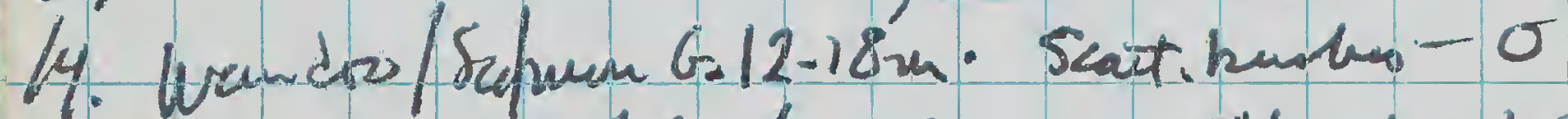

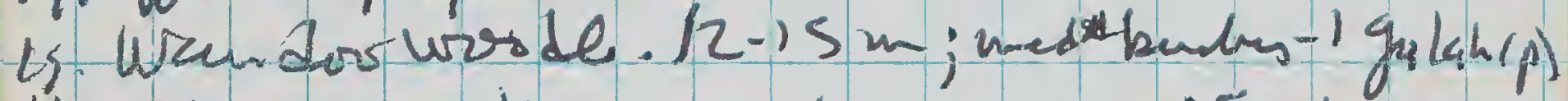

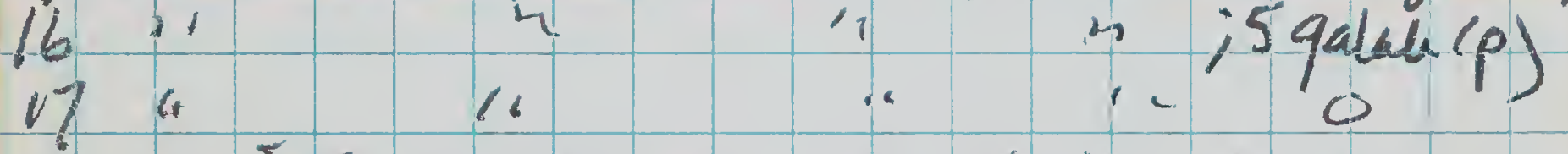

This is $3 \mathrm{sen}$ shut of Ni-S kaypum L moser alng 2000 line - teinn N here? 12. Wiandro 10-15m: Girly demse US Oencanina

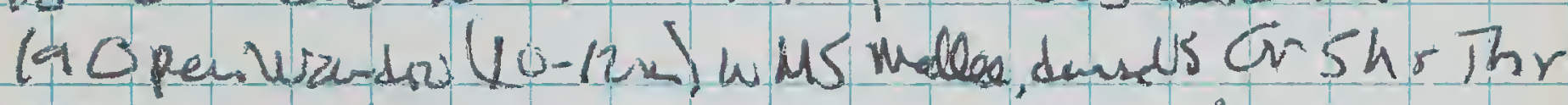

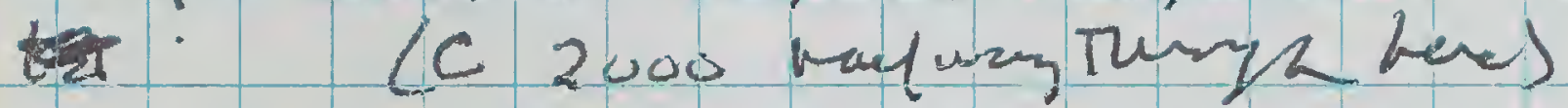
Turn W. baeks towand Id.

20. deuse mel / caybath $(2-2.5 \mathrm{~m}) 0$

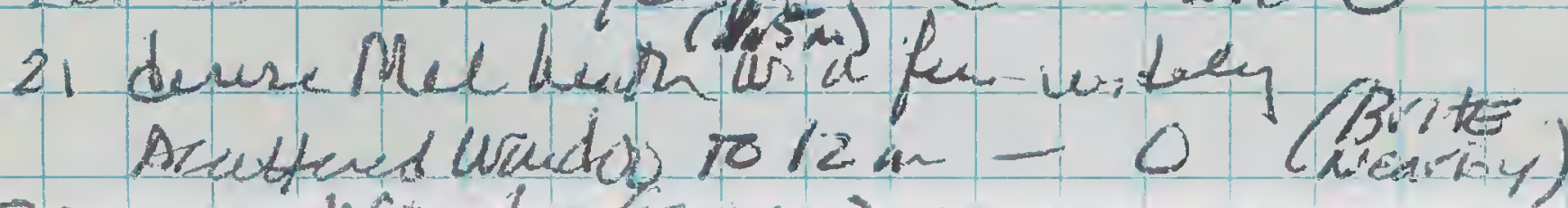

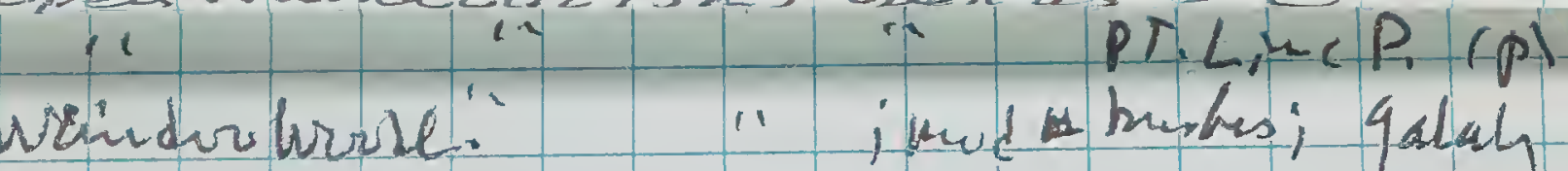

25 Waidw lorr 4.4 Counshrt of rd-start zaln Edque rema?

\section{EDGE}

26. Wando waid 12-15mi openus - 0 $22 \ldots \ldots$ isents shubs-0

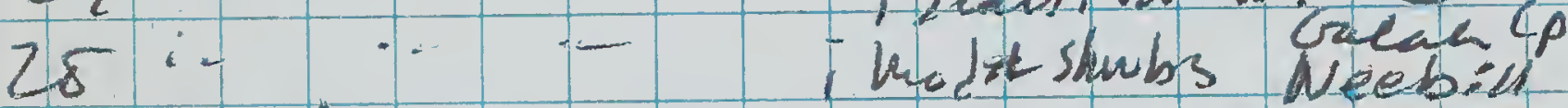

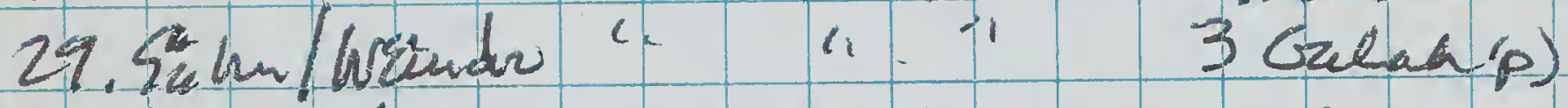

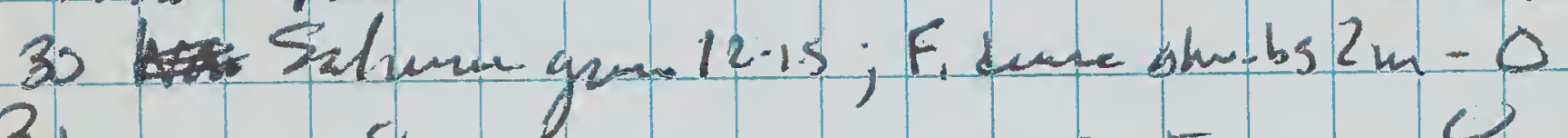
3. 32.

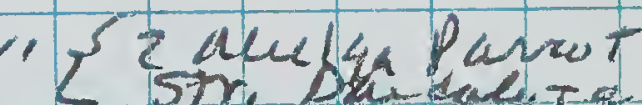
33.F. Deque Cas/mal/et, neath (1.5-2.5m) o

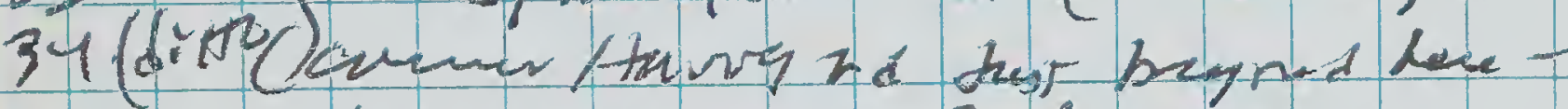
tren cast aengedge of resums 35

32

$31 \cdots+\sin 6 t^{2}$

38 a sati wandrotorim

40 finch bure Cas other shos ro $2 \mathrm{~m} 0$ (N-S track to can hew) If $:$ i 120 [2000 line (niduray]

42 oper wando $(10.12 n)$ w. danse us of cas, shibs 2 ph him Pardalok 
43 V-open lasandos lo-12u; opearms uollee, duse us Casere. Eleq.Parrot, Sinc. HE

$44 \mathrm{C}, 2 \cdots$ Pr Linc P. 2 Bi-hdHE

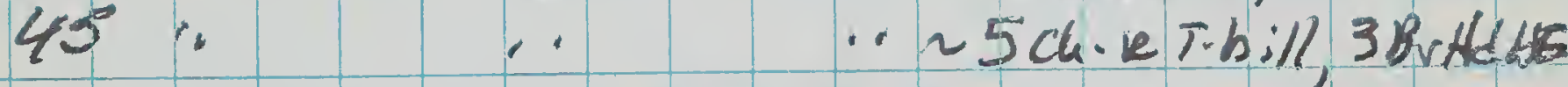
Imain N.S Track bees

46 Son Babblers (qushrthe loom N)

47. PrHE, Weellill

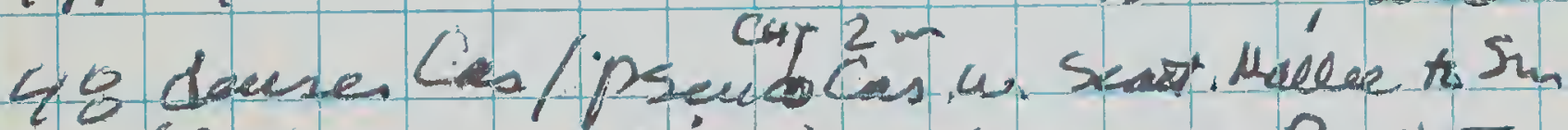

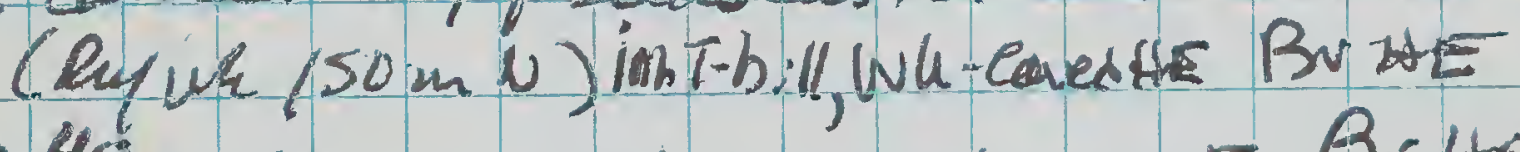
49 i. $49, \ldots$ - BrHE

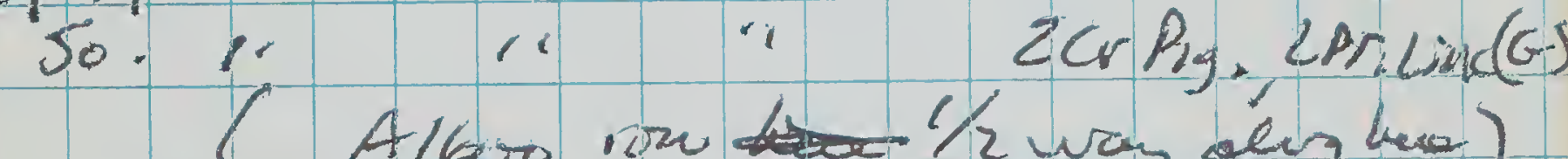
s. dane Ceser. Thinhts 1.5-2an qrshitar 52 53

54 "L 0 . (Alfor hine 1/2 way)

(1) 0

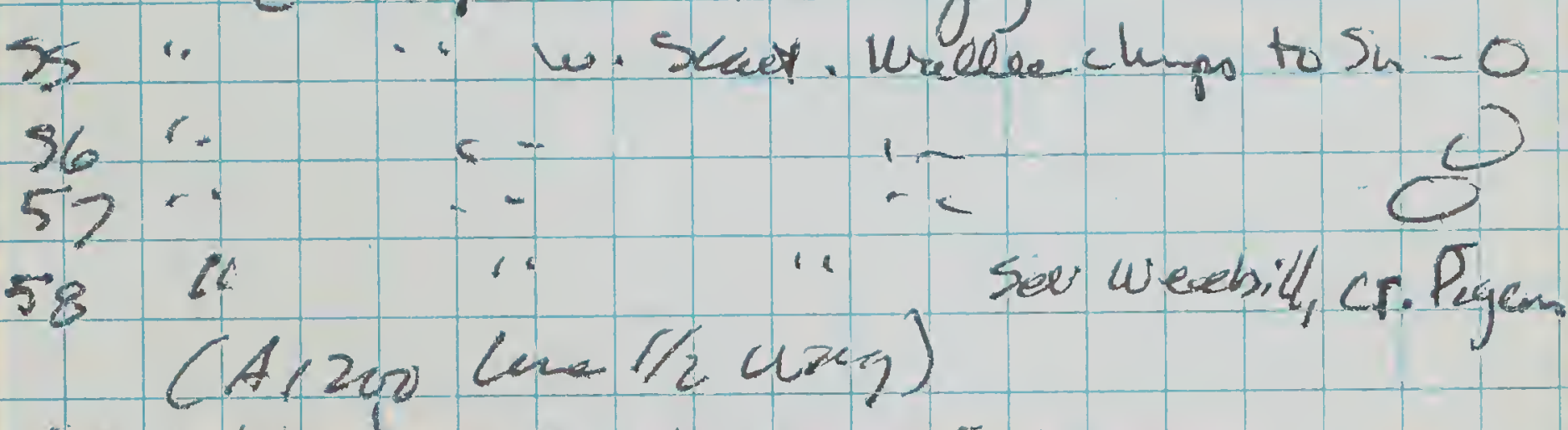

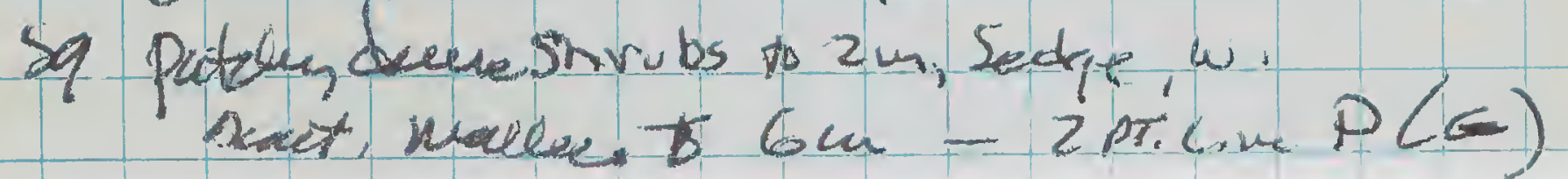

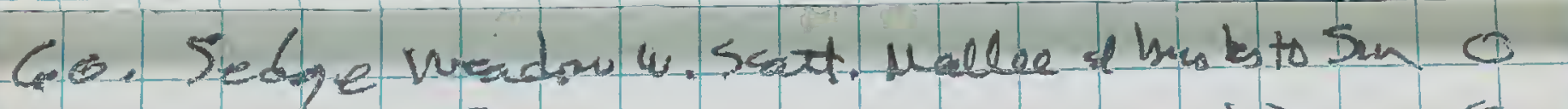

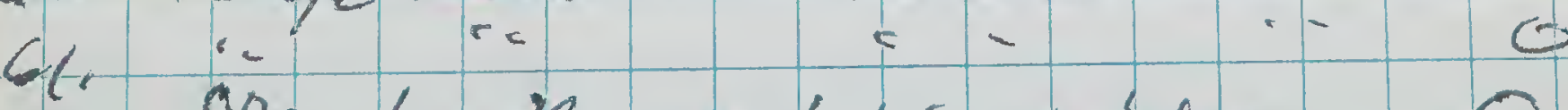
C2 Pen low Kwajanl-1.5a a. Sebge - O (A-1000 bayevon thong)

63

6034

$$
\text { (A-qor baum rurzh) }
$$

$64 \% \quad \ldots \quad$ CrPTilime $P(P)$ INTERLOR

I cut N an 800 'Ro hima $\checkmark$ stath at 4980

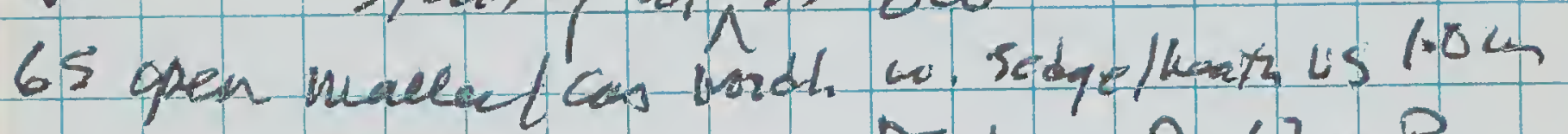
P. Linc P, tlea. P $66 "$ "itairlydeneshub is $1,5 \cdot 2$ m 0 67 (1, B-800 have

$68 \ldots$ Weabills Co open Wandoro $12-15 \mathrm{~m}$; fairly demen Meletz US. ips Lund 70 a " " "swiweebills (9rs m in $100 \mathrm{~m} \mathrm{W)}$

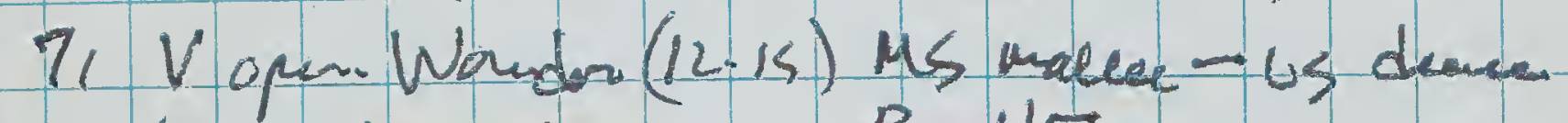
mel, an, ete. BrHE (C. goo here)

72 73 wandoo Urribl 12 - $15 \mathrm{~m}$ i sat. shitos 4 Galuh, seo weebill, Ped Buten? Cheatuiring up in in un wandor (Yim hive-blaik cup, qu. bodq) 
74. Nadv wrode $\sim$ ism; millen Ms, vad 4 smulos Us Sn: Pudolate 26 PT.Ginap

25 open wandro: burevs Cea $2 m$ -

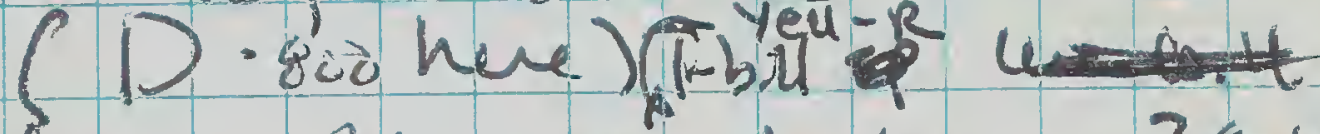

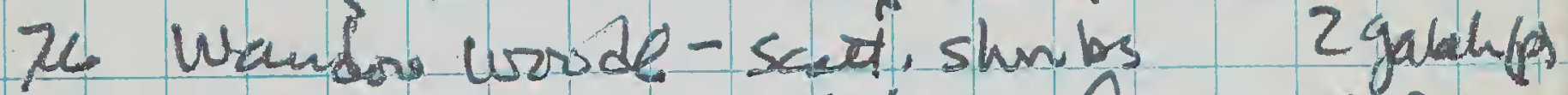
77 " knd beme Cas us-CrPigen Zs openurandso 12-isu sut. Cas $\{2+4-R T \cdot$ bill 29 usunder wond $15-184$; Scat suniss. Pardate

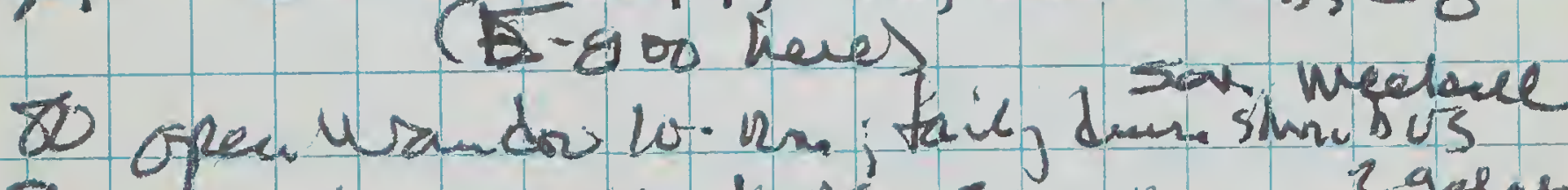

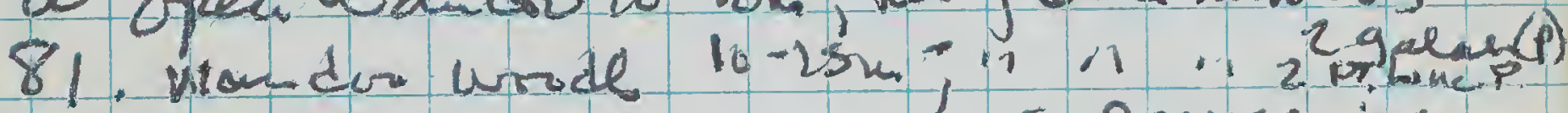
Carshrt the 100-150mw) a Bronzewing

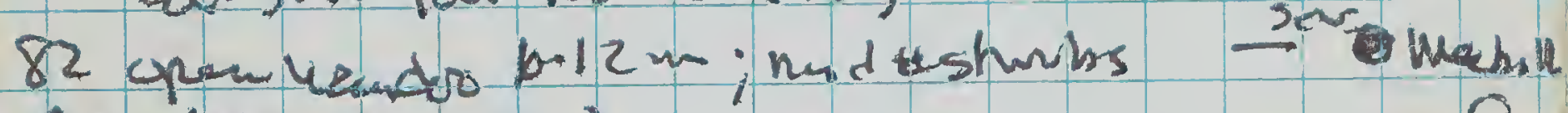
834 to 7 .

\section{(F \& ha hav)}

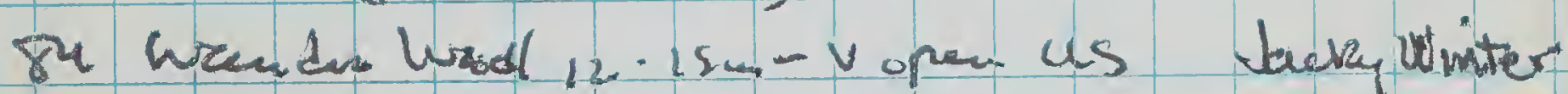
851.4 10-12 ans scatered surubs wealsh's 86 "sen $n$;und shmas wealarts 87 Mralle 3 -5m; dume us oflas,eti. o

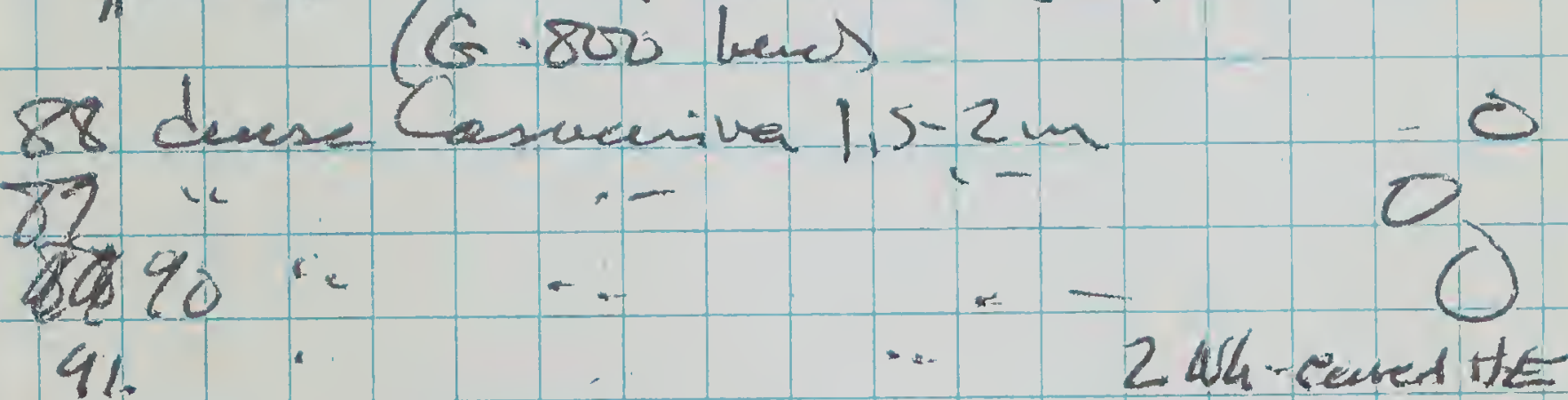
(4 $800 \mathrm{hec})$

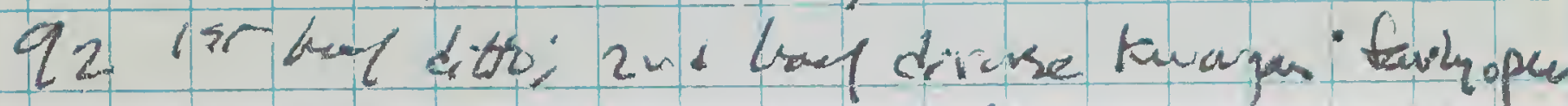

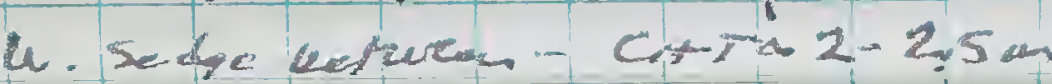

q3 lak last $1 / 2$ 42 0

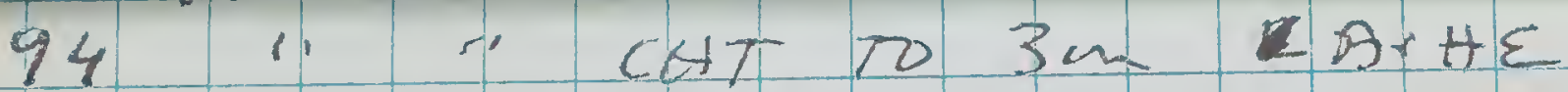
[E-a Trade her-tarnw.]

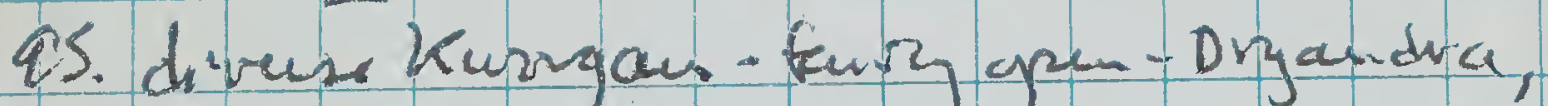
qrewillen, Can, ere.Cittri2m 4 Birtse

te $x$. : -

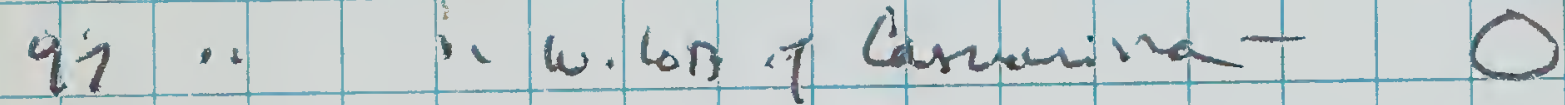

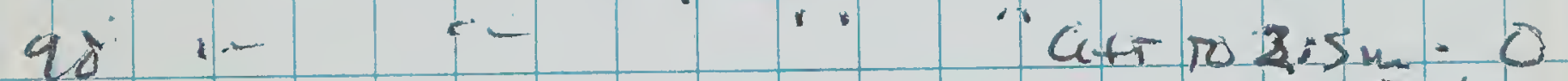
29 " la a conple of wandor 2 Wharer HE

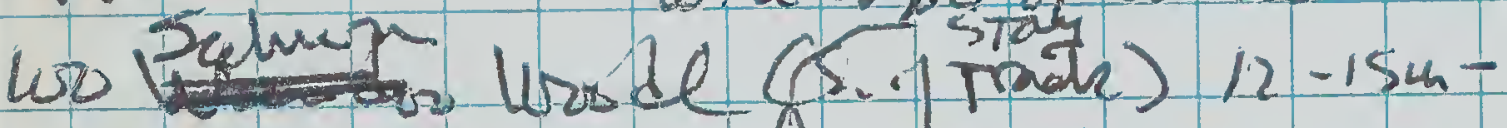
scatad hushes arabill, 4 balah ( $p)$ (0) .10 V.men is $=0$ $102 \ldots-0-0$

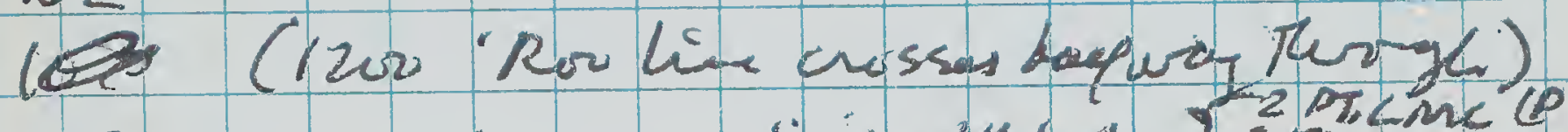

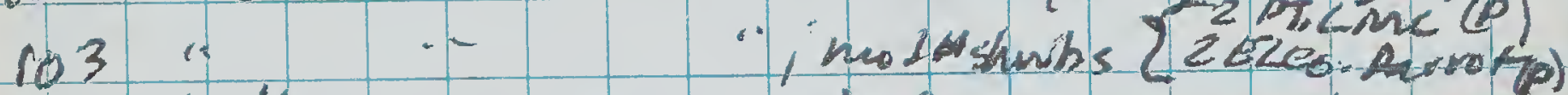
Hoge un wallard of qraq $k$, wave curgabin V. Mave Msaud heata us (orshr Ther $75 \mathrm{~m} \mathrm{~N}$ )

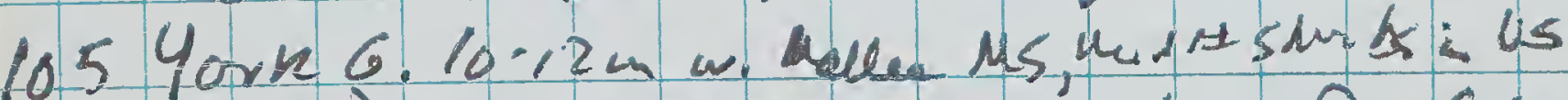

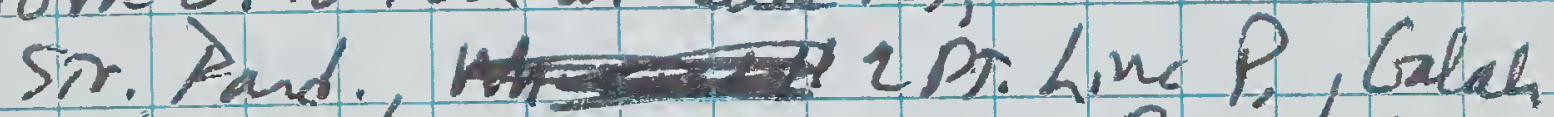
(Re/ whiten Nloom 5.) Raves (p)

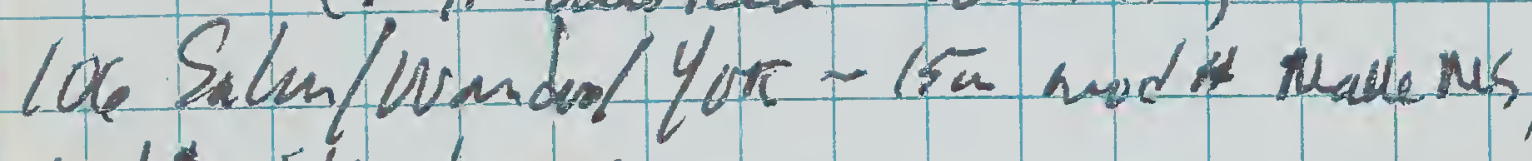
med simbs us -

$$
4-1400 \text { here }
$$

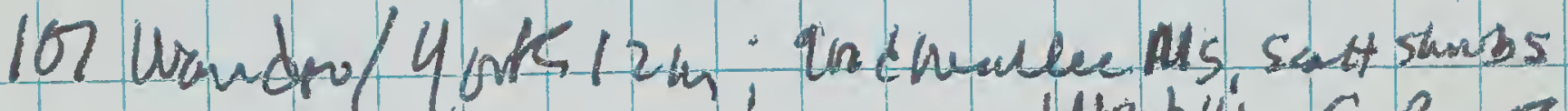
in us Webill, Cisorizan cut N. Sliahtly to road

108 cune Camaring 9 raw Thibes $2 \mathrm{~m} 0$

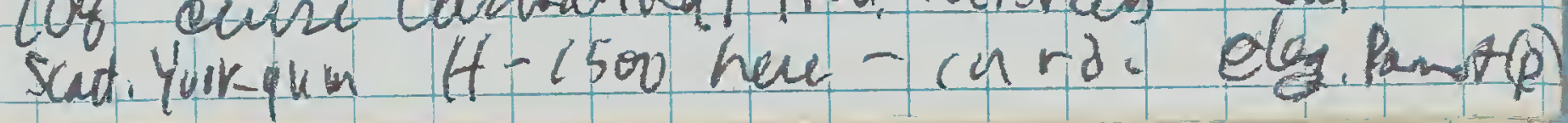


13 i a o i 0

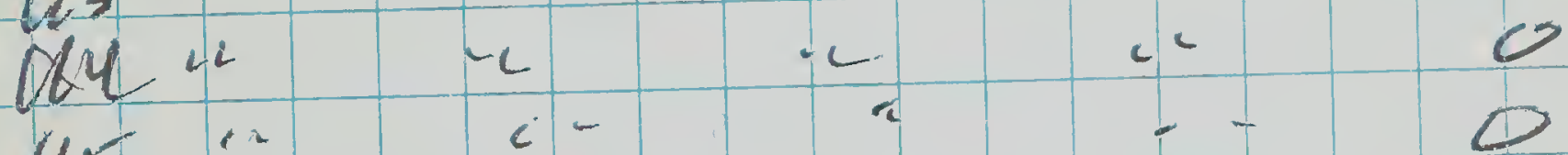
$115 \quad(F-1600$ here $)$

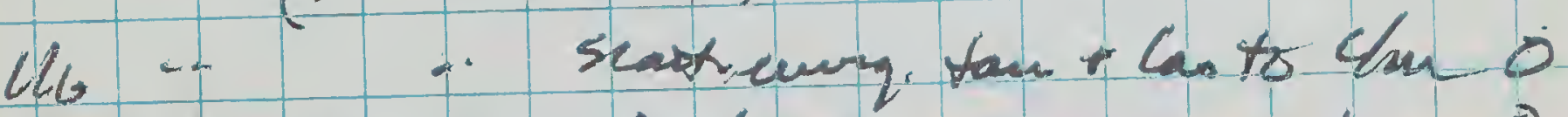

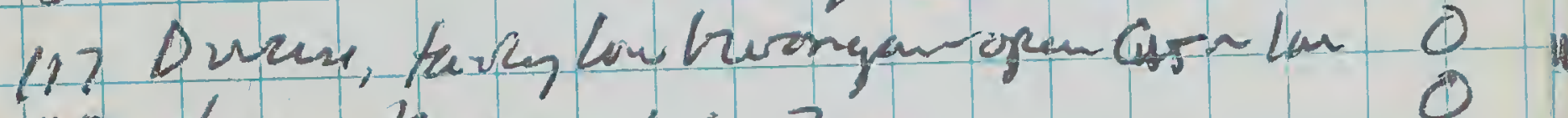
18 tacen kiongen/.5-2m

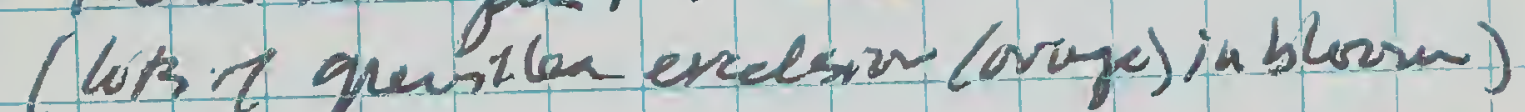

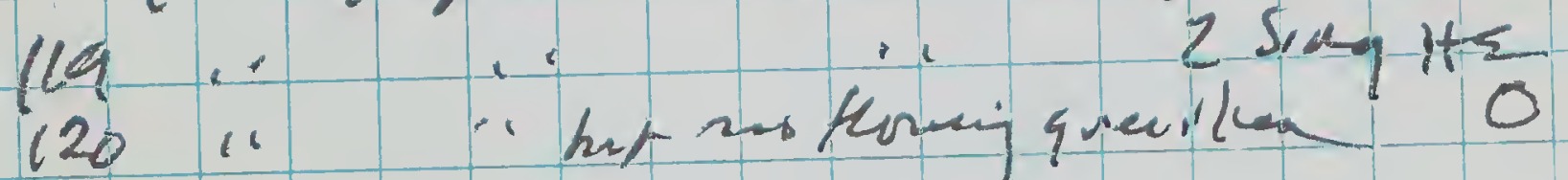

Donous

Gestowish Juve Sinq HE?

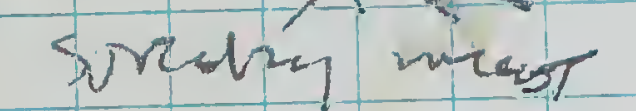
K

$121 \ldots 2$ Juvesint?

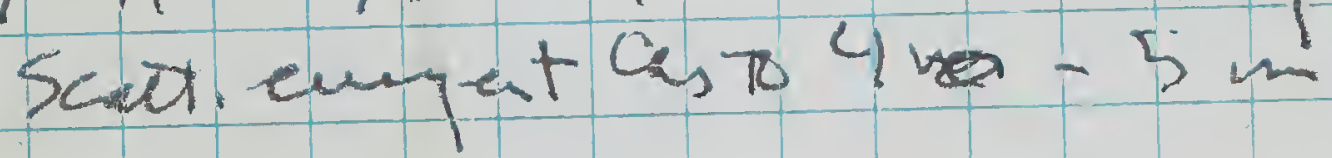

\section{2}

(2) 13 is a r a Sique Brits $126 \%$ zotace curgents Zuh Tibi4 $422 \cdots \cdots$

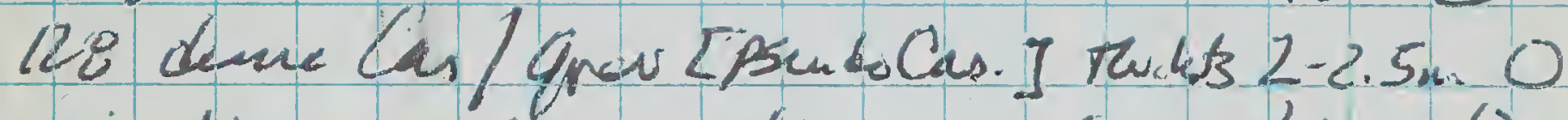

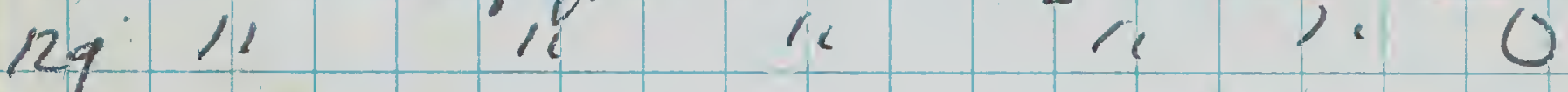
$130 \ldots \ldots$ Wa-eared HE (3) A is seathed Malle to $5 \mathrm{~m}-0$ woinabst zoom N. of Harvegrdtevalu to do in teur trasect bach to car)

132 Wandor hrobl 10 12 m j und da shums (wel, Ch) in us mallems; 133 C. Bunzeunta car $(00 \mathrm{~m} \mathrm{sw})$

oq:5o stop 10:05 N. Edqe Kodj Kodjin (Maley Ral 
LDEE KoDJ KoDjin-N. Side Maleyrd 135 gean wandos Ism; seat shrb us Wriakardis 136 o

131 Malloe (3m) wod darsity surabs us 0

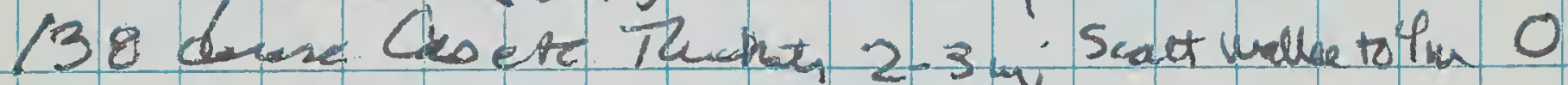
isa open Mader (3- Yu) w dhe hel us - 0 140 open wardoo $(10.12 \mathrm{~m})$; Malle / on Ms; taing becene Teath 45 (orshth $100 \mathrm{~ms}$; Reght $100 \mathrm{~ms}$ )

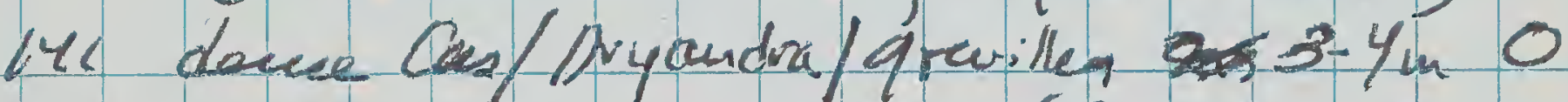

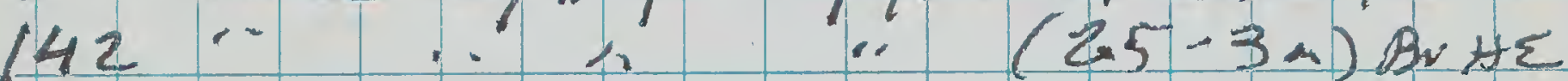
149 . "w pave malkee 0 144 is 1,10 $1445 \ldots$ (3-4ar) $146 . \quad \therefore \quad 4 \quad$ " $\quad$ "ringte

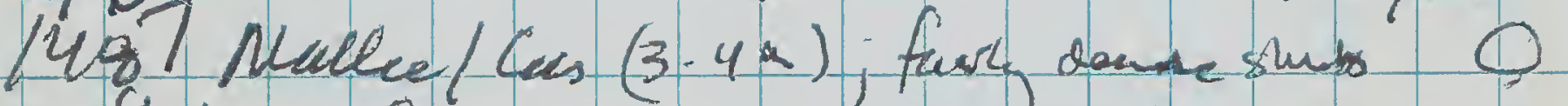

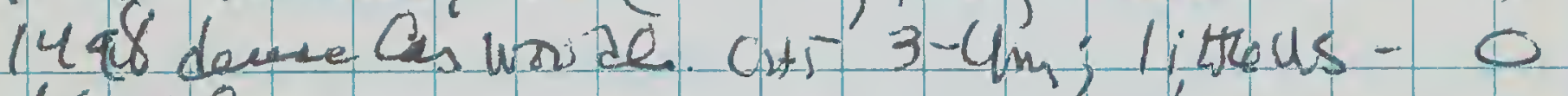

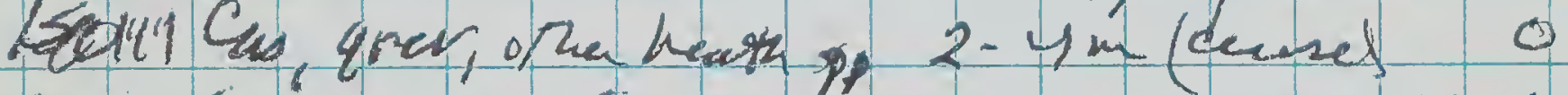

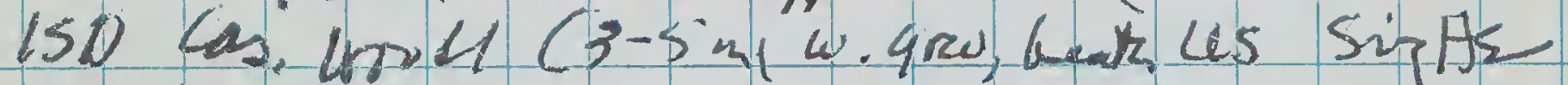
$1521 . \cdots+\ldots$ is Z tal dreer kargar 2-3an

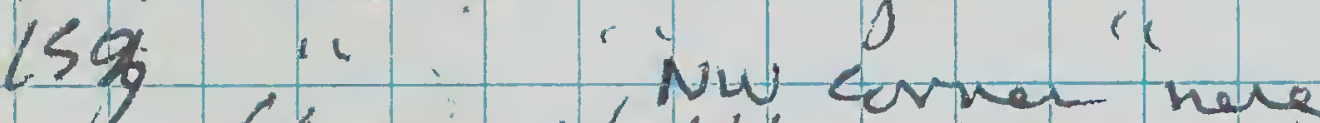
(quapt habbleis jost wi, in retrag) tum S, along w. Wrdar

154 Nallee (2.5-3u) a derathath us 0 155.

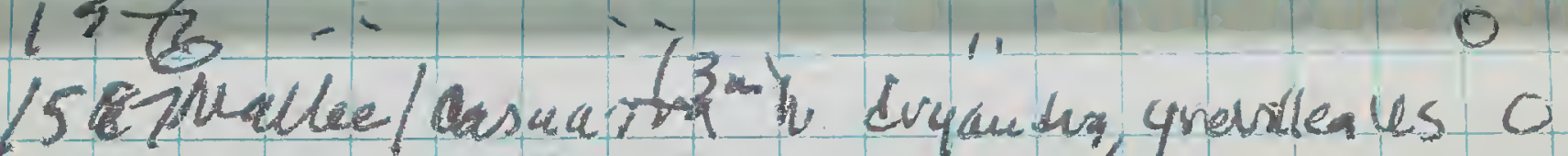

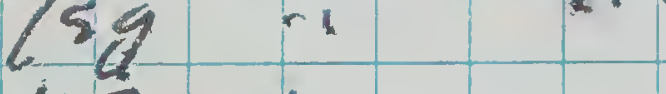

Hô 14

$100 \ldots \ldots+\ldots$

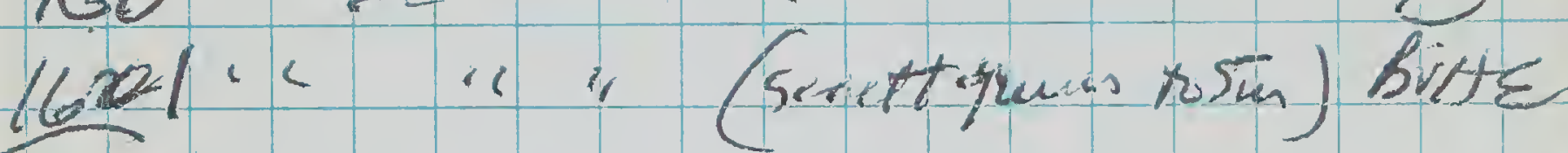

\section{TNI: at vas? raoph intion}

163 open Waudo horde. 10-12urideur us if Cs, swat, ate Bund HE

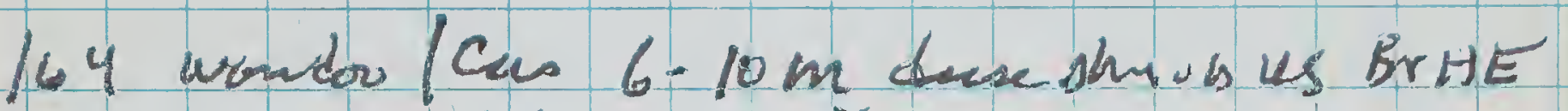
$145 \cdots+\cdots$ [66 Wundot 8-12in demeMs Las; dure Ls shubs ... Weabil . W

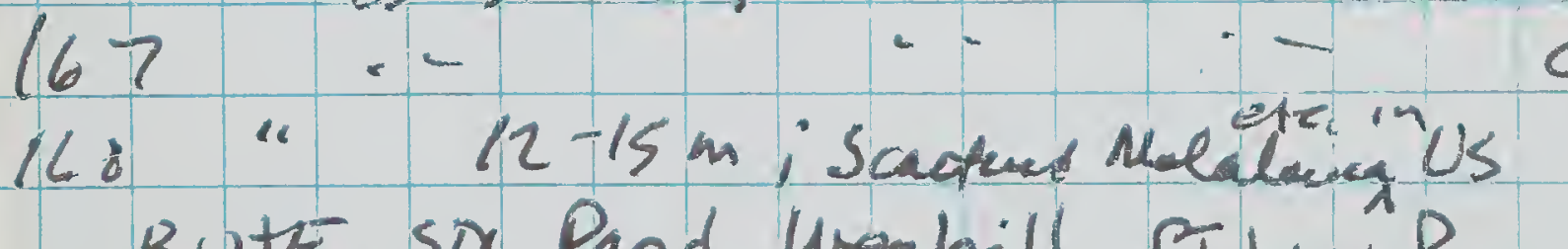
Butte, sir. Pard, Wabill, PTLineP (Rey. Whot the $100 \mathrm{~m} 5$, Q 5 h then 75m N)

16911.1111 0

t2o opean Wandar voude. 15m; arde cownohibs in 45

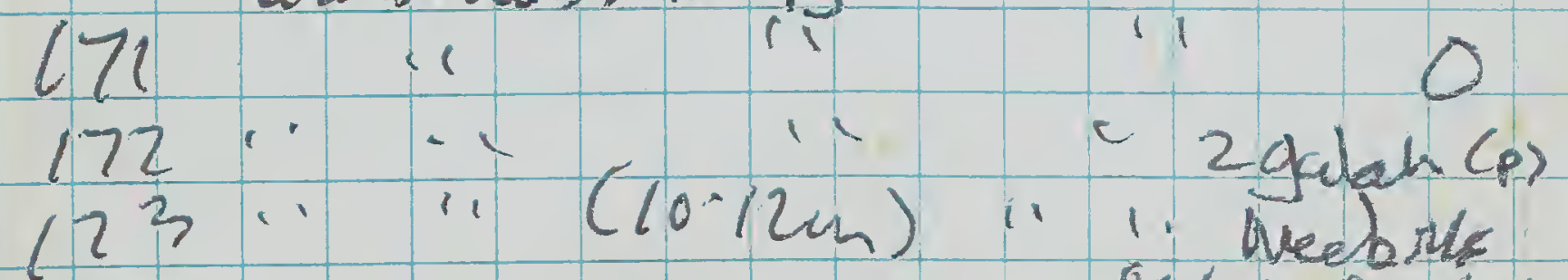

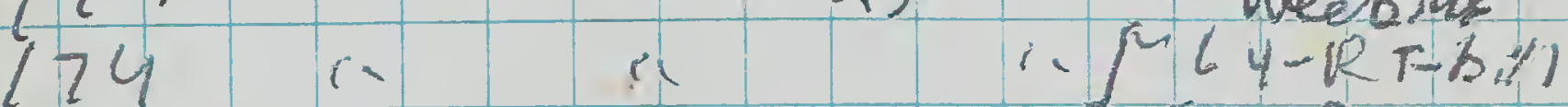
(5Tr. Pandalore

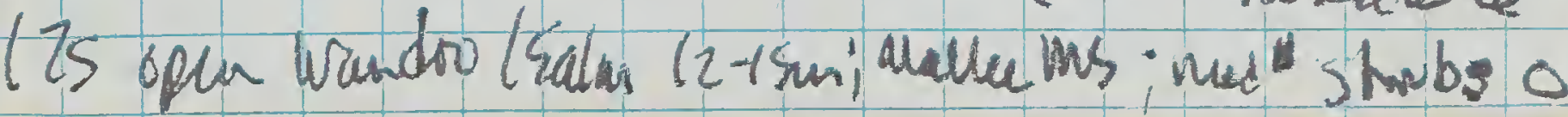


176. open Uandoe 12-isin, saping/Madea Ms, scasts shivilos un US

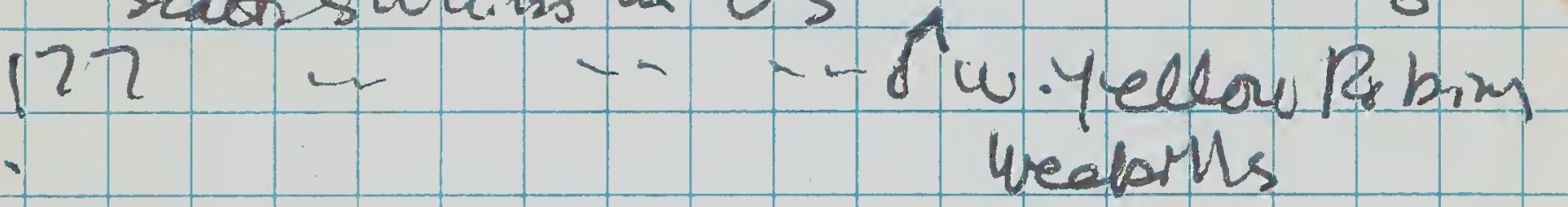

18 opu a N W - C C 183 . Waudor use $10.12 \mathrm{~m}$ w, dune soplj MS; genus

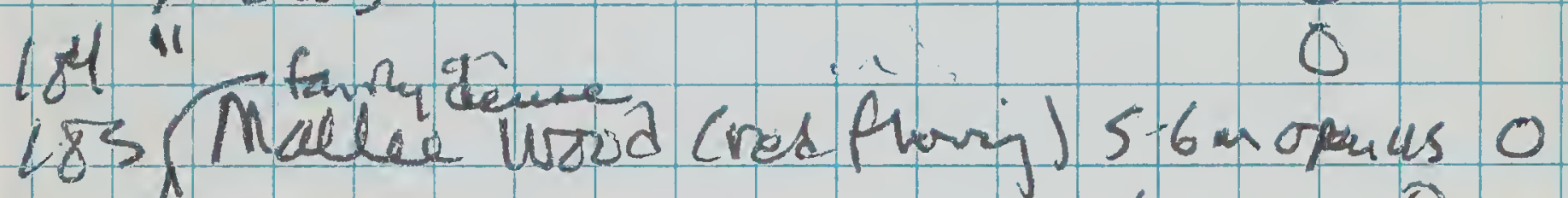
186

187 i 188 :

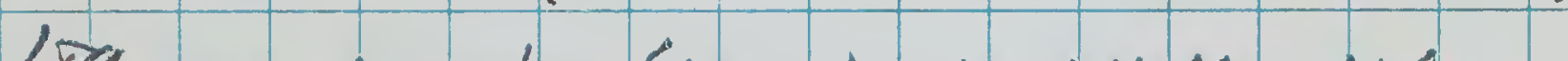

189 open Wrador (kor(2u); amud Mallar MS,

seatent shmps in lus weekms

$190 "$ " 2 primep 2 Galah (p)

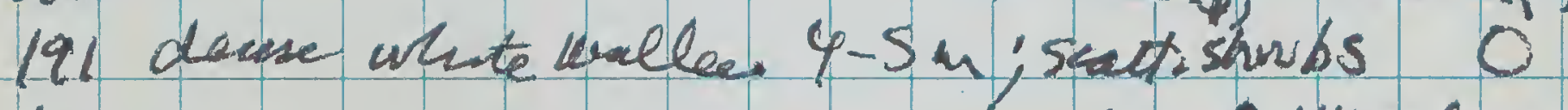

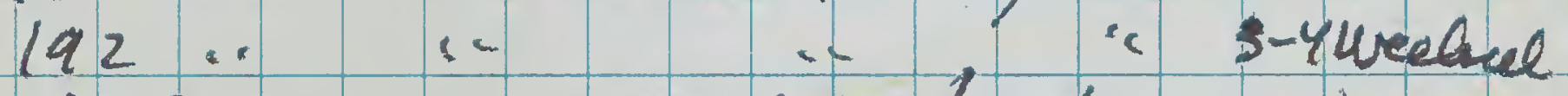
$\log 3 \ldots$ nod $\cdots$ shos o

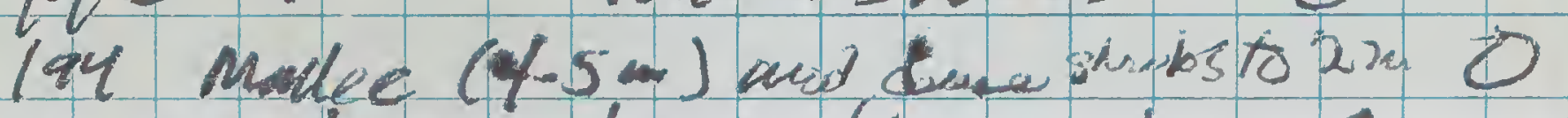

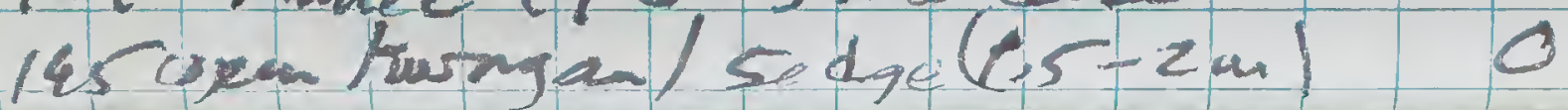

196 "

$147 \%$

0

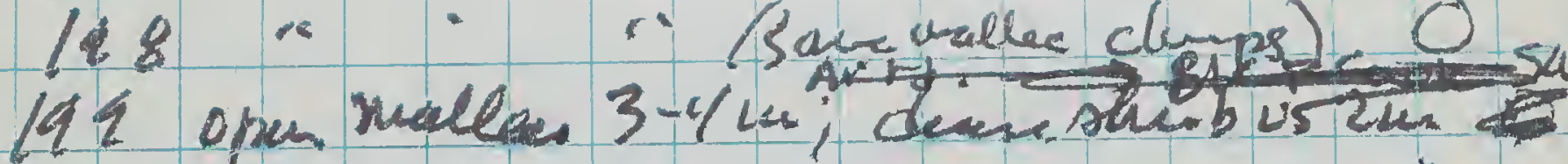
200 a $-a$. a 0 201 demeduch 1.5.2m scat maeler c) 20

CMssino m. Necaver of Kod Kodiu - bar

then havk h: cilve rod to cias EDze cor shith lso us s.

EDEE

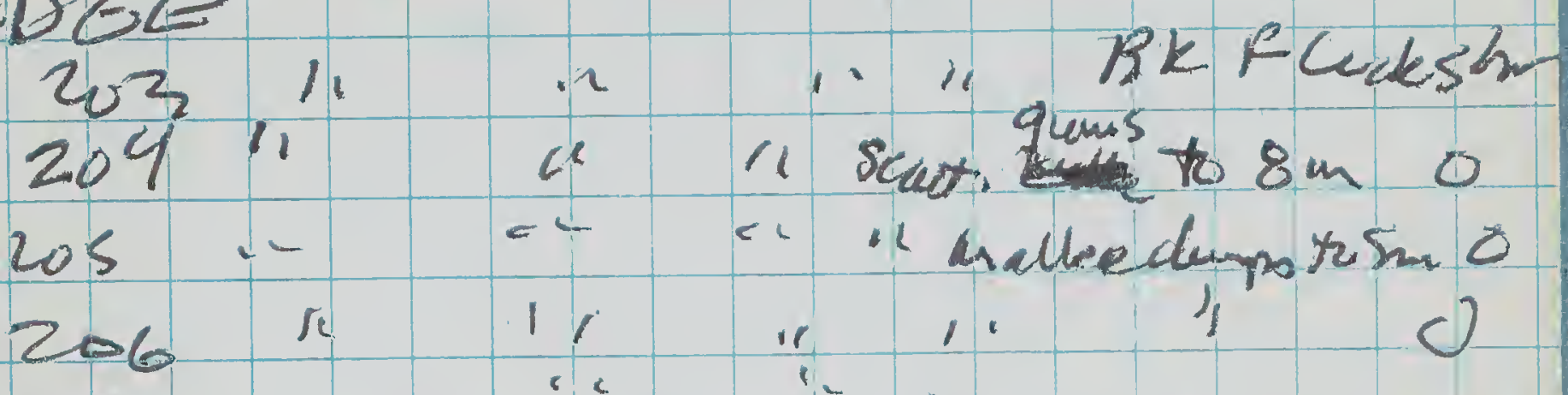

207

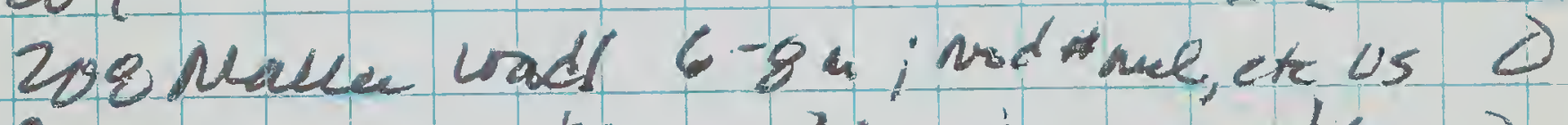
$2 \pi \cdots$

$260 \cdots+\cdots$

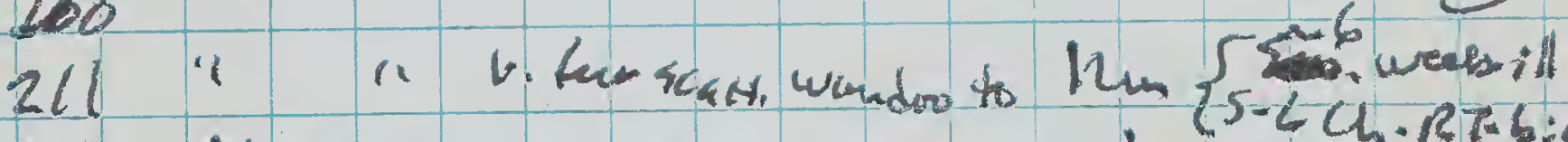

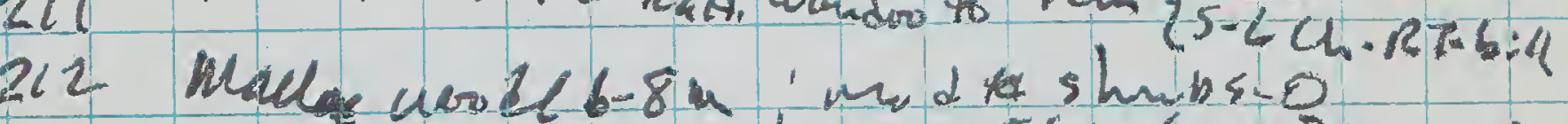

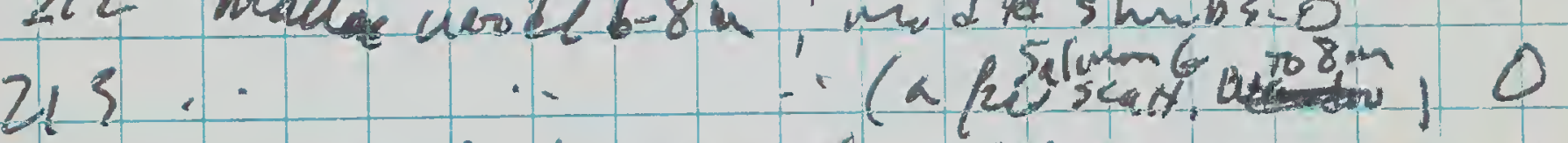
2 in Malle brode 6.8 in ; demo Mef ete us Im 0 215 open Wandro 12 - $-15 n$;MS Matter mod shubo 0

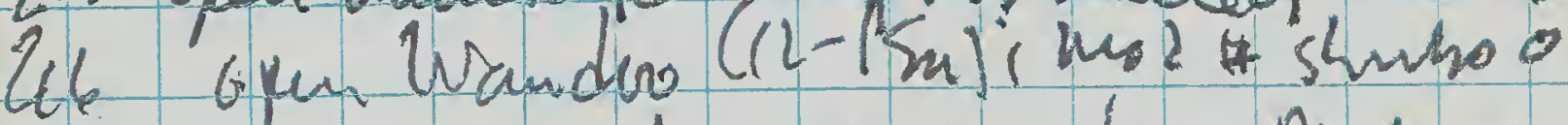
27 made borbl 6.8m; us douremelete 26

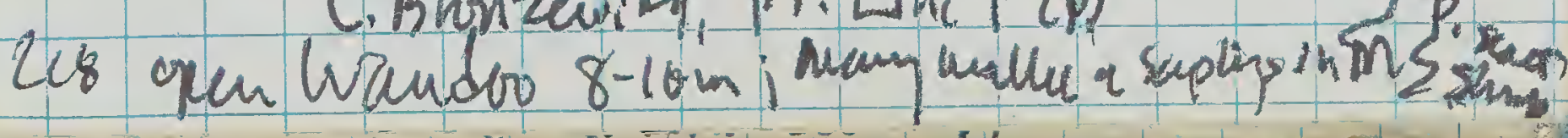




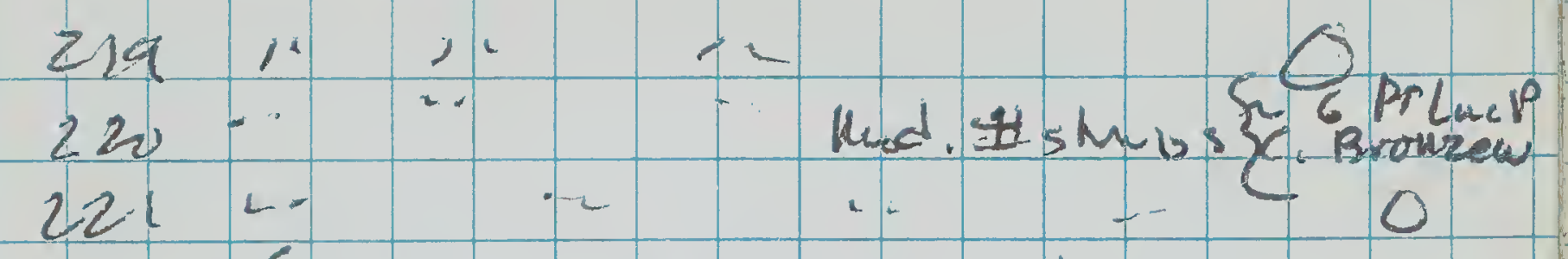

(orsh in $100 \mathrm{~m} 5$ )

222 upen wando worde 12-isn

vud shubs in us - 0

223

12:05 huek at $\mathrm{Ca}$

13:20 lov CSTRO far S. Goiss

Kavial $25.50 \sim 15 \mathrm{~km}$

t5 keleabuarán

2242.4 Gas meurdan $17.4 l^{8 / 1}$.ou

$2330-35$ bigavan of

nataral Ve, W or

Carrabih

¿66kaw s hoss)

- 348 Rr bridgr

qee d anda

350.1 dirt red $N$

350.9 end qut. heath

on N. Side Csritlon

Sirs side of hoy)

Bodalien

53.4 Bodilin

54.1 RR crossiricy

$62.0-63.0$

qad Samue und

S. of

(Tor Wurros)

402 \&5. Gross taninn 412.2 2 mem E. into la rature reserve - reafin,

heallee 1400 de and aveas 412.6 park 


\section{Bivds:}

1. opar J cun wad -10-12m

mod scastues brost chubsto 34

4 Maquie, Weelail/ (Gsur Thurrous)

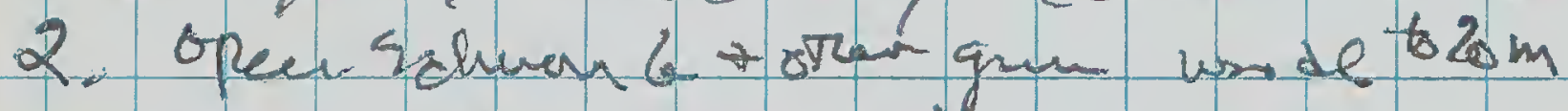

MS of vallee; und s 5 horbs 8

3 .. itany leary shubs $\sim 4$ Curnaurs, 2 pr. hine $P$ (Becthare iscones.)

4 open Salumantotherqun to bon hod. 4 suwbs to 2.3 a Sp-cheatad 15 2 inc. tholl

$5 \because \sim(5 \mathrm{~m}) \quad 2$ sm Padalote 6, $14 \quad$ i. 4 ch-r T-bill, weebill

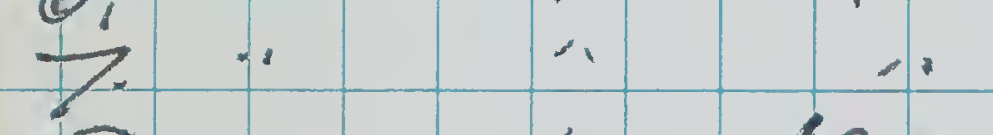
$8 \cdots \quad 12 \mathrm{~m}$ jomebrusto $25 \mathrm{mo}$ $9 \quad 1.9(\tan s$.

9 (15m); mudts surutos - 0 ( 2 qalah feav over)

00 in k1 i 0

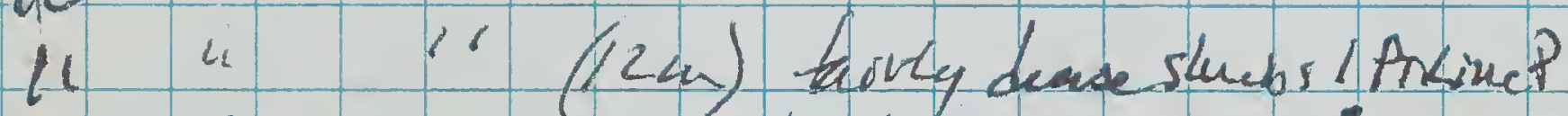
12 il 11 jinod st shmbs 6 curtbill

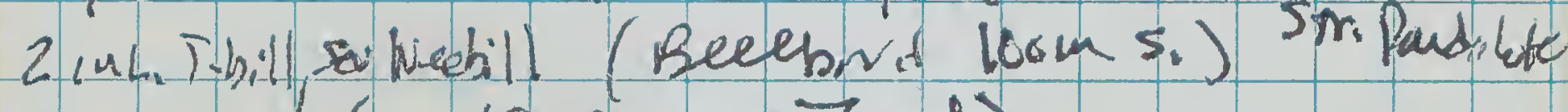
( 2 vavas vertecad)

$13 \mathrm{~V}$. oper siluito $20 \mathrm{~m}$ w. mod brash clemps gakh

Grsh Thil Belluwid 111

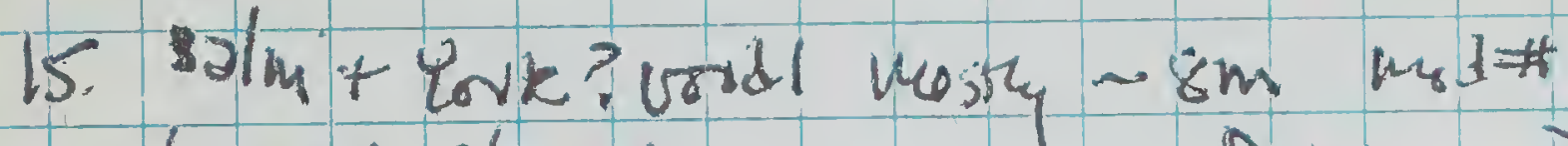
monar elempe - PTilineP 16 "York"wh 5.6m ka shwls - O (them N. here -at edge of 5 kmbtove)

12 Solum G. "Yake wrodl. ISm mad shumbs - O 18 in ic $210 \mathrm{~m}$ " "C.Bringening 20 "York" wallee son j faily deren harks -0

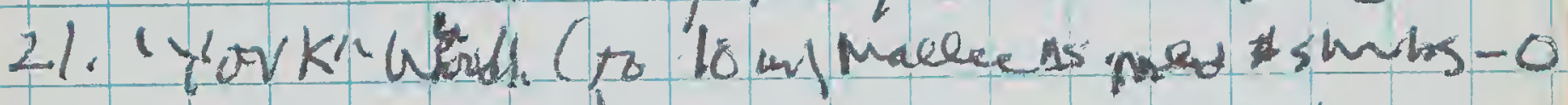
(bouer to vicurity of cars)

\section{Continue $N$}

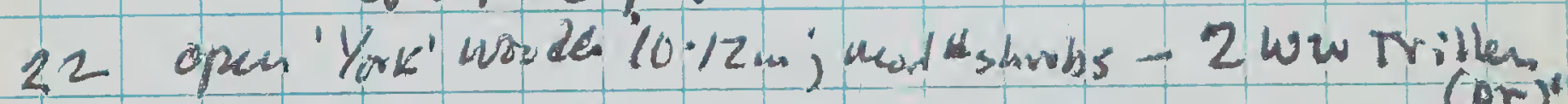

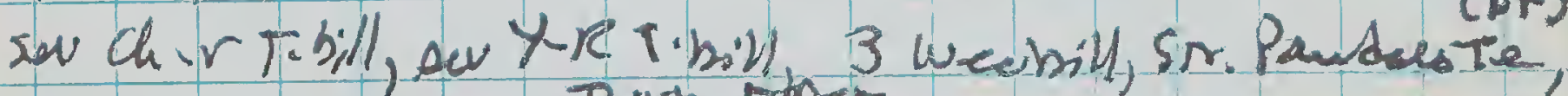

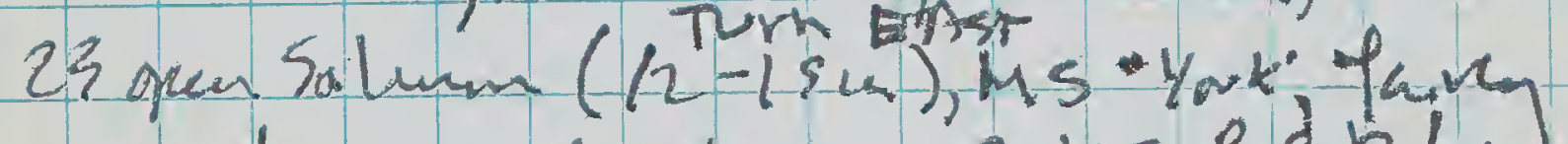
deun wumbs 2 uue R.CRobin?C.Bronzew 24 opar Sien to zom; Mod shubs us - 0 . 25 . York' Molley b-1 $\mathrm{m}$ ikny duse shrobs 0 24 ropen tare Salum (2ow i m 5 of 'Vove.

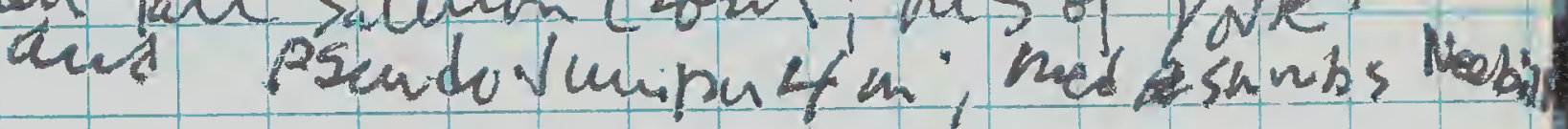
27 a "( ISm); SaneMs, Us - 0 
28 Viopen gavk like Solum g; Ms of Snotund 'Yowh'me Raeudo Juipu-

Sectured 1-1.5m shu hs -

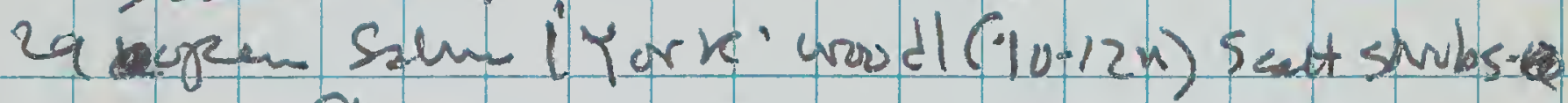
(Boubird zoom N) ZY-TMiner Torn N

30 Vopren Silm/4nik werde 12-15m-MS

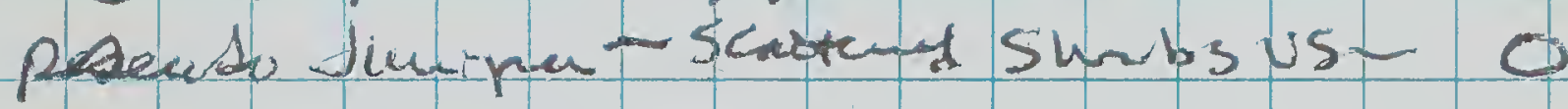

3)

32 Malle $3-41$ juad shano- 0 thenolvest

33 Mrelle) Cos 3-5 m; tavlly duse shim 0 34 ic

351

36 open Moulion. dease prows

37 like 45

38

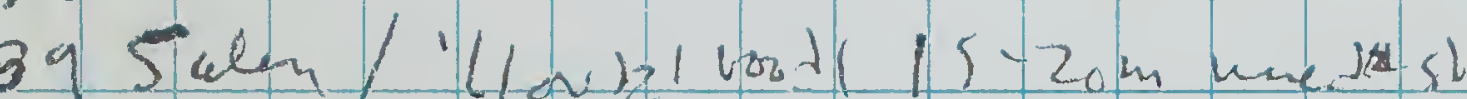

40 : " " MS psado Jum;

mera shichs - 0

41 Melen $6-8 m ; f$ dear $45-$

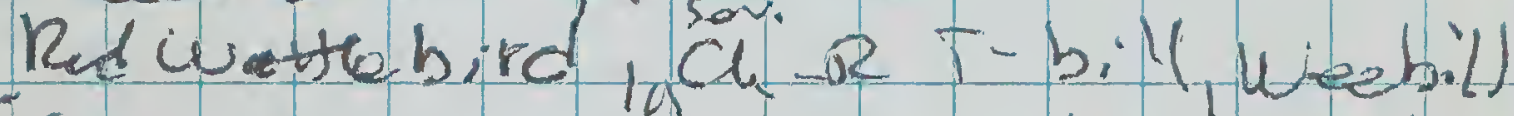

2. (ashini-200N $W_{1}$ ) |NL,T-bil|

42 open mazle u. faviy danze. shims CAT 4 un o

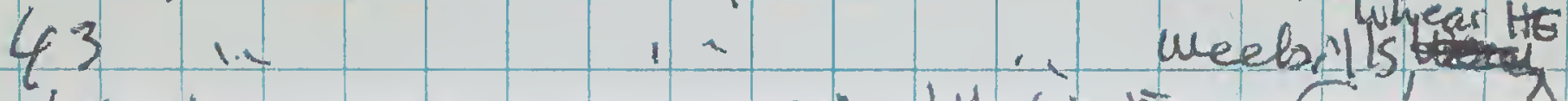
441 c \& - Mod tushrobs

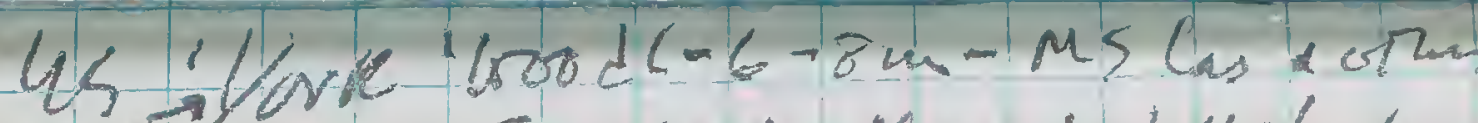

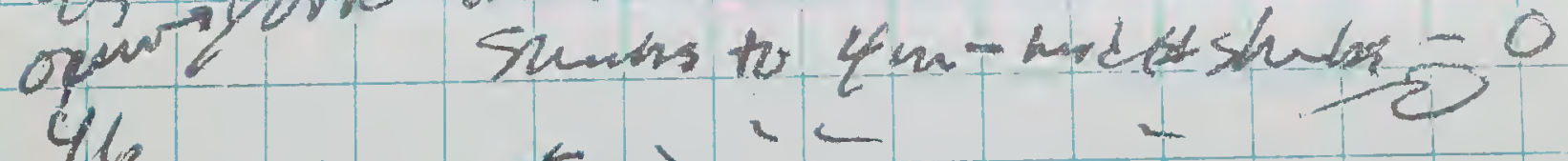

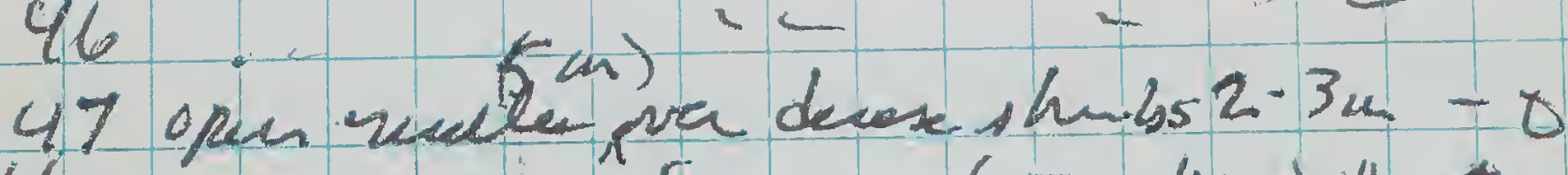
$48 \%$ " $\quad r$ urebills

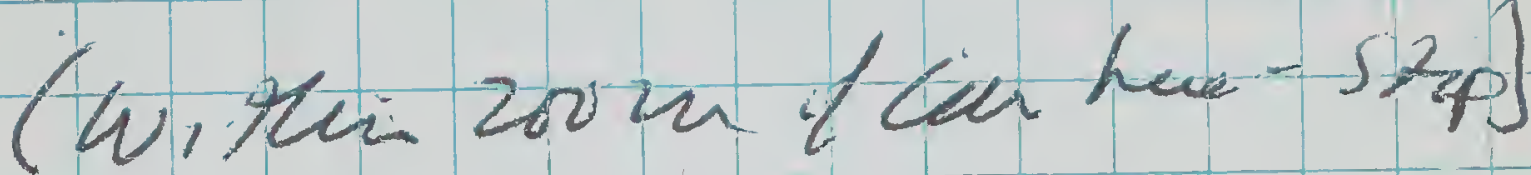

$$
\text { 3 } 17: 20
$$

2413.0 - backet paid rd.

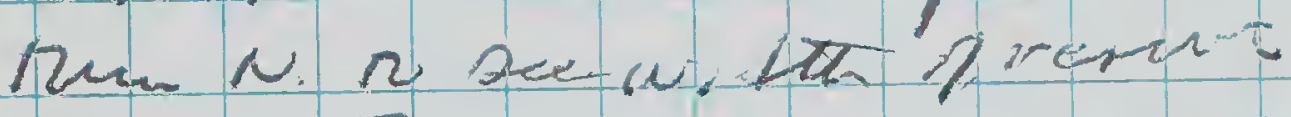

24/4. 7 N. hovder w patbock (parbeck also to W. f rewr)

$419.05 . \mathrm{end}$

$$
25.51 .25-81600
$$

3 Nor.

leare Si cooss at 05.45 192,427,5 for Yellowdive $(10.8 \mathrm{~km} \mathrm{E.)}$

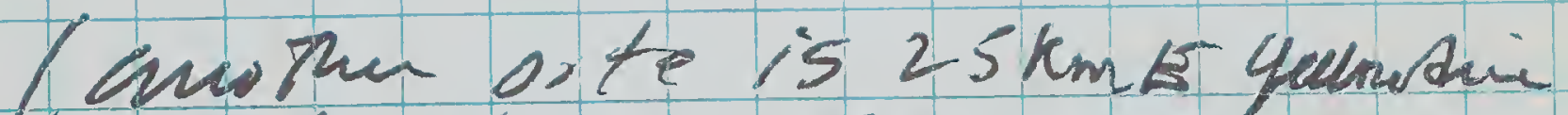

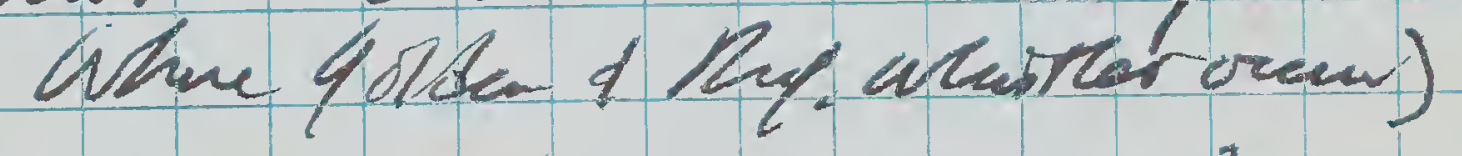

purtithoslo (4 ye) \&3 of bust 


\section{$3 \operatorname{Nov}\left(\operatorname{con} t^{\prime} d\right)$ \\ Drove past Gilkatsutuita on te aut kad to dow ble busk}

473.2 propen stop $06=20$ weather exd, deade, breezy

transer suveqa

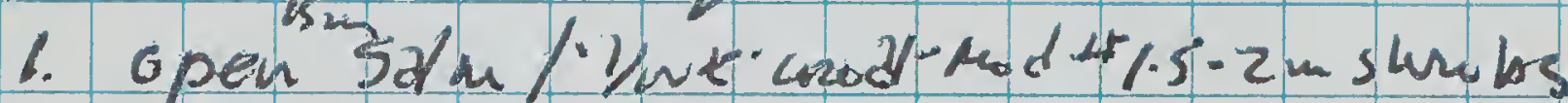
y-pluned te, Red Wattabive arshish Bk-t Woodsw

$\bar{Y}-$

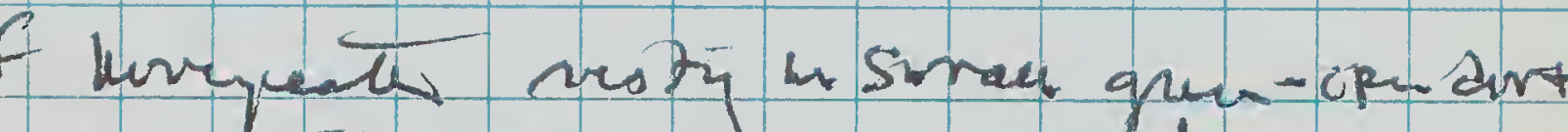

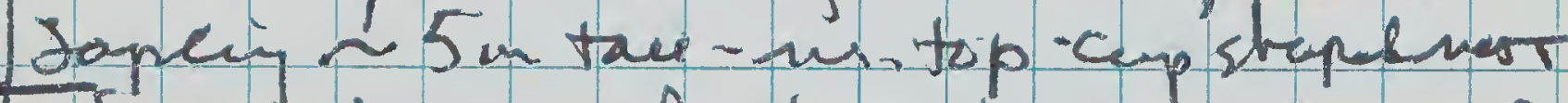

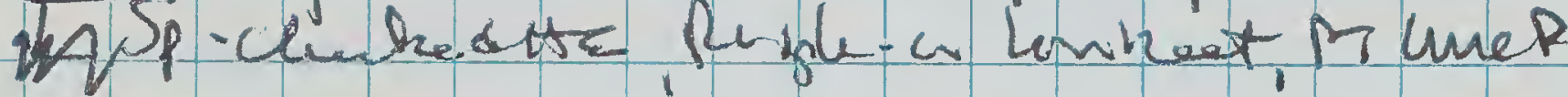

2.2Y-plem ditte Giberrsurster

3. Open Gam /2-15u; madt shins $12 \mathrm{~m}$; bavedor Wille Wratail, $y-p !$ It

4. V. opar gam u. Eaciry déne In bushes

5

- bromzeang Ar WW Triller

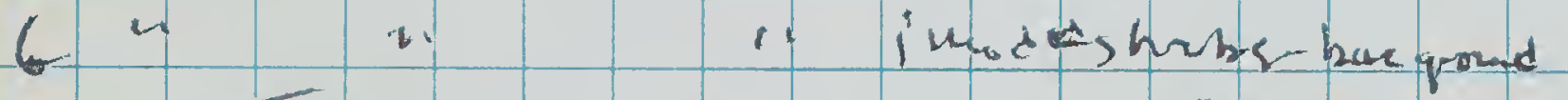

SMite, Y-PI. HE, SP. Conceked HE

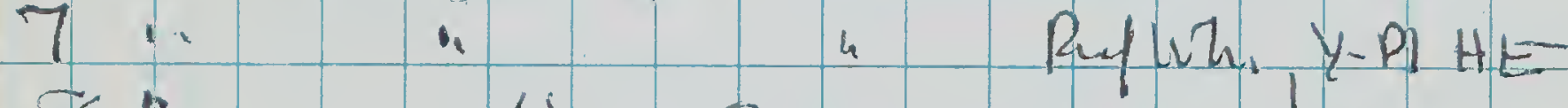
8" " saa shabclumpiuts of bare povend; R.CRobm, sewChurT.bills, Whesbill

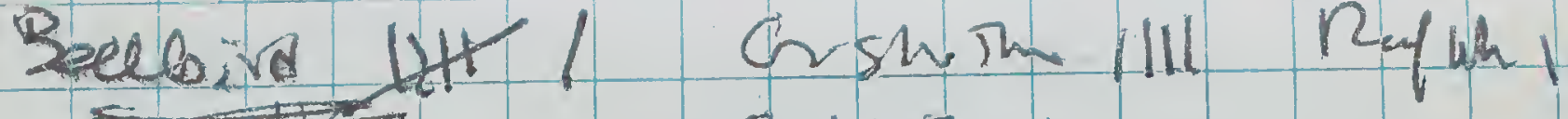
Gilwh

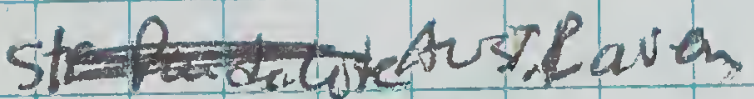

दctera

Turv east

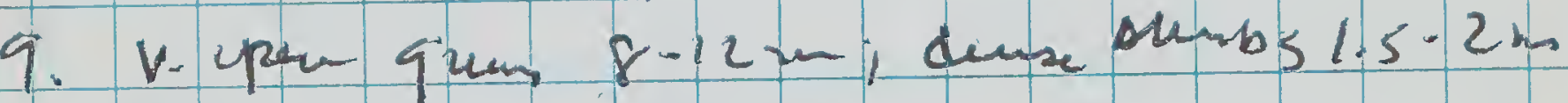
far anit bavedovt - 0

low Paring dure Acacialete Serrb 2.2.5n SujtE H. Mallee 4-5m in Mod $*$ in stanbs us. 0 $12 \ldots$ " " sate shubs -0 13. opan Mallec 6.8 m j Ms Cas., Acase 2.5.3.5m und sumbs in us - Hee Robiog zRedWattrob It oper gun words C-8.miF. denze shurbsto $2 m-0$

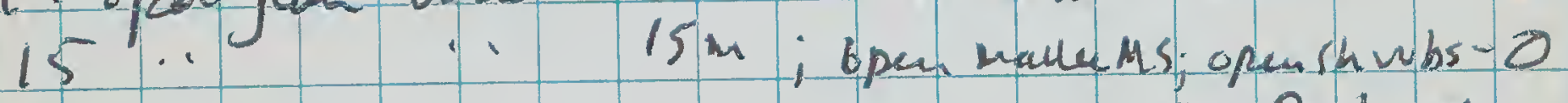
Q.. 12 a son Pardalote

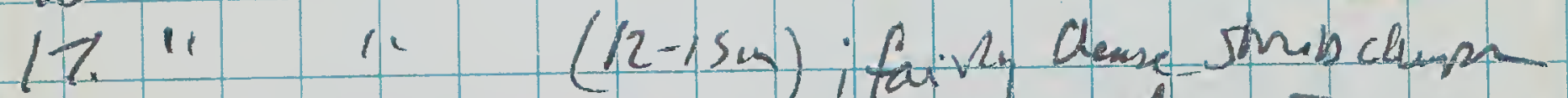
ser. werbill, $2 \mathrm{~mL}$. T-bill, ch-r Fbill

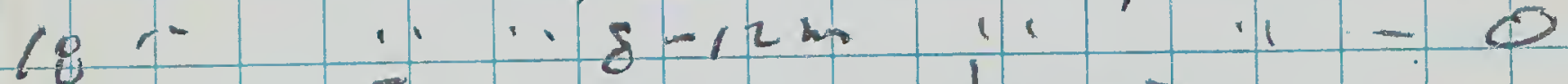
[Tuv M. Tound hary]

19. opd qun wrod $8-12 \mathrm{~m}$; duse stumbs to $2.5 \mathrm{~m}$ 2 heelsill

20 ic $\infty$ 2h "York" undl. $10-12 \mathrm{~m}$; wade shing 


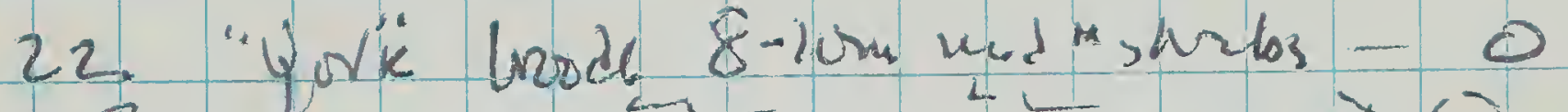
$23 \ldots$ me

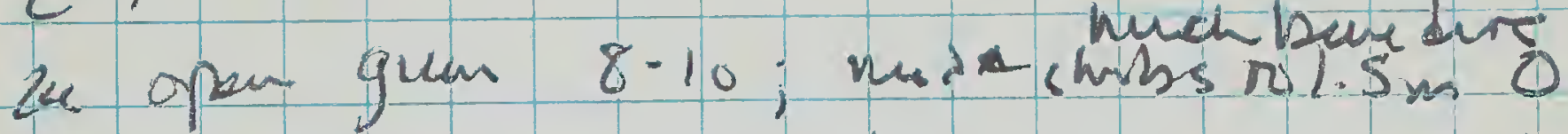

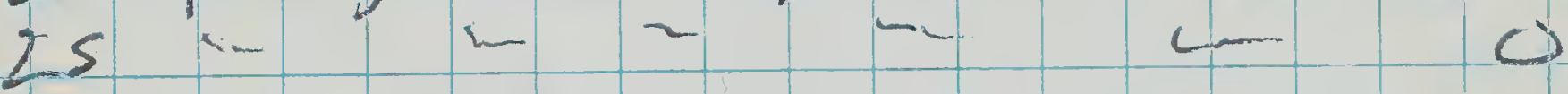
26 - $\rightarrow$ h th a weebial 27 V pargum .. .. 5cat stumbs - 0

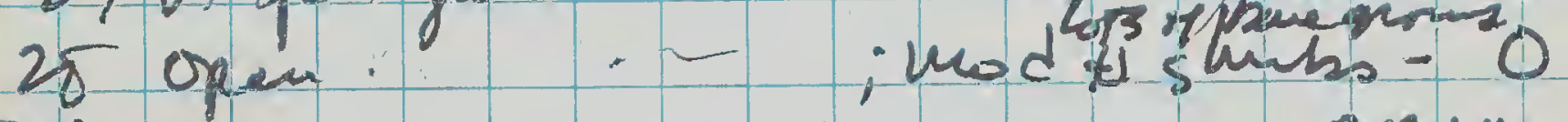

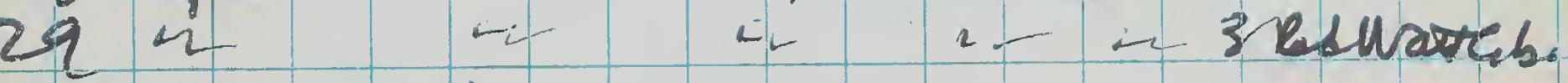
30 .

31 opan quen 8-lom; MS a pardo turpamod shubs; much bive gand $y-p \mid$ tete

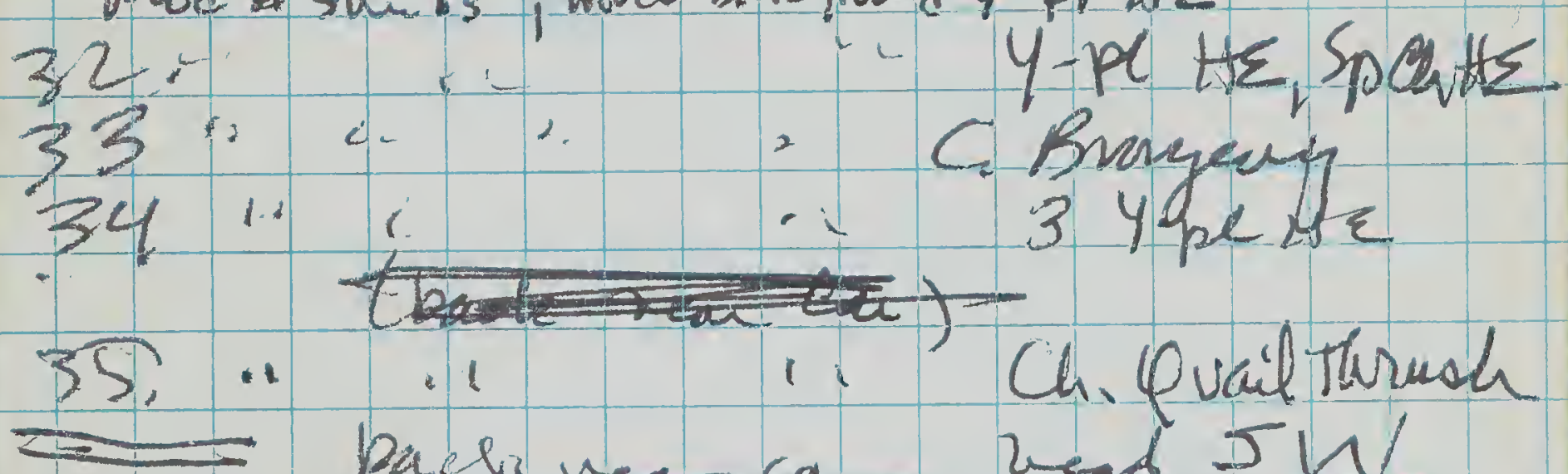
baclinar can wead IV

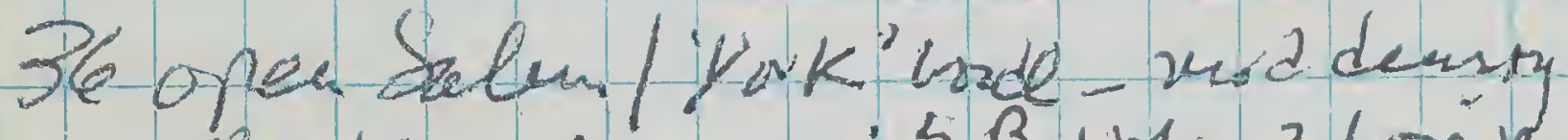
samb 1.5:2m:5 Basblen, 2 Lorikeats $31 \cdots \cdots$ spcattrfat 32 -

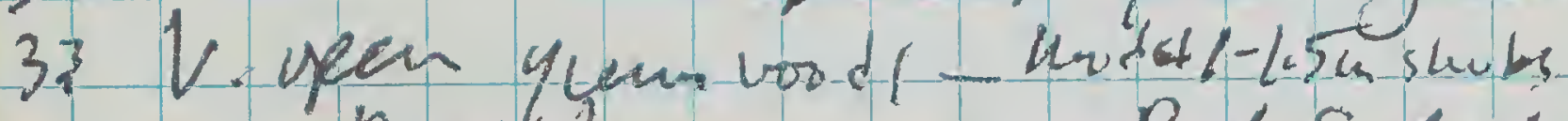
Bush hau qumes - Raf. Singlaik 34. openquen 12-15uj mod shubsNuch busey ona 2 PT.Lic P. (p) pr Ruloes tree cuaren.

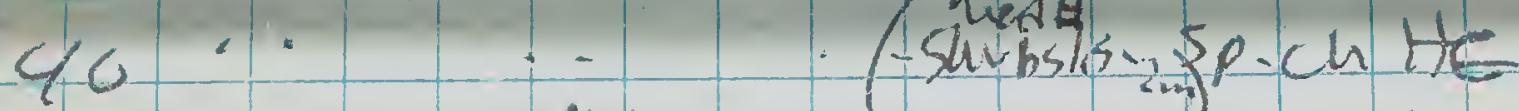

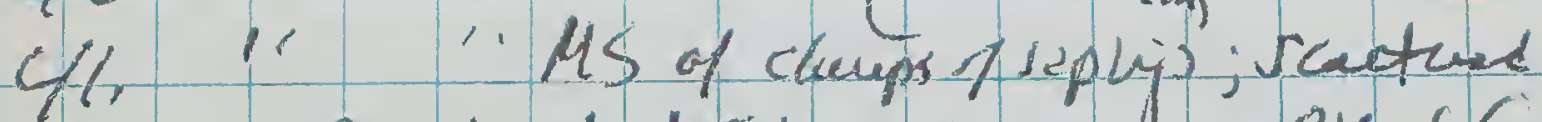

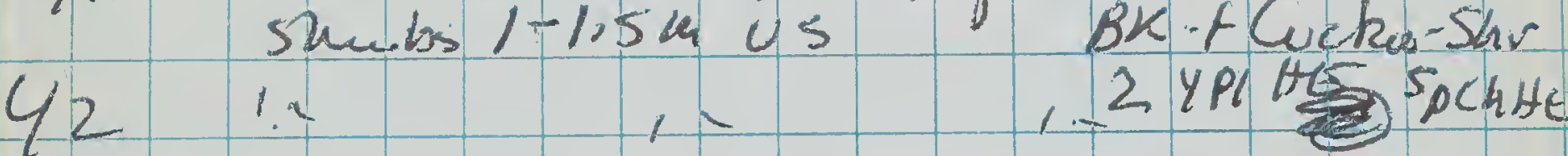

Note: Litte Eqgle hestig $\sim 50$ a 5. of mansect the: In kn stich vest wir, sam glean Eualypt twip $m$ top - in taviz of Solvingem $10 \mathrm{~m}$ up in a 12 -i4m tree. Two adeets souriy arikead 43 .. . ' v vaw wintion 44 Mallee. (6-7m) shorbs - una houeqound $Y$-p) HE, RaITreecreaper des 45.1 .1 "RCRobin

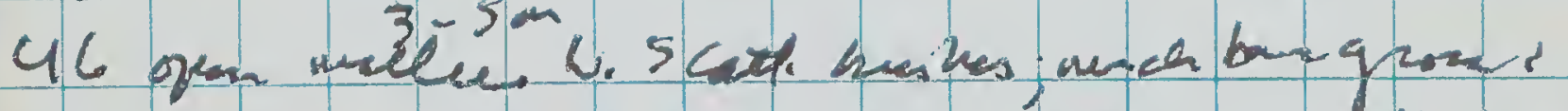
〜 10 ch-r Thill, th Y Y S-bill Y.PLHE $\$$ Redithroat

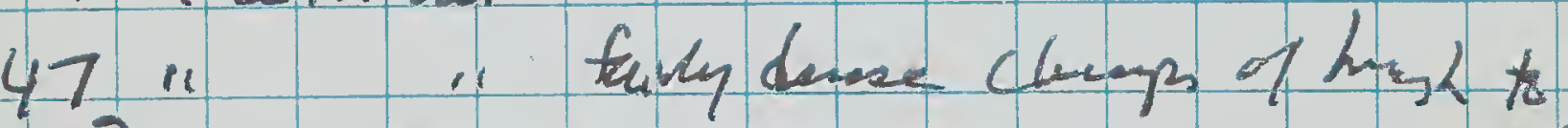

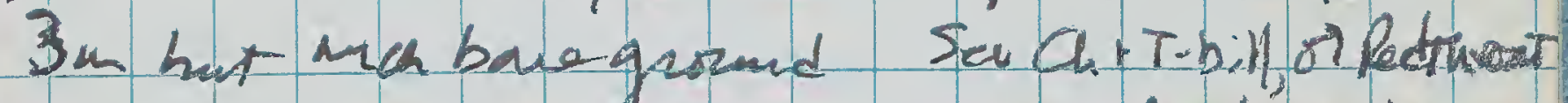
48. " $"$ " 3 sp.chaked tE 49 " C. " ser Weebill Jo 11 u " 0

51 Vopen quu Wrode. V-rim; Saqferd mallee aud hoshes Grecrobon

in apu Salung, vadle. $10-12 \mathrm{~m}$; sut busk 0 ar on tw 200 on 5 
53. Viopen Salm Uerd/ /2-15u; had \$

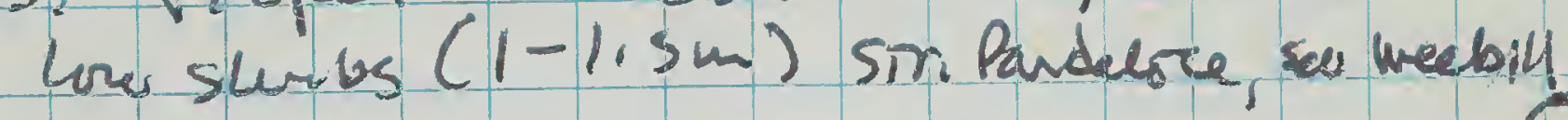
$\begin{array}{llll}54 & 4 & 4 & 4\end{array}$ 56 - 200

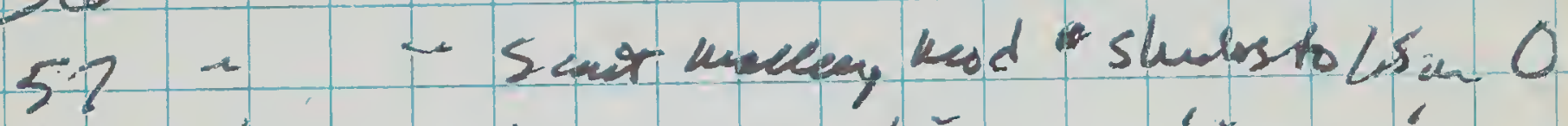

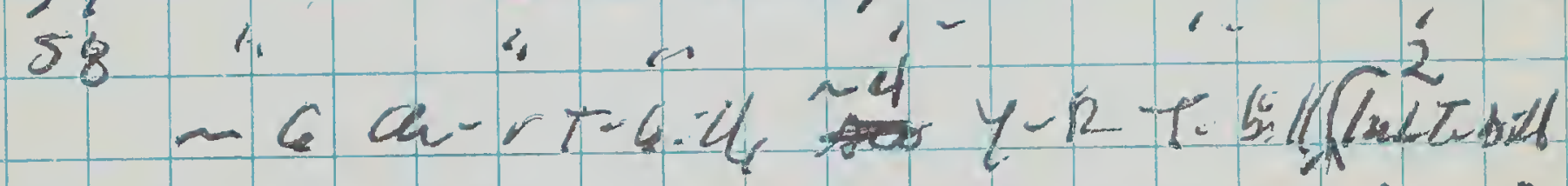

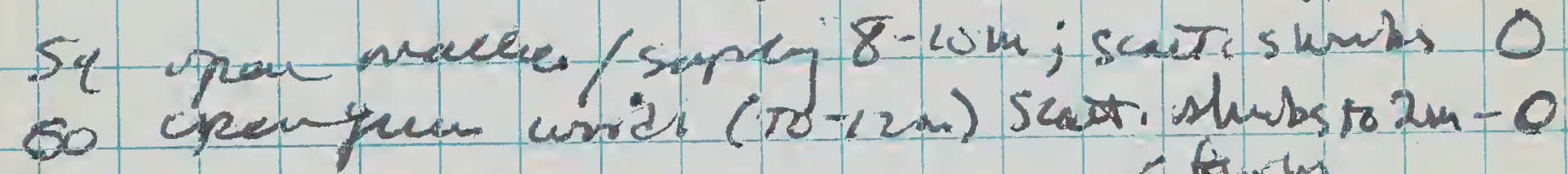

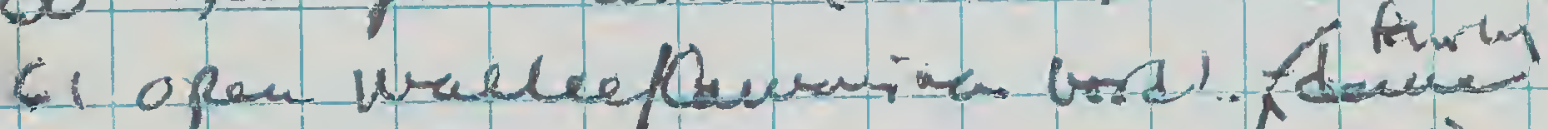
US of Alums $x$ Cas to $2 \mathrm{~m}-\mathrm{C}$

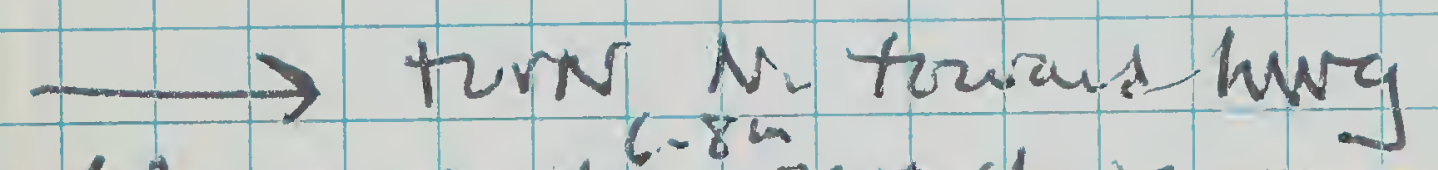

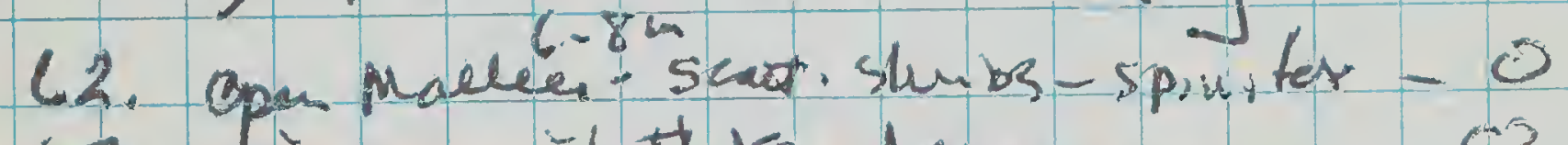

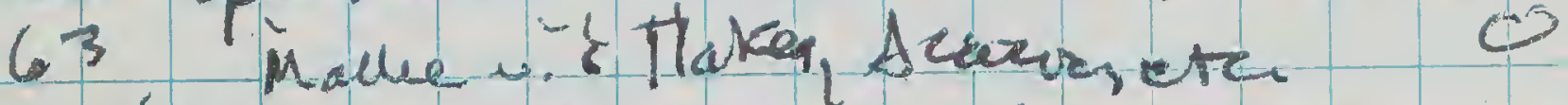

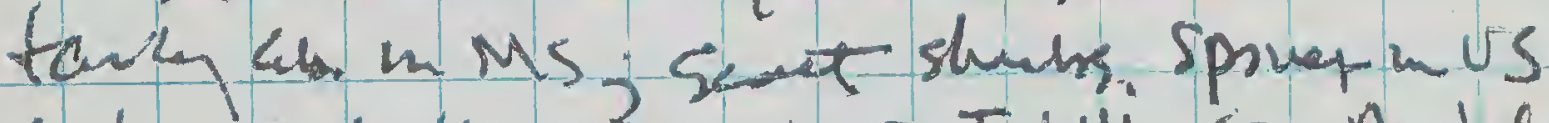

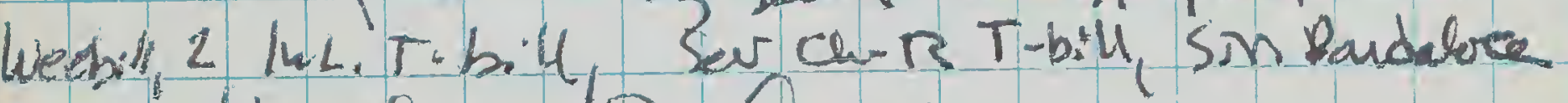
Hars Beuds Bn Curkoo

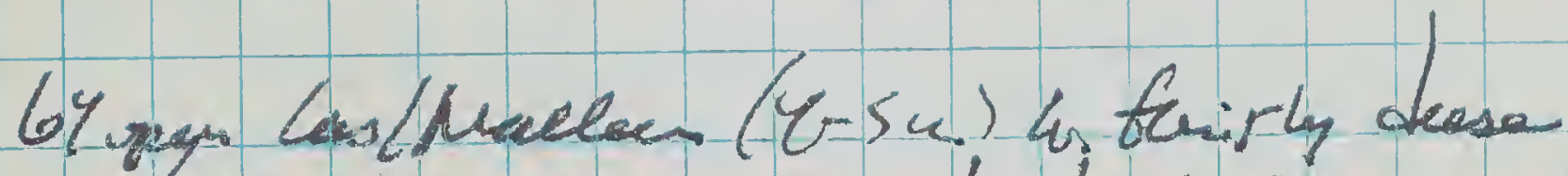
metpoting cassosen shuibs 2-2.5m- 0

Gs Deuse Cas/Acawoletr, scubl.5-2.5.40 0 $66 \cdots$.. a. xat wellee domps 0 62

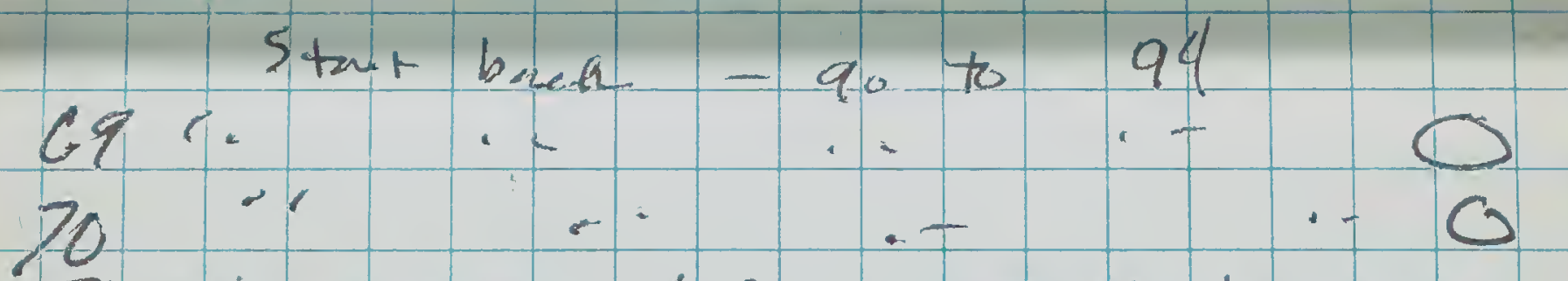
II open wallee 6-8m jund sthubs in us - 0 22

$\nabla$ (beitsind 100 an

\section{SE}

74

75 $-$ $-\infty$

76

779.01 Mallee $6.8 \mathrm{~m}$; Scruar shrubs

To orem salu cond 8-12 miknd shales 8RCRibin 29 solen wordl.72-15m; mod*1-195 a shihso

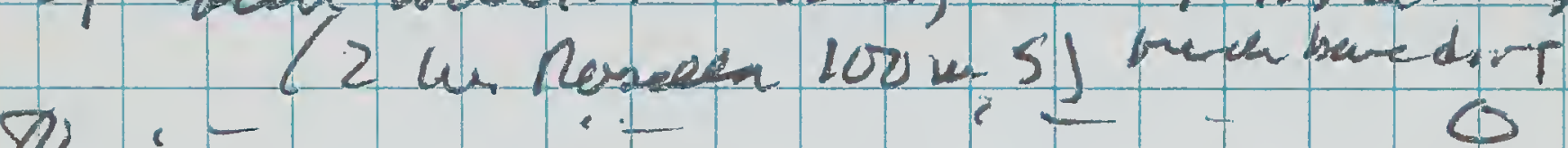

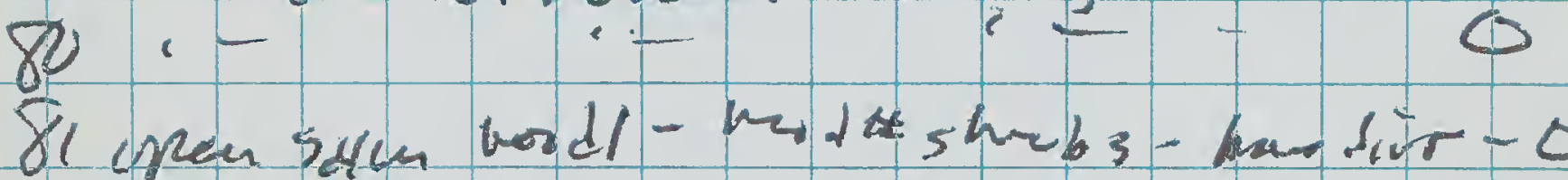

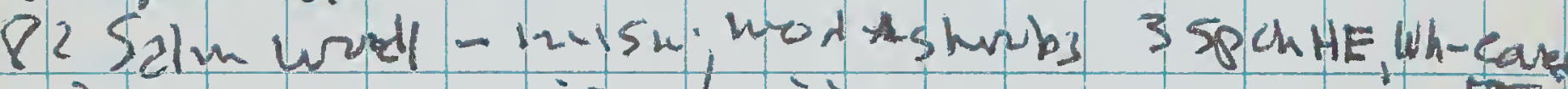
$83 \cdots . . .2$ PT. Line 84 Gpen tase 'York' CAT 15.204j read \# 1-lism sunbs $6 \mathrm{~W}$. Roselasp Ch HE

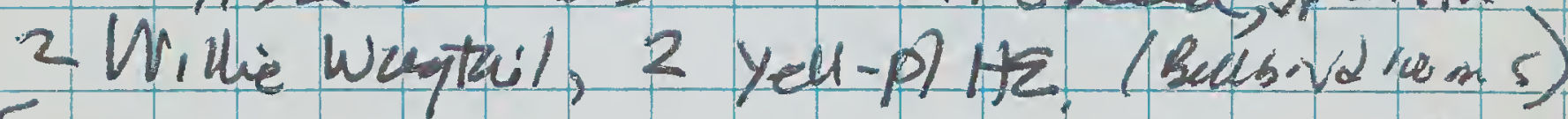
$85 \cdots \cdots$ 86. " 2 Yeaplite (Can is formang) to N. Side of 9:50:4an- 


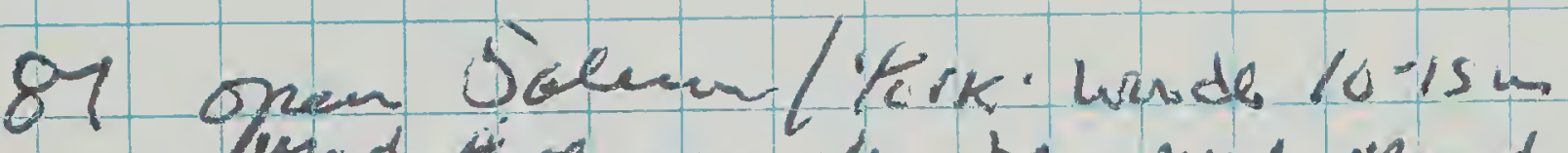

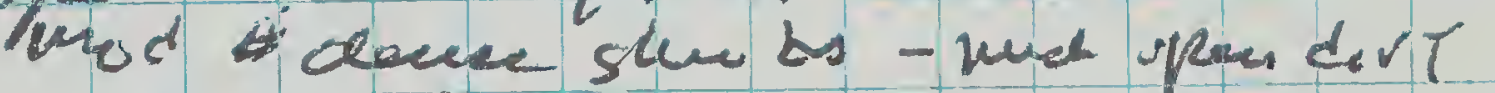
2 T. Lin P.

88 a

89 2 randertirds

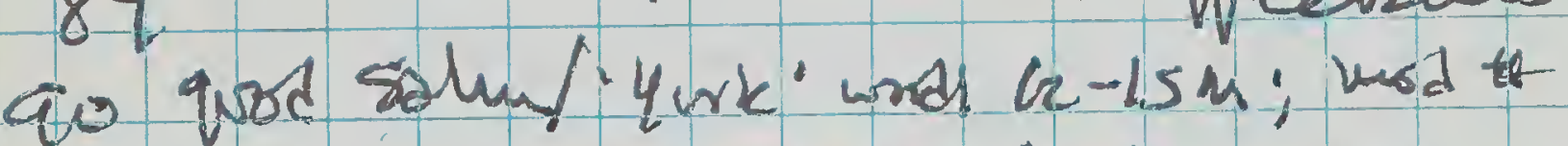

saplijs in Msisont suras us - 0

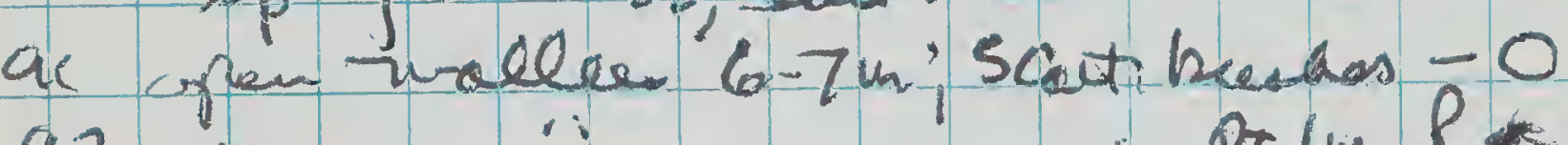

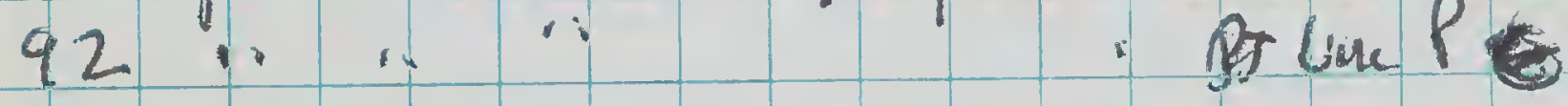

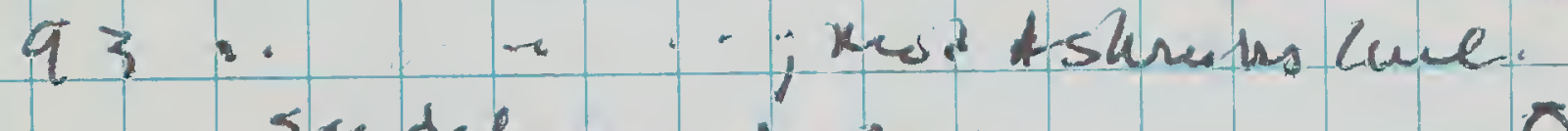
sudalwid to zurl 0

ar

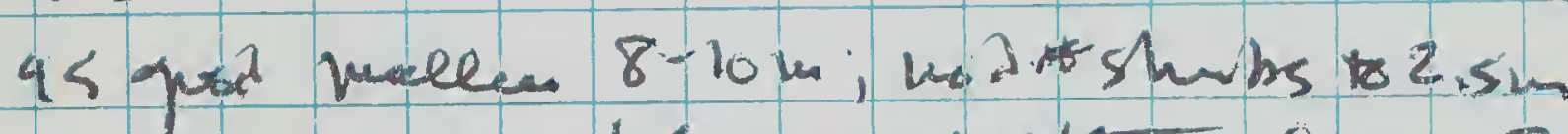

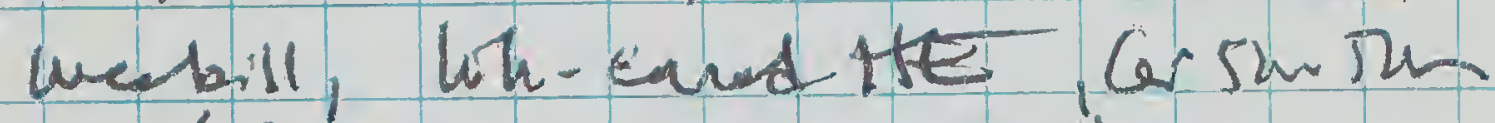
(Bounial lsom W)

$46 . \cdots(4-6 m) \cdots$

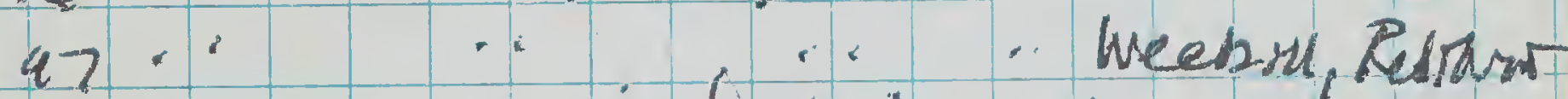

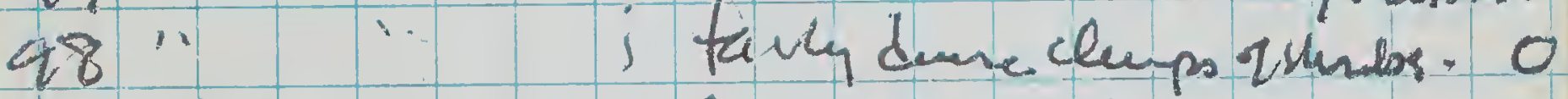

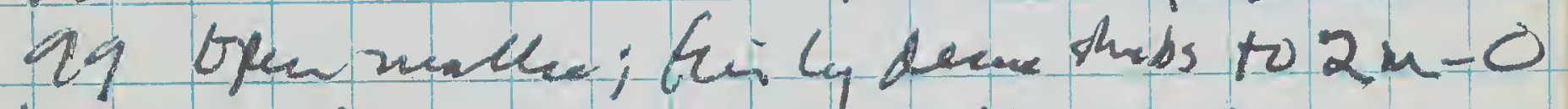

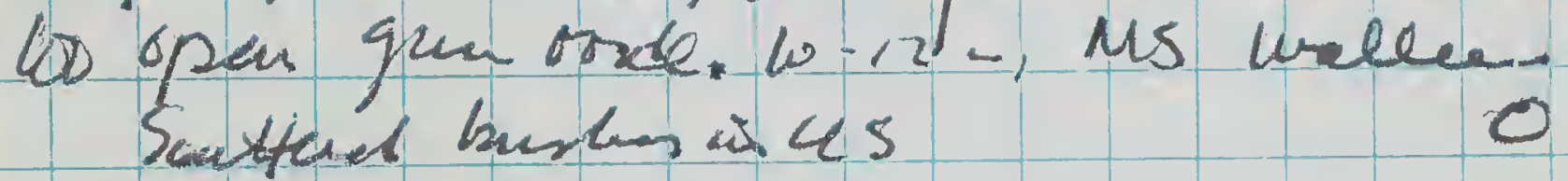

Bach ro Cer at $10 \times 15$

mileage $247 \% 1$

leas lov to finc ander sites

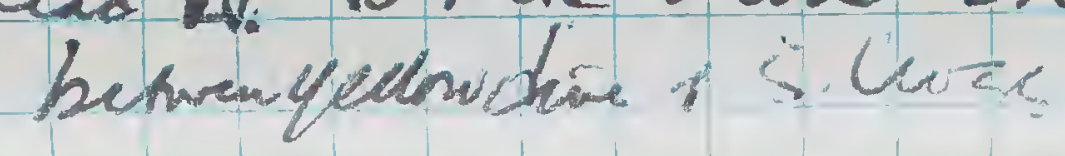

\section{4,5 Sta.}

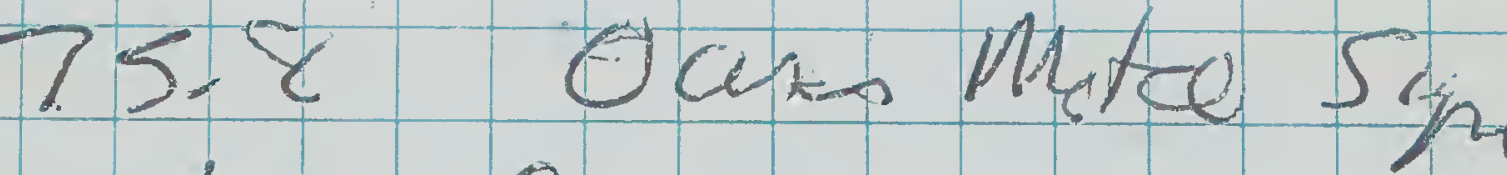

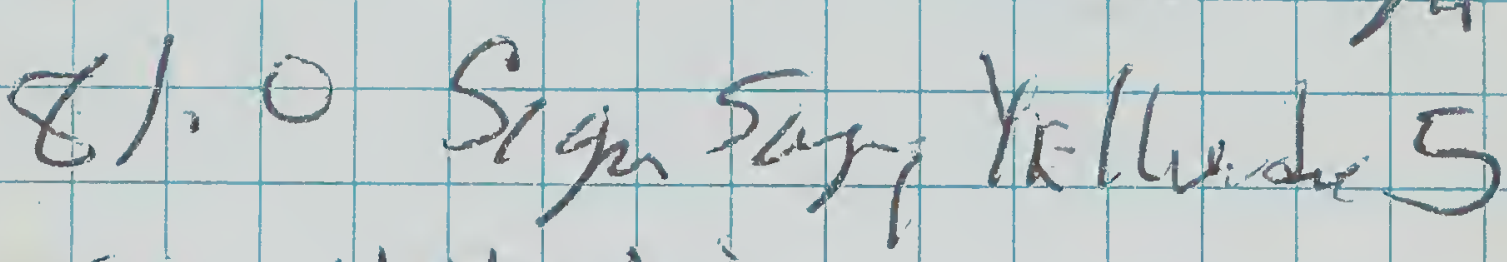

6le Ylowbire

q. 9 twe buak $E$

97.0 tarn Siandertat yeunderes

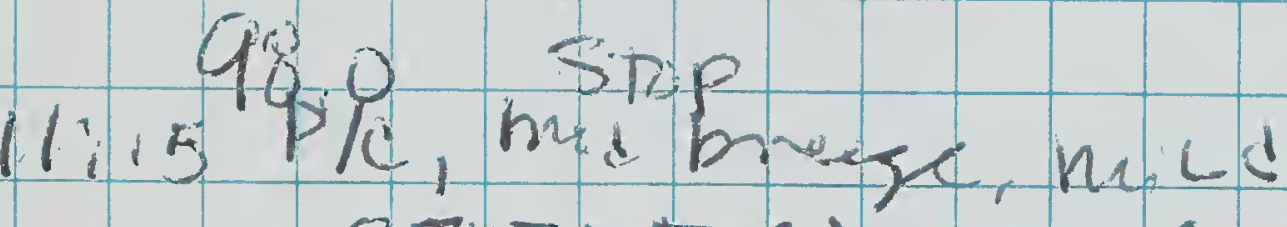
5 rertout gain elast from track

lol opar salm undi $\sim$ Lo ms modesho. Is mash bar pound. 2 c.-R T.bill

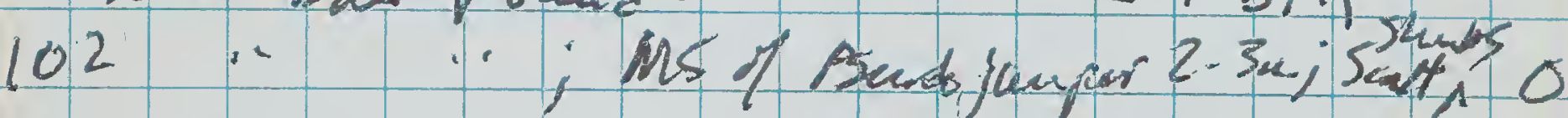
$103 \& 11 \cdots \cdots+10$

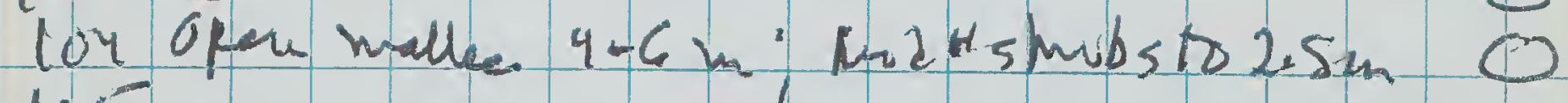
$105-$

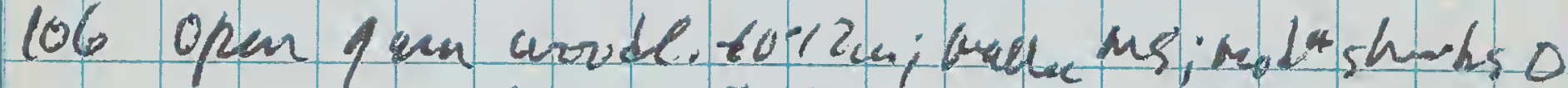

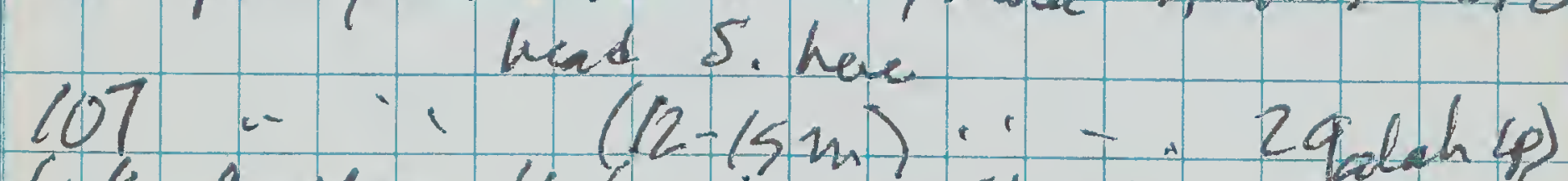

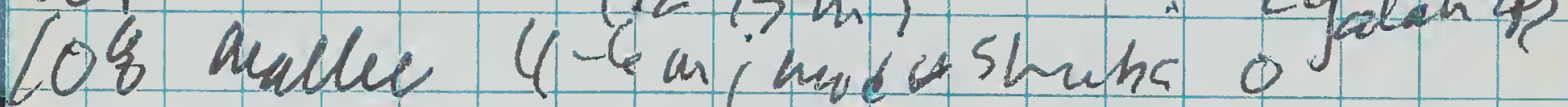


[110 sturts. foumen]

UV. open salmon undel. (Eme no welle)

10-12m; mod shims 0

111. open vidlee-teny densesuss to $2 \sin 0$

112 dewow strubs 3-4m (Cas.-like) 8

113

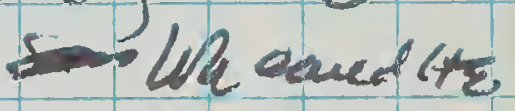

$1 / 4$

Ls

$\because(4) \quad(2-3 \cdots)$

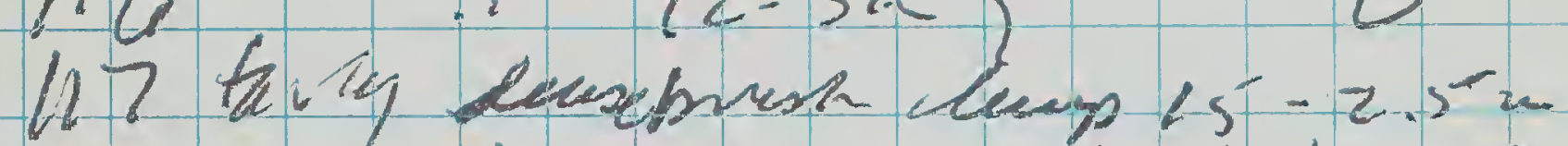
a spinifex haturen wedsil to (Mniluih kevrdus trugled an stry une deand on tail)

118 h 0 0 «.

Turn west from trade prusua

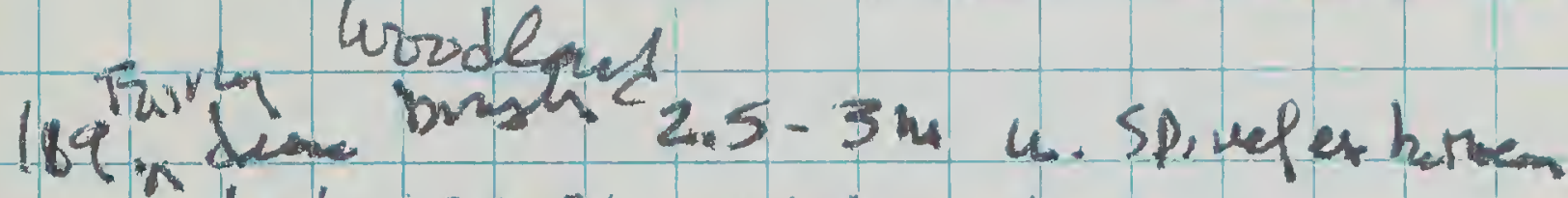
"Iha. T-boill, $2+$ Weatill

120

Vopen goum unde 6-74, dense us of $3 \mathrm{~m}$ 12 shows - nud shulas les - $O$

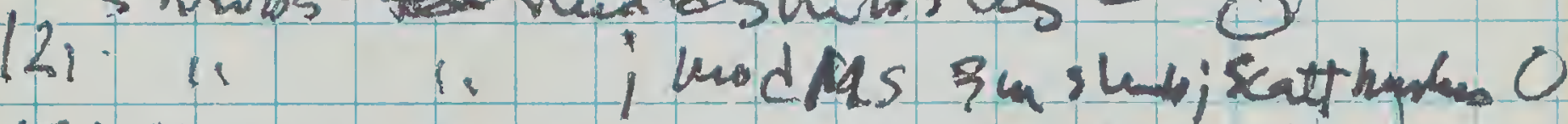

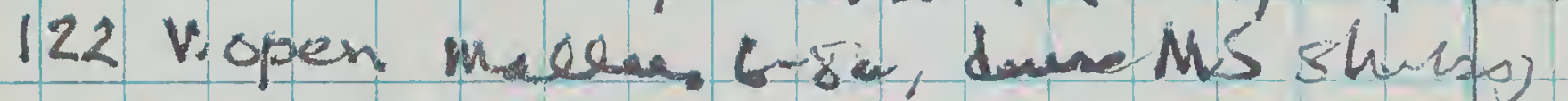

$$
\text { soct us slumb - o }
$$

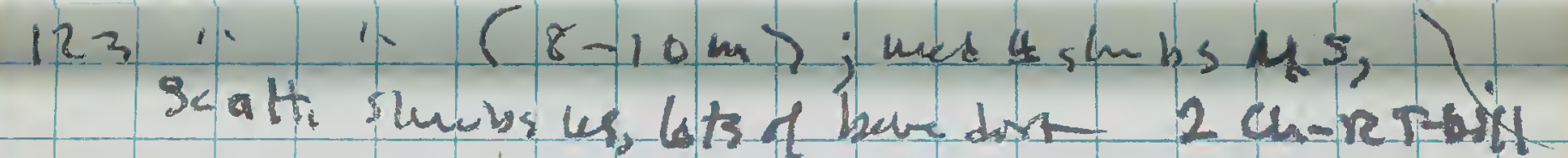
(turn N. tousand hig krag heve) $12: 1$ a pus Salm- Word. pu-nm; nod US of psendojupur (Melaleven?), sent US shoboso

I2s xpen Mulee $5-6 x_{\text {; }}$ sear us shobs,

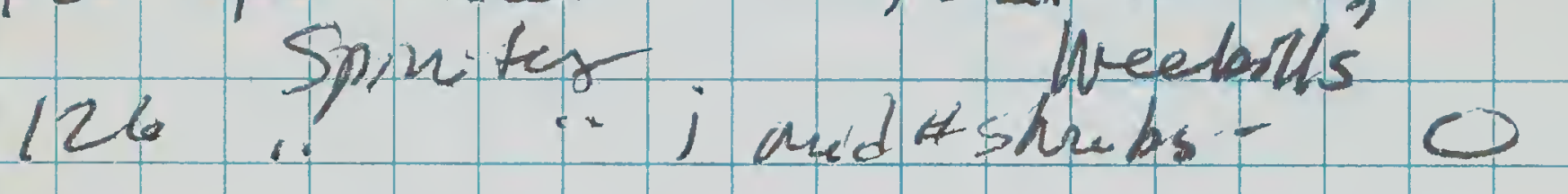
122

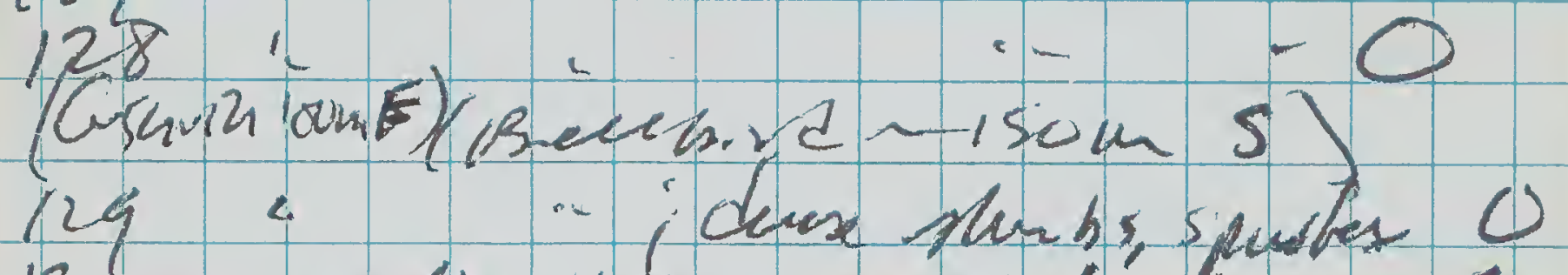

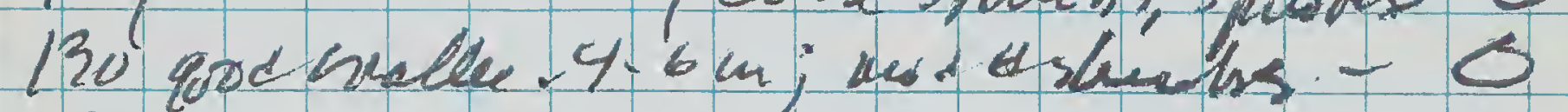
13

132 10 133 open qun uride. lom i mules shobs - 0

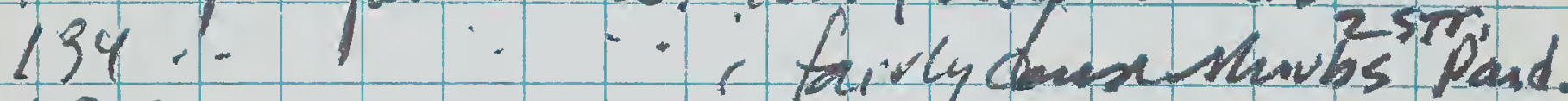

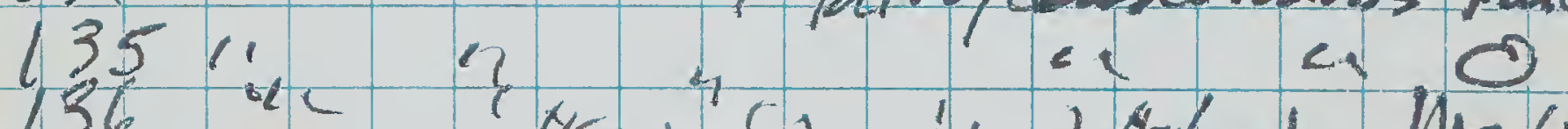

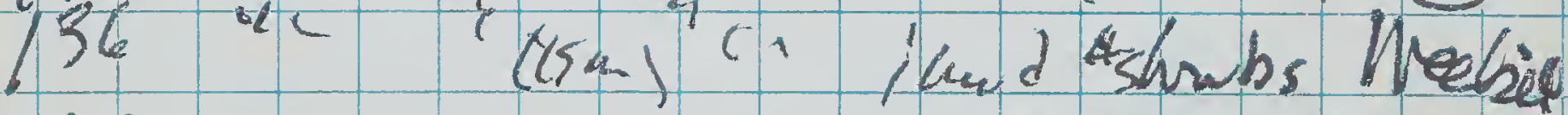
$137 "$ " " " sonphite, neelsiel $(38 \cdots+\cdots$

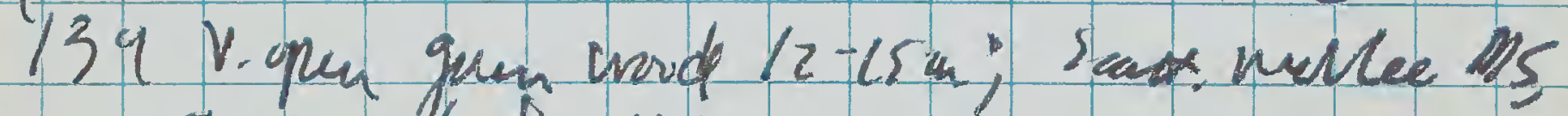
stad, surbsus - 0 (3) Cant E. Toward track wher 140 om is puhes) 
SReebra 1 Grsurna

13) 14

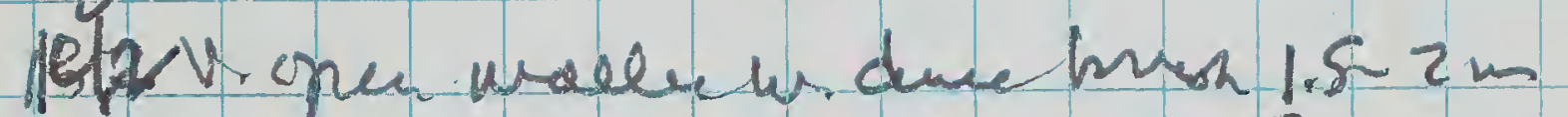

$1(13)$

2 Int T-bills

UCt 709 o

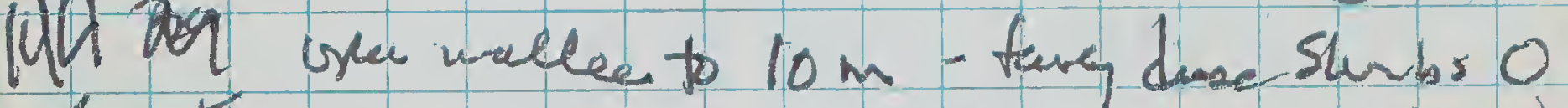
Whaobene buse a-3M - 0

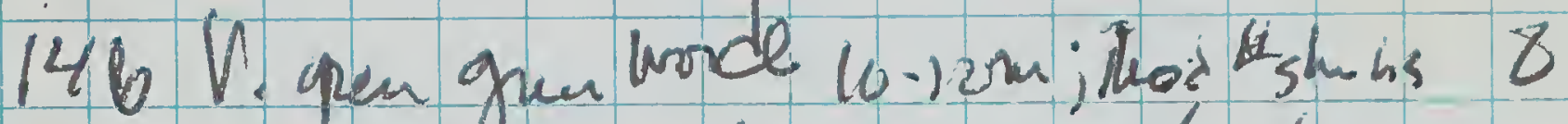

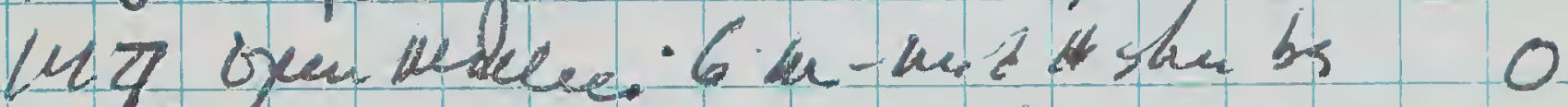

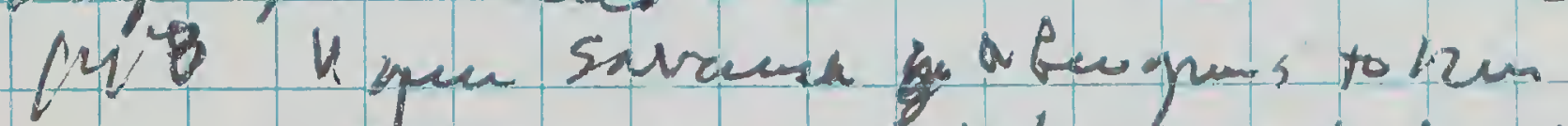

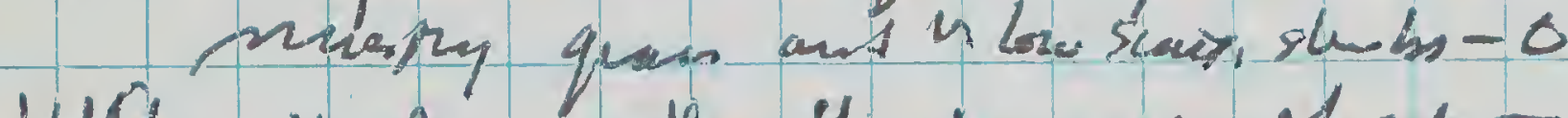

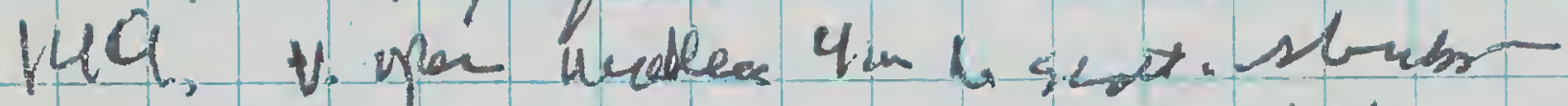

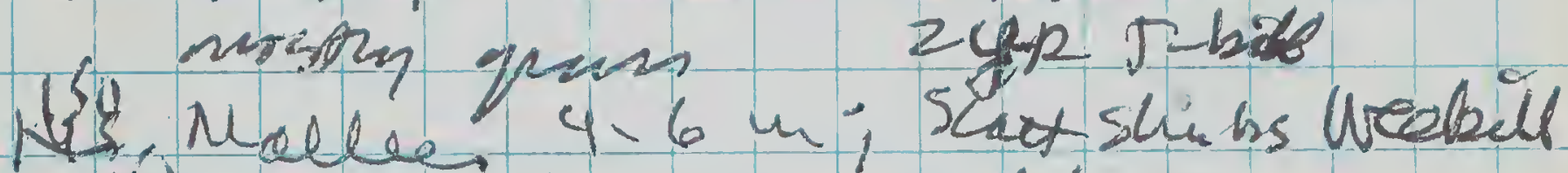

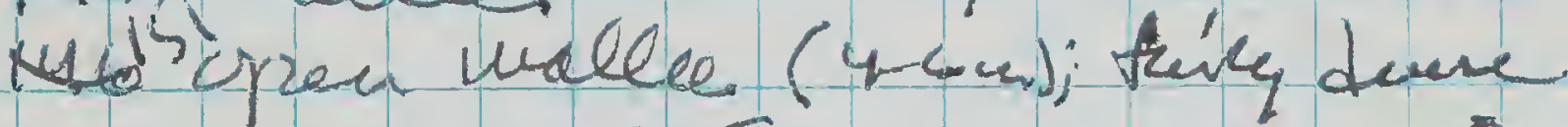
15 sumbs to $2.5 \mathrm{~m}$

17 F.derese bubs to $3 m$ 100 lis. $^{3}$

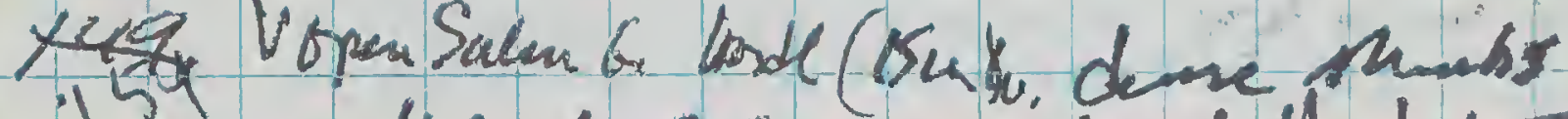
it in 45 to $2.5-3.0 \mathrm{~m}$ wedrill, InL.Tbill - Car lovm aura - stop

493.6 yereow hive 531.9 s.cois motel $13: 15$ leave at 15:30 for Kodya549 . $0_{7}$ 0 ) 550.8 Proted thriar + Katrel 557 last rande $N$ 567.1 puel of in dut traek hoading N. tom pared rd int unodland. 


\subsection{STOP}

$16: 10$. Stuit rack cosid liquta quand 15) open gam wode. 8-lon scat slubs

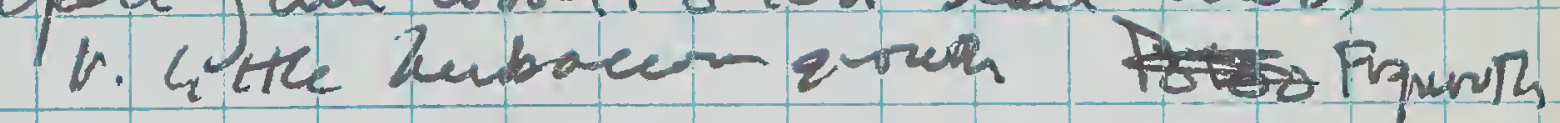

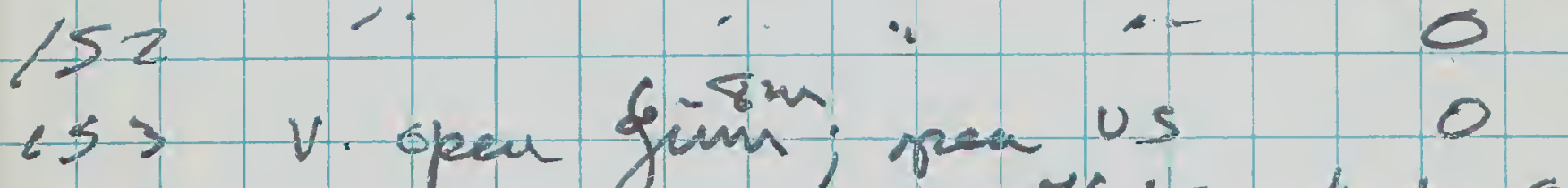

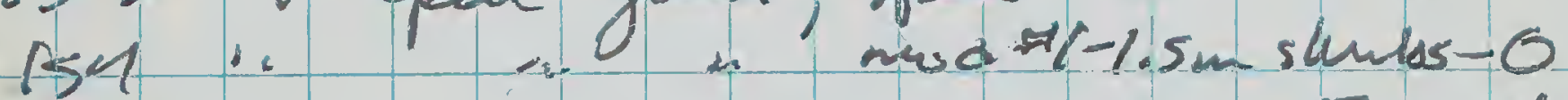

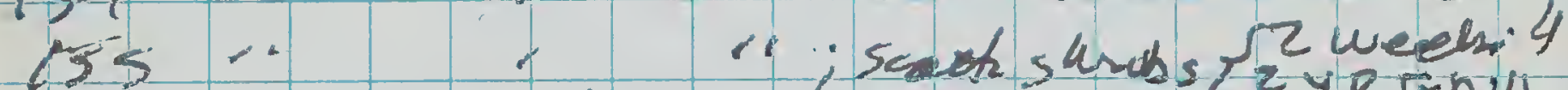

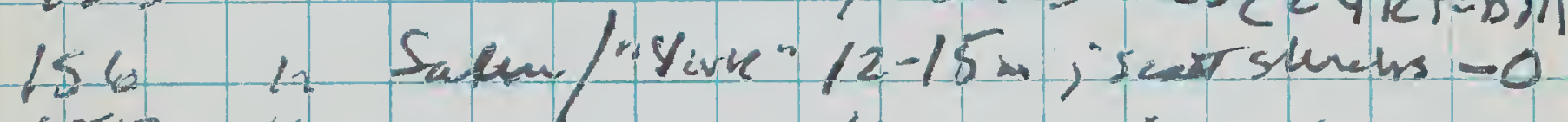

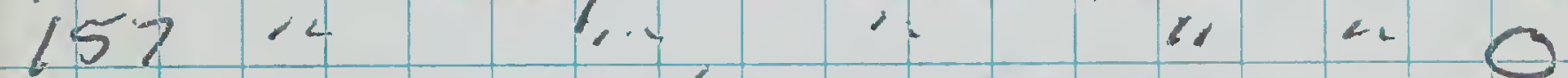

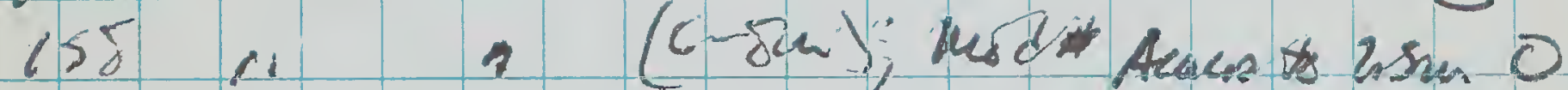
$159+\ldots .0$

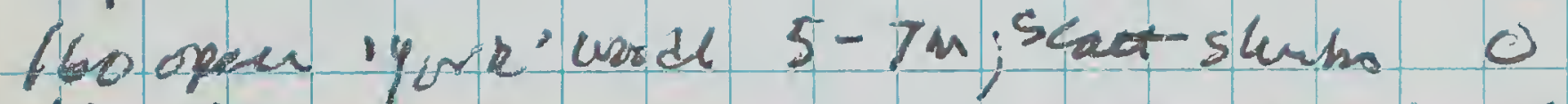

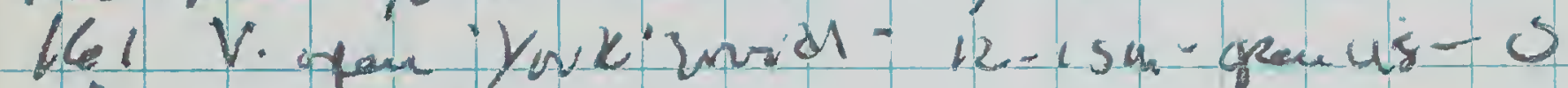

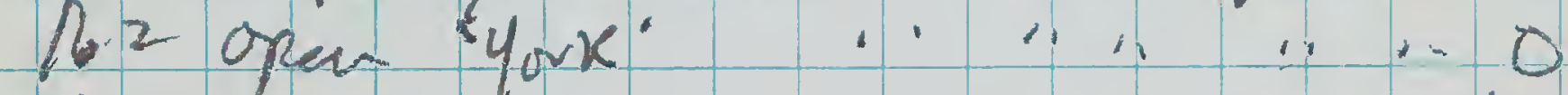
lu' i W Weatiy (Goshenk / $50 \mathrm{~m} N$.)

164 ... $\cdots 20$

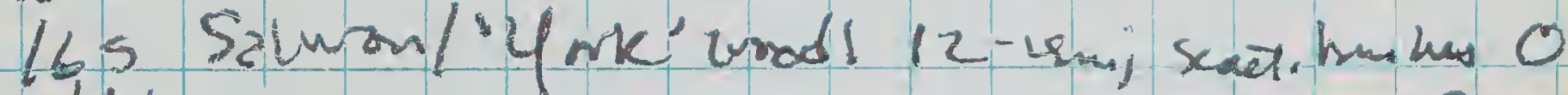
Vleb " ".

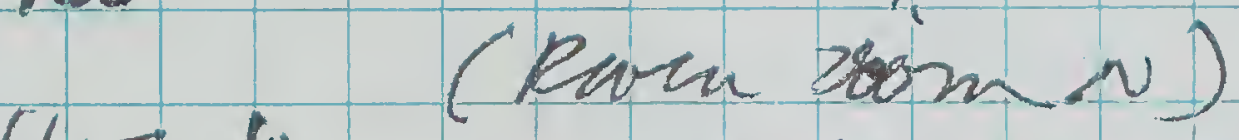

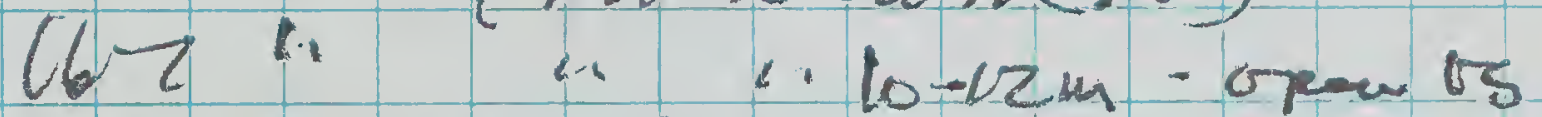
- R.C Rbin? ume res on cap Thea usung, Ithile, sud dif cole

168 in $\quad \because$ sactislembs - 0 16 opran salur unde $10-12 \mathrm{~m} ; 4 \quad \because--0$

$170 \quad 1^{\prime \prime}$

\section{(turn SnTh kere)}

4714 " $" \cdots$ jopenus -0

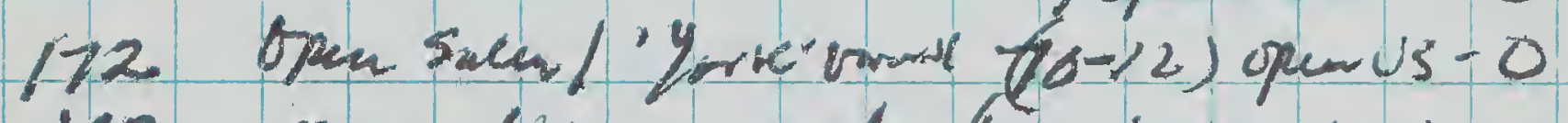

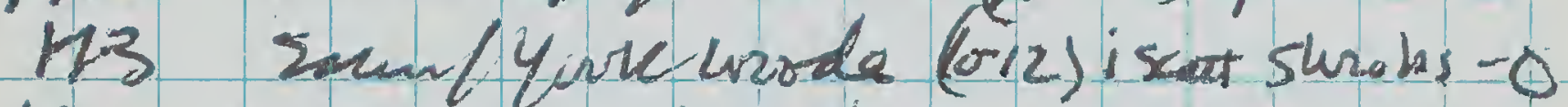

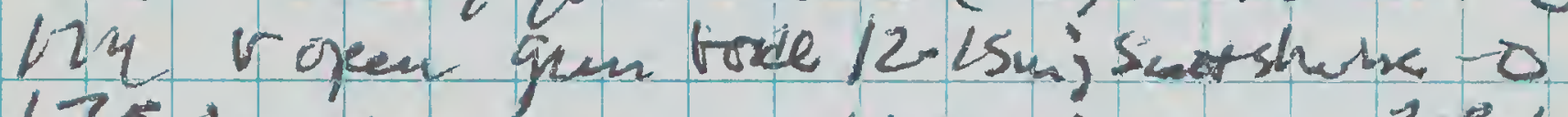
125 wopen qum urode $15 \mathrm{~m}$ inem us- 2 red

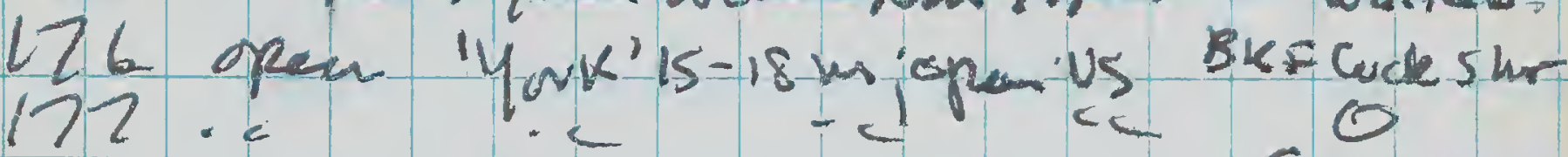

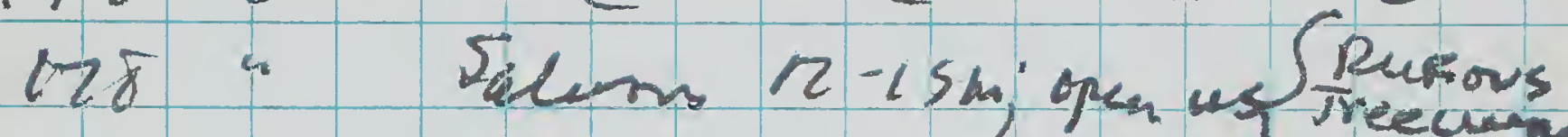

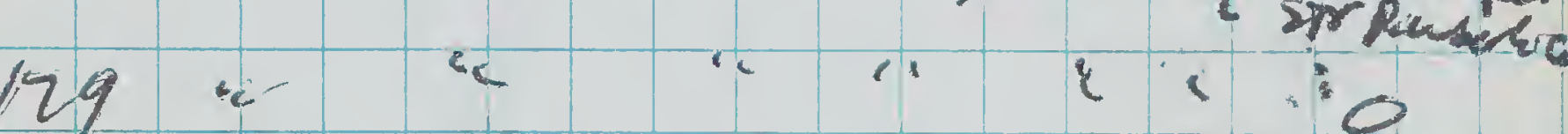

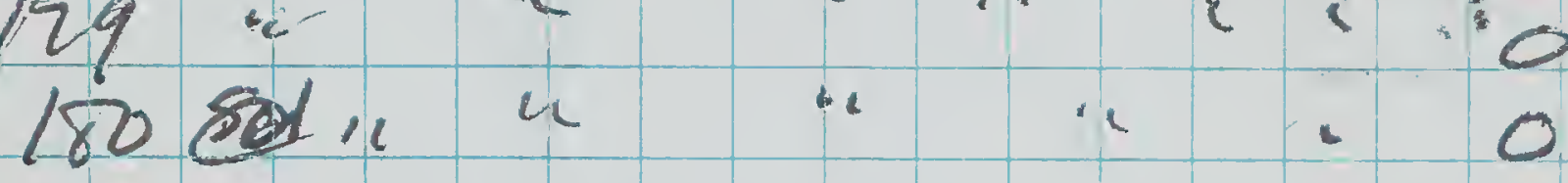

\section{(turn exst here)}

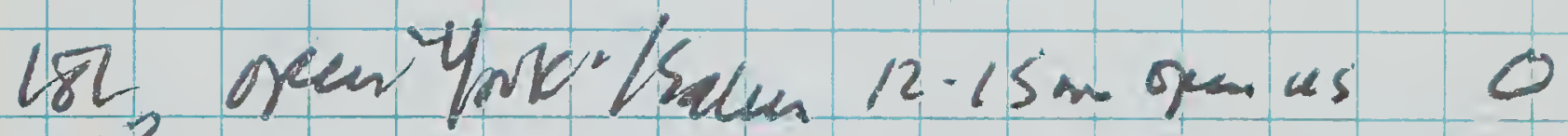

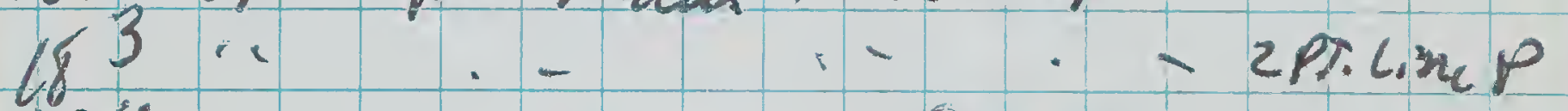

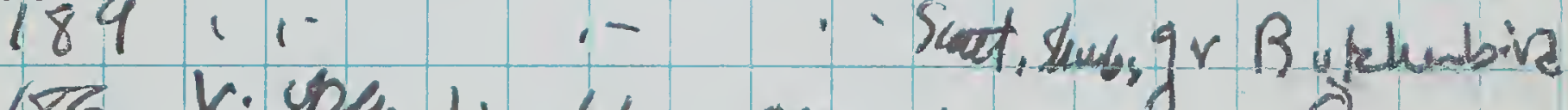
$483 \mathrm{~V} \cdot 4 \mathrm{em} 1.11$ gean us 186 open 'york' 187 188 Yank wodl.12-15m tom us (Batho (SOm N) 0 


\section{Bellewid 1 Gir sun rur-1}

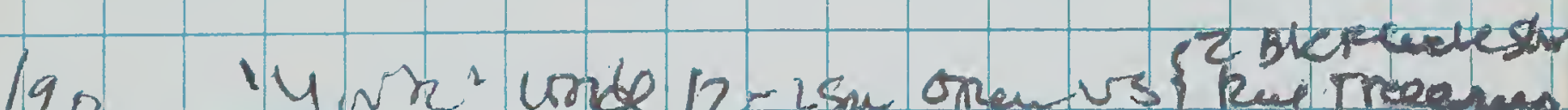
$q$ l open ic

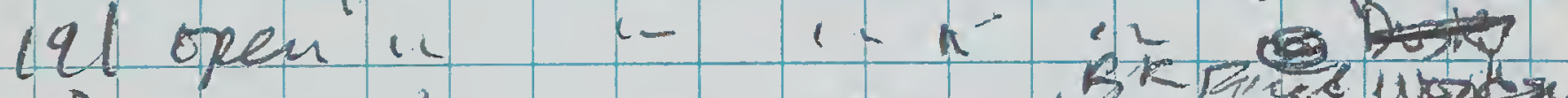

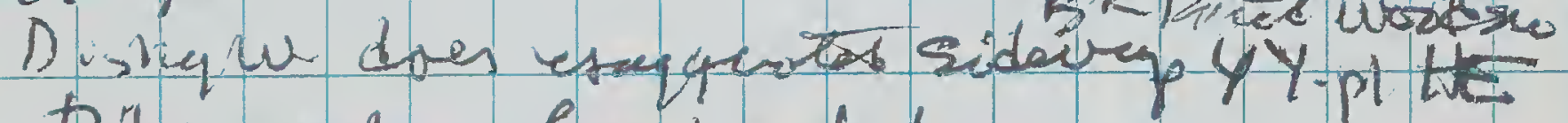
Dil uraqle when pached

$$
\text { Consurtin } 150 \mathrm{~s} \text { s) }
$$

192 hopen York 12 ism genus $143 \ldots 2 \ldots 200$ 194 open quiklsalua 12 -15mopenos 2 Wiske wrogtail, 2 yedPAE

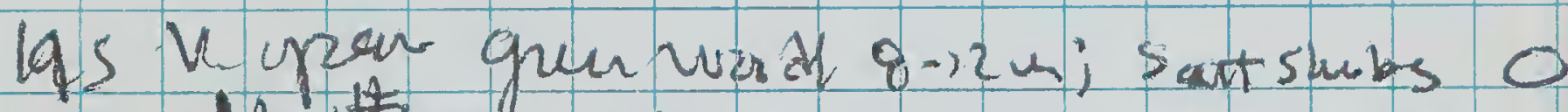
196 Med surubs $2-3$ m 2 weatiel $192 \mathrm{n} / \mathrm{n} \quad \mathrm{C}$ 198 Vopen guma 6-8m; scastshubs-0 199 open.

200 in quen 1. in Jachy huta

o Ther spp: Cunnawore

$$
\text { Ens? }
$$

$$
\text { cena, Ghw, mild }
$$

567.9 buk at pured od. S. choss if:00

Bivds in paddocks alvz rd to Koulyanototing

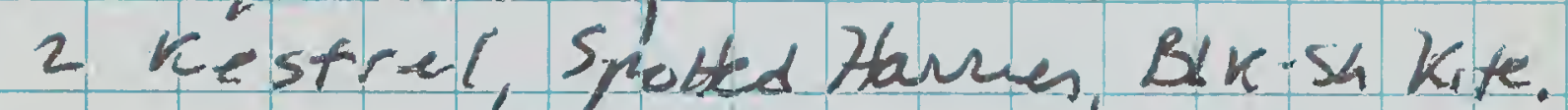
wany Galah, B. L, ne P, Wagpë, Raven, ow wested Bugren, Richad's Dipir.

4 Nor. Ien.re S. cross at 06:10 sumy, calen, cort

2603.0

reed to 90 60-6s km w. To cradlade w. I Corrabin

2665 
YNin, OGiso 2662.0

STa. 1 exteusive area of Kurngan avong buy W. of Bodillin

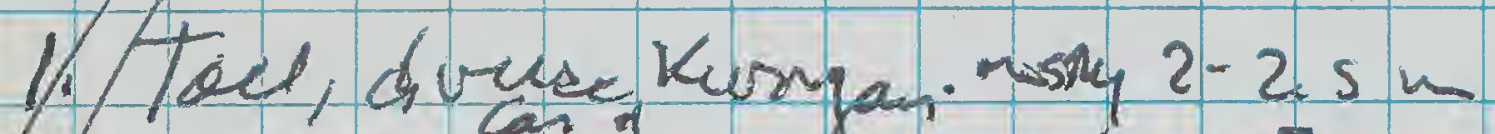

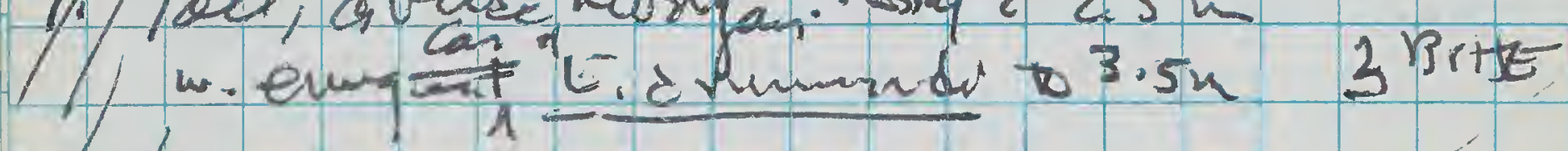

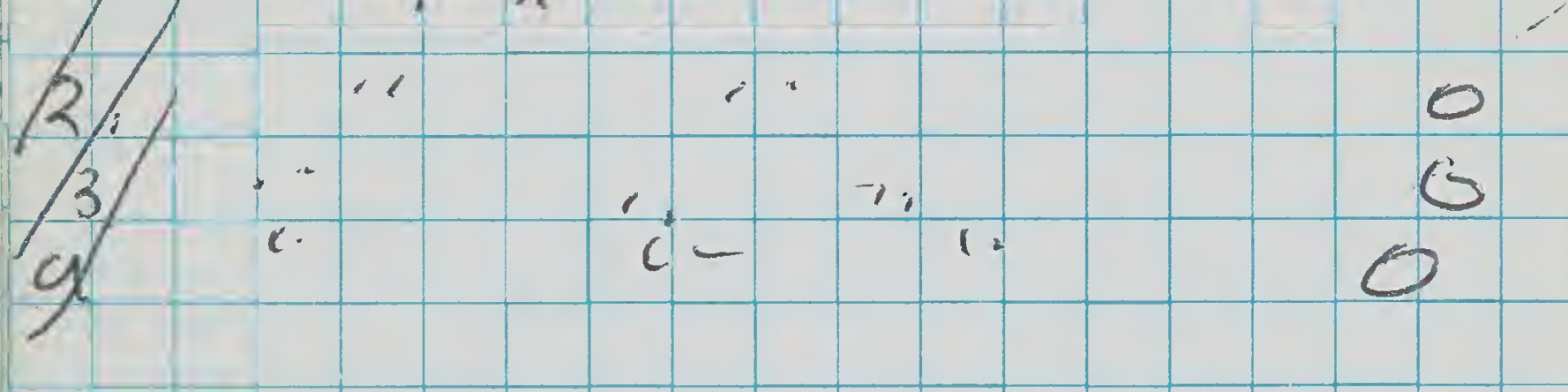

ltumed ost nhe Lewt a nama

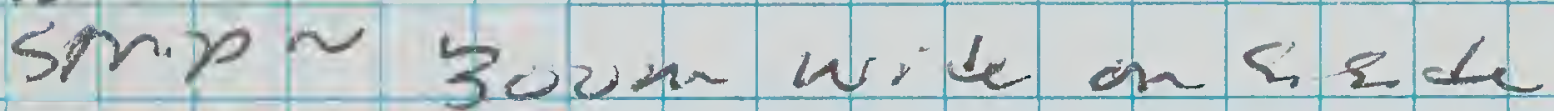
of $\operatorname{lng}(3$

Ste 2 2L70.4 (at RR bridge E. Carrabin

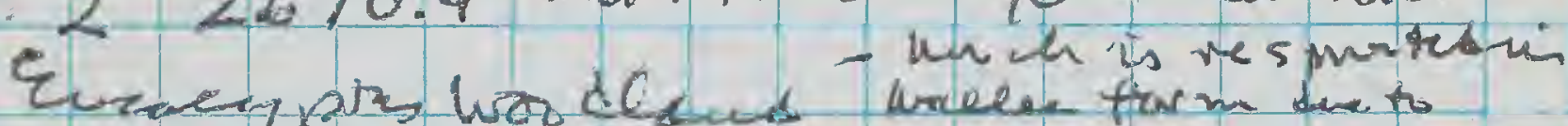
wreles fiarm due to MS ureen.

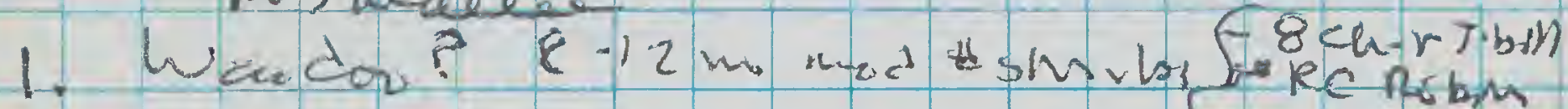
Ev urehil

2 Mailes 8-10ar saxt. Shuks. 0 3 … $\ldots$ 412 modthlantos CRC Romi

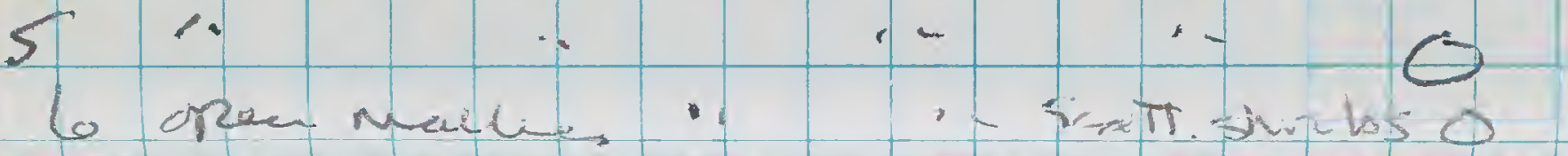
F $\cdots \quad$ i. $\quad$ opan 450 a.". - . weetias 10 Maver G-8m; modrshions 0 it.. 8tom; i" resbills $12 \ldots \ldots$

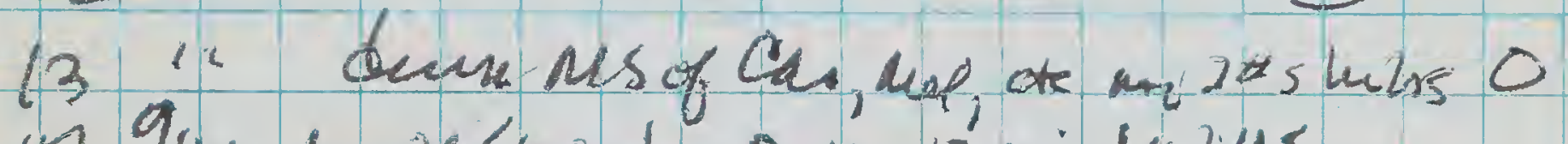

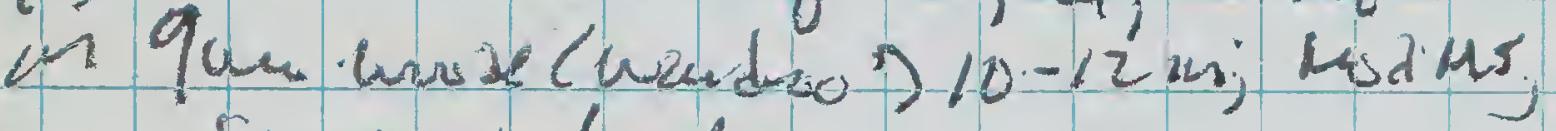
slactuat shubs o

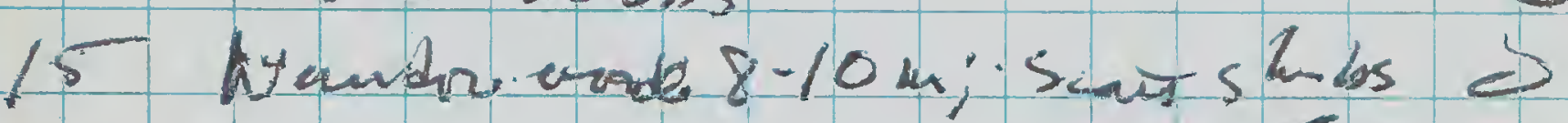

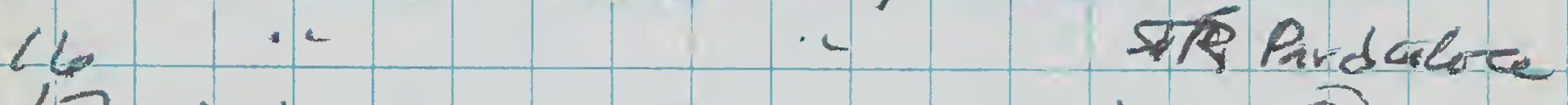
17

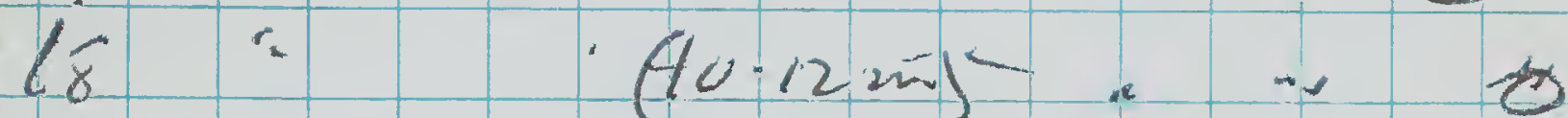
lq Sulmar lo-12u; Ms Mel (Psudojumpu), Staw skent b?

o cpen Sufum 8.10 m: scaut ms, as 0 $21 \cdots$ inmodus, Ms 0 ( had 5. towad thing her)

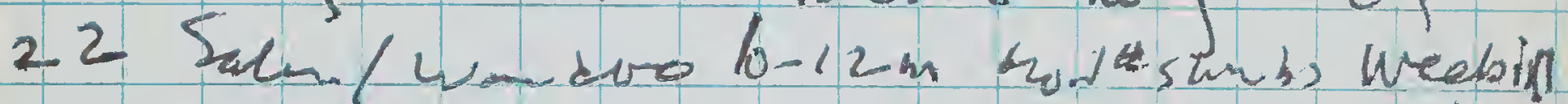
23 Wandor :- 1 . Sutt. Shurbs 0 24 Mallee 6th; seat shubs 0

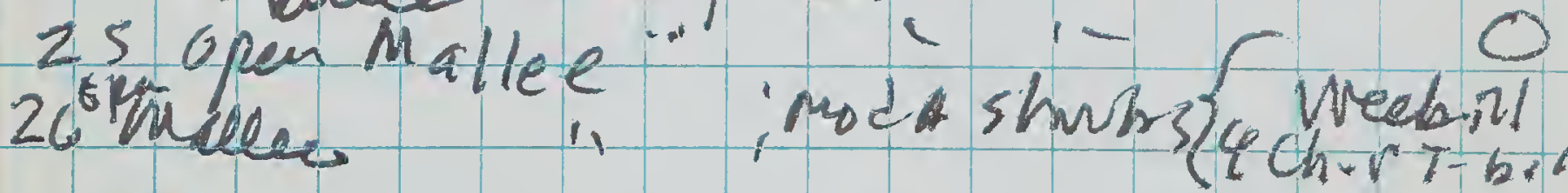
27 mullee ; wove shorbs $28 \cdots \quad 1$. 


\section{Now cannt}

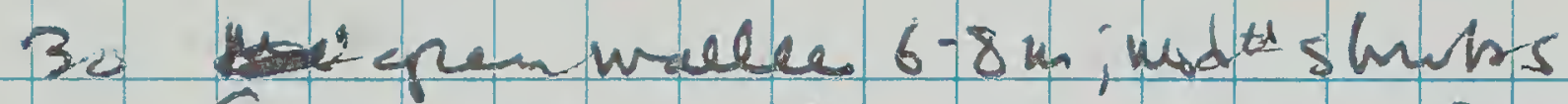
Cacerpiter sp feew Murghtres - Bringos? Yes- I laos daer it priched I

31 V. upen wallem $5.6 \mathrm{mi}$ seat as wheard be (Modue wh Fowa-viste)

32 mallee $4-6 \mathrm{~m}$ sat shares weebils Carshan isom

334

34

35 Made 5 - arshrin. hod th shuts

36 Mallee 6-8 4 ; wid dane us - 5

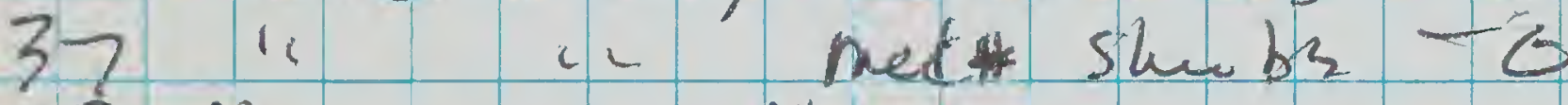

3811 in Neabills

39 epen malle we ferl des hashes to $3 \mathrm{~m}$ Br neaded HE

(olue colued z'sucke dove intz burrow)

20 Vigan ge sutu und /2-15m; faicy

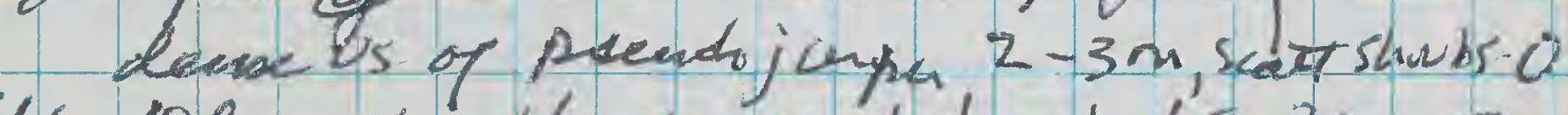
te open scimbland nad denstyl.5-2m 0 Trshorin!

Q2. 1 spenquen arodl 8-com; wod \# Tare

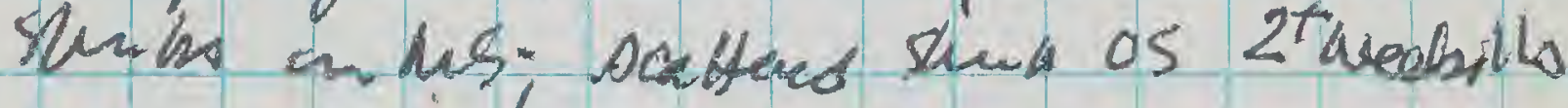

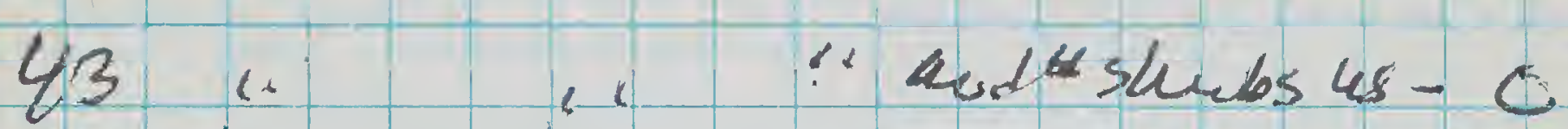

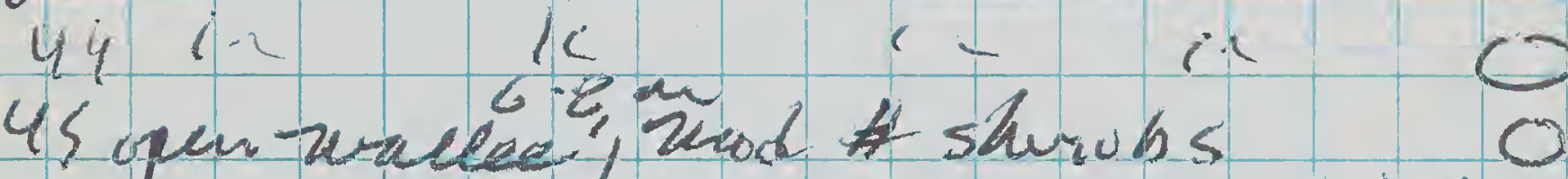

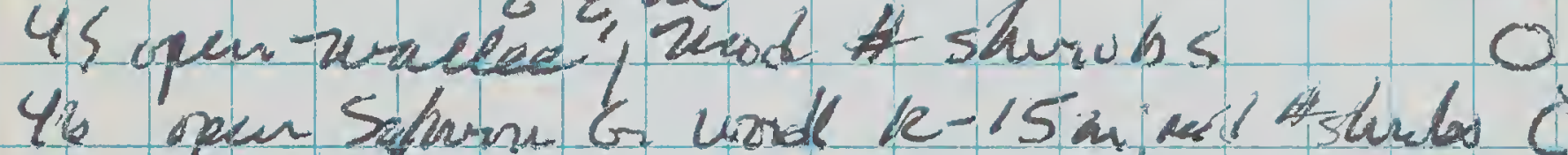
47 Silnem enid, 8-204; inad Ms, and is 48 salm 10-12n; madMs opan US-O

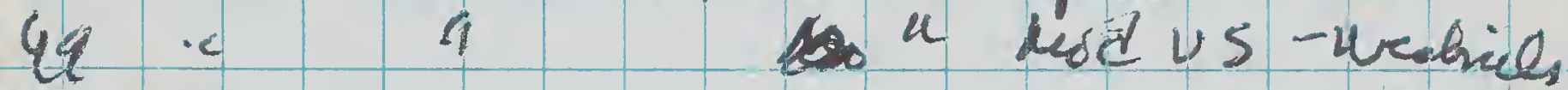
So $k^{2} \operatorname{gren} u s \quad 0$

$$
(8: 455 x p)
$$

Seen kut nit in Thamest Galah (ipr), Ravens Nore: No PT. Liñ. Parroß heve - odd!

\section{4}

$5 T a .3$ adpocent heath ToN,

si dreuse kurnan bo grevillen, Horken $83 \mathrm{~m}$ qrshrth- 2 s 5 . Pard. Ref, whistler, BrHE

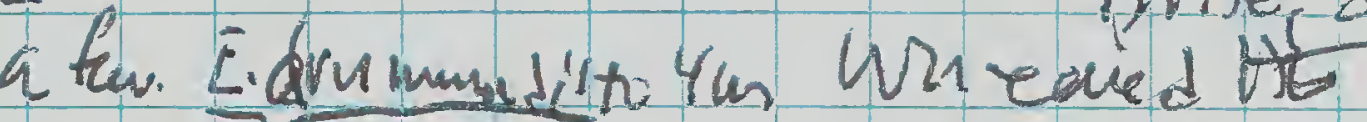


54 duse div. Vuray $2-3 \mathrm{~m}$ v. Sat. Edercumondit To $-5.6 \mathrm{~m}$

55. Avly deure Kurnzan 1-2ur; Seat:

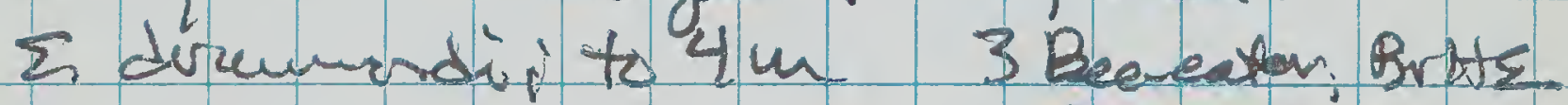
$56 \ldots$ abellains

57 tellen durkengan (Inke 54) bat Erdr. -5 Barken, yeur - plumed tE

58. 1.Ken 4502 bec-eoters (IAff tam 55) Ces? Redturoat of

Hoided

Robm Fouded hemi $14 \sin 5$ whiten

puth

59 toen Rerga (like \# 54) Brit) $60 \cdots$ (Moedrcaliondi) Bute

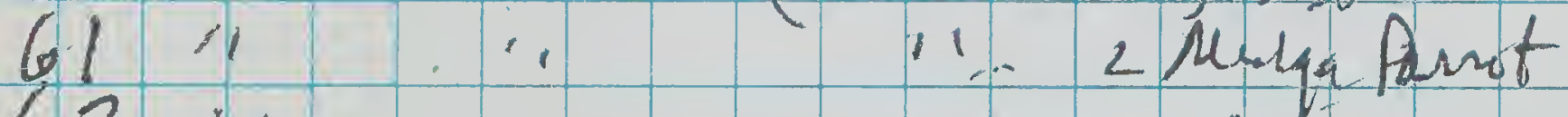
$62 \%$

63 Castqrevika keatu 2-3m:

$64 \mathrm{ri}^{\circ}$ 0 65 a $^{2 i}$ in $67 \%$ ragle orited)

orsurtin 11 fow wh! Beeline

68 Dirusekrorgan 1-2.5 m

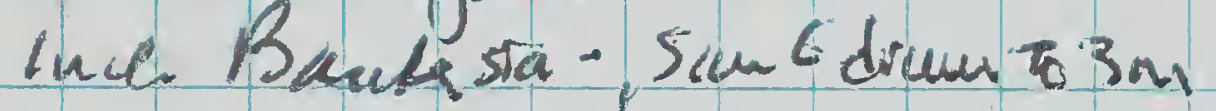

64 -

10

21 Teedr Konrges 2-2.5 w Es semuonds to $4-5 \mathrm{~m}$

$72 \cdots$ C. B W BE 13 (maypes meizad) Corss io E side of ter pracks and work back S. To Car.

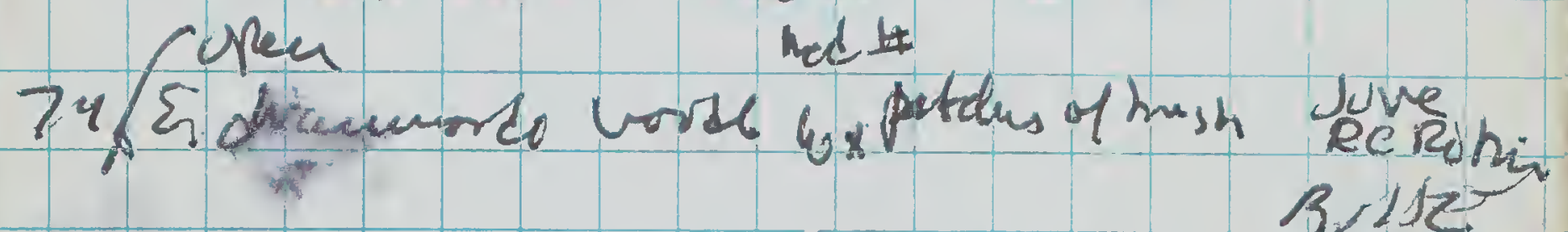
Th a th Thillsp.ibitle

26 deveme kurong an 2,2,5 is w. Sceot 1 E. dirumma! to 4-su 0

\begin{tabular}{|c|c|c|c|c|}
\hline $77 \quad 1$. & १ & & $c$ & 0 \\
\hline 7811 & $t$ & & 1/ & $\delta$ \\
\hline 19 h & Cl & & 1, & 0 \\
\hline 11 & (1) & & h & 8 \\
\hline 81 & $\therefore 1$ & 2 & & Redruvat \\
\hline 824 & & 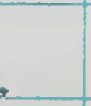 & & $2 \sin 4 \mathrm{He}$ \\
\hline
\end{tabular}




\section{Nov-coutd}

83 diverekongar 2-2.5m; scaet E. dorunantis

84

86 jien 4 neaters

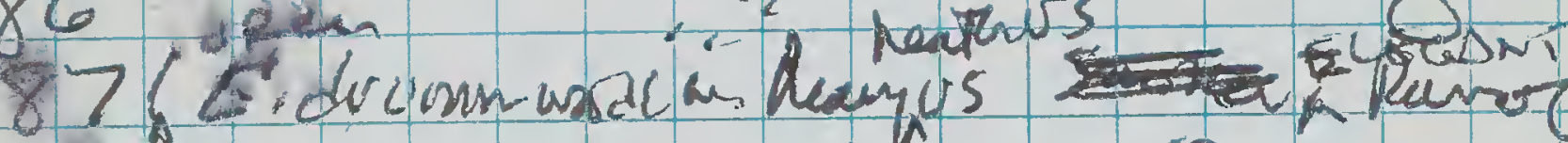

$88 \cdots+140$ 89 " U 2 S J 90 disuen Vurzan 2-2us in w

scact Ĺ decomisne to su 0 Q1 Acalcir-dhearated warkidaí 2-3m 0

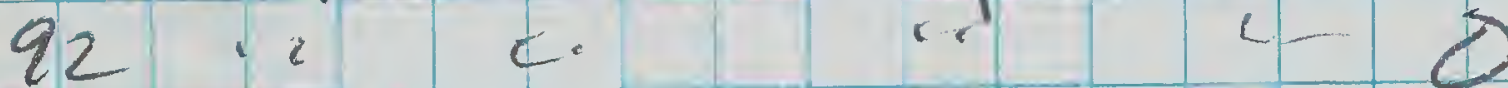

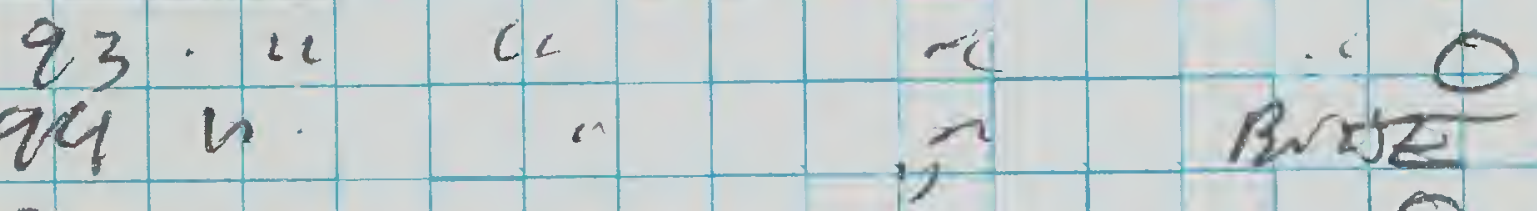
$95 \cdots .10$

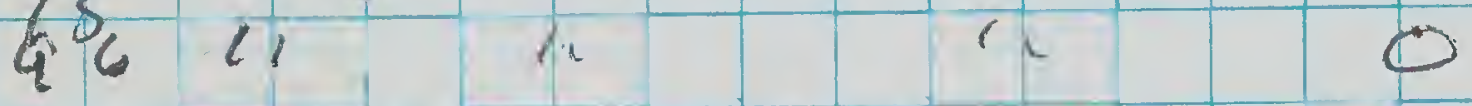
(wiss kath to E sidef trak)

47 diventeurraiz-2.5 nu. Edu to $5-6 \mathrm{~m}$ BNHE 28 . 5 a 0 \{9 Rrte 100 Sut 4 , 2 उURE 2671.2

END de 10:30

lather d wen R

2673.1 tum back - lears still cutiries

2675.45 . end of heath- hadew w wovlland

burds seen, hat wet Gunted Galali, Ricli. Prpit Litte Equ BK-F Woodsarallow. Kávea Mogpie

2676.0 lury - had W 79.5 end hertue vel. qas Reecenburin $37 l 2200$ 192780.4 hoon

Auvokoppin west cut ker transect is 


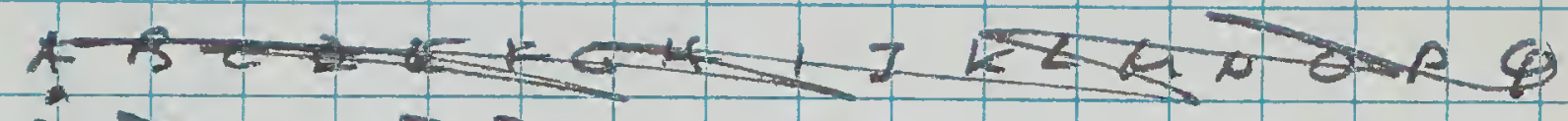

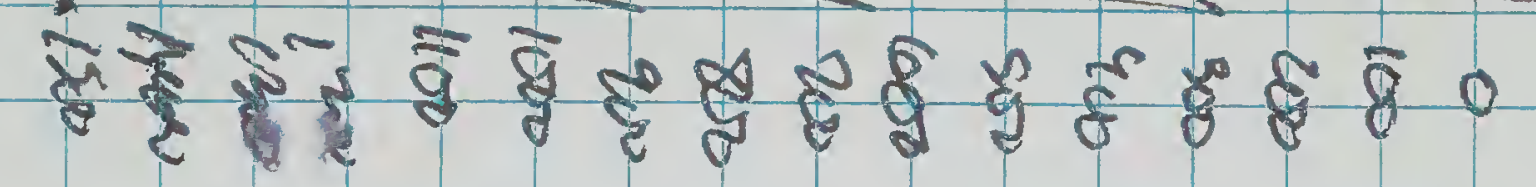

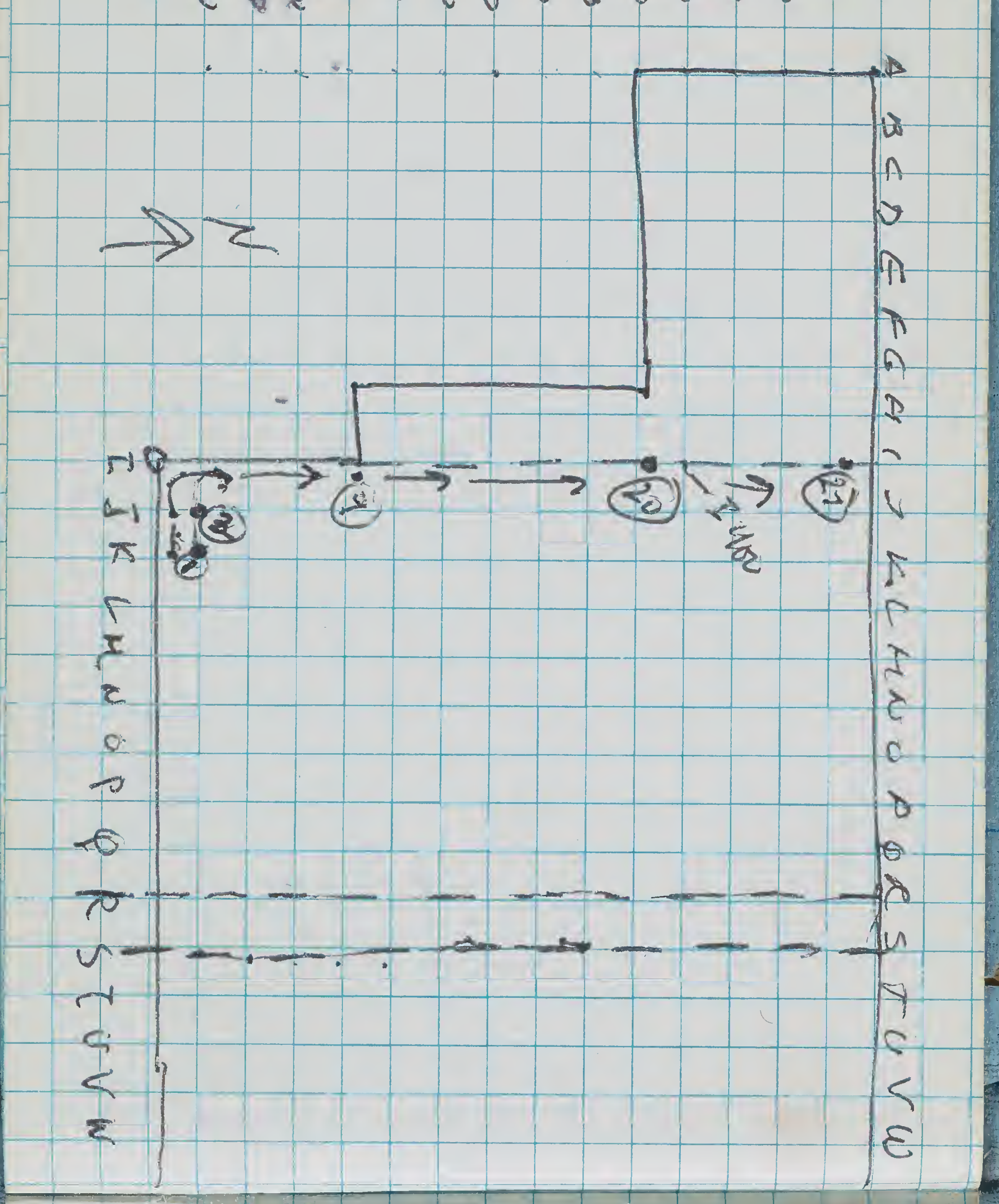

rene

ल 4

4 aver-anto

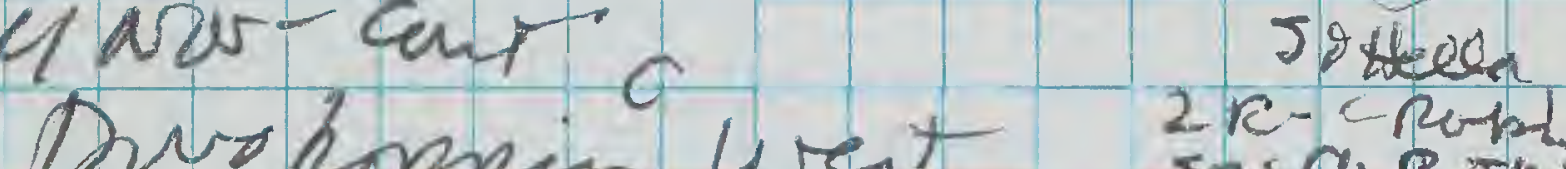
somf at If (100 $\mathrm{m} \mathrm{s}^{\circ}$ ) werbs

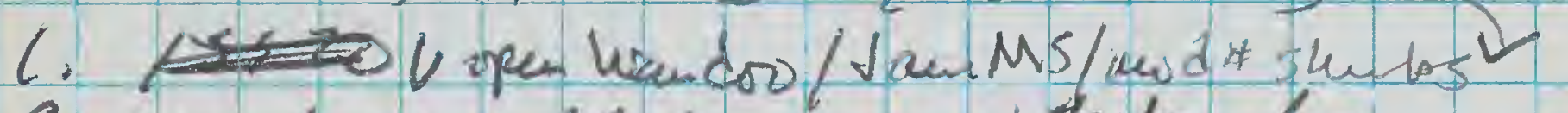

2. Waudor woride 12-15 mimid shabo SA. pudalat, weelonit. Py, Wrentter? 3 he 1 BKFCukshine

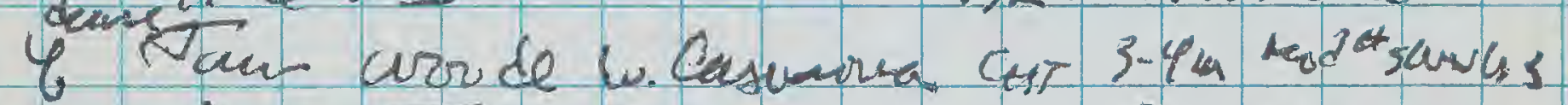
J. 5 wredill

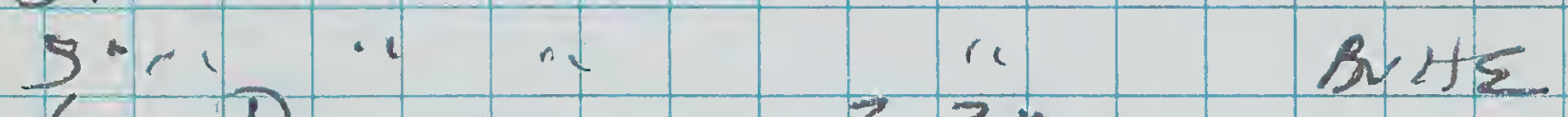

6. Dewe Cosuarina $2-3 m$ suttiond youk gre 0 2 Dane CalJam wind 3n BrHE

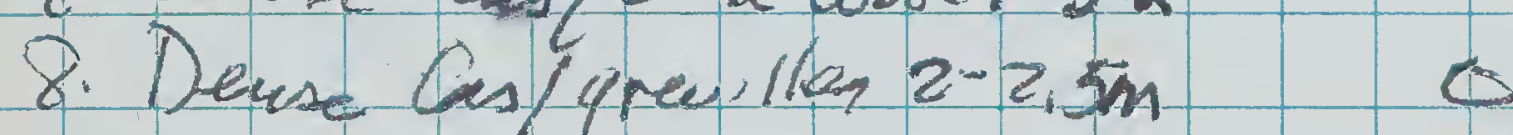

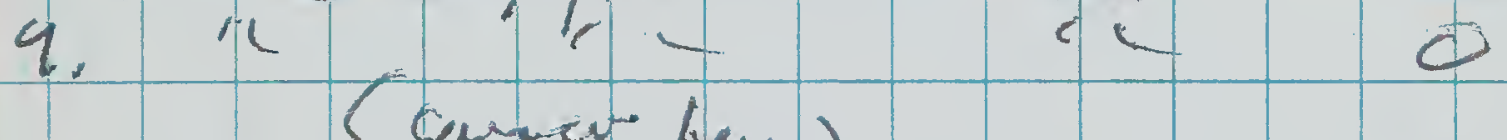
10... (canewhen $\ldots$

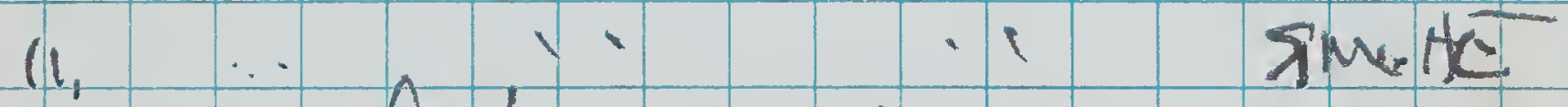
12 Deur Caslqnew, ete. 2-2,5m 0 ( $(10 \mathrm{sur}$ tha $150 \mathrm{~m}$ SE)

3. Vopen Yook $5 \mathrm{~m}$ w. deme as of Cos/Ene, $2 \mathrm{mo}$ 14 " ' is york wod $6 \mathrm{~m}$ i deuse os of Caslaver $2 \mathrm{~m}=0$ crrequthara 


$$
\text { Rupur } 11 \text { Grswtan } 1
$$

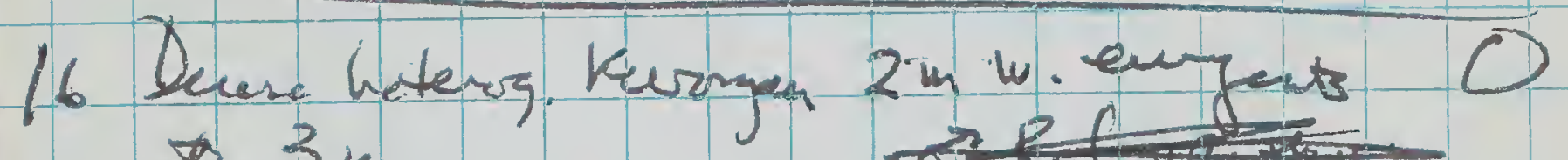
17 to $3 \mathrm{~m}$

(5) $(5-6 \mathrm{~m})$ " $24 \mathrm{mat})$

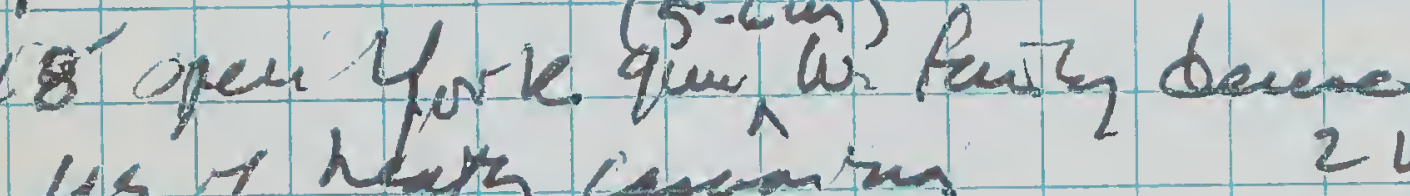

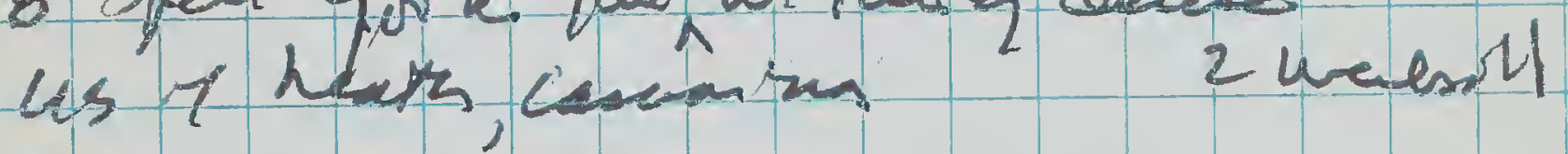

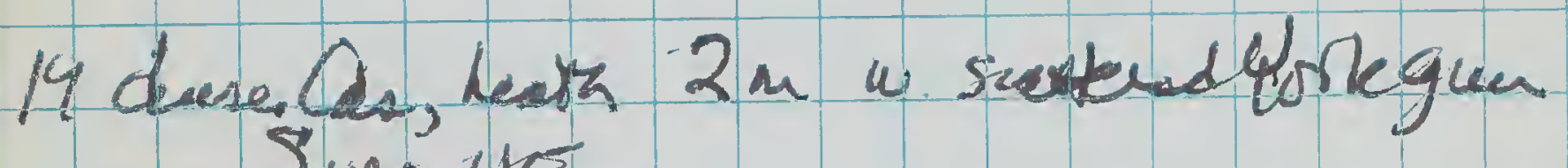
Sing He

$$
20.4
$$

$$
\text { in "wa-enadte }
$$

Cand re-ectionst caven

4 dense Cas, laith 2-2.5m

○

$22 "$ Brites

(Fyoo in Tainitureurl)

23

24 i

$\because$ Bute

251 " (Sewath York Jumpsm) 0

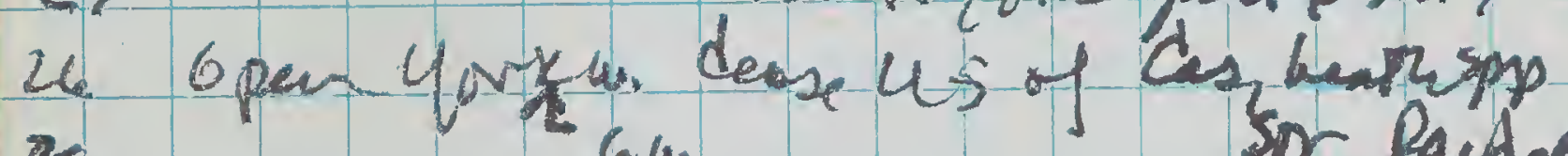
sor Paidoste 27 i

$\therefore 0$

(aloon fr. N edae herel

28 Yovk uerde $(6-8 m)$; modustus O EDGE

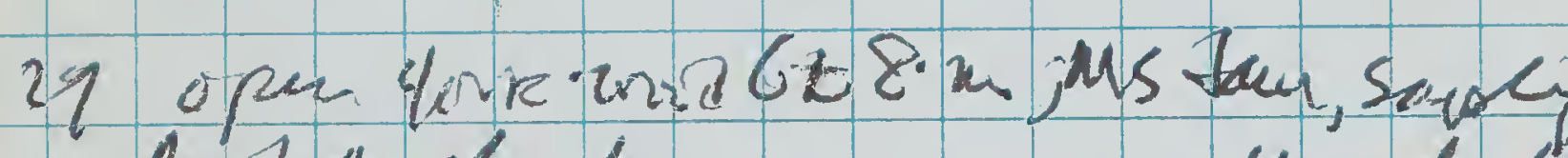
nod \# shrobs weelnts

3 4ork/Wandor wride 10-12m; F. dene is 0

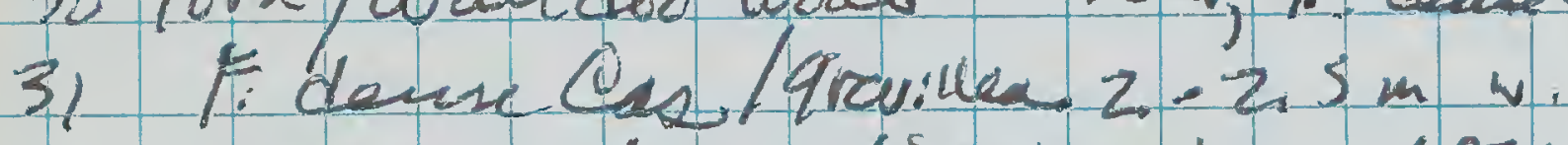

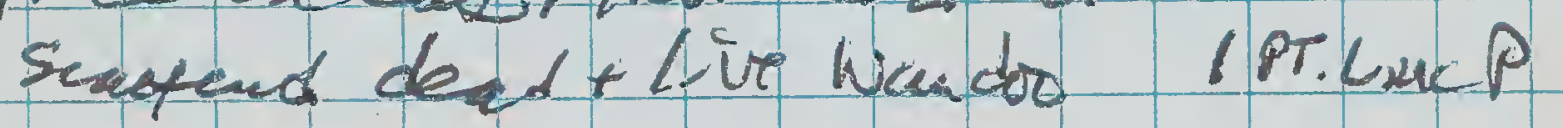

$\longrightarrow$ Fiscoes? $\longrightarrow$ ins? $B E-$ upale

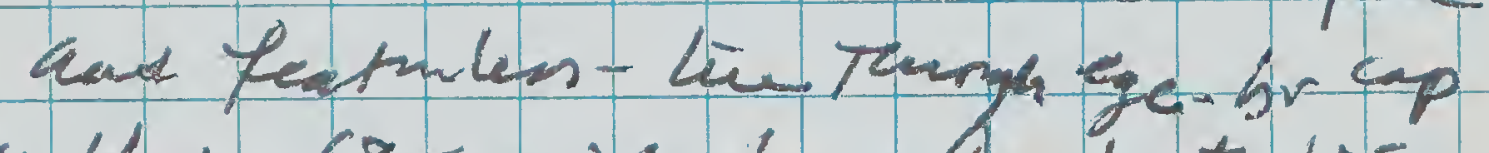

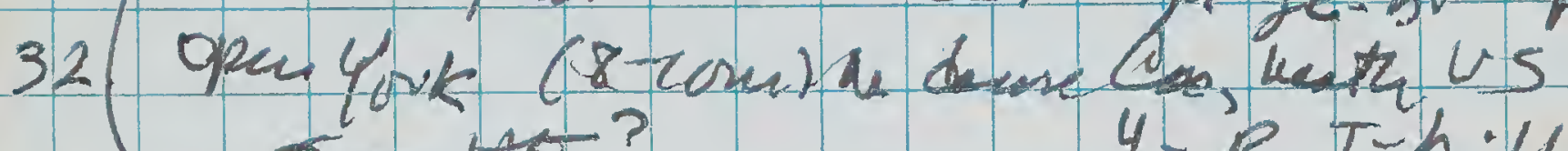
33 SIn $A$ ES?

4-R $T-b ; 4$

(skip rext sectim ol etgeme last week) " 5 "line at $N$

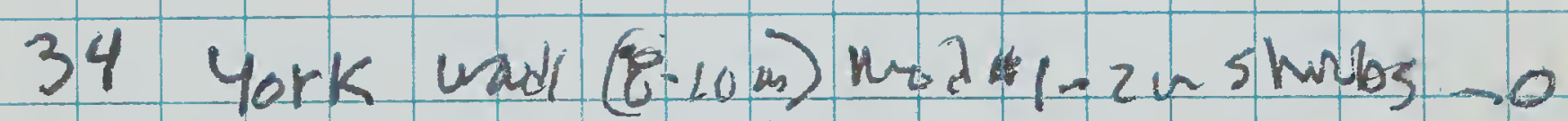

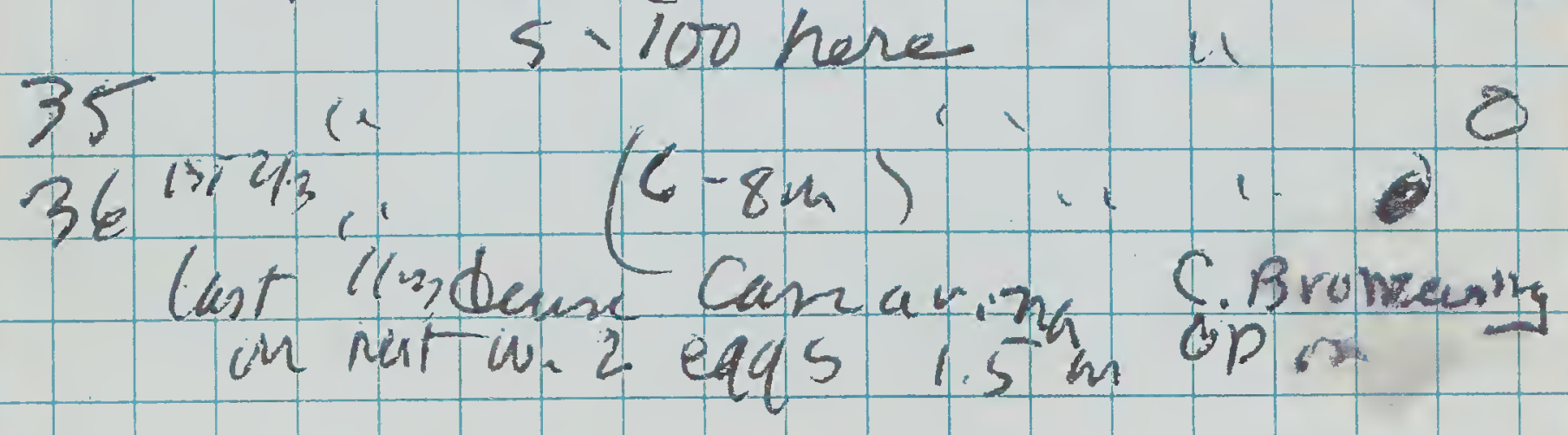

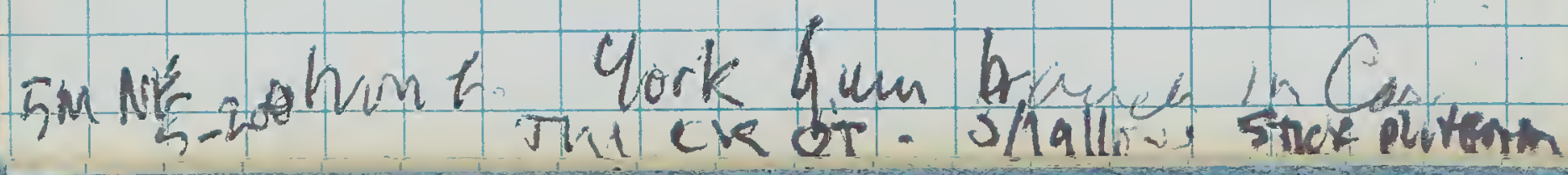


37 dense Casuar.m 2.3m

38 dene $(S-300)$

39 Cas/Melaleva / greulleg $2 \mathrm{~m}$

40 (s-40)

42

$42 \div(5-520)$

$43 n$ " $" 40$ P. Lie P. $(p)$

0 0 0

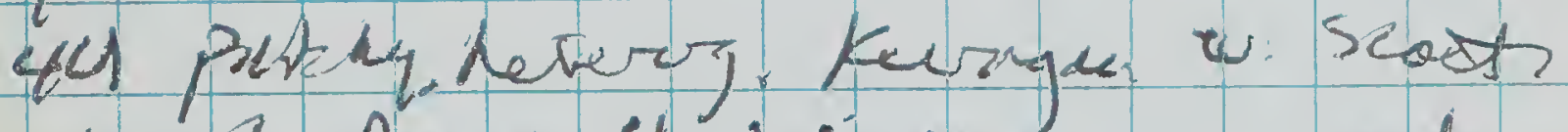
urode Rearto 4 migheath to $2 u+$ sedae cloup botfe $(5-600)$

$45, \cdots, 11$

46 a $(s-700)$

47 i. (Noworsinar) 0 $48 \% \quad$ (s 1000 (civody pear pras.) 0 $49 \ldots(3800) \ldots, \ldots$ so i. B B Prts $21 .(5400) \ldots$ Butte $52 \cdots \cdots+1-0$ $(-\infty, 2)$

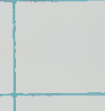




\section{$6 \bar{s}$}

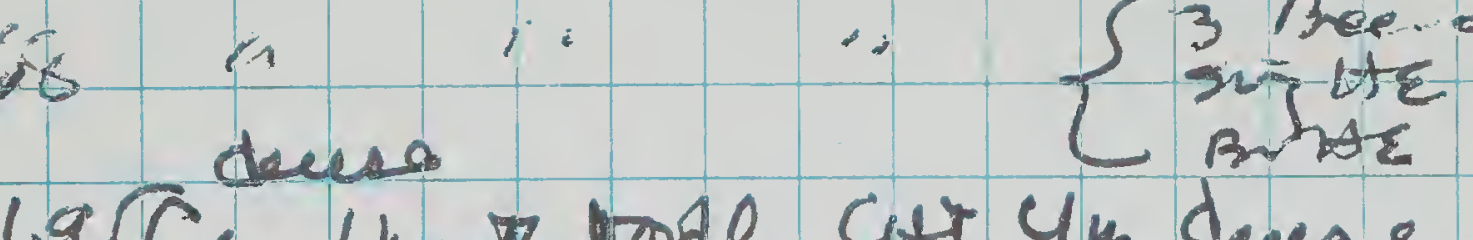

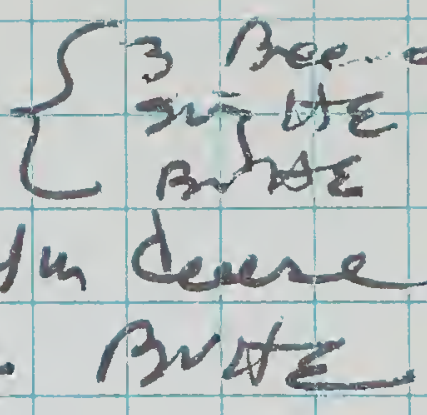

\section{$20 \div 12$}

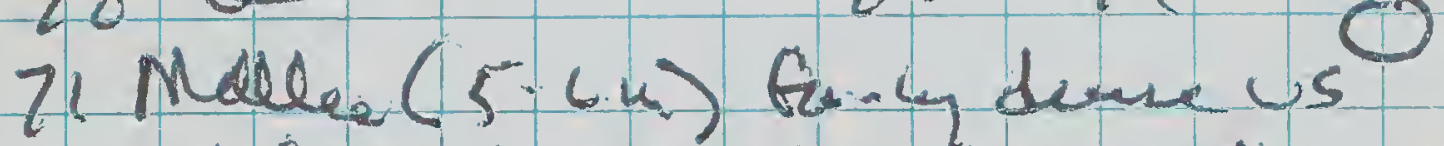

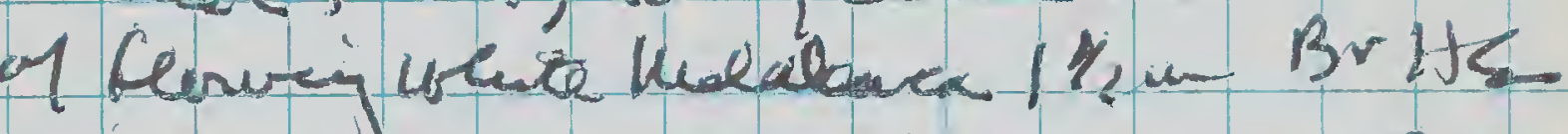
22 a

731 Weeb: 4

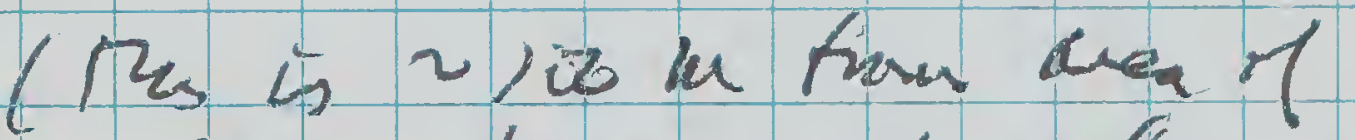
corde on heak's site of tear, $x$ cent N. it trade)

INTERIOR - nevy prock

74 qrod Malle urad i scaotssumbsus 0 $75^{5}$ dause Casarialara. Sunb $3 m$

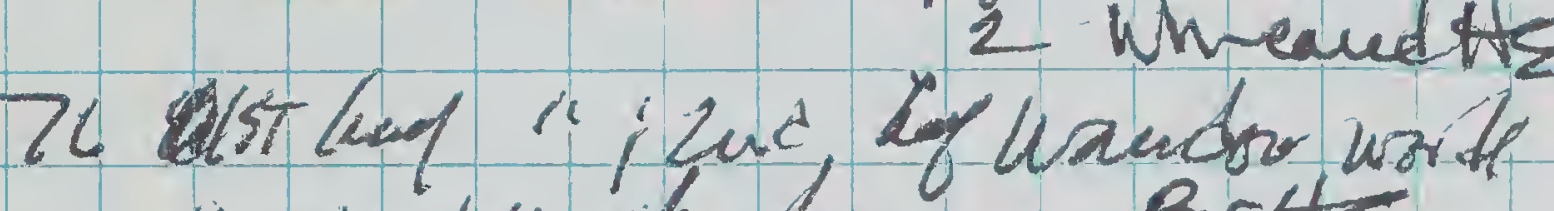
w. and a shmber Brote

\section{(STRP) $17: 30$}

Sorv.

WEZD3- 6:15 Clear, Calm, r.warm

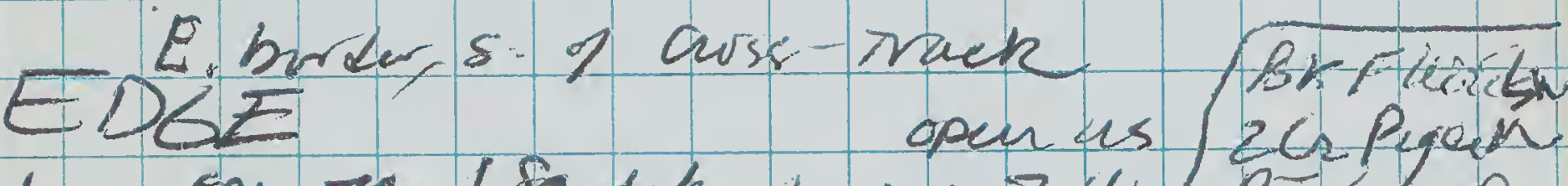

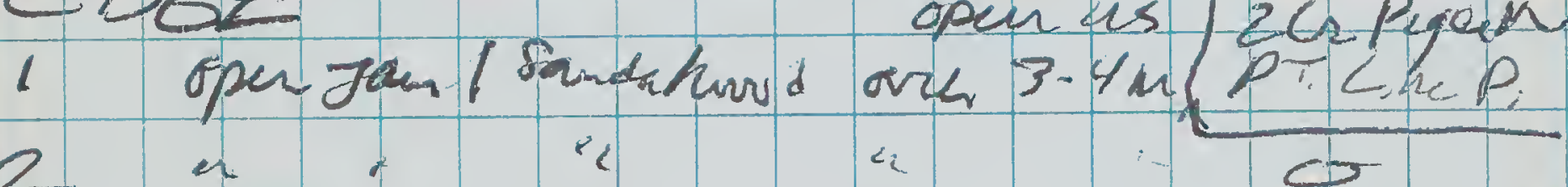
3 1 E) 3 r. binde

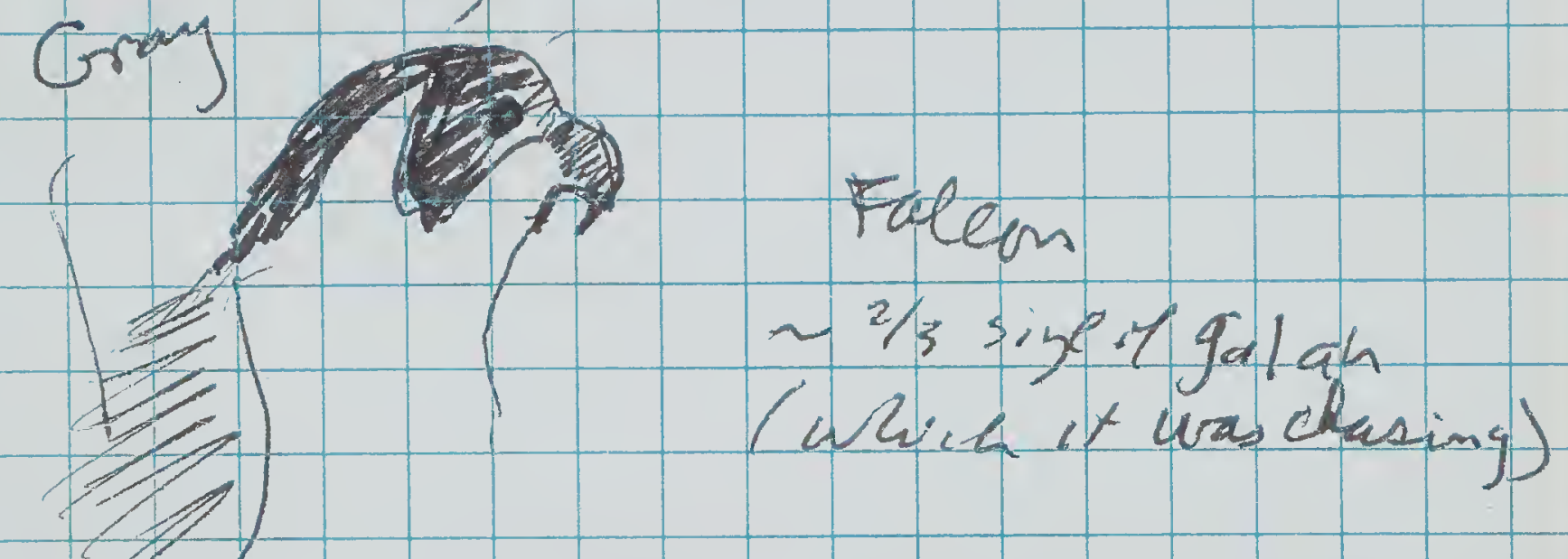

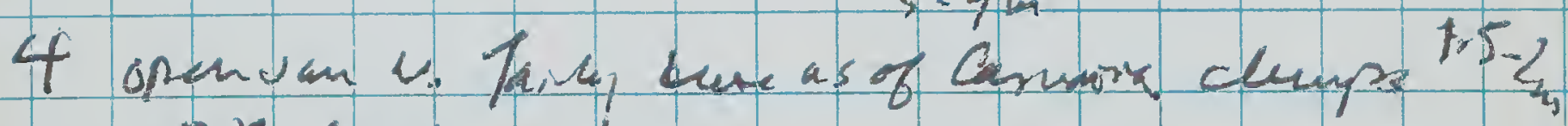
B $x$ - faced wordsur.

5 tivin denxe Cas, bruer w. Simegaps 0 6 dense Casuaring thichats $1.5 .2 \mathrm{~m}$

(Dog by ik then setion)

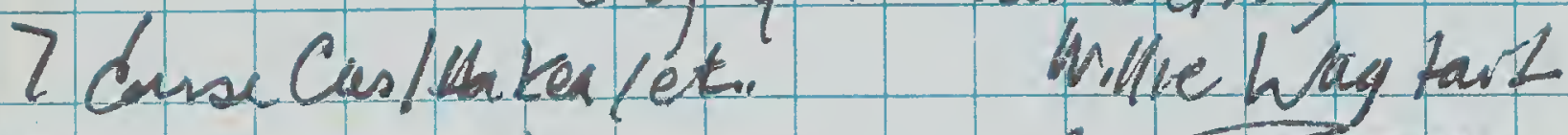
\& a ziobatuch 2 rant paver 


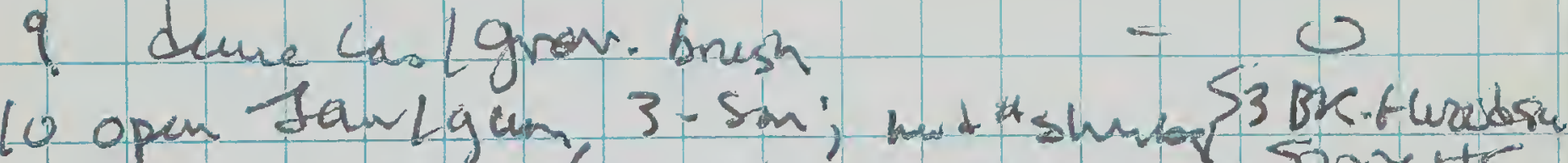

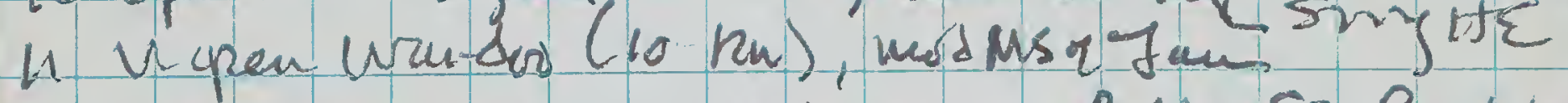
sue quensope, nod thasbs Brtte, Son Pardedre

12. Low Exe sapdurde (3-4m) u fairly deuse sumbs $\sim 6$ H.R. T-bill, 2 chrt-bill, 3 Zebva Fure, Wh-F Chat luillie wagtail

Cut into interios

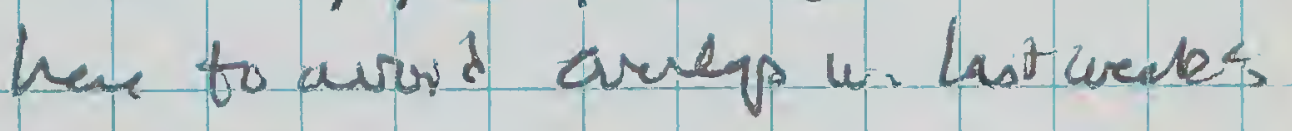

NTERTR sineys

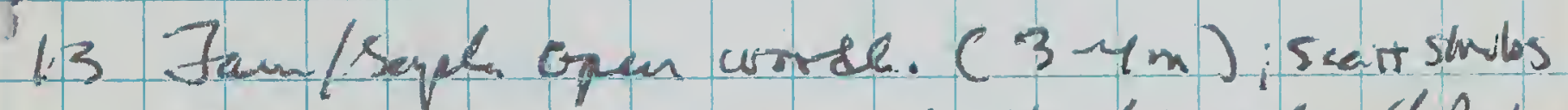
sumbites, y-v Tubill, weebsill Bidned

14 doush hew (Cas, qreviete) 1.5m 6

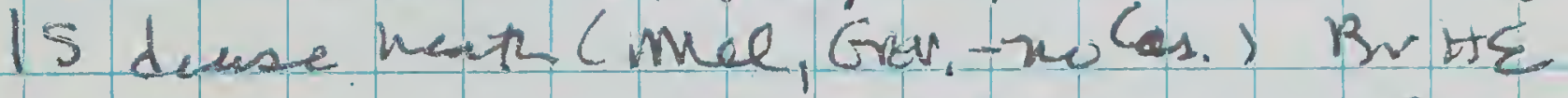

16 . Stat. malled chups to $2.5 \mathrm{~m}$

4 BK-P woodsu

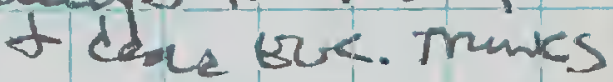

17 F deuse heaph ( Mul Acars, qrav, ete) $1,5 \mathrm{k}$ w. gat. Madee cempo to $3 \mathrm{~m}$ Y-r T-bill, o RuF wins den:

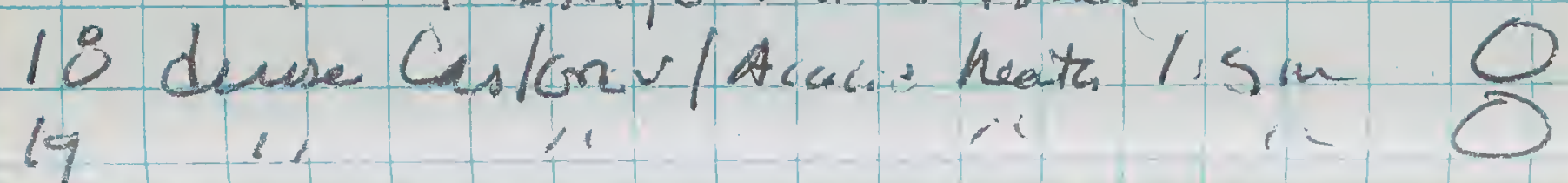

\begin{tabular}{|c|c|c|c|c|c|c|c|c|c|c|}
\hline & $B$ & $\operatorname{ces} 2$ & & & & Res & Wh & & $\pi$ & \\
\hline & & $r s i$ & & & & & & & & \\
\hline & & & & & & 11 & - & & - & $\rightarrow$ \\
\hline 70 & 2.2 & & 3 & & & & - & 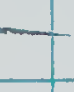 & & $\infty$ \\
\hline 21 & 12 & & 2 & & ar il & $=c c_{i l}$ & 7 & & $\Leftrightarrow$ & \\
\hline & $\pi$ & $3 \mathrm{~m}$ & & & & & & & & 0 \\
\hline
\end{tabular}

22

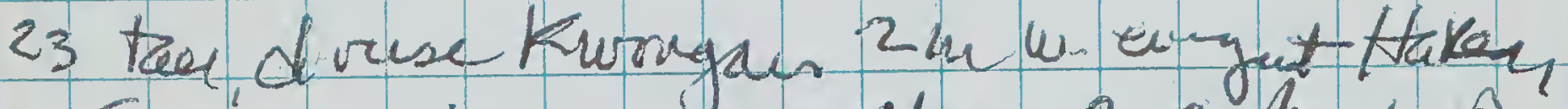

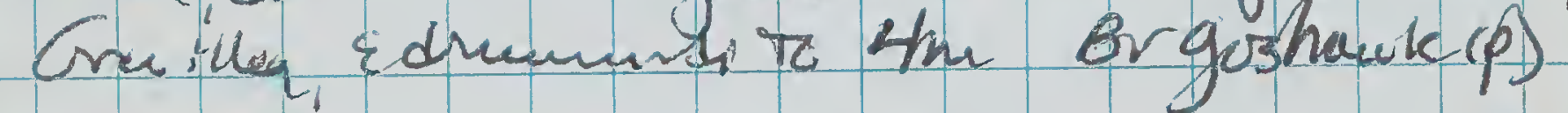

$24 \% \quad \therefore-$ C.Bronzeari

is maulee (3m) mod shwbsun is 0 2-6 Waudool Sam worde lsu ioper ms a malle, scuct shion $24-5$ Mrver

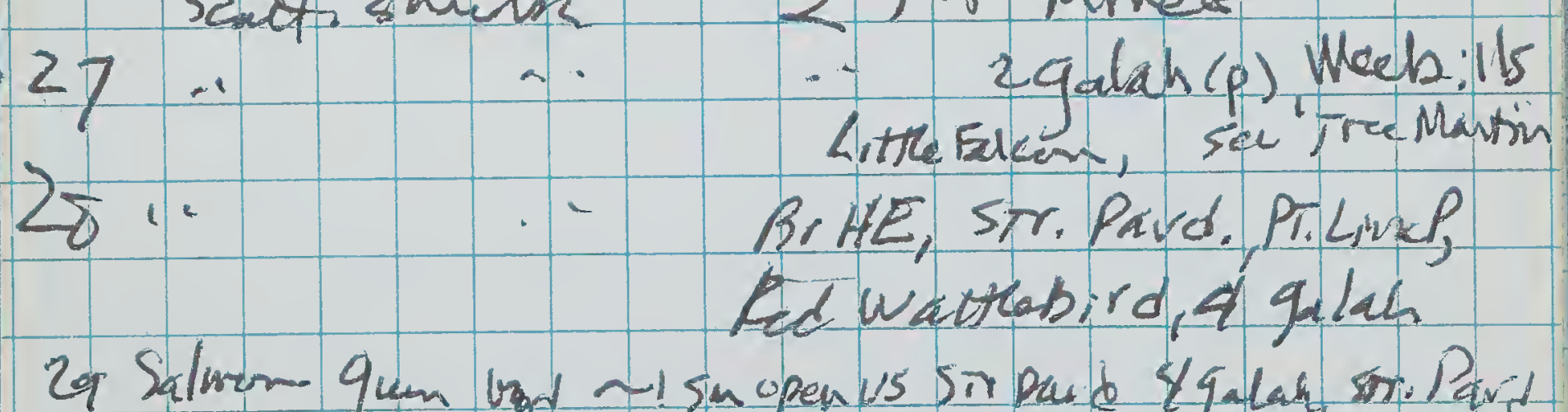

3o open sahu gom wood" " " so Pard.

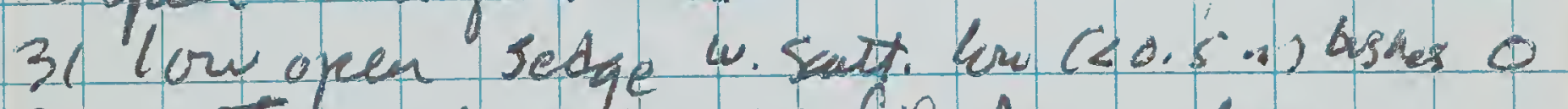
32 Tancinde $3.4 \mathrm{~m}$ W. Ging dense clumps

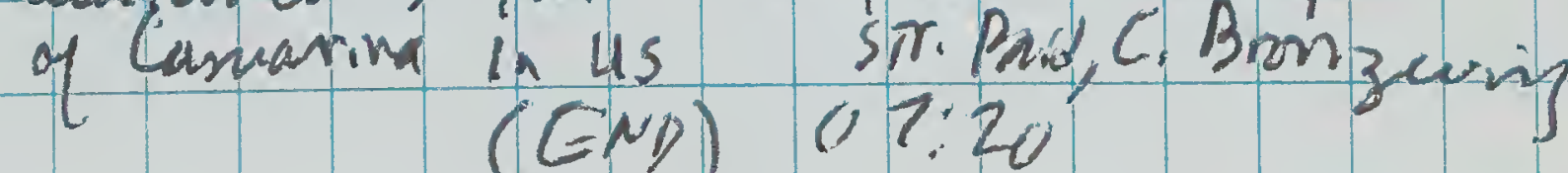


Durokoppin east - Workig earturand Fr. Ns Track $\sim 500 \mathrm{~m}$ S. of $\mathrm{N}_{2}$ burder INTERIOR

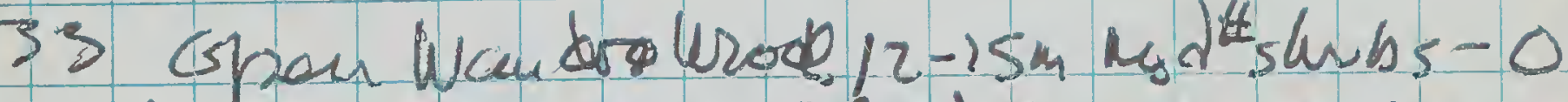

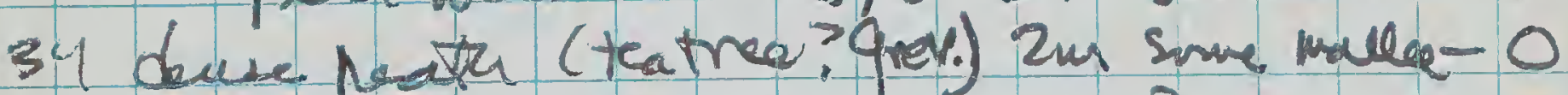
35 deure lext ( Mel, ct. $(1.5-2 m) \quad 0$ $(P-1300$ here $)$

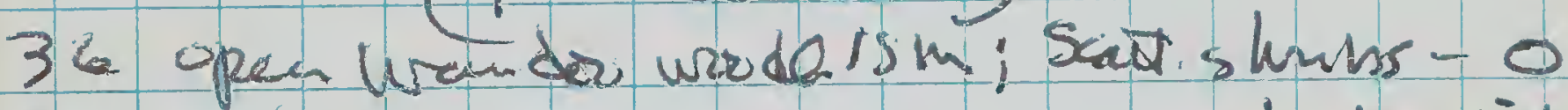
32 " "R" "Jacky Writer 38 Mooline Roo 'Seet here $3 a$ (motshubs) weelsills to a a in 0 Ql dease Las. Thichets $2 \mathrm{~m}$

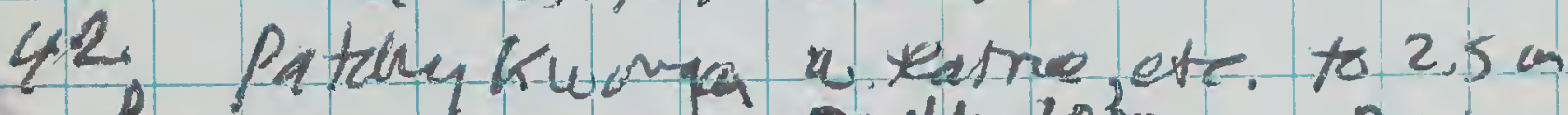
a few say waudoo 4-1000 Bvite zgale

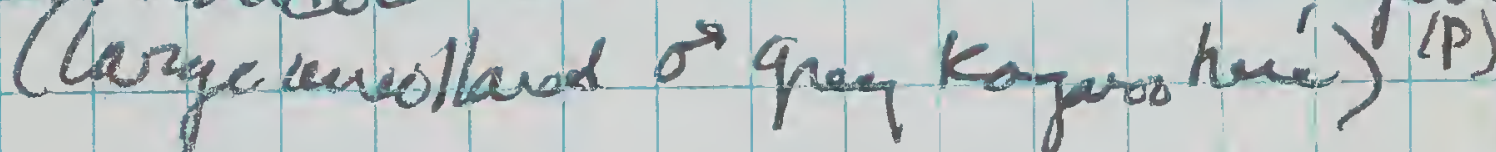

43 " 4 mad wauder 44." Dhat nuclaudod Br DE. 43 öpen las/ Jam ward (3w) u. faíly dere clemps of lous cas, keath et.

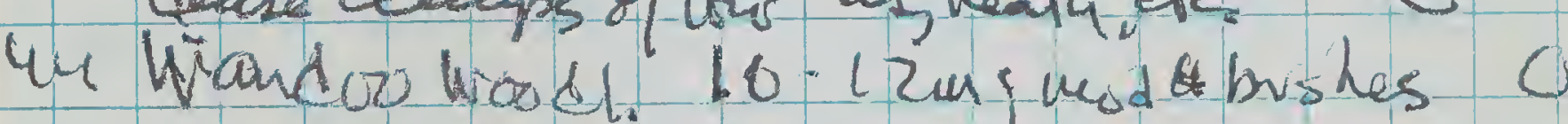
us.th ic iupen MS walle (teving) opar US - OPRRCRobin, BrHE $46 \%$ " (hut zo wallu) il il weebill (Poilseer lev) - 600 live

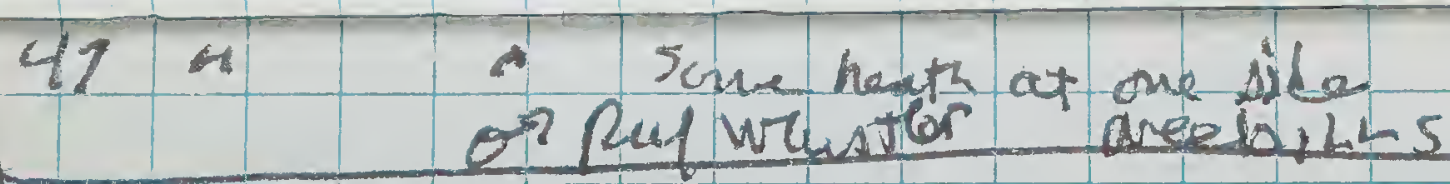

$\rightarrow$ Ruf. Wrister (unpanded) (1) hoiz suatch / an fion deg yqu pua

(2) Ho ding 0.66 a for sameperch to is levies

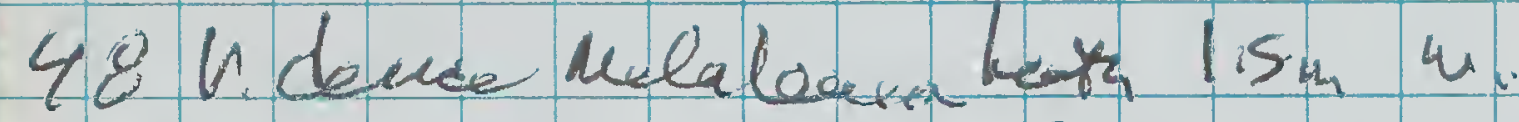

seart Walle 3-4m-Br HE

49. opm Mallee/Cas. \&.v. coase us of

'Meladenca lisun Grsmithe,

So prablie/Cas (to 4ta) a. dense mee Us - 0

si denre as tarishes $3-4 \mathrm{~m} 0$

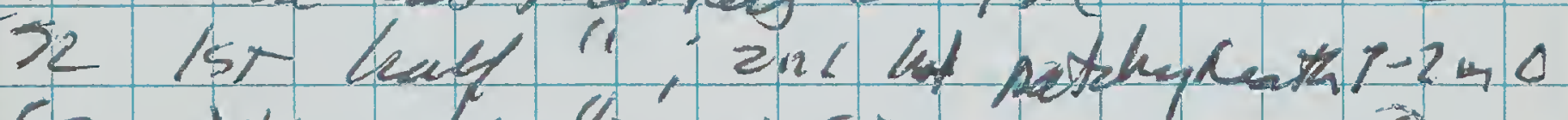
53 like las 12 * 52 


\section{Ershron 1}

Ref bunter 1

- Deruse Parvanvatidrets 2 n o

ci li cl a 0

62 " in 4 u

6.3 patchy heath wi dewe Cas Compes

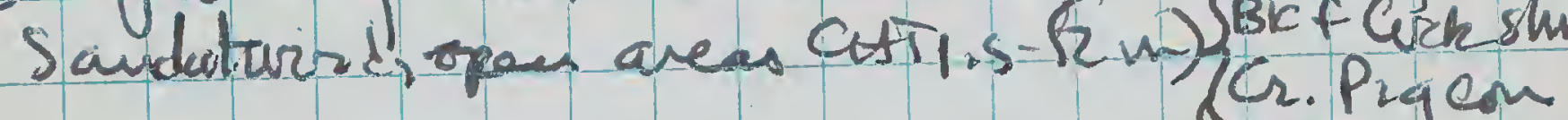

64 fainhour Cas. Thi rots 2 m-o Sno ite

c5 a i i. o

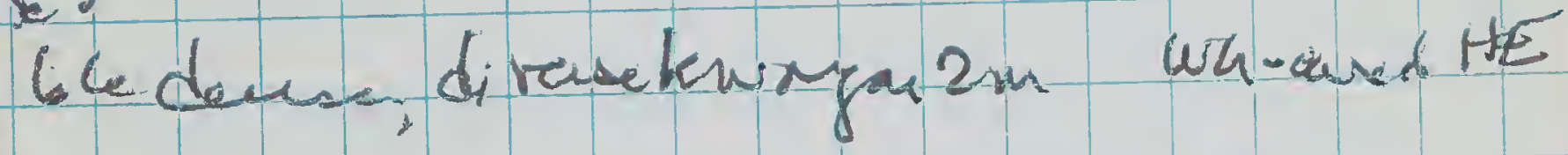
road edge som past bere

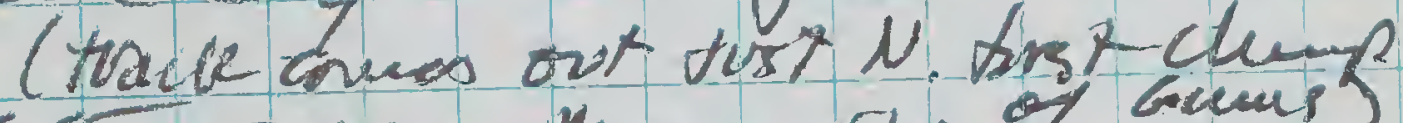

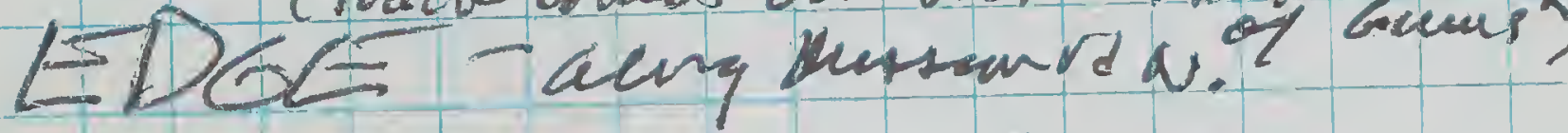

67 dense divasenong, dum by Cas, br tE 68 dene aw Rerhas $2-2.540$

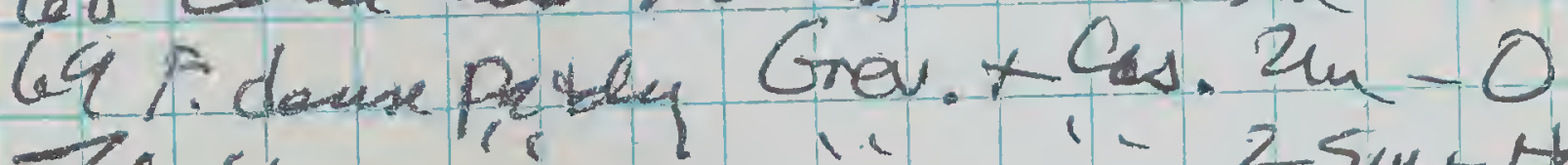
Zo a i i. '- $2 \sin \theta$ tee Z1" $\quad$ Wr sque sandeaturod 2 Magpie ( $p$ )

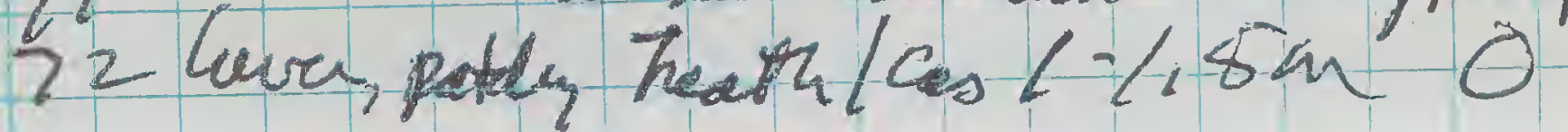

73 talten ․ $\quad \cdots \quad 2 \operatorname{sug} A_{s}$

$25<$ यद 70 :

77 low pataly heaph blasm

$78 \%$ '2m crpiqeen 29 troudenen putrigan sig tit $81 x$ u $x$ in a $82 \cdots \quad$ ".

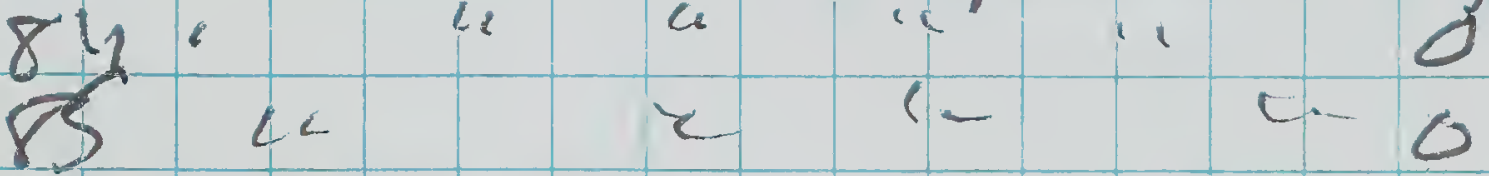

84 Wandro vade 12 - $25 m$ - denn

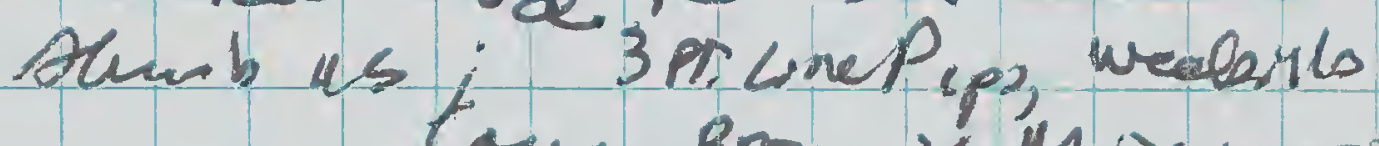
(8) Broun $x$ Miasion tem w

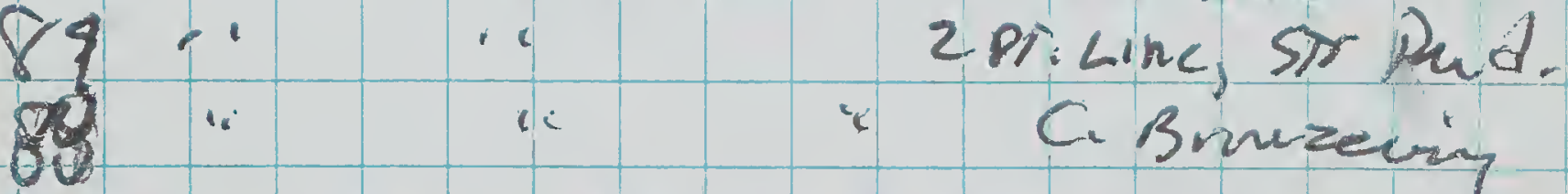

Qgoopen wander-15M; scatt shrubs =

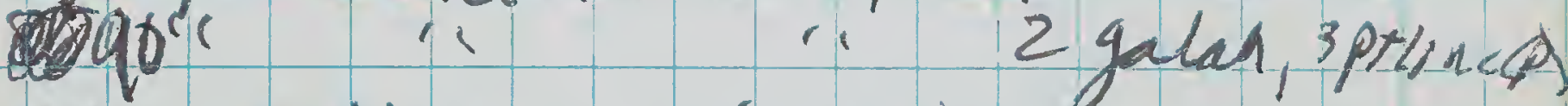
- open York word (10u, 2m) open us - 20 golah, 2 PT.L.hC, Y-T Hiver

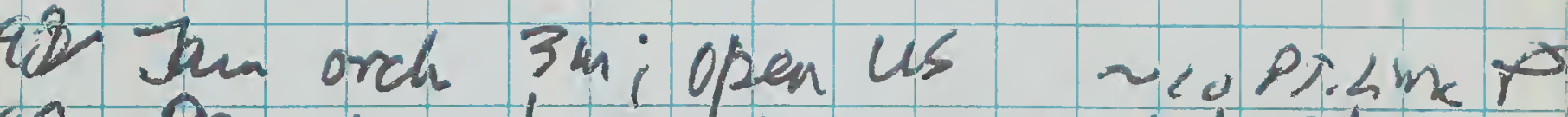

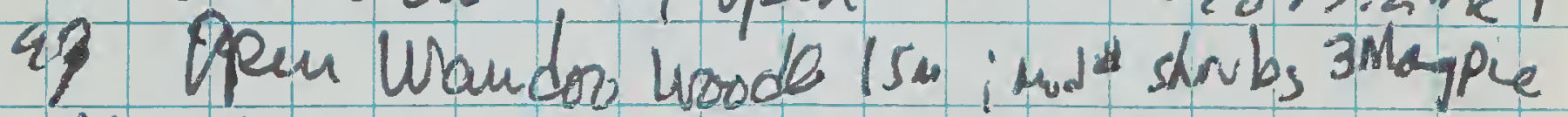
$24 \mathrm{Jan}$ ord opea us seo Y-TMines. Gr. BuTcherbird. sa PT L ma P 
95 pan fork G wride 10-1 zum w. fai By

darse Nis of Jur; open us - 0

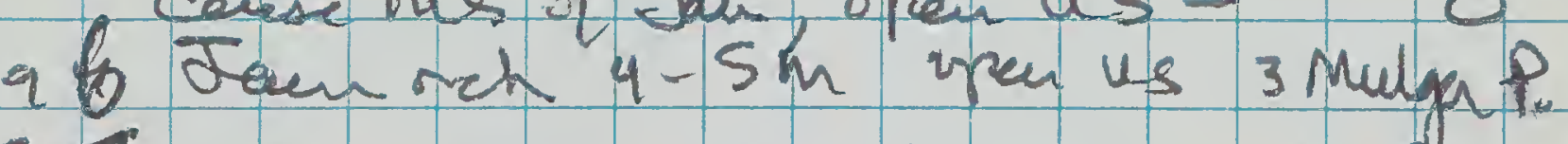

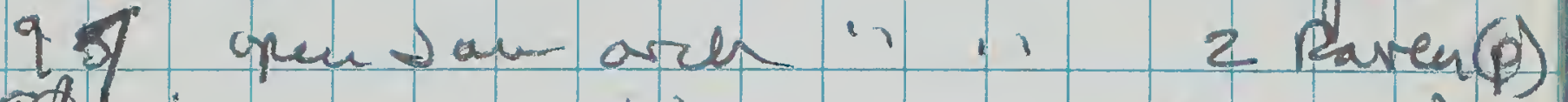
98 - . - Pr linc $P(D)$

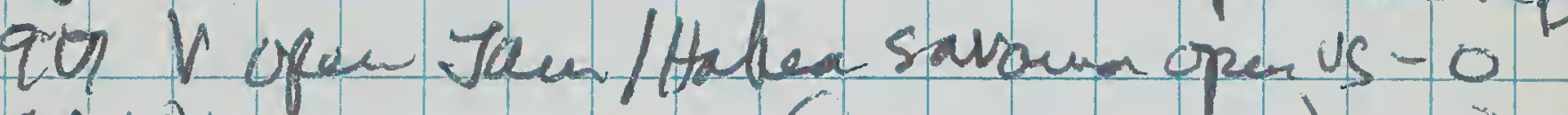
$9810) i(\cot 3-4 m) 0$ 20401 $\cos 100$ ioz opeen Hahea/quash 3.44 0 lod \%

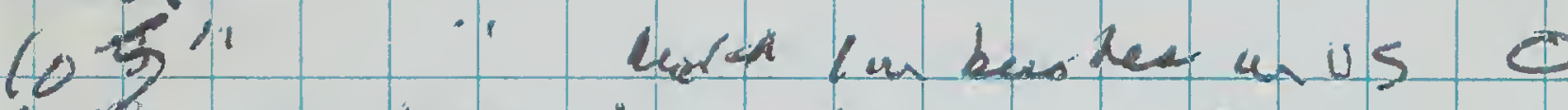
100 uper haked/Jan/Cas hod) 3.4n 4a. Mnd humens BK-furosdsw.

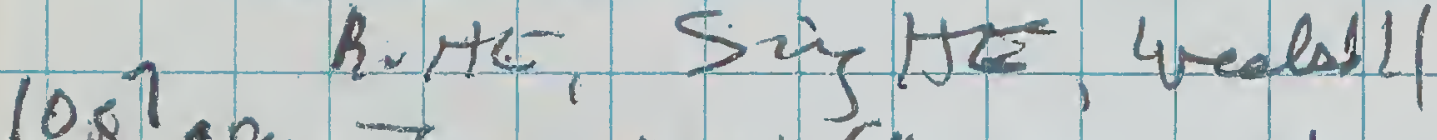

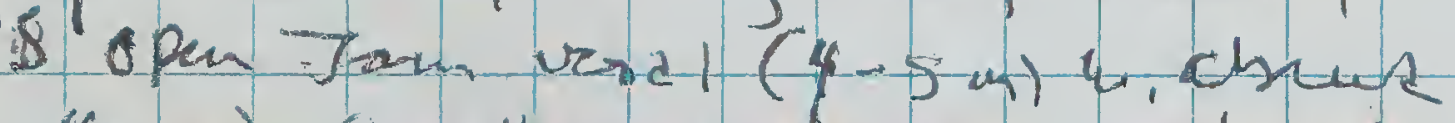

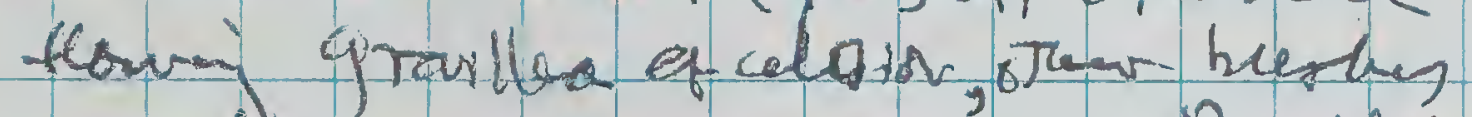
in Ms and as, Brits

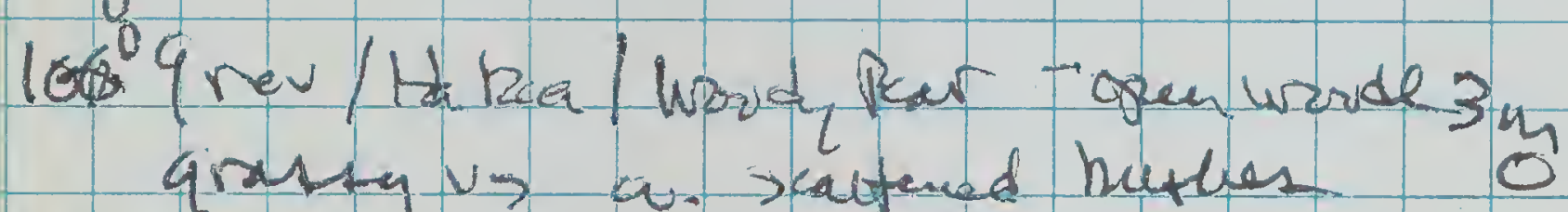

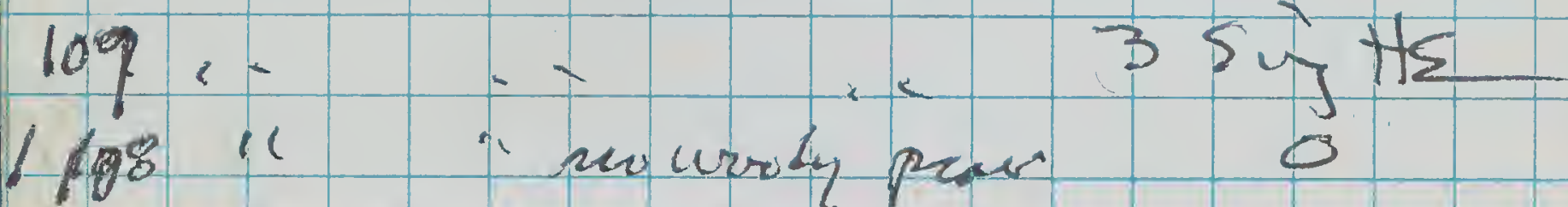

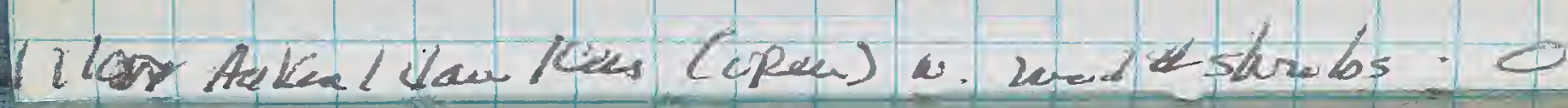

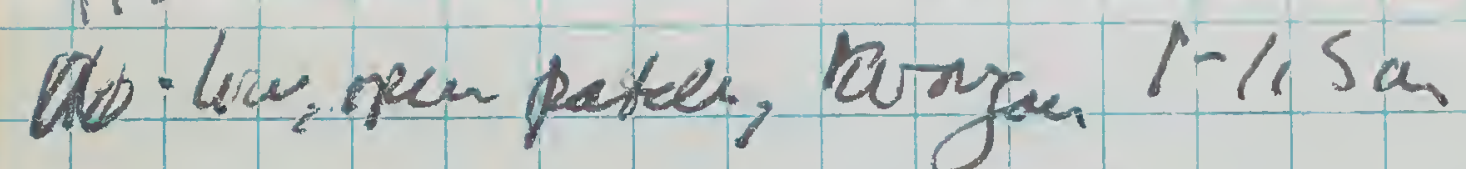
As trach her - styp

Kelenkmir 2877

kestrel 2882 \&NOV $88 \& 7,10$

1633 DJs rufi to Ceomalle Cr

1841 Mavchogetract 1853 WADJADD CRAf( m's) 

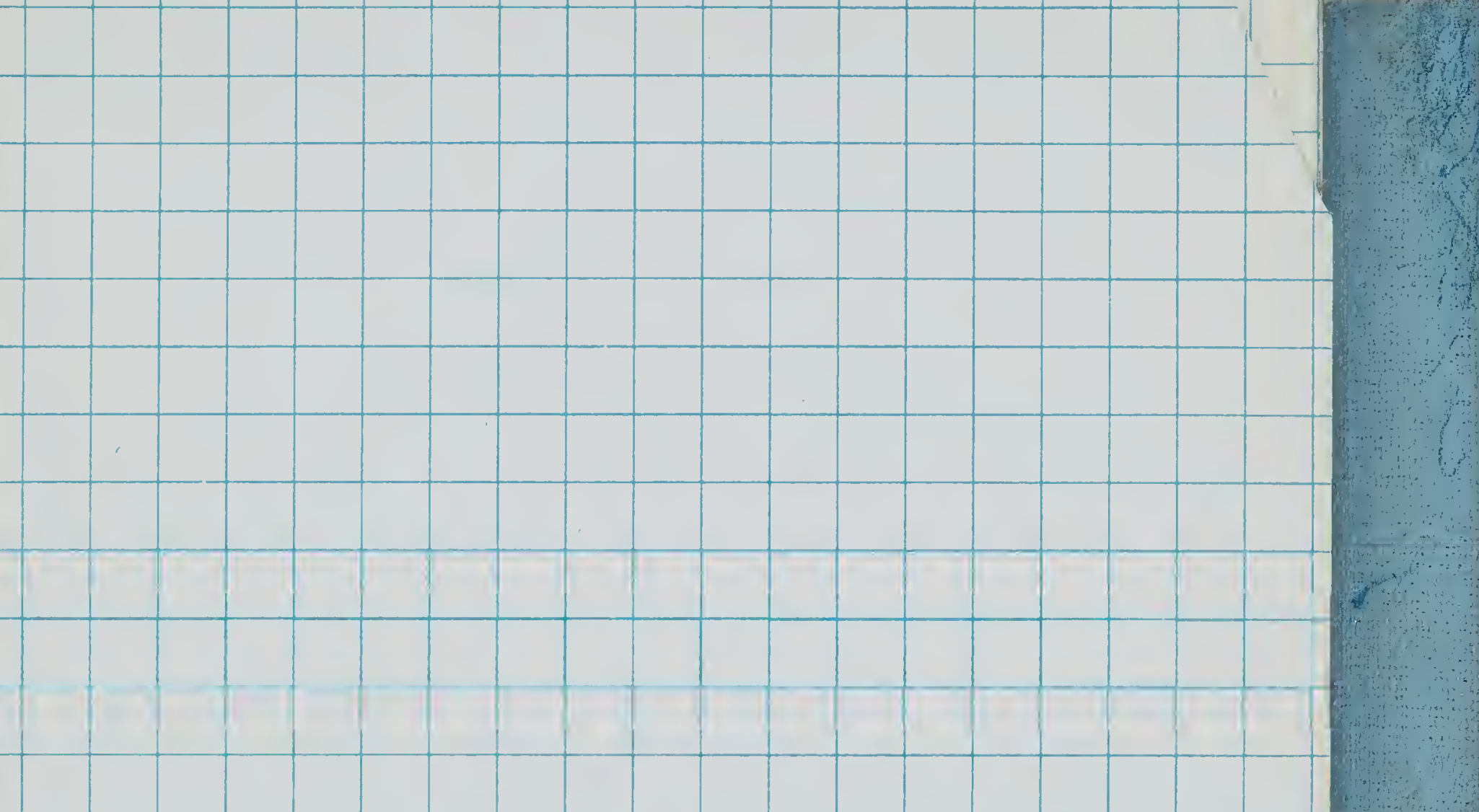

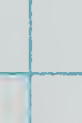
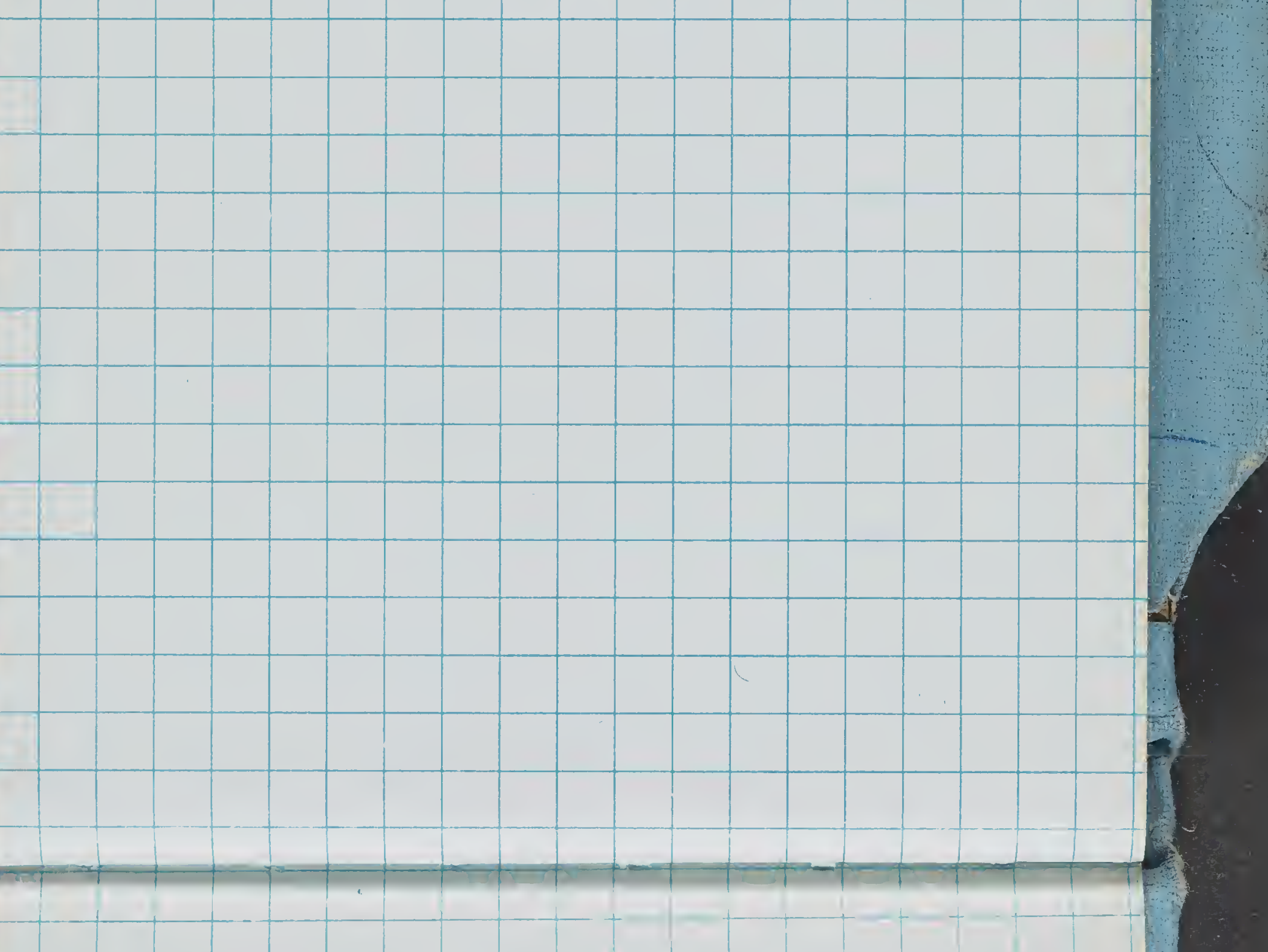

W.
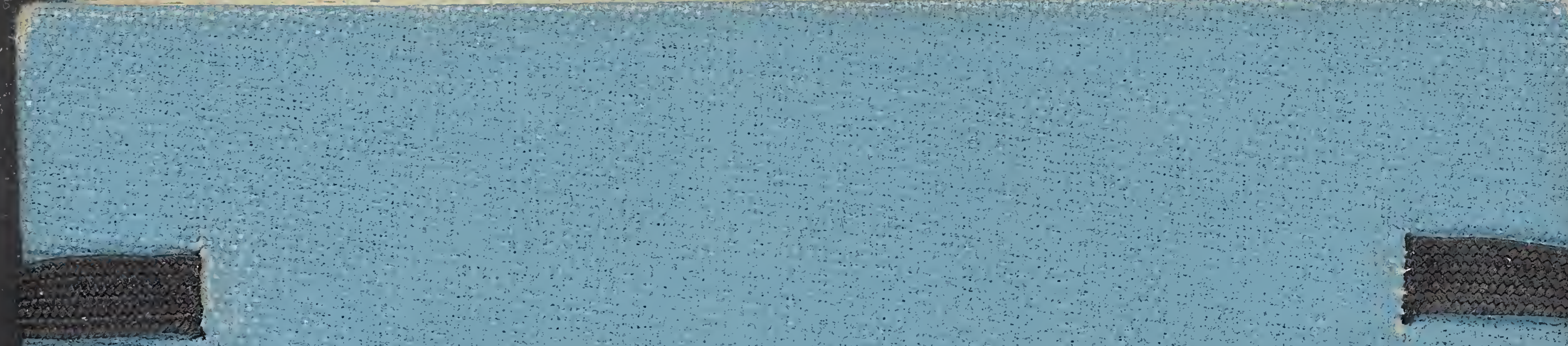

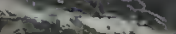
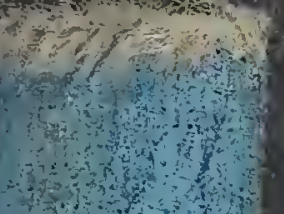

H.

al

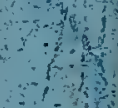

$+1$

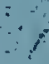

